\title{
Modeling, Identification, and Control of an Unmanned Surface Vehicle
}

\author{
by
}

\section{Christian R. Sonnenburg}

\author{
Dissertation submitted to the Faculty of the \\ Doctor of Philosophy \\ in \\ Aerospace Engineering \\ Craig A. Woolsey, Co-Chair \\ Daniel J. Stilwell, Co-Chair \\ Mazen Farhood \\ Leigh S. McCue-Weil
} Virginia Polytechnic Institute and State University

in partial fulfillment of the requirements for the degree of

December 7th, 2012

Blacksburg, Virginia

Keywords: USV, System Identification, Field Experimentation, Backstepping, Trajectory Control Copyright 2012, Christian R. Sonnenburg 


\title{
Modeling, Identification, and Control of an Unmanned Surface Vehicle
}

\section{Christian R. Sonnenburg}

\begin{abstract}
This dissertation addresses the modeling, identification, and control of an automated planing vessel. To provide motion models for trajectory generation and to enable model-based control design for trajectory tracking, several experimentally identified models are compared over a wide range of speed and planing conditions for the Virginia Tech Ribcraft Unmanned Surface Vehicle. The modeling and identification objective is to determine a model which is sufficiently rich to enable effective model-based control design and trajectory optimization, sufficiently simple to allow parameter identification, and sufficiently general to describe a variety of hull forms and actuator configurations. Beginning with a 6 degree of freedom nonlinear dynamic model, several linear steering and speed models are obtained as well as a thruster model.

The Ribcraft USV tracks trajectories generated with the selected maneuvering models by using a backstepping trajectory controller. A PD cascade trajectory control law is also developed and the performance of the two controllers is compared using aggressive trajectories. The backstepping control law compares favorably to the PD cascade controller. The backstepping control law is then further modified to account for nonlinear sternward dynamics and for a constant or slowly varying fluid flow.
\end{abstract}




\section{ACKNOWLEDGMENTS}

This dissertation is the product of the culmination of years of research and field experimentation that required the collective abilities only found in a group of skilled researchers. There are many people that guided me through the higher level decision-making and advising process as well as the more laborious experimentation and implementation efforts. Without these people I would not have been able to finish a dissertation even approaching this quality.

I must thank my advisor Dr. Craig Woolsey for single handedly accepting me into the aerospace graduate program and guiding me toward a fulfilling graduate career with Autonomous Systems and Controls Laboratory with its very capable members and research vessel, which was essential for all the experimental results found in this dissertation. His persistence and patience in advising has infused in me a work ethic I thought only few people might attain. Under his tutelage I have been guided towards interesting controls tasks. Of equal importance were his abilities to spot potential flaws in theoretical constructs as well as to know when my research had reached significant publication milestones.

Thanks goes out to my co-advisor Dr. Daniel Stilwell as well as my final two committee members Dr. Mazen Farhood and Dr. Leigh McCue-Weil. Without Dr. Stilwell and his lab's members and Ribcraft USV I would not be able to identify models and formulate control algorithms for a planning USV. His inquisitive 
mind has helped me think critically about many issues that surfaced through my research while his grounded advice in the proper ways to present my findings has helped put my research in the most favorable light. My committee members' advice in matters of dissertation topic selection as well as vessel maneuvering dynamics has immensely added to the richness and applicability of the content of my dissertation.

Finally, I need to thank Aditya Gadre and Shu Du for their unlimited tolerance to accompany me in my often long days spent collecting data and debugging control laws. Though they will underplay their significance in my accomplishments they played an integral role. Furthermore, Aditya's sage like knowledge of $\mathrm{C}++$ and matters pertaining to device and software interaction accelerated the implementation of my nonlinear control laws. 


\section{TABLE OF CONTENTS}

Acknowledgments

List of Figures viii

List of Tables $\quad$ xi

1 Introduction $\quad 1$

2 Background $\quad 15$

2.1 Modeling . . . . . . . . . . . . . . . . . . . . 15

2.2 Parameter Identification . . . . . . . . . . . . . . . . . . . . . 17

2.3 Trajectory Tracking . . . . . . . . . . . . . . . . . . 19

3 Ribcraft USV $\quad 22$

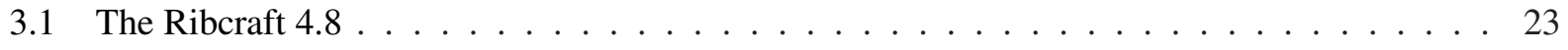


3.2 The Autonomy Hardware . . . . . . . . . . . . . . . . . . . 23

3.2 .1 Input Control Hardware $\ldots \ldots \ldots \ldots \ldots$

3.2 .2 Sensor Hardware . . . . . . . . . . . . . . . . . . . . . 27

3.3 The Autonomy Software . . . . . . . . . . . . . . . . . . 32

4 USV Dynamic Modeling $\quad 38$

4.1 Kinematics . . . . . . . . . . . . . . . . . . . . . . 39

4.2 Nonlinear 6 DOF Dynamics $\ldots \ldots \ldots \ldots \ldots \ldots$

4.3 Planar Kinematics . . . . . . . . . . . . . . . . . . . . . . . . . . . . 49

4.4 Planar Nonlinear 3 DOF Dynamics . . . . . . . . . . . . . . . . . . 50

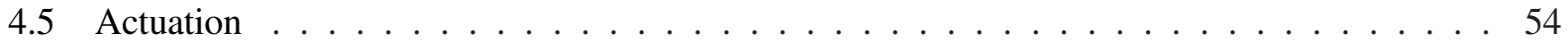

4.6 Simplified Vessel Dynamics _. . . . . . . . . . . . . . . . . 55

$4.6 .1 \quad$ Speed Dynamics $\ldots \ldots \ldots \ldots \ldots \ldots$

4.6.1.1 Quadratic Drag Speed Model . . . . . . . . . . . . . 56

4.6.1.2 Linear Drag Speed Model . . . . . . . . . . . . . . . . . 56

4.6 .2 Steering Dynamics $\ldots \ldots \ldots \ldots \ldots \ldots$

4.6.2.1 Linear Steering Dynamics . . . . . . . . . . . . . . . . . . 58

4.6.2.2 Nomoto's Steering Models _. . . . . . . . . . . . . 58

4.6.2.3 Considering Sternward Steering Behavior _ . . . . . . . . . . . . 59

5 Model Identification $\quad 61$

5.1 Identifying Steady-State Input/Output Relationships ～. . . . . . . . . . . . . . . 62

5.2 Identifying Dynamic Parameters $\ldots \ldots \ldots \ldots \ldots \ldots$

5.2 .1 Identifying Thruster Parameters . . . . . . . . . . . . . . . 64

5.2.2 Identifying Linear State Transient Dynamics _ . . . . . . . . . . . . . . 66

5.3 Model Identification Results $\ldots \ldots \ldots 6$

5.3.1 Steady-State Input/Output Relationships . . . . . . . . . . . . . . . . . . 67

$5.3 .2 \quad$ Actuator Nonlinearities . . . . . . . . . . . . . . . . . 68 
5.3 .3 Thruster Model . . . . . . . . . . . . . . . . . . . . . 71

5.3.4 Speed and Steering Dynamic Parameters _. . . . . . . . . . . . . 72

5.3.5 Special Considerations: Sternward Steering Bifurcation Behavior . . . . . . . . . . 84

6 Trajectory Tracking $\quad 97$

6.1 Problem Formulation . . . . . . . . . . . . . . . . . . . . . . 98

6.2 A Cascaded PD Control Approach _ . . . . . . . . . . . . . . . . . . 101

6.3 A Backstepping Control Approach . . . . . . . . . . . . . . . . . . 106

6.3.1 Incorporating Model Parameter Scheduling in Backstepping Control . . . . . . . . . 114

6.3.1.1 Discrepancies in Parameters . . . . . . . . . . . . . . 115

6.3.1.2 Discontinuities in the Lyapunov Function . . . . . . . . . . . . . 118

6.3.2 Including Sternward Bifurcation Dynamics _ . . . . . . . . . . . . . . . 124

6.3.3 Including Steady and Slowly Varying Flow . . . . . . . . . . . . . . 126

6.4 Control Results . . . . . . . . . . . . . . . . . . . . . . . . 129

6.4.1 Forward Motion Experimental Results . . . . . . . . . . . . . . . . . 130

6.4.2 Sternward Motion Experimental Results . . . . . . . . . . . . . . . . . . . 134

6.4.3 Control in Flow Simulation Results . . . . . . . . . . . . . . . . . 135

7 Conclusions $\quad 140$

A Identifiability Calculations $\quad 156$

A.1 Nomoto's Model and Linearized Speed Model . . . . . . . . . . . . . . . . . 157

A.2 Nomoto's Model with Sideslip . . . . . . . . . . . . . . . . 158

A.3 Planar Steering Model _ . . . . . . . . . . . . . . . . . . 160 


\section{LIST OF FIGURES}

1.1 Maritime Robotics/NTNU USVs (Permission granted by Maritime Robotics) . . . . . . . 3

1.2 ISSIA-CNR USVs (Permission granted by ISSIA-CNR) $\ldots \ldots \ldots \ldots$

1.3 USVs developed at Instituto Superior Téchic (Permission granted by IST) $\ldots \ldots \ldots$

1.4 USVs developed at Instituto Superior de Engenharia do Porto (Permission granted by ISEP) 6

1.5 USVs developed at the Massachusetts Institute of Technology (Permission granted by MIT) . 7

1.6 The University of Plymouth's Springer USV (Permission granted by UP) $\ldots \ldots$

1.7 Israeli military USVs $\ldots \ldots \ldots \ldots \ldots \ldots$

1.8 Swedish military mine sweepers and patrol USVs (Permission granted by Kockums) . . . 10

1.9 Blackfish USV (Permission granted by QinetiQ North America) . . . . . . . . . . . . 11

1.10 American USVs . . . . . . . . . . . . . . . . . . . . . . . 12

3.1 The Vicor, relay board, and fuse board in the Ribcraft USV's electronics box . . . . . . . . . 24

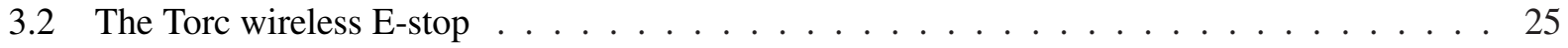


3.3 The op amp board and motor controller $\ldots \ldots \ldots \ldots \ldots$

3.4 The Raymarine position sensor for steering feedback . . . . . . . . . . . . . . 27

3.5 The emergency stop and electronic throttle lever . . . . . . . . . . . . . . . 28

3.6 The digital potentiometer converting serial commands into analog voltage . . . . . . . . 29

3.7 The Smart Actuator and Analog Control Interface to control throttle . . . . . . . . . . 30

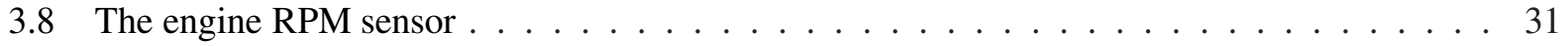

3.9 The DGPS, gimbal, laser line scanner, and AHRS . . . . . . . . . . . . . . . 32

3.10 The fully autonomous Ribcraft USV . . . . . . . . . . . . . . . . . 37

4.1 Body fixed 6 DOF reference frame $\ldots \ldots \ldots \ldots$. . . . . . . . . . . . . 40

4.2 Schematic depiction of a USV . . . . . . . . . . . . . . . . . . . 49

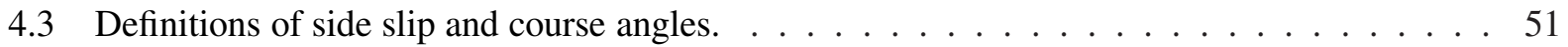

5.1 Steady state relationships for the Ribcraft USV . . . . . . . . . . . . . . . 69

5.2 Steady state speed fits $\ldots \ldots \ldots \ldots \ldots \ldots \ldots$

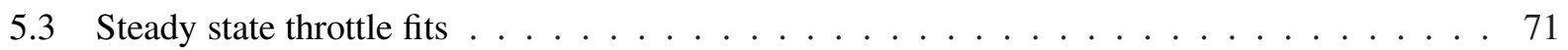

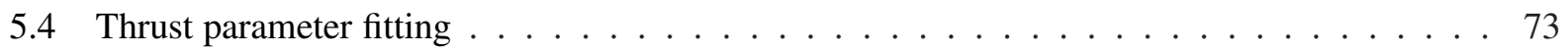

5.5 Estimated thrust and drag for the nonlinear speed model in the displacement region $\ldots . .74$

5.6 Estimated thrust and drag for the nonlinear speed model in the planing region $\ldots \ldots 75$

5.7 Actual and estimated forward acceleration for a range of forward speeds $\ldots \ldots \ldots$

5.8 Actual and estimated turn acceleration for a range of forward speeds . . . . . . . . . 77

5.9 Actual vs. estimated sideslip angular rate for a range of forward speeds $\ldots \ldots$

5.10 Measured forward speed vs. simulated forward speed for a range of forward speeds $\ldots . .80$

5.11 Actual and simulated turn rate time histories for a range of forward speeds $\ldots \ldots \ldots 1$

5.12 Actual vs. simulated sideslip angle time histories for a range of forward speeds $\ldots$. . . . 82

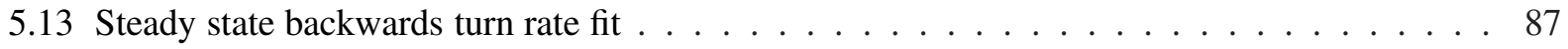

5.14 Thrust fit for sternward motion $\ldots \ldots \ldots \ldots \ldots$

5.15 Estimated thrust and drag for the linear and nonlinear speed models for sternward motion . . 89 
5.16 Actual and estimated turn acceleration for the two stable and one unstable turn rate regions . 90

5.17 Actual vs. estimated sideslip angular rate for the two stable and one unstable turn rate regions 91

5.18 Actual and simulated turn rate time histories for the two stable and one unstable turn rate

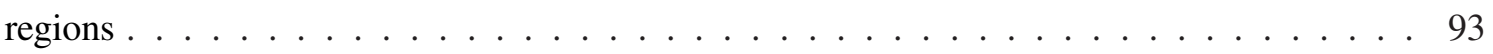

5.19 Actual vs. simulated sideslip angle time histories for the two stable and one unstable turn

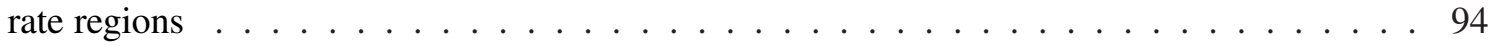

6.1 Notation and formulation of cross track and along track error. . . . . . . . . . . . 100

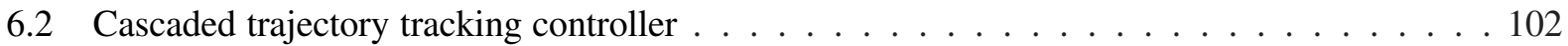

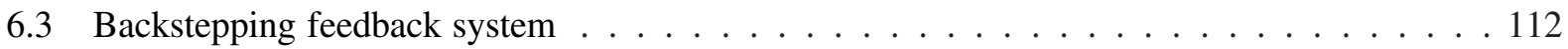

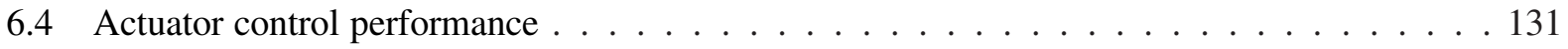

6.5 Heading and speed tracking performance . . . . . . . . . . . . . . 132

6.6 Trajectory tracking control for constant propeller speed and a sinusoid in rudder . . . . . . 133

6.7 Trajectory tracking control for sinusoids (of differing frequency) in propeller speed and rudder 135

6.8 Trajectory tracking control for constant propeller speed and a sinusoid in thrust angle in

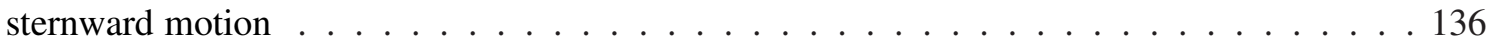

6.9 Actual actuator inputs of propeller speed and rudder position used in the trajectory tracking

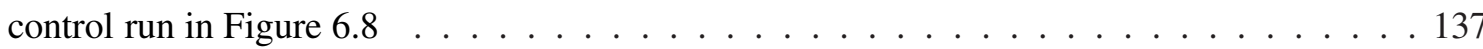

6.10 Trajectory tracking control for constant propeller speed and a sinusoid in thrust angle with the generated reference trajectory and actual vessel both being convected with flow . . . . 138

6.11 Trajectory tracking control for constant propeller speed and a sinusoid in thrust angle with the reference trajectory generated assuming no flow with the actual vessel in flow . . . . . . 139 


\section{LIST OF TABLES}

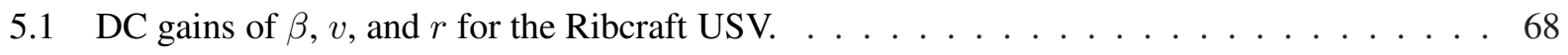

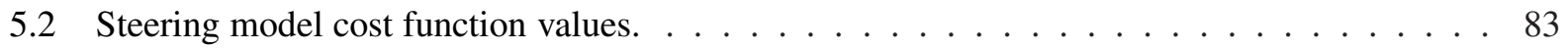

5.3 Linear steering model parameter values (using linear fit of steady-state gains). . . . . . . . 84

5.4 Nomoto-with-sideslip steering model parameter values. . . . . . . . . . . . . 85

5.5 Nomoto steering model parameter values. . . . . . . . . . . . . . 85

5.6 First order linear speed model parameter values. . . . . . . . . . . . . . . 86

5.7 Sternward steering model cost function values. . . . . . . . . . . . . . . 92

5.8 Linear steering model parameter values for sternward motion . . . . . . . . . . . 95

5.9 Nomoto-with-sideslip steering model parameter values for sternward motion . . . . . . . . 95

5.10 Nomoto steering model parameter values for sternward motion . . . . . . . . . . . . 95

5.11 Speed and steering model parameter values. (Parameter units suppressed.) . . . . . . . . . 96

6.1 Control Gains for the PD control system for the Ribcraft USV . . . . . . . . . . . . 106 
6.2 Ribcraft USV Closed-Loop Linearized PD Control System Eigenvalues ～. . . . . . . . 106

6.3 Ribcraft USV Closed-Loop Linearized Backstepping Eigenvalues ～. . . . . . . . . . . 114

6.4 Ribcraft USV Linearized Backstepping Control Gains . . . . . . . . . . . . . . . . . . . . 114 


\section{CHAPTER}

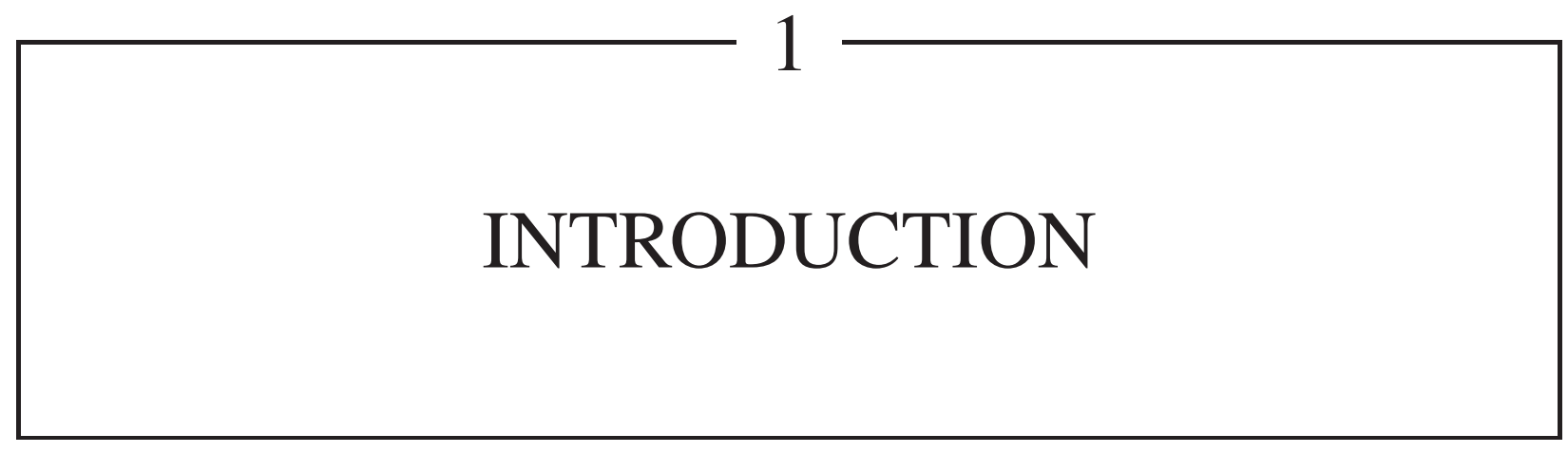

Riverine operations pose extraordinary challenges for the U.S. Navy. The helmsman of a riverine vessel must quickly and safely execute maneuvers to traverse unknown environments. Increasing the helmsman's prior knowledge of his surroundings allows for maneuvers to be executed at higher speeds, decreasing the time necessary for sortie completion and giving an enemy combatant less time to react and retaliate.

The roles these servicemen play are invaluable, but every opportunity must be taken to mitigate casualties and loss of life. The best way to increase safety is to remove the servicemen from their most dangerous tasks altogether by automating the vessels they operate. For cases where the presence of servicemen is still needed, automated vessels can provide advance reconnaissance. Autonomous vessels must reliably perform aggressive maneuvers to successfully replace highly skilled human operators, requiring development of advanced control algorithms. One facet of riverine robotics research [Thomsen et al., 2007] is focused on developing methods to make the most dangerous tasks autonomous. 
Many research, military, and commercial organizations are forging ahead with unmanned surface vehicle (USV) development. Often manned vessels are converted to autonomous operation, though more and more USVs are being designed for autonomous operation from their inception. USVs are outfitted with global positioning system (GPS) receivers and attitude and heading reference system (AHRS) sensors for closedloop waypoint following and trajectory tracking. Other ancillary sensors like doppler velocity logs (DVL) measure fluid flow allowing for even tighter control to waypoints and trajectories. Vessels on reconnaissance missions may be equipped with laser line scanners and sound navigation and ranging (SONAR) equipment to sense above surface and below surface obstacles respectively.

Research institutions develop USVs as test beds for various sensors and control algorithms. To expedite research progress most of these USVs are converted manned vessels that use collections of off the shelf components. Converted manned vessels have the added benefit of accommodation for occupants, which can be used in the preliminary stages of control and sensor development. Once the performance of experimental sensors and/or control logic have been validated, the results can be transitioned to commercial and military uses.

The Norwegian University of Science and Technology in conjunction with Maritime Robotics has three types of research vessels at their disposal. The aluminum planing monohull Kaasboll USV shown in Figure 1.1(a) measures $5.75 \mathrm{~m}$ in length and is equipped with a $50 \mathrm{hp}$ Evinrude outboard E-tec motor propelling the vessel to up to $20 \mathrm{kts}$. Fully actuated throttle and rudder are controlled using proportional controllers. Vessel position, heading, velocity, and turn rate are all output from a Seapath 20 navigation system. An onboard computer directly interfaces with MATLAB/Simulink software. The Viknes USV pictured in Figure 1.1(b) provides an all weather capability with its encapsulated cockpit. The vessel is an $8.3 \mathrm{~m}$ long semi-displacement vessel with an inboard Yanmar $184 \mathrm{hp}$ motor propelling the vessel to $20 \mathrm{kts}$. The Viknes USV and Kaasboll USV contain the same sensor and computer suite. The $6.6 \mathrm{~m}$ long planning hull Mariner USV shown in Figure 1.1(c) seeks to bridge the gap between research and application. The Mariner USV uses the same software and hardware as its predecessors, though, with a top speed of $38 \mathrm{kts}$ due to its $150 \mathrm{hp}$ Evinrude E-tec, the vessel clearly has a performance edge. The primary research purposes for these vessels involve a variety of waypoint control, target tracking, path following, and trajectory tracking 
algorithms [Breivik, 2010].

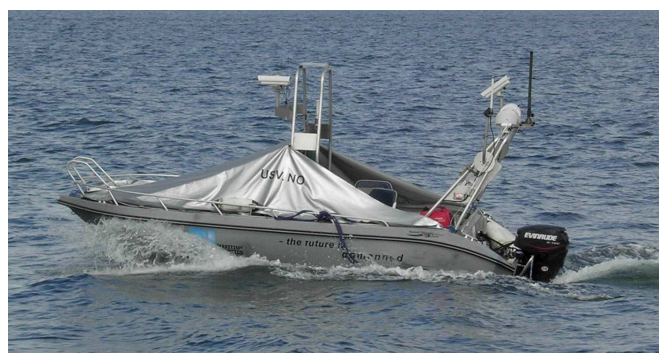

(a) Kaasboll USV

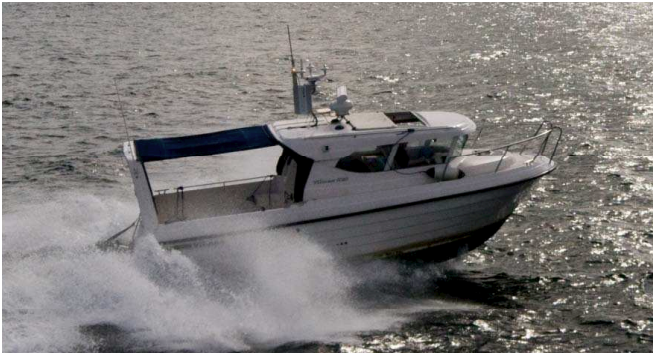

(b) Viknes USV

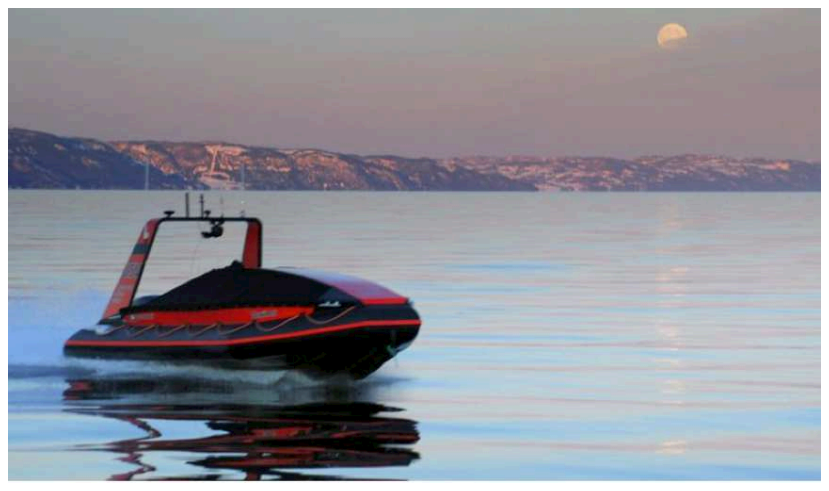

(c) Mariner USV

Figure 1.1: Maritime Robotics/NTNU USVs (Permission granted by Maritime Robotics)

The Institute of Intelligent Systems for Automation (ISSIA), part of the National Research Council (CNR) located in Italy, uses their two USVs to test a variety system identification [Caccia et al., 2006, Caccia et al., 2008b, Mišković et al., 2011], control [Bibuli et al., 2007, Bibuli et al., 2008, Bibuli et al., 2009], and path planning algorithms [Bruzzone et al., 2011]. The Aluminum Autonomous Navigator for Intelligent Sampling (ALANIS) shown in Figure 1.2(a) collects water samples and monitors the sea floor at sites deemed interesting by its control logic [Caccia et al., 2009]. This vehicle, measuring $4.5 \mathrm{~m}$ in length, is equipped with a $50 \mathrm{hp} \mathrm{Honda} \mathrm{outboard} \mathrm{motor.} \mathrm{Rudder} \mathrm{and} \mathrm{throttle} \mathrm{actuation} \mathrm{is} \mathrm{accomplished} \mathrm{through} \mathrm{the} \mathrm{use} \mathrm{of} \mathrm{two}$ brushless motors coupled with simple proportional control. ALANIS uses a Garmin 152 GPS for localization, a Navicontrol smart compass SC1G measuring yaw, and Applied Geomechanics IRIS MD900-TW Wide-Angle clinometer measuring pitch and roll. An automatic winch deploys and recovers the scientific 
instrumentation necessary for taking water samples. The Charlie USV shown in Figure 1.2(b) is a purpose built catamaran USV [Caccia et al., 2007, Caccia et al., 2008a]. Two DC motors powered by four lead acid batteries replenished by solar cells drive the $2.4 \mathrm{~m}$ vessel to a top speed of $2 \mathrm{~m} / \mathrm{s}$. The Charlie USV has a GPS Ashtech GG24C for localization, a Kvh Azimuth Gyrotrac for yaw information, and an Applied Geomechanics IRIS MD900-TW Wide-Angle clinometer measuring pitch and roll. The vessel is also equipped with an anemometer, altimeter echo sounder, and side scan sonar. $\mathrm{C}++$ control code runs on a single board computer with a Linux operating system (OS). Research on the Charlie USV includes modeling and identification [Mišković et al., 2011], regulation tasks like speed and yaw control, line following [Bibuli et al., 2008], path following [Bibuli et al., 2007, Bibuli et al., 2009], and path planning algorithms for tasks like mine hunting [Bruzzone et al., 2011].

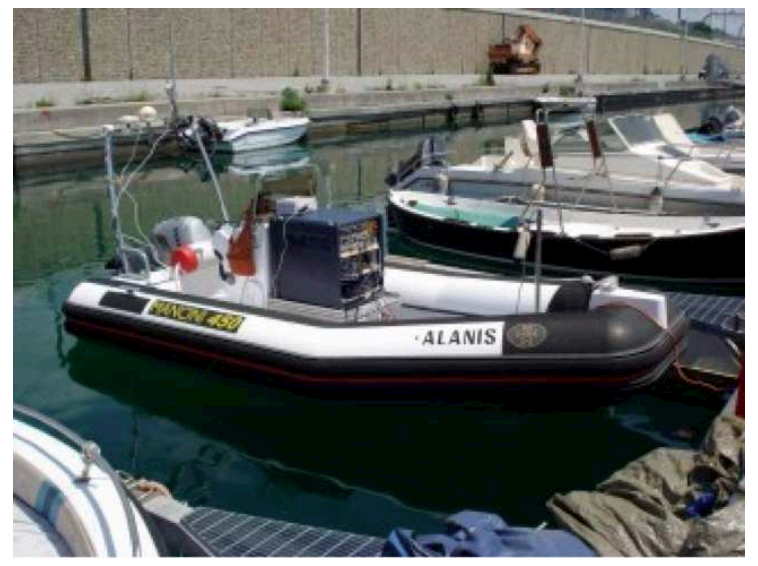

(a) ALANIS USV

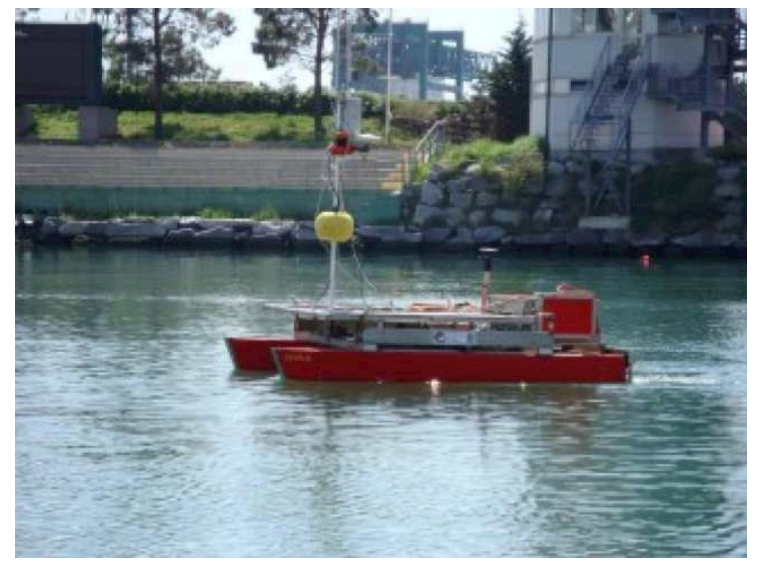

(b) Charlie USV

Figure 1.2: ISSIA-CNR USVs (Permission granted by ISSIA-CNR)

The Caravela USV and Delfim USV, developed at the Instituto Superior Téchic, and the ROAZ USV and ROAZ 2 USV, developed at the Instituto Superior de Engenharia do Porto, are Portuguese USVs primarily used to research automation of oceanographic data collection. The Caravela USV, still in the initial development phase, will be $7 \mathrm{~m}$ long, $2 \mathrm{~m}$ wide, and $6 \mathrm{~m}$ tall. This vehicle, pictured in Figure 1.3(a), has an anticipated range of $700 \mathrm{~km}$ achieved by using its 2 electric motors to propel itself to $4 \mathrm{kts}$. The Delfim USV is a $3.5 \mathrm{~m}$ long autonomous catamaran, shown in Figure 1.3(b), developed as a testbed to research 
close interaction capabilities with underwater autonomous vehicles. Using its two electric motors, a DGPS, attitude sensor, and echosounder in conjunction with mission specific navigation, guidance, and control algorithms the Delfim USV acoustically communicates with underwater assets [Alves et al., 2006]. The ROAZ USVs(Figure 1.4(a)) and ROAZ 2 (Figure 1.4(b)) [Martins et al., 2007b] are riverine and oceangoing autonomous electric powered catamarans used to test unmanned oceanographic data collecting algorithms and sensors as well as control laws focusing on coordinating multiple robots [Martins et al., 2007a]. Both ROAZ USVs use side-scan sonar and conductivity, temperature, depths (CTD) sensors for bathymetry and underwater vehicle docking. Additionally equipped with a thermographic camera and surface radar, the ROAZ 2's mission capability expands to include harbor patrol and obstacle avoidance [Almeida et al., 2009].

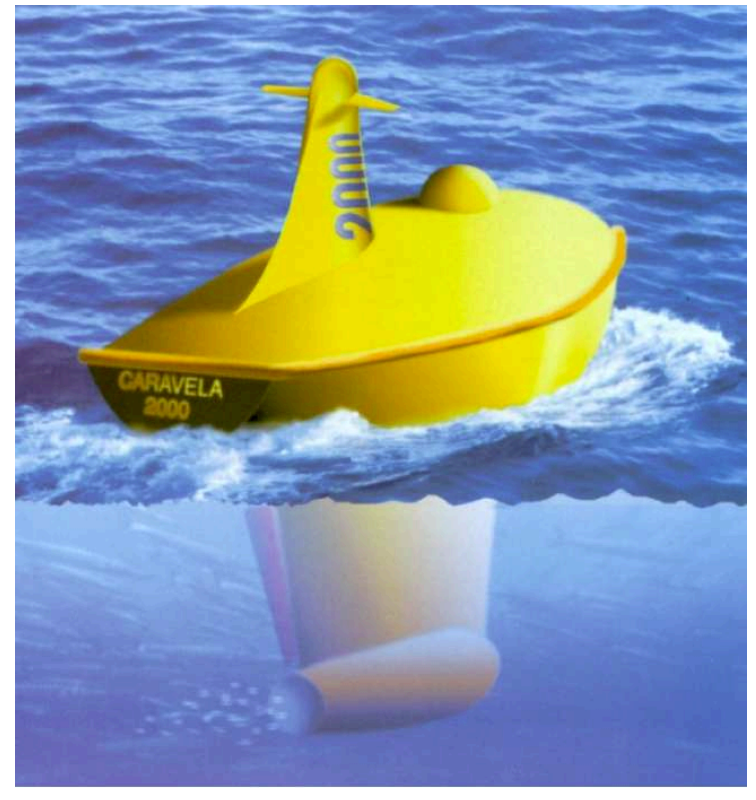

(a) Caravela USV

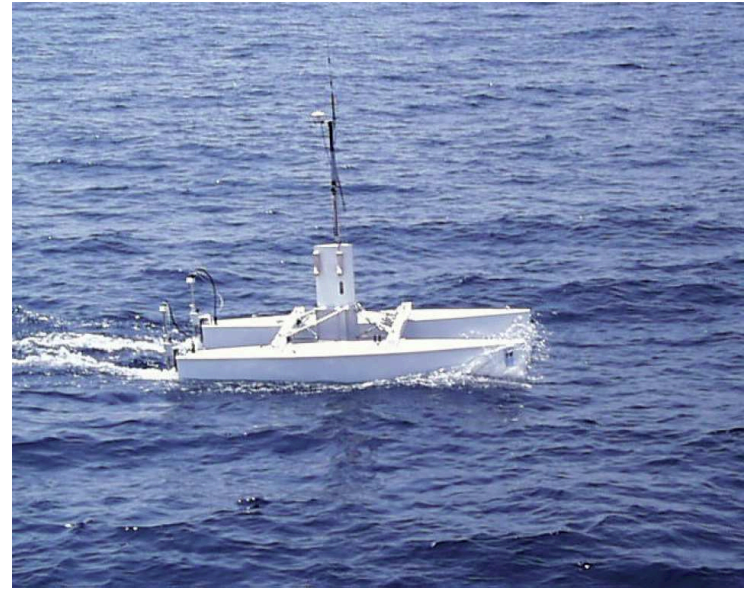

(b) Delfim USV

Figure 1.3: USVs developed at Instituto Superior Téchic (Permission granted by IST)

The Massachusetts Institute of Technology (MIT) pursues its surface aquatic autonomy goals with the Autocat USV [Manley et al., 2000] and the Surface Craft for Oceanographic and Undersea Testing (SCOUT) USV [Curcio et al., 2005]. The $1.8 \mathrm{~m}$ Autocat, shown in Figure 1.5(a), can travel up to $8 \mathrm{~m} / \mathrm{s}$ for 4 hours, 


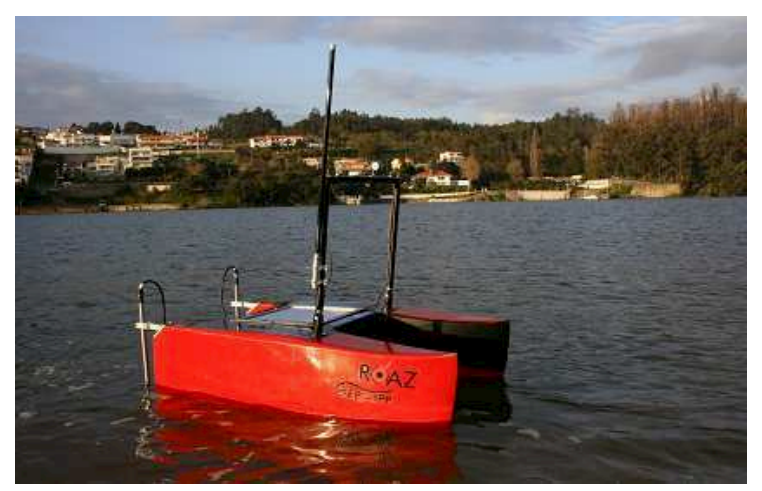

(a) ROAZ USV

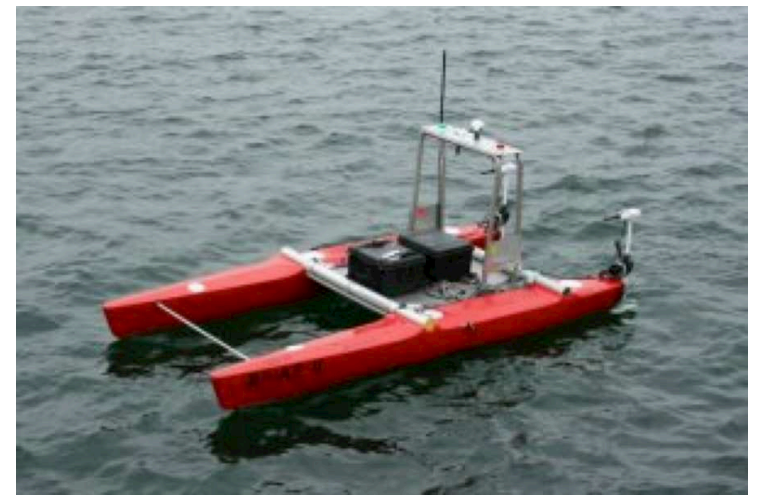

(b) ROAZ 2 USV

Figure 1.4: USVs developed at Instituto Superior de Engenharia do Porto (Permission granted by ISEP)

propelled by a trolling motor or small gasoline outboard motor. Using DGPS and various oceanographic survey equipment the Autocat accomplishes its primary oceanography mission. With the installation of a high frequency sub-bottom profiler, communications link, or SONAR, the Autocat's mission changes to sub-bottom survey, AUV telemetry link, or marine life tracking. Utilizing converted kayak hulls, the low cost SCOUT USVs, shown in Figure 1.5(b), were developed to test cooperative autonomy among multiple agents. Powered using batteries and a trolling motor, the SCOUT USV can travel up to 5 knots. SONAR devices and acoustic communications links can be used for oceanographic surveying and AUV communication. Equipped with GPS and compass devices the SCOUT USVs complete their missions autonomously using waypoint control while actively avoiding collisions with each other.

The Marine and Industrial Dynamic Analysis (MIDAS) research group at the University of Plymouth, UK has developed the Springer USV [Naeem et al., 2006b, Naeem et al., 2006a]. As a 4 m long catamaran the Springer USV, shown in Figure 1.6, has plenty of internal space for the batteries required to drive its two DC trolling motors and to power its navigation and sensor suite, located externally in pelican cases. The vehicle's tracking and control sensor suite includes a GPS, three different compasses (TCM 2, KVH C100, and HMR 3000), a speed log, and depth sensors, all communicating with a Windows PC running Labview via RS232 connections. Additional Windows PCs running MATLAB/Simulink implement guid- 


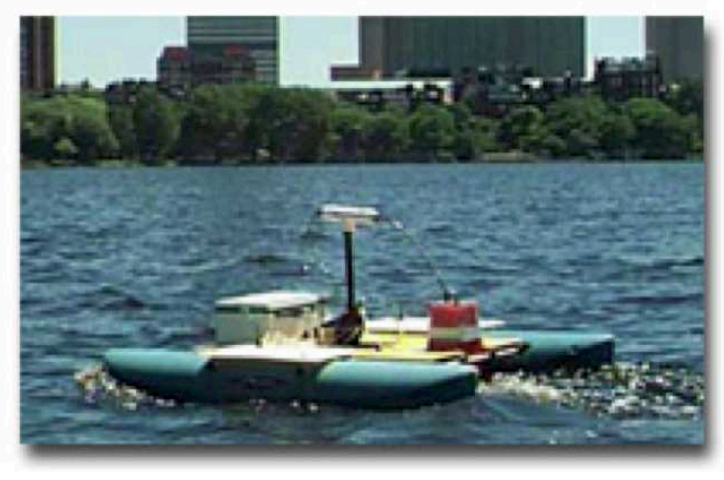

(a) Autocat USV

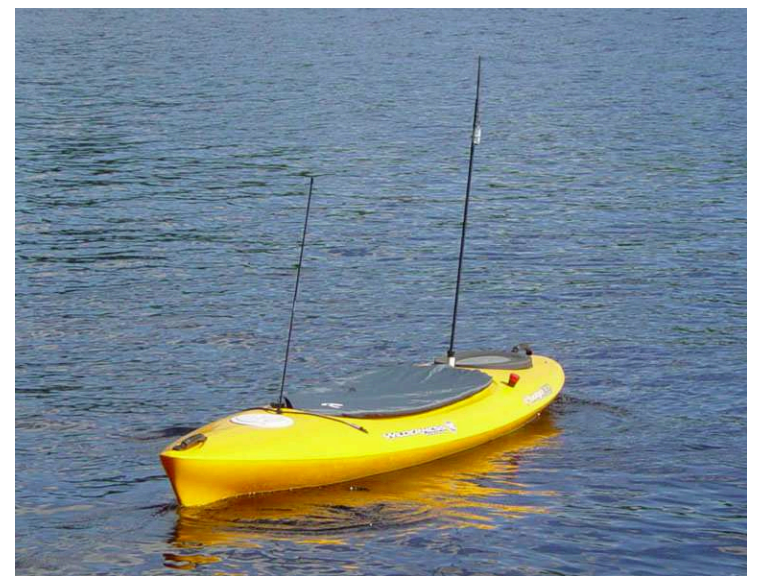

(b) SCOUT USV

Figure 1.5: USVs developed at the Massachusetts Institute of Technology (Permission granted by MIT)

ance and control routines. A two channel RoboteQ AX2850 controls power to the trolling motors yielding speed control and differential steering control. To accomplish its pollution tracking objective the Springer USV has a YSI environmental monitoring unit mounted below the water line between the two hulls. This environmental monitoring unit reads $\mathrm{pH}$, dissolved oxygen, turbidity, temperature, conductivity, ammonium nitrate, chloride, chlorophyll, rhodamine, and oxygen reduction potential levels. Path planning, navigation, and control algorithms designed to track pollution are shown in [Naeem et al., 2008, Naeem W and GN, 2007]. 


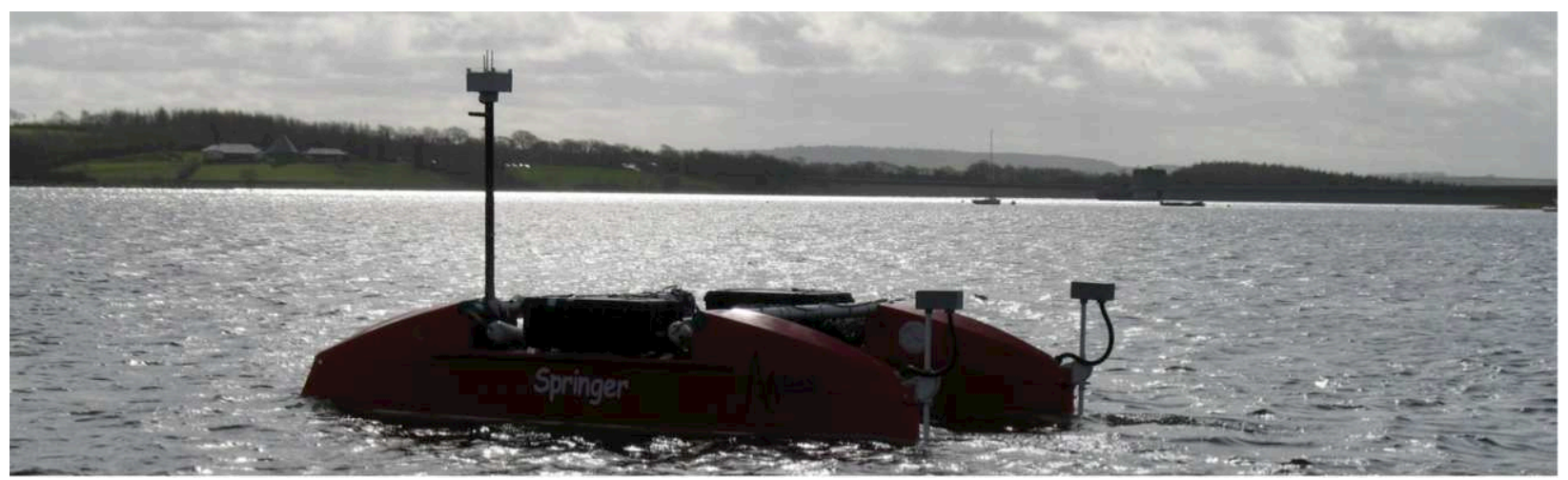

Figure 1.6: The University of Plymouth's Springer USV (Permission granted by UP)

Military and law enforcement companies design purpose built USVs. Most of these vehicles use basic waypoint control or require a remote operator to complete their objectives, though a few outliers are advancing into more autonomous operation. Potential military applications include riverine operations, open water interdiction, harbor patrol, anti-submarine warfare, mine sweeping, as well as target practice.

Israel utilizes the Stingray USV and Silver Marlin USV built by Elbit Systems and the Protector USV built by Rafael Advanced Defense Systems for port defense and terrorist suppression, respectively. The $3.2 \mathrm{~m}$ long Stingray USV shown in Figure 1.7(a) uses water jet propulsion and a $130 \mathrm{hp}$ gasoline engine to propel itself to $50 \mathrm{kts}$. This vehicle comes equipped with a gyrostabilized gimbal containing electro-optical/infrared sensors, a laser marker that auto tracks targets, and a night-cooled thermal imager. Vessel location and orientation information comes from GPS, inertial measurements, and a fluxgate compass. A ground station provides remote control capability though autonomous operation is possible using the aforementioned location and orientation sensors. The Silver Marlin USV shown in Figure 1.7(b) represents the cutting edge in Israeli military USV technology with its autonomous obstacle avoidance capabilities. Powered by two 315 hp marine diesel engines, the $10 \mathrm{~m}$ long Silver Marlin USV has a top speed of 44 kts while carrying a 2.5 ton payload for up to 24 to 36 hour missions. This 2.5 ton payload includes a remotely controlled, stabilized weapon system and an electro-optic naval payload. Adaptive control improves the vessel's operation in rough seas at high speeds. The $9 \mathrm{~m}$ Protector USV shown in Figure 1.7(c) is a remotely controlled stealthy rigid hull inflatable boat that uses water jet propulsion and a diesel engine to travel at speeds of up to 50 
kts. This vessel is equipped for surveillance and targeting purposes like the Stingray USV, however, with a Mini-Typhoon stabilized weapon system the Protector USV also has offensive capabilities.

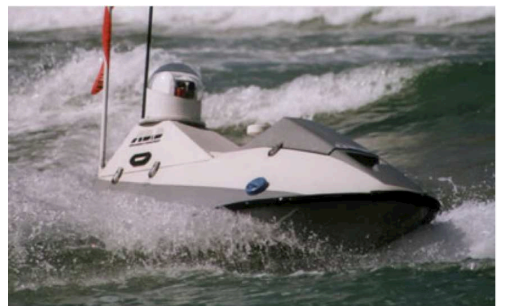

(a) Stingray USV (Permission granted (b) by Elbit Systems)
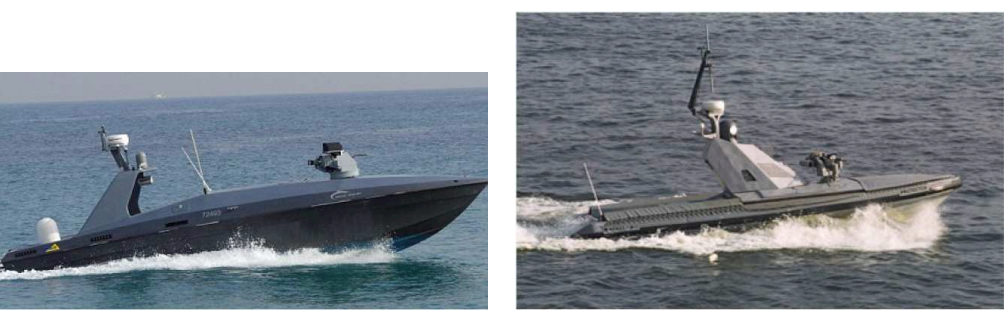

(b) Silver Marlin USV
granted by Elbit Systems)
(Permission (c) Protector USV (Permission granted by Rafael Advanced Defense Systems Ltd.)

Figure 1.7: Israeli military USVs

Canadian USV development includes agile autonomous vehicles used for targeting practice with the Barracuda USV-T and Hammerhead USV-T. The $7.24 \mathrm{~m}$ rigid hull inflatable Barracuda USV-T reaches a top speed of $36 \mathrm{kts}$ due to its $225 \mathrm{hp}$ inline 4 cylinder, after-cooled, marine diesel power plant. Speed, course, and position tracking are accomplished with feedback signals coming from a GPS unit. The Barracuda USV$\mathrm{T}$ is capable of replicating high speed naval tactics by tracking sequences of commands that induce straight line, zig zag, and evasive maneuvers. This vehicle can also be used to sweep for mines and other underwater threats. The $5 \mathrm{~m}$ composite hull Hammerhead USV-T presents the same capabilities of the larger Barracuda USV-T while traveling $4 \mathrm{kts}$ faster with a $135 \mathrm{hp}$ Mercury engine. The Hammerhead USV-T mimics not only maneuvering behaviors but also visual, radar, and laser signatures with its additional radar and visual augmentation systems.

Swedish military development of unmanned vessels spans decades from the SAM mine sweeper introduced in the 1970s to the Piraya USV of today. The the current SAM 3 USV, shown in Figure 1.8(a), is a 20 ton remote controlled or autonomous mine sweeping vessel built by Kockums. Alternative configurations allow the vessel to search for mines with SONAR. The SAM USV has endured the first Gulf War as well as survivability tests that generate massive shockwaves by detonating munitions in close proximity to its hull. At a length of $4 \mathrm{~m}$ the small, agile Piraya USV is the antithesis of the SAM USV. Groups of the $20 \mathrm{kt}, 20$ 
hp vessel shown in Figure 1.8(b) autonomously operate in packs to efficiently patrol coastal waters by using visual/infra-red cameras, radar, communications relays, chemical, biological, radiological, nuclear (CBRN) sensors, loud speakers, and small firearms.

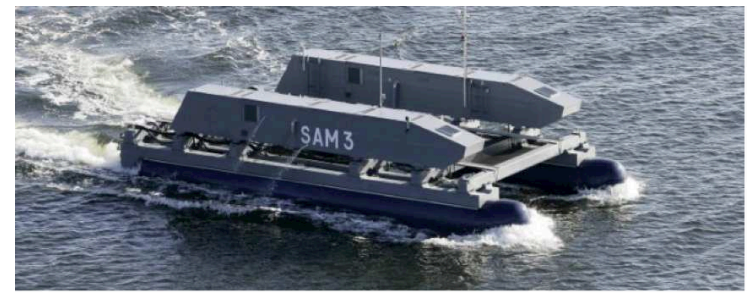

(a) SAM USV

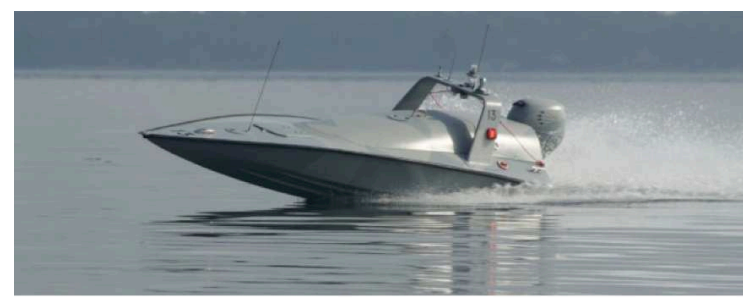

(b) Piraya USV

Figure 1.8: Swedish military mine sweepers and patrol USVs (Permission granted by Kockums)

The British QinetiQ multinational defense company builds the Blackfish USV and Sentry USVs designed for harbor protections and patrol. The Blackfish USV shown in Figure 1.9 can travel at up to 40 kts propelled by its electric motor and battery arrangement while keeping a gimbaled camera on target, which allows a remote operator to remain a safe distance from the situation. This vessel can operate for up to an hour on one battery charge with battery replacement only taking 5 minutes. In addition the Blackfish USV can be outfitted with a gimbaled high resolution SONAR and underwater camera used to classify and identify underwater swimmer threats. The common control unit from the Talon line of robots gives the Blackfish USV heading and waypoint tracking. The newer, water jet propelled, stealthy Sentry USV can carry multiple payloads while traveling at a $50 \mathrm{kts}$ for up to 6 hours. A modular payload framework expands The Sentry USV's mission profile to include unmanned harbor patrol and security, battlefield reconnaissance and damage assessment as well as intruder investigation. The Sentry USV communicates up to a line of sight 16 miles away using a microwave datalink and utilizes a gimbal stabilized high resolution camera and a smoke marker launcher to help accomplish its mission. This vessel has heading and waypoint control similar to the Blackfish USV.

The United States of America has a considerably large footprint in the military USV domain. Well established USV programs include the SSC San Diego USV and Owl USV program. The SSC San Diego 


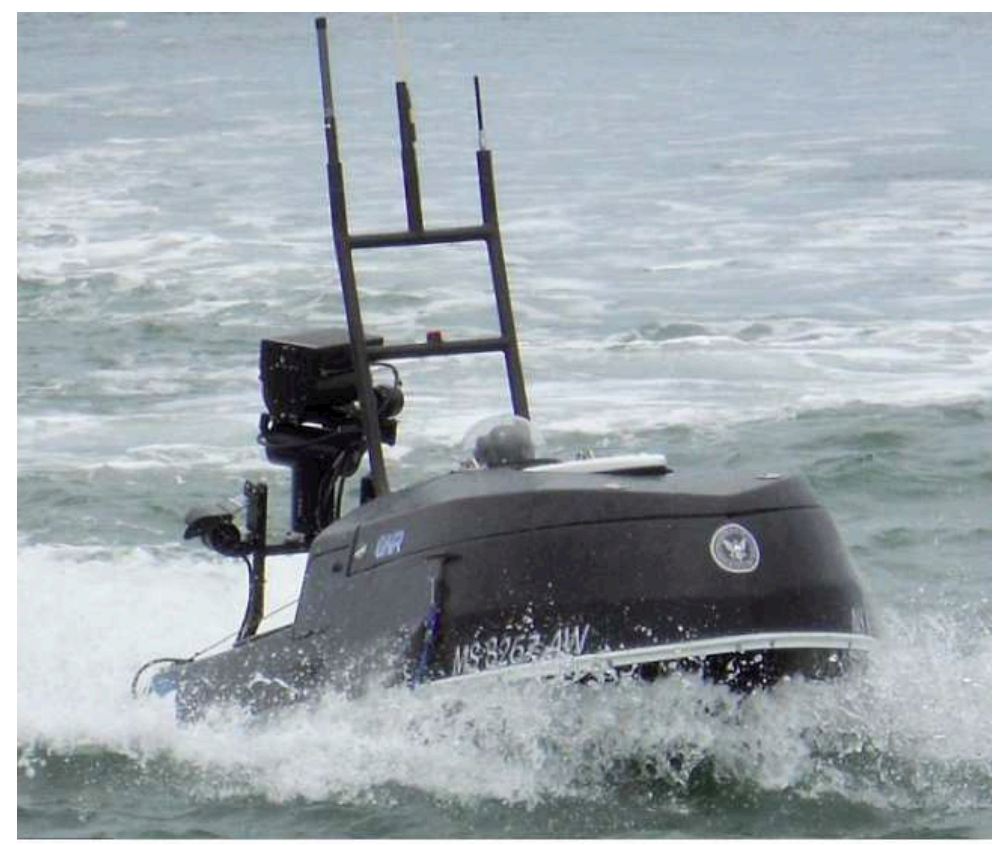

Figure 1.9: Blackfish USV (Permission granted by QinetiQ North America)

USV represents the research side of America's military USVs with original research in obstacle avoidance control algorithms while the recently proposed Draco ASW USV, Textron CUSV, and Piranha USV illustrate the move to equip USVs with heavy armaments usually reserved only for manned operations. The SSC San Diego USV, based out of the Space and Naval Warfare Systems Center in San Diego, is a Seadoo 2000 retrofitted for autonomy in order to operate as a military research testbed. Like many non-military research institutes, this civilian vessel was chosen based upon the ease of installation of sensor and autonomy packages. As a research testbed the SSC San Diego USV, shown in Figure 1.10(a) facilitated research into avoidance of stationary and moving obstacles containing a degree of uncertainty in their of motion, which causes the USV to follow Coast Guard International Regulations for Avoiding Collision as Sea (COLREGS), or "rules of the road" [Larson et al., 2006, Larson et al., 2007]. Missions for the remotely controlled Owl USV mainly consist of riverine and harbor reconnaissance through the use of infra-red and visible light surface sensors (Owl 2) and subsurface sensors (Owl 4).

American USVs currently being designed with heavy armaments understandably have little public litera- 
ture. The anti-submarine Draco USV shown in Figure 1.10(b), showcases an anti-slamming hull design as well as SONAR and torpedo arrays. The Textron CUSV is a large multi-mission vessel with a rear payload bay allowing for autonomous launch and recovery of unmanned submersibles and aircraft. The modular payload bay can also be fitted for anti submarine, mine hunting, communications, and surveillance mission. The Piranha USV concept by Zyvex Marine features a unique hull design that incorporates carbon fiber nano-composites, which allows for exceptionally long range and high payload capabilities.

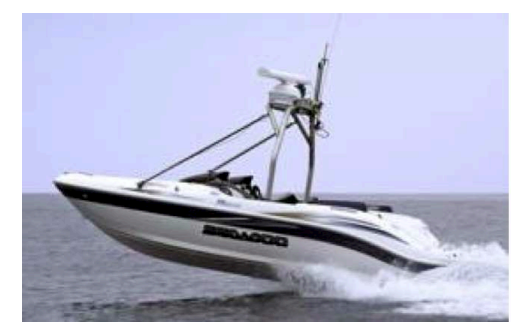

(a) SSC San Diego USV

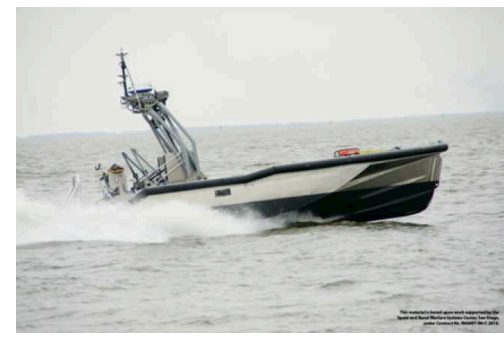

(b) Draco ASW USV

Figure 1.10: American USVs

Autonomous vessel development has grown by leaps and bounds in the past fifteen years. Advanced obstacle avoidance and path planning algorithms unlock a USV's true "fire and forget" potential. To follow these generated paths many control algorithms continue to resort to cross track and waypoint control [Larson et al., 2006, Larson et al., 2007, Naeem et al., 2008]. More general path following [Bibuli et al., 2007, Bibuli et al., 2008, Bibuli et al., 2009, Breivik, 2010, Aircardi et al., 2001, Breivik and Fossen, 2004] and trajectory tracking [Godhavn, 1996, Toussaint et al., 2000, Petterson and Nijmeijer, 2001, Lefeber et al., 2003, Jiang, 2002, Do et al., 2002a, Do et al., 2002b] algorithms have also been developed for vessels with well defined dynamics, but these methods typically involve restrictive assumptions about the dynamic model or the vessel motion (such as "constant speed").

The objective of this study is to devise and implement a control strategy giving a high speed planing vessel the ability to robustly and accurately track aggressive trajectories that span the vessel's entire performance envelope. To fulfill this task a model which is sufficiently rich to enable effective motion planning and control and sufficiently simple to allow straight forward parameter identification must be selected from a set 
of simple models whose parameters can be quickly and easily identified from standard motion data. Having selected an appropriate model, dynamically feasible trajectories can be generated for the USV to follow. An effective closed-loop control algorithm then enables tracking of aggressive trajectories in the presence of environmental disturbances and modeling uncertainties.

\section{Dissertation Overview}

Chapter 2 presents a literature review for surface vehicle modeling, system identification, and motion control. The surface vehicle model review includes both physics based and empirically devised dynamic models describing vessel maneuvering motion. The system identification review spans discrete and continuous model parameter identification in the time and frequency domain. Structured identification methods for linear models that my also be allied to nonlinear systems are discussed. The control review focuses on control algorithms for underactuated vehicles in planar motion.

Chapter 3 presents the Ribcraft USV, the testbed used to obtain system identification and control results for this dissertation. The discussion includes details of the baseline performance capabilities and the hardware used to make the vessel autonomous. The software architecture is presented and closed-loop control implementation is explained.

Modeling is discussed, starting with a general nonlinear six degree of freedom model, in Chapter 4. This most general model is distilled to a three degree of freedom planar motion model, which is then further simplified by observing symmetries present on the research testbed. A vectored thrust bilinear thruster model describes the input forces and moments. Two candidate speed models and three linear steering models, are presented. All of these models incorporate the vectored thrust bilinear thruster model.

Parameter identification methods are shown in Chapter 5. First an algorithm that independently identifies steady state and transient behavior is presented. Required field trials for steering and speed models are then discussed, including the necessary adjustments to identify parameters for bistable sternward behavior. An identification method requiring a specific set of input time histories extracts bilinear thrust parameters from standard motion measurements. System identification results are presented and the best steering and speed models are determined.

Chapter 6 begins with a brief definition of trajectory tracking error dynamics. A baseline PD cascade tra- 
jectory tracking control law and its stability properties are presented. An augmentation of the backstepping control law presented in [Jiang and Nijmeijer, 1997, Jiang et al., 2001] allowing for more precise gain tuning is then shown. Local exponential convergence is proven for the backstepping closed-loop time-varying system. By backstepping the control law an additional time convergence is proven for the steering and speed models selected from Chapter 5. Including parameter scheduling that accounts for disparities in planned and actual model parameters yields a field implementable backstepping trajectory tracking control law that also controls the bistable steering behavior prevalent in sternward motion. Finally, this backstepping control law is augmented to account for constant (or slowly varying) flow conditions. Trajectory tracking performance results of the PD cascade and backstepping control law are then compared.

Conclusions and an overall assessment of the identification and control strategies are shown in Chapter 7.

\section{Contributions}

- An identification method to find parameters of a bilinear thruster model using only vessel motion data.

- Development and validation of a speed-scheduled variable structure dynamic model for a planing vessel.

- Identification of a vessel's bistable sternward motion behavior.

- Development of a nonlinear backstepping controller that causes a high speed planing vessel to track trajectories spanning the vessel's entire forward and sternward speed envelope.

- Augmentation of the nonlinear backstepping controller to compensate for steady and slowly varying fluid flow conditions. 


\title{
CHAPTER
}

\author{
2
}

\section{BACKGROUND}

This chapter reviews theory that relates to the remainder of the dissertation, providing context for results. Section 2.1 presents an overview of models describing displacement vessel maneuvering motion, developed through study of the underlying physics and observation through experimentation. Section 2.2 examines methods of identifying parameters for linear models by minimizing error metrics in the time or frequency domain. Section 2.3 describes path following and trajectory tracking methods that are applicable to vessels.

\subsection{Modeling}

Fossen [Fossen, 1991] provides a review of surface vessel motion models. In addition to the six degreesof-freedom (DOF) nonlinear motion model, he discusses a variety of "maneuvering" models that describe a vessel's motion in the horizontal plane. These are generally based on a combination of physical principles and empirical observation, and they are often decomposed into speed (surge) and steering (sway and yaw) 
models.

The model of Abkowitz [Abkowitz, 1964] combines the planar rigid body equations with a third order truncated Taylor series expansion of the hydrodynamic forces and moments about a steady straight line motion. A few simplifying assumptions are made to limit the number of model parameters:

- Only first order acceleration terms are included

- The vessel has port to starboard symmetry

- Velocity and acceleration coupling is neglected

The model of Norrbin [Norrbin, 1970] incorporates planar rigid body equations with an exclusively empirical representation of hydrodynamic forces and moments, based on detailed observations of scaled model tow-tank experiments. Norrbin's model also includes comprehensive modeling of effective rudder angle as well as thrust modeling, absent in Abkowitz's model. A simplified version of Norrbin's model is described by Blanke [Blanke, 1981].

The simplest and most popular steering model is the first order turn rate model described in [Nomoto et al., 1957]. Although the model was developed for displacement vessels, it has been applied to semi-planing and planing vessels, as well. A deficiency of Nomoto's model is its inability to accommodate slipping motion, an appreciable effect in some operating regimes. Nomoto's first order steering model is expanded by including a first order equation for sideslip [Yu et al., 2008a] This "Nomoto-with-sideslip" model is of the same order as the linear steering model obtained by linearizing about steady forward motion, but with fewer parameters; in transfer function form, the Nomoto-with-sideslip model omits a zero that appears in the linearized steering equations.

Rolling motion can also be included in a vessel steering model, at least in scenarios where pitch and heave decouple from the lateral modes [Amerongen and Cappelle, 1981, Son and Nomoto, 1981, Christensen and Blanke, 1986]. The model of Christensen and Blanke [Christensen and Blanke, 1986] assumes that the forces and moments involve square terms in surge velocity. This formulation includes rudders, propellers, and bow thrusters lumped into a single roll moment term. While the vessel considered in the present work undergoes appreciable rolling motion while maneuvering in calm water, the effect of rolling motion on the 
steering motion can be effectively incorporated into a simpler, planar motion model.

\subsection{Parameter Identification}

Parameter identification involves determining values for parameters in a structured model of a given system such that input/state histories of the model best match input/state histories of the physical system. The model can be based on physical insight concerning the actual system or it can take on a more general structure, defined by some distribution of basis functions. The model can be linear or nonlinear and can evolve in discrete or continuous time.

System identification is a mature field with an extensive body of literature. There exist several authoritative textbooks on the topic [Jategaonkar, 2006, Ljung, 1999, Juang, 1994, Morelli and Klein, 2006]. In general, model parameters are determined by minimizing a definition of error between the true and predicted state histories, for given input histories. A standard approach to minimize the error is least squares regression. Variations on this approach include the instrumental variable method [Wong and Polak, 1967], singular value decomposition, which is used in the Eigenvalue Realization Algorithm (ERA) in [Juang, 1994], stochastic approximation, and a variety of others.

Though all physical systems exhibit some degree of nonlinear behavior, only linear time invariant (LTI) system identification is discussed here. Frequency domain identification is a common approach for LTI systems [Morelli, 2000b]. In the frequency domain, the parameters to be identified are the coefficients of the numerator and denominator polynomials in the input/output transfer function. A number of identification methods compare frequency domain signal error between the identified model and experimental data; some use the 2-norm [Bayard, 1994b, Bayard, 1994a, Sanathanan and Koerner, 1963, Klein, 1978, DuVal, 1981, Morelli, 2000b, Theodore et al., 2004, Wang and Zhang, 2001, Lin and Wu, 1982, Morelli, 2000a, Selvam et al., 2005] while others use the $\infty$-norm [Jacobson and Tadmor, 1993, Helmicki et al., 1991, Partington, 1992, Gu and Khargonekar, 1992, McKelvey et al., 1996] of the error. Advantages of identifying system parameters in the frequency domain include the ability to truncate the measured frequency response, the explicit capturing of system modes, and the lack of need for numeric differentiation. Truncation of the measured frequency response to the frequency range of interest yields system identification uncontaminated 
by high-frequency disturbances. By identifying parameters based upon frequency data, prominent system modes can be easily identified. Finally, since all frequency domain identification is done in the frequency domain, time derivative of noisy data unnecessary. The primary disadvantages of frequency domain analysis include the required application of a discrete Fourier transform on measured data, the constraint that only linear systems can be identified, and the difficulty of attempting to identify heavily damped systems.

Time domain identification methods are also used for LTI systems [Jategaonkar, 2006, Hamel and Jategaonkar, 1996, Klein, 1989], and common applications include the determination of aerodynamic or hydrodynamic coefficients [Sri-Jayantha and Stengel, 1988, Stalford, 1981, Yoon et al., 2004, Yoon and Rhee, 2003]. Identifying continuous time [Rao and Unbehauen, 2006] models requires the computation of signal derivatives while identifying discrete time models does not.

In the continuous time domain, the measured derivative of state is compared with the estimated derivative of state. However, acceleration signals directly measured or deduced from noisy velocity data are excessively noisy in general. These noisy signals must be subjected to a smoothing process, such as the Savitzky-Golay algorithm described in [Savitzky and Golay, 1964], in order for the identification algorithms to converge to correct system parameters. The Savitzky-Golay algorithm, which fits third order or greater polynomial coefficients to a signal, intrinsically gives first and second derivatives of smoothed data. A primary advantages of time domain system identification is its applicability to nonlinear systems.

Optimization methods are required to determine system parameters that minimize modeling error. Offline "batch" optimization methods that directly find global minima include least square regression [Stengel, 1986], the instrumental value method [Wong and Polak, 1967, Young, 1970, McKelvey, 1997, Gupta, 1978, Zimmermann and Mingori, 1996, Stoica and Soderstrom, 1983], which augments the least squares regression if the state and noise are correlated, and singular value decomposition, which is required for ERA implementation. Iterative offline optimization methods that start at an initial parameter guess and search for a potentially local minimum include the gradient descent method, Newton-Raphson method, Gauss-Newton algorithm, Dantzig simplex method, Nelder-Mead simplex method, neural network algorithms [Narendra and Parthasarathy, 1990,Levin and Narendra, 1993, Kosmatopoulos et al., 1995, Levin and Narendra, 1996, Chen et al., 1990, Chen and Billings, 1992], and the stochastic approximation method [Sakri- 
son, 1967, Kiefer and Wolfowitz, 1952, Robbins and Monro, 1951]. In this work, least squares regression is used to identify parameters.

This review has shed light on the vast variety of parameter identification techniques though the primary goal of vessel parameter identification has not been specifically addressed. Parameter identification methods applied to surface vessels include frequency domain approaches [Selvam et al., 2005] and time domain approaches [Källström and Åström, 1981, Åström and Källström, 1976, Muske et al., 2008, Hayes, 1971, Mišković et al., 2011]. Optimization of parameters is done using hybrid-extended Kalman filtering [Yoon and Rhee, 2003, Yoon et al., 2004, Hayes, 1971, Fossen et al., 1996], recursive least squares [Nguyen, 2008], and artificial neural network (black box) methods [Rajesh and Bhattacharyya, 2008]. Note that all identification methods require parameter optimization, though the specific algorithms are often not explicitly stated. Two overviews of marine system identification methods give insight into system identification methods used currently [Ødegård, 2009] and in the past [Brinati, 1973].

\subsection{Trajectory Tracking}

Path following for nonholonomic and underactuated vehicles [Bibuli et al., 2009, Sordalen and Canudas de Wit, 1993, Soetanto et al., 2003, Micaelli and Samson, 1993, Aircardi et al., 2001] provides convergence to curves in space, though not necessarily convergence to a time-parameterized trajectory. Trajectory tracking controllers drive the full state of a system to a desired time-varying reference state.

Development of trajectory tracking control laws using inertial Cartesian position error in conjunction with nonlinear backstepping is straightforward. By taking the time derivative of a candidate Lyapunov function containing the inner product of Cartesian position error, a virtual input can be defined that makes the Lyapunov function's time derivative negative. This method is repeated until the forward force input is encountered in a Lyapunov time derivative. Defining a forward force control law to cancel out the top value of the only non-negative 2-vector in the inner product in the Lyapunov function allows an additional virtual input to be defined for the bottom value of the 2-vector. Backstepping a final time exposes the turn moment input, which is defined to give a negative Lyapunov function, yielding exponential convergence to a trajectory [Godhavn, 1996]. Unfortunately the vehicle's velocity must remain non-zero, which cannot 
be guaranteed by trajectory selection, and forces and moments must be directly applied. Modifying this backstepping method has made it possible to to overcome non-minimum phase issues brought on by defining the inputs more realistically as forces output from a rear thruster [Toussaint et al., 2000], though the non-zero vehicle velocity requirement remains.

Body fixed Cartesian trajectory tracking control laws avoid the non-zero vehicle velocity constraint found in inertial Cartesian frame trajectory control. The value of avoiding this inadequacy is essential since manipulation of the reference trajectory cannot guarantee non-zero vehicle velocity. However, reference trajectories, which one can easily manipulate, must not contain instances of zero velocity. Early results using body fixed position error in trajectory control take advantage of kinematic relationships to yield global asymptotic stability and local exponential stability of a wheeled robot [Kanayama et al., 1990]. This control law is modified to include path following [Fierro and Lewis, 1995] and stabilization by utilizing the averaging approach that yields a smooth transition between trajectory tracking and stabilization [Pettersen and Nijmeijer, 1999]. A control law exploiting backstepping and boundedness of the sine of a term divided by that term yields global asymptotic stability [Jiang and Nijmeijer, 1997], which is expanded to include saturation phenomena [Jiang et al., 2001]. Exploiting the triangular structure of the error variables coming about due to a transformation to the body frame. combined with an additional transformation, yields exponentially stable vessel dynamics when recursive vector backstepping is used [Petterson and Nijmeijer, 2001]. Both inertial and body fixed Cartesian formulations yield trajectory tracking at the expense of exceedingly complex controller structure. Simplification of controller structure can be accomplished using body fixed Cartesian error fashioned into a cascade system.

A cascade approach to trajectory tracking first proposed for mobile robots [Panteley et al., 1998] sets three conditions that yield global uniform asymptotic stability for a time varying cascade system, which is then expanded for exponential stability. A cascade system is one where the inner subsystem is additively perturbed by the state of an outer subsystem multiplied by a function containing inner and outer system states. A key feature of the cascade system is independence of the outer subsystem from the inner subsystem. Using a body fixed Cartesian error frame two independent controllers command turn rate to induce heading convergence and forward speed to induce along-track error convergence. Note that cross-track error never 
enters the formulation. If reference turn rate is persistently excited (PE) these two independent controllers put the system into cascade form giving asymptotically stable trajectory tracking. This control formulation is augmented to provide global asymptotic stability for vessel dynamics [Lefeber et al., 2003]. Including backstepping into the cascade approach for vessels gives exponential convergence [Jiang, 2002]. However, the restrictive condition of PE reference turn rate remains. By introducing an intermediate "virtual" reference turn rate and speed used to give non-zero reference turn rate [Do et al., 2002a], the PE condition is lifted allowing convergence to straight sections of reference trajectories, which is then further augmented to give simultaneous trajectory tracking and stabilization [Do et al., 2002b]. 


\section{CHAPTER}

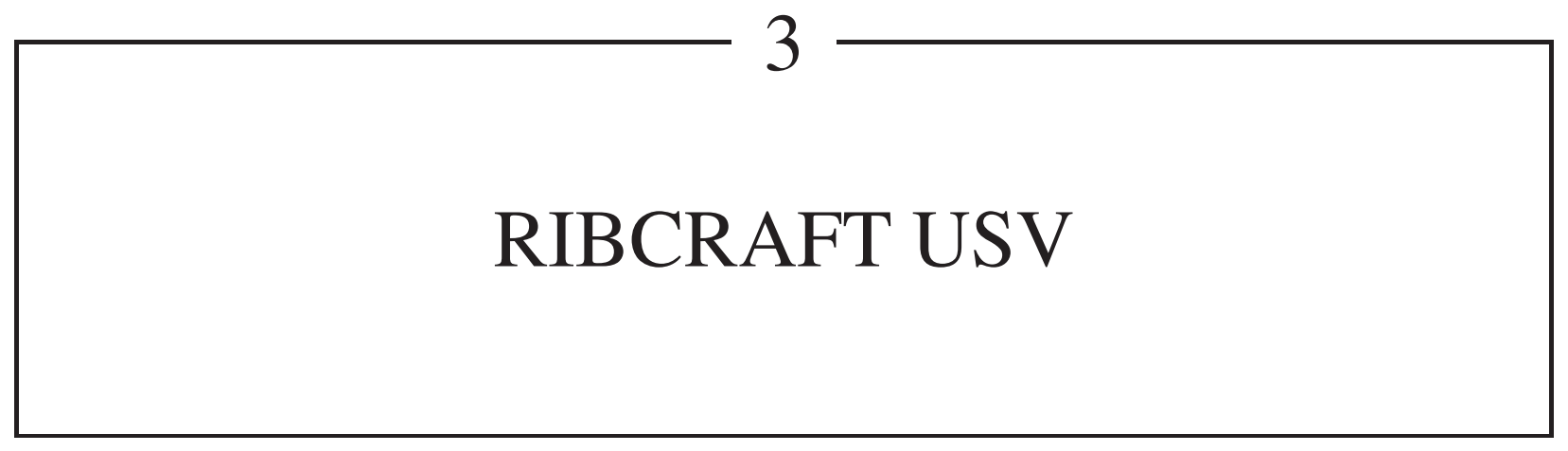

The Autonomous Systems and Controls Laboratory's (ASCL) mission of demonstrating mapping, trajectory planning, and control algorithms for riverine environments calls for a capable autonomous vessel. Requirements include quick construction to expedite mission readiness, durability to handle aggressive intended and potentially unindented actuation, dynamic stability to keep electronics and possible occupants safe, ease of maintenance to prevent downtime, and space for multiple occupants for debugging and preliminary testing stages of development. Designing and constructing a vessel is too costly and time consuming, so an existing model is chosen and converted for autonomous operation. The rest of this section discusses the performance and capabilities of the selected vessel and the hardware and software used to make it autonomous. Literature related to the vessel and its autonomy package includes [Gadre et al., 2012a, Gadre et al., 2009, Xu et al., 2009a, Xu et al., 2009b, Xu et al., 2010, Xu et al., 2012, Sonnenburg et al., 2010, Stilwell et al., 2011, Sonnenburg and Woolsey, 2012, Sonnenburg and Woolsey, 2013, Gadre et al., 2012b]. 


\subsection{The Ribcraft 4.8}

The rigid hull inflatable Ribcraft 4.8 best fits the criteria stated above. With its deep V-hull the Ribcraft 4.8 has excellent handling qualities. The vessel exhibits minimal skidding in turns, planes with ease, and remains stable throughout its performance envelope. The deep V-hull coupled with high bow sheer has the added benefit of superior sea-keeping abilities in rough seas. The vessel's rigid hull is constructed of fiberglass with a full width composite stringer system to increase strength. The inflatable tubes made of Hypalon resist damage due to impact, preventing mishap due to botched docking, while simultaneously providing extra buoyancy for sea-keeping. Due to its light weight this vessel is easily transported and launched with a trailer. The particular Ribcraft 4.8 used by the ASCL is equipped with a 50hp four-stroke Honda outboard motor propelling the vessel to a top speed of 22 knots.

\subsection{The Autonomy Hardware}

Outfitting this capable stock vessel for autonomy first requires additional hardware to actuate the thrust angle and throttle electronically, giving remote control capabilities. Sensing devices must then be mounted that feed information to closed loop control and trajectory planning algorithms. With the hardware in place, an in-house software package gives the ability to control actuators using open loop commands or simple closed loop controllers, which track desired propeller rpm, speed, heading, or location. Modifying these controllers or developing more advanced nonlinear trajectory tracking control laws built on the existing software architecture expands the capabilities of the autonomous vehicle. Data recorded from the sensing devices by the software package gives the ability to identify vessel dynamic motion and to debug mapping, planning, and control algorithms. Additionally, the software package contains a vessel simulator allowing for exhaustive debugging of complicated algorithms, reducing wasted time spent in the field.

\subsubsection{Input Control Hardware}

The Ribcraft's battery, which is charged by the outboard motor's alternator, powers all hardware. This 12 volt supply passes through a DC MegaPAC DC to DC switcher made by Mission Power Solutions with 
Vicor components that converts all power to a 12 volt and 5 volt power line. These power lines first pass through a relay board that acts as an onboard emergency stop. When the emergency stop button is pressed the signal voltage keeping the relays closed is cut, causing the relays to open the circuit, which cuts power to the actuators. The vessel has off board E-stop capabilities by using a Torc SAFESTOP transmitter in conjunction with a Torc SAFESTOP receiver tied to the ignition. When the emergency button is pressed or when the transmitter-receiver radio link is lost the onboard receiver cuts power to the spark plugs, which shuts the engine off but leaves throttle position and thrust angle actuation unaffected. After traveling through the relay board, the power lines pass through a fuse board, which prevents excessive amperage from reaching the sensitive actuator components. Figure 3.1 shows the Vicor, relay board, and fuse board, Figure 3.5 shows the onboard emergency stop, and Figures 3.2(a) and 3.2(b) show the Torc wireless emergency stop.

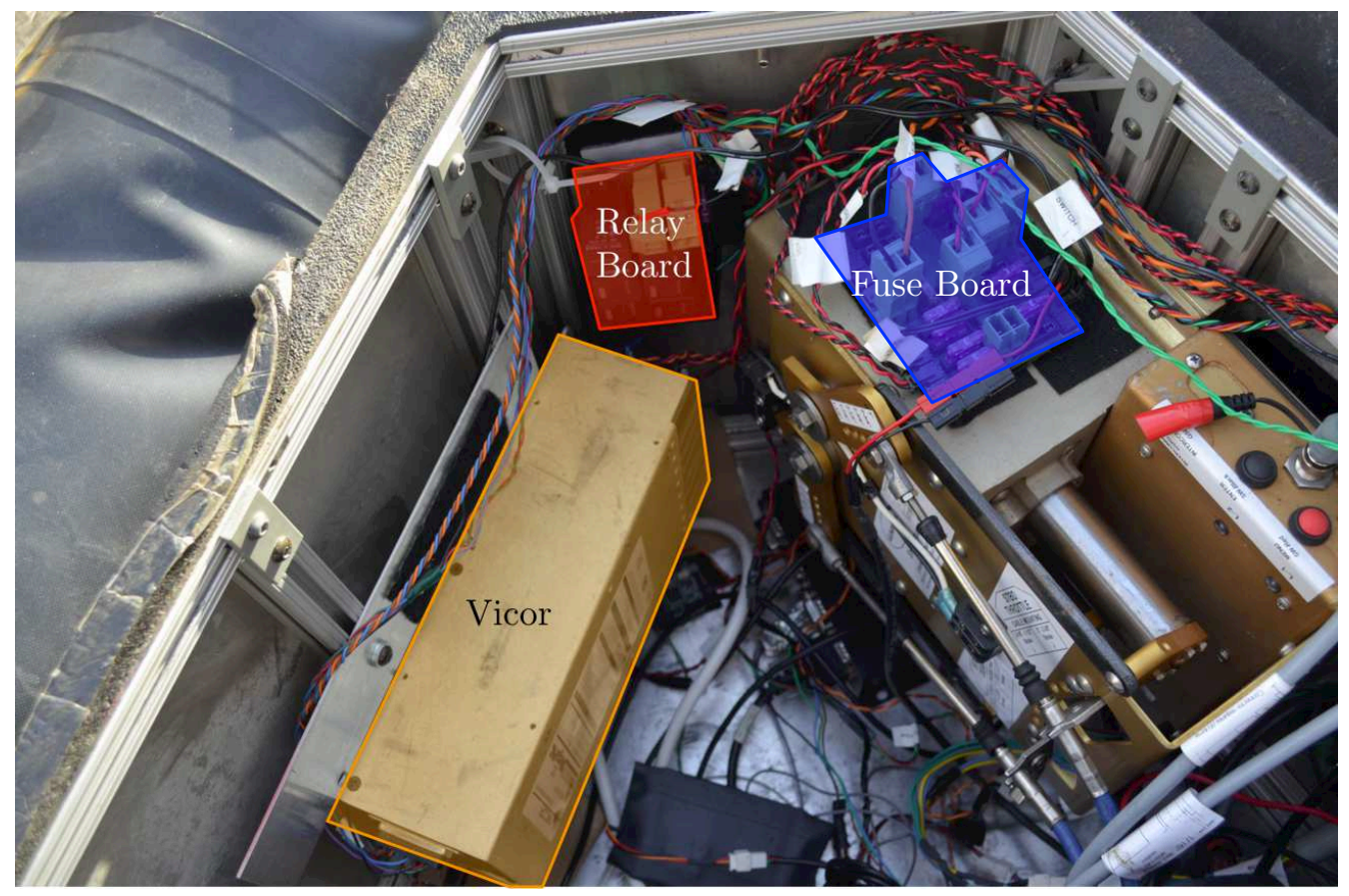

Figure 3.1: The Vicor, relay board, and fuse board in the Ribcraft USV's electronics box

A M81120 Raymarine hydraulic pump incorporated into the power steering system drives turning motion 


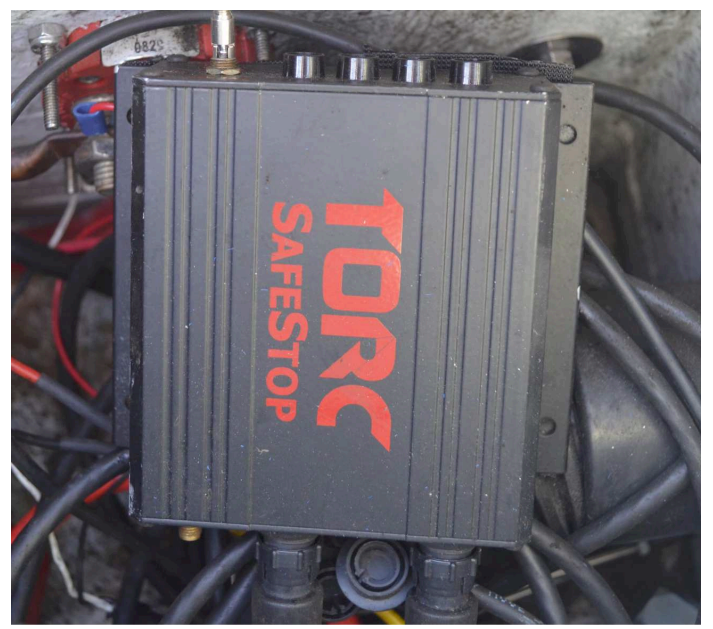

(a) Torc onboard receiver

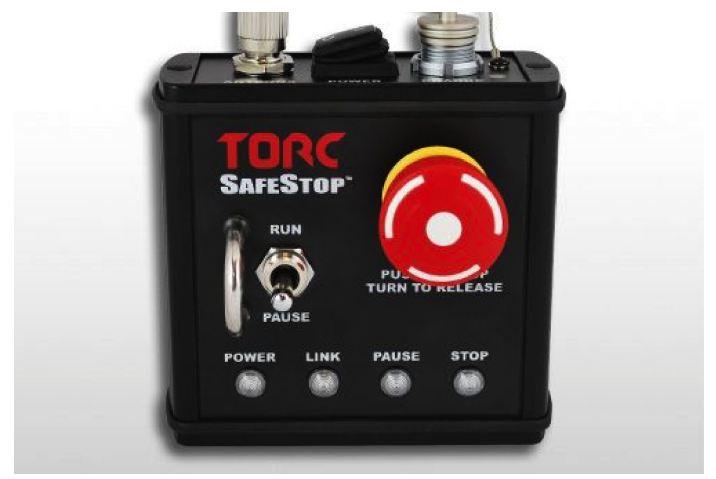

(b) Torc off board transmitter

Figure 3.2: The Torc wireless E-stop

of the outboard engine. This particular pump has a maximum stall pressure of $750 \mathrm{psi}$, which defines the rate limit for actuation. Variable amperage up to 60 A from a RoboteQ AX500 motor controller provides power to a hydraulic motor capable of sourcing $5 \mathrm{~A}$ to orient the outboard engine to the commanded angle. This motor controller is capable of receiving open loop speed control as well as closed loop speed or position control through a serial port input, radio-control pulse-width input, or 0 to $5 \mathrm{~V}$ analog input. A Raymarine position sensor measures the angle of the outboard motor relative to the vessel, allowing closed loop outboard orientation using the motor controller's built in PID controller. This position sensor outputs a voltage between 0 and $5 \mathrm{~V}$, however, full deflection of the outboard engine only nets a voltage between 2.1 and $2.9 \mathrm{~V}$ yielding low resolution position control. Furthermore the PID gains, constrained by maximum values of 10 , underutilize the available power of the motor controller. Using four op amps for a combination of voltage following, offsetting, and amplifying transforms the voltage sweep from 2.1 to $2.9 \mathrm{~V}$ to 0 to 5 $\mathrm{V}$, recovering position resolution and motor controller utilization. Figure 3.3 shows the op amp board, and motor controller installed in the electronics box while Figure 3.4 shows the position sensor installed next to 


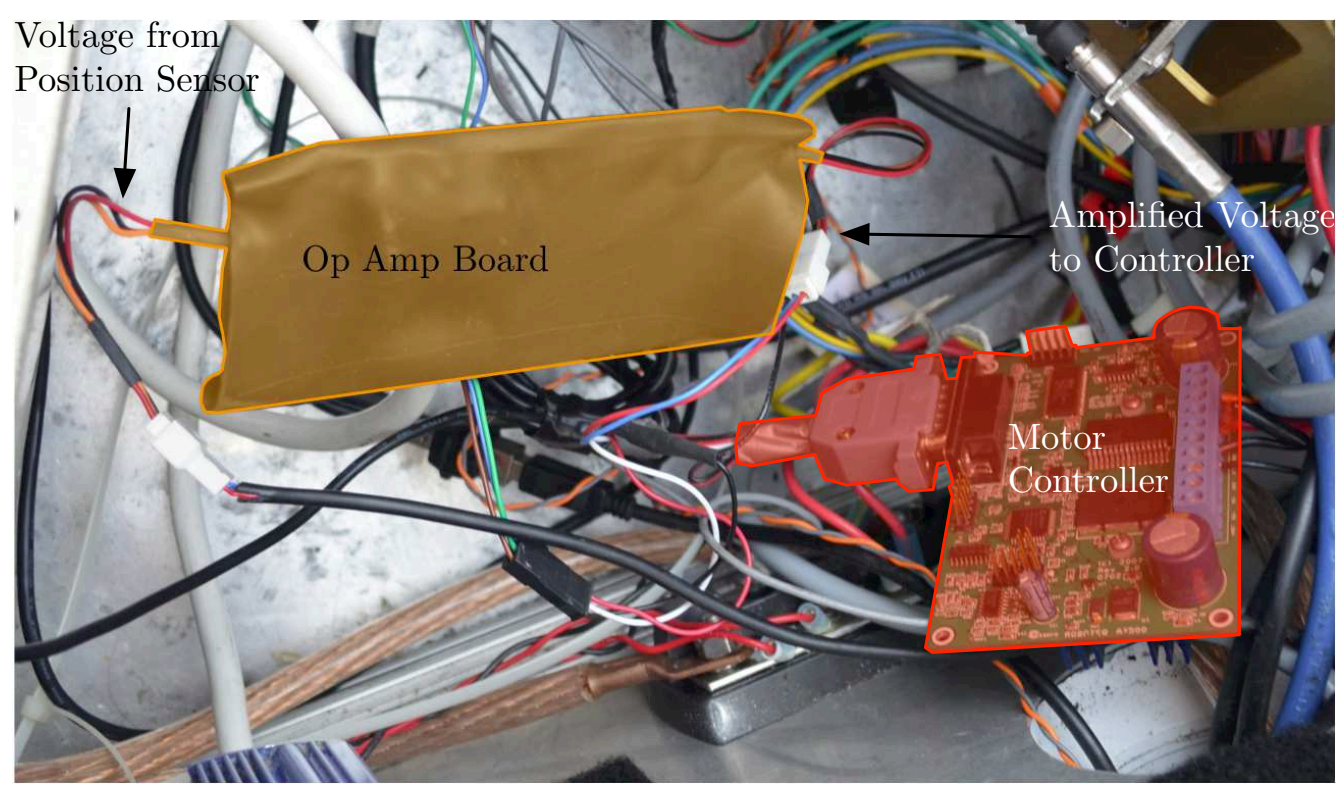

Figure 3.3: The op amp board and motor controller

the outboard motor.

Glendinning devices actuate the engine throttle and gear assemblies for remote and manual operation. Throttle and gear adjustments on the stock Ribcraft are made from the cockpit by using a lever that pushes and pulls two shrouded metal cables. A Glendinning Smart Actuator uses powerful electric motors to push and pull these cables based upon forward and reverse throttle commands. These throttle commands come from an electronic throttle lever for manual control or an Analog Control Interface that accepts an analog voltage from 0 to $5 \mathrm{~V}$ for computer control. For the Analog Control Interface a voltage of $2.5 \mathrm{~V}$ gives the neutral throttle position while higher voltages give forward throttle and lower voltages give reverse throttle. In order to electronically control throttle, a digital potentiometer accepts hex values from 0 to FF through a serial connection causing one of 256 different voltages spanning 0 to $5 \mathrm{~V}$ to be output. Figure 3.7 shows the Smart Actuator and Analog Control Interface, Figure 3.6 shows the digital potentiometer, and Figure 3.5 shows the electronic throttle lever. 


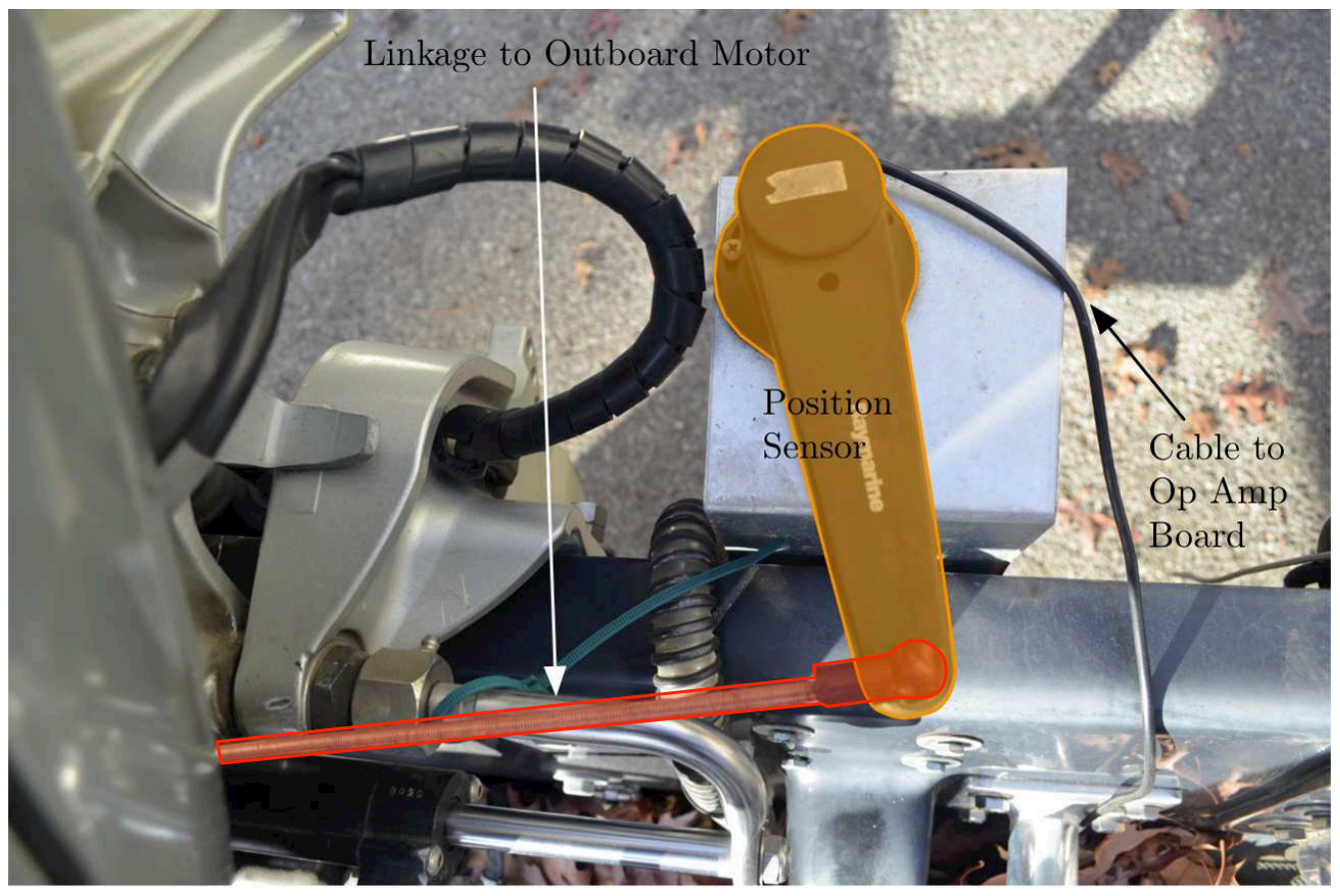

Figure 3.4: The Raymarine position sensor for steering feedback

\subsubsection{Sensor Hardware}

A PIC30F3011 Microchip programmable microcontroller measures engine RPM and consequently propeller RPM. The microcontroller intercepts one of the four engine spark plugs' 12 volt pulses that are normally destined for the stock RPM gauge. Before entering the microcontroller two resistors scale the voltage pulses to 5 volts to conform to the chip's TTL standard. The internal oscillator rated at 7.37 Mhz, used for internal timing and accurate quadrature calculations, calculates time between pulses. This internal oscillator triggers an interrupt to the quadrature timer, immediately yielding the time since the last interrupt for every rising $5 \mathrm{~V}$ edge coming from the raw RPM pulses. Period information is transformed into an engine RPM value and saved. The same internal oscillator also triggers an interrupt at $10 \mathrm{~Hz}$ to send the saved engine RPM value along a serial RS232 at a baud rate of 115200 , which enables a connected computer to both log engine RPM information as well as instantaneously use it for control purposes. This device, pictured in Figure 3.8 


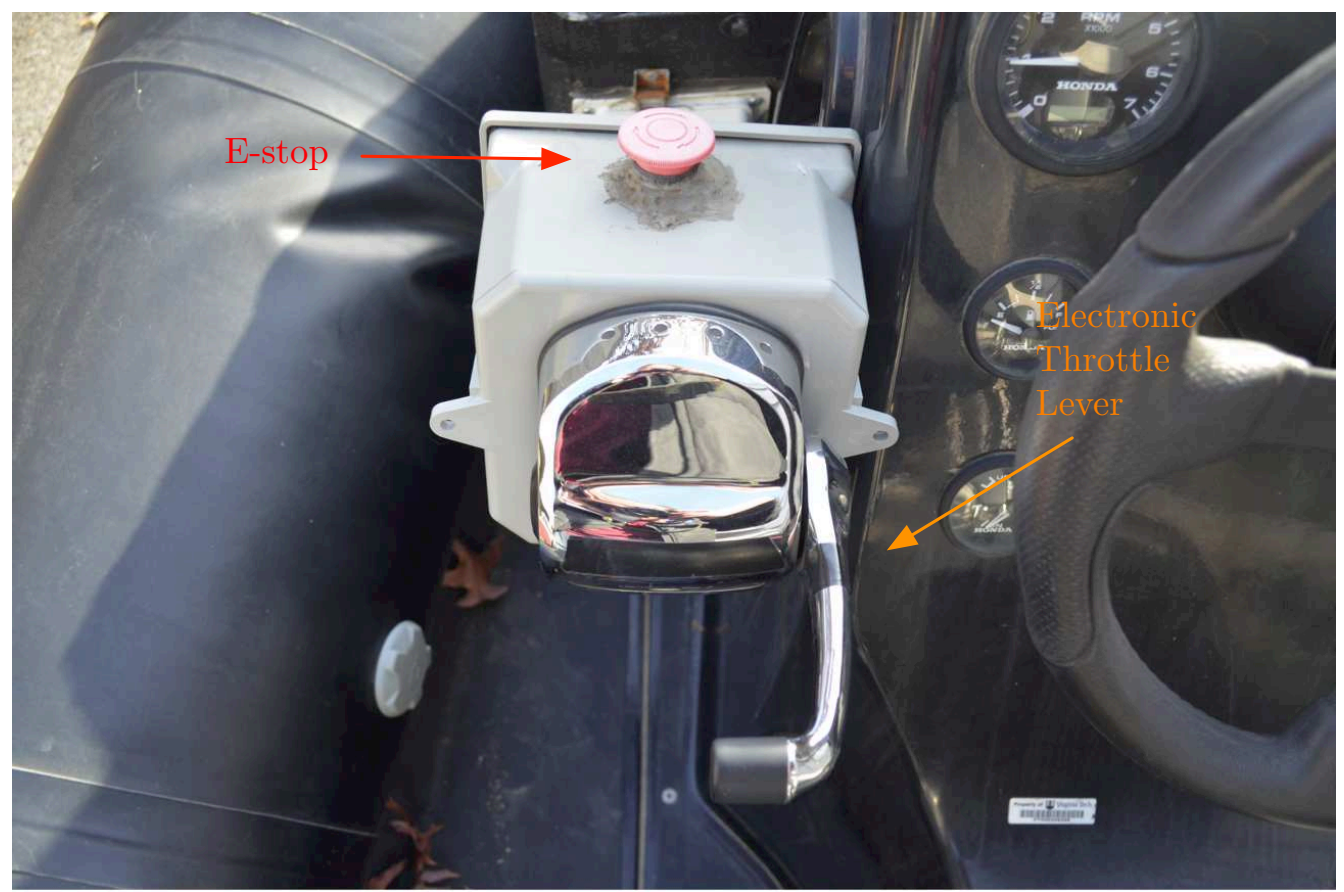

Figure 3.5: The emergency stop and electronic throttle lever

operates at all times when the circuit to the boat battery is closed.

A ComNav Vector G2 DGPS compass mounted on aluminum bars located at the stern of the Ribcraft USV, as shown in Figure 3.9, measures position and orientation. The Vector G2 can interface with vessel navigation equipment using the NMEA 0183 standard via two independent RS-422 ports or through two standard serial RS-232 ports. This GPS device is highly configurable with a multitude of different NMEA 0183 and proprietary output messages giving satellite fix data, satellites in view, pseudo range error statistics, active satellites, range residual, true heading, magnetic heading, rate of turn, course and speed over ground, and time and date information. These output messages can be individually enabled and disabled, which reduces message clutter and increases the peak message output rate for a given baud rate. Internal filters with configurable time constants can smooth turn rate and heading, though this capability is disregarded in lieu of filters implemented in the control software. Since the Vector G2 is a DGPS device, position information is enhanced by satellite corrections sent from a nearby base. As previously stated, this device also outputs true 


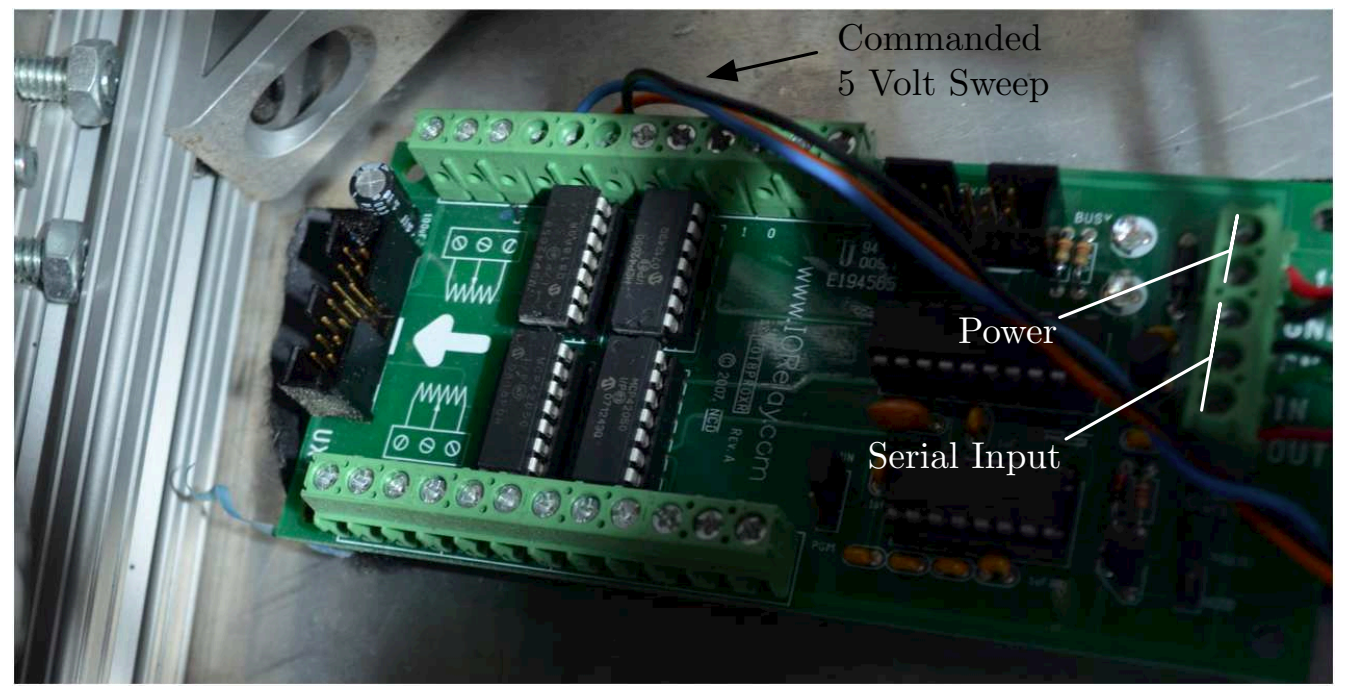

Figure 3.6: The digital potentiometer converting serial commands into analog voltage

heading and turn rate information by utilizing two internal GPS receivers. Instead of computing heading by comparing position information of the two GPS receivers, which would yield inaccurate and noisy measurements, the phase shift of the satellite radio signal, measured by the two GPS receivers, gives a relative orientation between the device from the signal source. Adding the absolute angle of the GPS satellite from North gives the true heading of the device. Magnetic fields and local magnetic deviations, which degrades uncalibrated AHRS measurements, do not affect the Vector G2's heading and turn rate measurements.

The Ibeo Alasca laser line scanner and electronic control unit (ECU) detects obstacles and shorelines. The laser line scanner uses four infrared lasers whose beams reflect off of an angled spinning mirror and onto objects around the device. These lasers are mounted with small incremental differences in vertical angle giving a band of object detection along the horizon as the laser beams spin. Up to four "echoes" or laser reflections can be measured for a given laser pulse, allowing detection of multiple, potentially semitransparent, objects by a single laser pulse. This echo collecting method of range finding does not see obstacles that reflect the original pulse away from the device, meaning laser scans of bodies of water do not elicit false detection of obstacles. A purpose built ECU must be used to compute range from the collected laser measurements due to the exceptionally small times between pulse and reflection. Ibeo's proprietary 


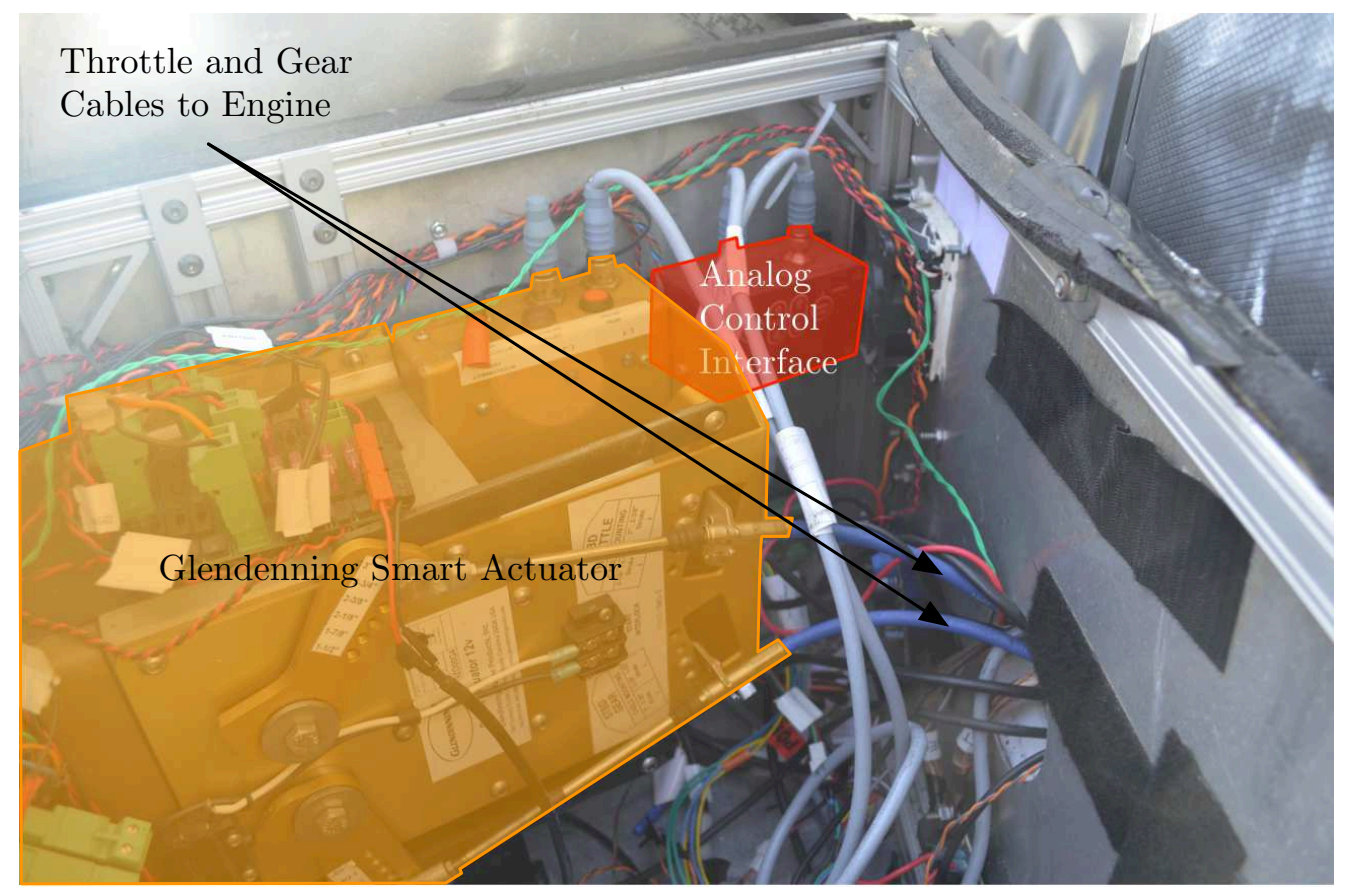

Figure 3.7: The Smart Actuator and Analog Control Interface to control throttle

range algorithms are used in the ECU and are consequently not discussed in this description. The ECU calculates distances up to $100 \mathrm{~m}$ at a resolution of $1 \mathrm{~cm}$ and distances above $100 \mathrm{~m}$ at a resolution of 10 $\mathrm{cm}$. The ECU's parameters can be adjusted and calculations measured from either a CAN bus or ethernet connection while an RS232 port can provide synchronization between multiple devices. Due to its ease of implementation, the ethernet connection on the ECU is used on the Ribcraft USV. The laser line scanner is shown in Figure 3.9. Combining range and relative angle measurements with GPS position and heading gives absolute position of obstacles in space.

The laser line scanner is nominally pointed towards the nose of the vessel, but additional fine tuning of its orientation is required to avoid errant laser scans of water or sky replacing useful scans of the horizon. A DirectedPerception PTU-D100 gimbal pitches and rolls the laser line scanner in concert with orientation measurements from a Microstrain 3DM-GX1 AHRS, which is mounted with the laser line scanner, to im- 


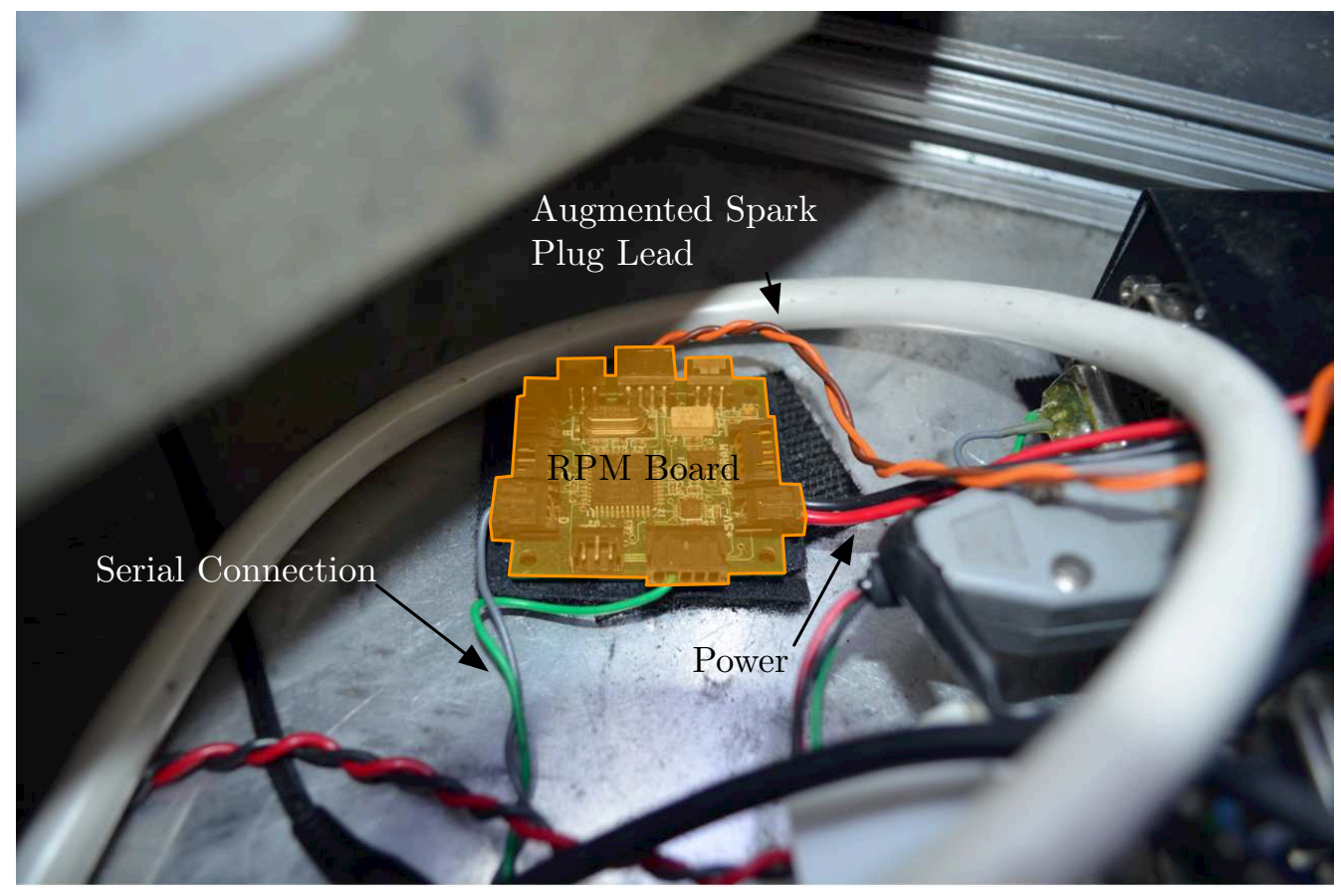

Figure 3.8: The engine RPM sensor

prove shoreline detection. This gimbal uses two high strength stepper motors in order to rotate up to 15 pounds of payload along two axes. Open loop serial commands via RS232 or RS485 cables yield gimbal movement to desired positions at user defined rates. On initialization the gimbal pans and tilts through its entire range of motion to calibrate the device, giving reliable convergence to desired orientations as long as the stepper motors do not slip. Closed loop control using pitch and roll measurements from the AHRS avoids issues of stepper motor slippage while enhancing shore line scans by keeping the laser scanner level in pitch and roll relative to the horizon during aggressive maneuvering induces large pitch and roll angles.

The Microstrain 3DM-GX1 fuses measurements from 3 orthogonal on-board magnetometers, which read Earth's magnetic field direction, and 3 orthogonal linear accelerometers, which read acceleration due to vehicle motion and gravity, to find a moving orientation frame, which can be interpreted as Euler angles, quaternions, or a rotation cosine matrix. Iron calibration and temperature correction improve accuracy of the magnetometers and accelerometers respectively. Angular rate gyroscopes, which are predominately free 


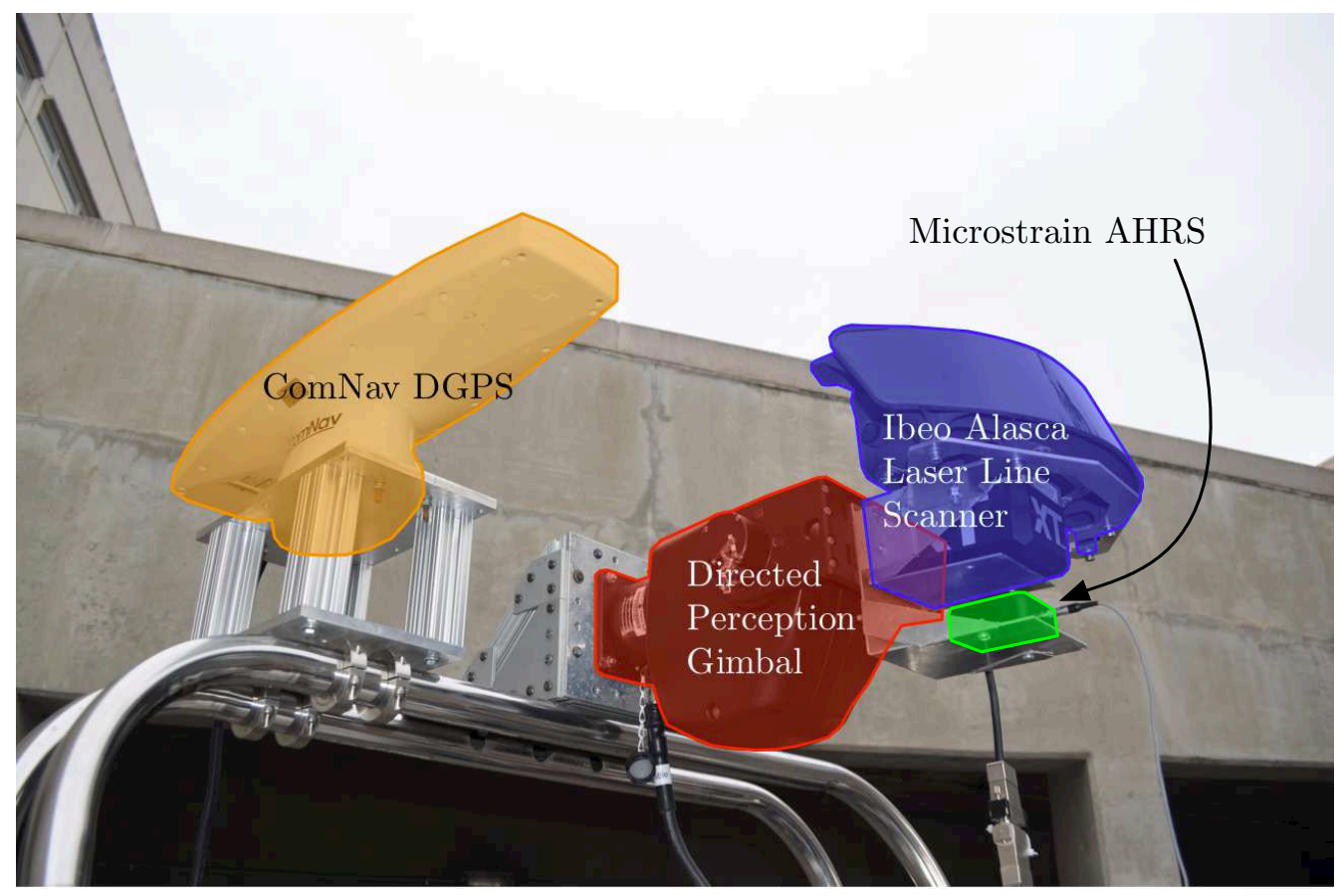

Figure 3.9: The DGPS, gimbal, laser line scanner, and AHRS

of drift, give angular rate information without the need of the other sensors. In addition to keeping the laser line scanner level, the gimbal and AHRS setup can be commanded to give small amplitude sinusoids in pitch relative to the horizon in order to identify obstacles both near and far, generating better data. The gimbal and AHRS are shown in Figure 3.9. With the description of the hardware complete, discussion of the software suite, which measures the sensors and animates the actuators of the Ribcraft USV, completes the test vehicle discussion.

\subsection{The Autonomy Software}

Closed loop control algorithms must combine reading of sensor data and actuation of inputs to yield desired performance. Logging of both sensor data and actuator positions is essential to identifying vessel dynamics, debugging controller software, and displaying results for validation and publication. Organizing these mul- 
tiple input and output data streams in a coherent manner requires cleverly designed software structure that is general enough to allow for a multitude of sensor and actuator combinations and configurations.

The software, written in $\mathrm{C}++$, borrows heavily from an in-house library developed by Brian McCarter dubbed the Autonomous Application Framework (AAF). This library defines a fundamental base class called "Managed," which contains elemental features that all components of devices, sensors, commands, and controllers share. This class is derived from the "Echelon" class that provides a program wide event framework. The main program begins by calling "USV" and "Control" functions, which use the "Factory" class templated on "Managed" transport, device, command, and controller objects, which yields transport, device, command, and controller "factories". These factories" "register" functions instantiate all constituent objects while imbuing them with a shared pointer and mapping, allowing for appropriate object calling and destruction. A thread is created when any "Managed" object is instantiated, allowing for all "Managed" objects to run concurrently. With all of the objects instantiated, the software is now running, reading sensors, and ready to execute commands by commanding devices. However, knowledge of the intricacies of instantiation, software/device interfacing, and inter-process and outside-software communication are still required to fully understand how the software operates.

The USV software reads XML configuration files upon initialization of the code by using the Boost "Property Tree" library wrapped by the "Config" class. Registering each constituent "Managed" object, while giving the compiler knowledge of all available objects, does not instantiate the object. Instantiation occurs when the register command is processed, causing a query to the appropriate XML file that indicates whether the "Managed" object will be instantiated. Once instantiated and a thread created, the object's own "Config" function reads additional configuration information, which might include things like serial port addresses for sensors and devices, controller gains and filter cutoff frequencies for control algorithms, and vessel dynamic parameters for the simulator. These XML files are read only once, only during initial software start up, so for any edit to the configuration files to take effect, the software must be stopped and restarted. Using XML configuration files allows entire chunks of device and control code to be included or omitted dynamically and enables gain adjustments without the need for lengthy recompilation of the code.

Interfacing between hardware components and the software is accomplished mostly through serial com- 
munication, though the laser line scanner requires TCP/IP communication through an ethernet connection. Wrapping both the TCP/IP and serial communication functions found in the Boost "ASIO" library with the "Transport" class allows for seamless use of input/output functionality in the AAF library. Asynchronous reading and writing is used in place of blocking functions to prevent portions of code from hanging for longer than a user specified timeout, which is defined in the appropriate "Config" function. Writing to serial ports is the primary vehicle for commanding the digital potentiometer and motor controller and querying rudder position information and orientation from the motor controller and AHRS. Note that the DGPS and RPM sensor constantly send information at a set rate, which only requires asynchronous reading at a fast enough rate to prevent the buffer from filling.

Though communication between software and hardware has been discussed, an efficient way to communicate common data between multiple software processes and independent programs is essential to simplify implementation of closed loop control laws and path planning algorithms. The Lightweight Communications and Marshalling (LCM) is a set of tools and libraries originally developed for the MIT DARPA Urban Challenge Team used to allow multiple devices and programs to communicate quickly in a high bandwidth environment. This communication method centers around UDP multicast, which requires no centralized data server and yields very fast information transfer at the cost potential packet loss and ordering mismatch. Aside from simplifying communication within a single program the benefits of using LCM also includes the ability to communicate between different programs written using different languages $(\mathrm{C}, \mathrm{C}++$, Java, Python, MATLAB, and C\# are supported). This cross-program capability allows the same data to be used with both a script language, used in preliminary algorithm development and validation, and a compiled language, used to implement said algorithms. Also, all LCM data is cross-platform and can be used simultaneously with 32 bit and 64 bit hardware architecture, vastly simplifying a potentially complex task. The "LCM" class in AAF acts as a wrapper integrating the basic available functionality of the LCM library.

Note that software communication for some of the core functionality does not use this broadcasting protocol, instead opting to simply use mutually exclusive read/write logic and using the object's "get" functions to transfer data between objects, however, this method becomes convoluted when multiple objects require data from a single thread. Each of the objects needing data must individually lock data for reading and 
writing, requiring a complex series of actions for intuitively simple functionality. To make matters worse, debugging random runtime errors like segmentation faults and other issues due to nuanced mistakes in writing this communication method requires a considerable additional time investment over standard debugging practices.

Logging information from sensors and inputs, control subsystem states, and error messages is essential for debugging control law behavior and complex software actions. With a bug free software framework and control laws, sensor and input logs provide validation information that can be displayed in plots showing controller performance. The AAF library provides a wrapper for the Apache log $4 \mathrm{cxx}$ logging framework to give logging functionality. Logging of the sensors, inputs, and control subsystems only requires a file to be created and a header printed in the initialization functions of devices and control laws, followed by function calls that print fresh information as it arrives. However, logging of software behavior such as thread instantiation and communication requires logging to be prioritized as trace, debug, info, warn, error, and fatal allowing messages to be enabled and disabled appropriately. Logging of these messages is saved to multiple locations by using appenders allowing messages to be segregated based on level while also being collected into a single file. Note that the logging discussed thus far involves printing data to multiple files that can be read by simple text editors, however, the LCM protocol also includes a logging functionality that broadcasts UDP data exactly as it was observed in the field. These saved files can be played like a record in real time or faster or slower than reality. The ability to recreate actual UDP broadcasts allows for software debugging in the lab that exactly recreates the conditions in the field.

A Cascading controller structure simplifies how multiple simple controllers interact. Additionally, complicated control algorithms with a cascaded structure can be divided into elemental sub-controllers by using the cascaded structure. First, an input is sent to the control structure, which then trickles down a predefined input hierarchy until an enabled controller that accepts the given input is encountered. The input is not acted upon and "drops out of the bottom" of the hierarchy if no appropriate controller is enabled. The controller that accepts the given input computes an output that either directly commands an actuator or becomes another input, which again trickles down the input hierarchy. This process is repeated until the final input drops out of the bottom of the hierarchy or a command is sent to an actuator. The controller with the highest 
order in the hierarchy is used in cases where multiple control elements contain the same input. A control input could feasibly be used on one control element, which outputs the same type of control input that is acted upon be another controller lower in the hierarchy. It will be seen in the control section that the PD cascade trajectory tracking controller takes advantage of this hierarchical structure. However, this control structure is largely left unused for more advanced nonlinear controllers.

To expedite the implementation of advanced controllers, as much debugging as possible must be relegated to simulations in the laboratory environment to avoid time intensive tests in the field. The AAF library contains a simulator class, which provides the framework necessary to develop simulations of actual vessels. A "run" function takes states and inputs from the previous time step and propagates them in time using a prescribed model structure with parameters populated from an XML file read in a config function (like all other managed classes in AAF). The states propagated forward are sent to functions that emulate AHRS and DGPS measurements, which are broadcast using the appropriate LCM device channels. Control laws see these LCM broadcasts as if they were actual devices that react to actuator adjustments like the actual vessel as long as the models accurately estimate vessel dynamics. Bug free control laws developed in a scripting language like MATLAB or Simulink can be compared to the $\mathrm{C}++$ control simulations until the $\mathrm{C}++$ control law is also bug free. With the implemented $\mathrm{C}++$ control laws completely debugged, only control gain adjustments in the field, used to account for discrepancies in the simulated and actual vessel, are required.

Installation and development of the autonomy package was a team effort. However, the explicit contributions the author has made include:

- Installation of the manual e-stop and throttle lever

- Installation and calibration of the Smart Actuator system

- Development of the PIC digital engine RPM measuring board

- Augmentation of existing RPM code to read output of PIC chip

- Development of the Op Amp board enhancing rudder actuation and position resolution

- Augmentation of rudder software allowing for correct measurement of rudder position 
- Implementation of up to third order filters giving smoothed signals their derivatives

- Adjustment of turn rate, RPM, and speed PID controllers using a priori steady state relationships that allows for additional feedforward inputs

- Implementation of a cascaded PD trajectory tracking controller utilizing aforementioned PID controllers

- Implementation of a backstepping control algorithm

- Inclusion of Ribcraft USV motion models into the simulator class

The Ribcraft USV with the complete autonomy package installed is shown in Figure 3.10.

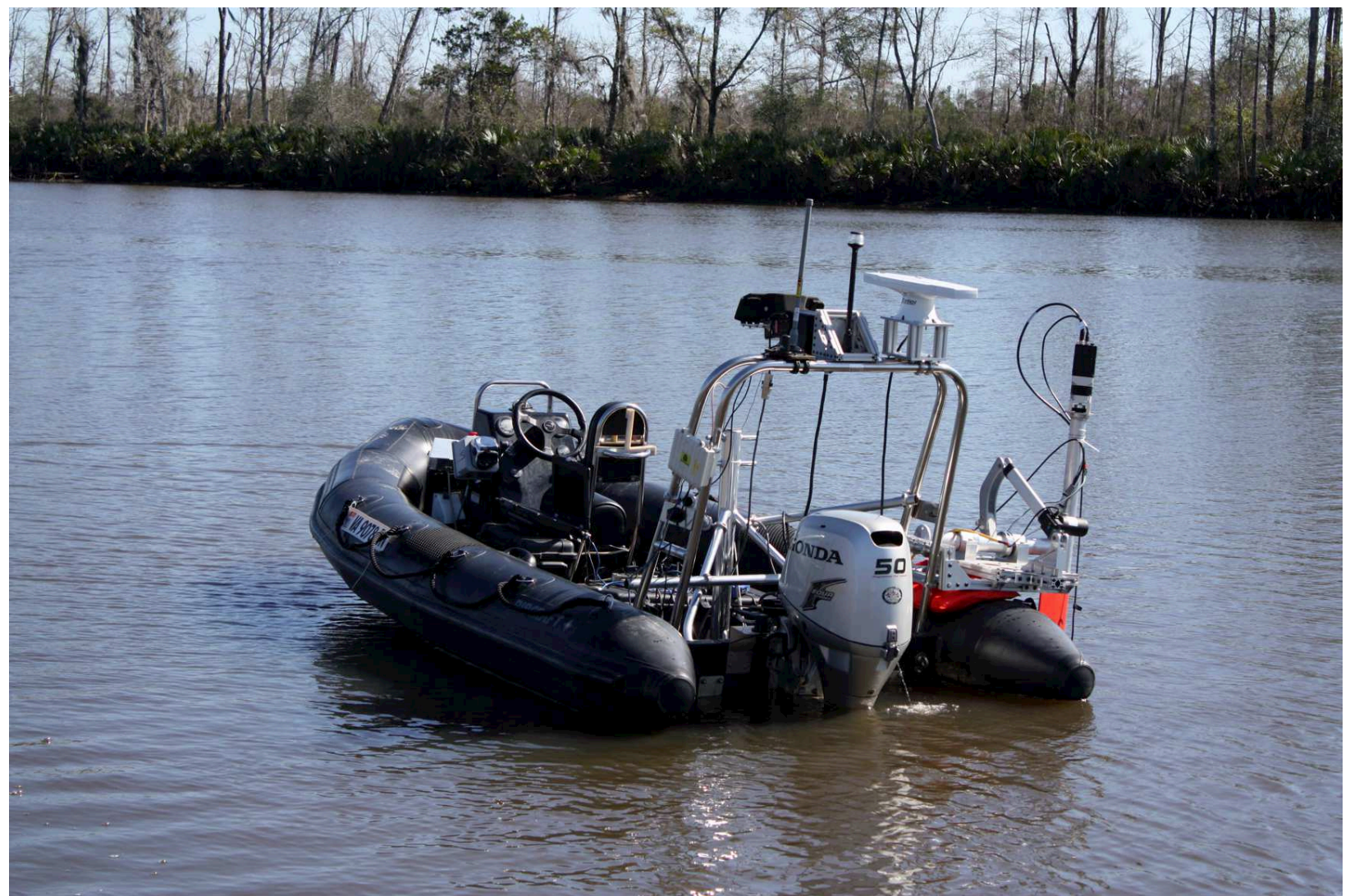

Figure 3.10: The fully autonomous Ribcraft USV 


\title{
CHAPTER
}

\author{
4
}

\section{USV DYNAMIC MODELING}

Having developed a nonlinear, physics-based model, one may consider this and/or reduced complexity models as candidate models for motion planning and control design. Conventional analysis of the six DOF motion of a rigid vessel is decoupled into a "seakeeping" model capturing vessel behavior in waves and a "maneuvering" model describing motion in the horizontal plane. The three DOF maneuvering mode ignores roll, pitch, and heave dynamics. The water is assumed to be calm and, for initial modeling and identification purposes, currents are neglected. The effects of currents and flow gradients on marine vessel dynamics have been carefully considered in [Woolsey, 2011, Thomasson and Woolsey, 2012]; it is straight forward to incorporate these effects into the USV dynamic model, if necessary.

Hydrodynamicists characterize surface vessel performance in terms of the Froude number Fr $=U / \sqrt{L g}$, where $U$ is the operating speed, $L$ is the length of the submerged portion of the hull, and $g$ is the acceleration due to gravity. As Faltinsen [Faltinsen, 2005] explains, the weight of a vessel operating with a Froude 
number less than about 0.4 to 0.5 is almost completely supported by the hydrostatic force of buoyancy, a condition called displacement mode. At the other extreme, in planing mode, the weight of a vessel operating with a Froude number greater than about 1.0 to 1.2 is almost completely supported by a hydrodynamic force that scales roughly with the square of speed. For intermediate values of the Froude number, both the hydrostatic and hydrodynamic force contribute significantly in balancing the vessel's weight, a condition called semi-displacement mode. Recall that the Ribcraft USV is designed as a planing vessel, but it routinely operates in each of the three modes. A successful modeling effort will adequately capture the variations in vehicle performance over the full range of achievable Froude numbers.

\subsection{Kinematics}

The USV is modeled as a rigid hull with an articulated outboard motor. Define a body-fixed reference frame whose $x$-axis (denoted by the unit vector $\boldsymbol{b}_{x}$ ) points forward along the longitudinal axis of the boat, whose $y$-axis (denoted by the unit vector $\boldsymbol{b}_{y}$ ) points to starboard, and whose $z$-axis (denoted $\boldsymbol{b}_{z}=\boldsymbol{b}_{x} \times$ $\boldsymbol{b}_{y}$ ) points downward, completing the orthonormal triad. We assume that the USV is symmetric about its longitudinal plane and that the body reference frame is chosen such that the inertia tensor is diagonal: $\boldsymbol{I}=\operatorname{diag}\left(I_{x x}, I_{y y}, I_{z z}\right)$.

Suppose that the vector $\boldsymbol{x}$ denotes the position of the body coordinate origin with respect to some inertially fixed reference frame, denoted by the unit vectors $\boldsymbol{i}_{x}, \boldsymbol{i}_{y}$, and $\boldsymbol{i}_{z}$. Let $\boldsymbol{R}$ denote the proper rotation matrix which maps free vectors from the body frame to the inertial frame. Let the body vector $\boldsymbol{v}=(u, v, w)^{T}$ represent the translational velocity of the body reference frame with respect to the inertial reference frame. Similarly, let $\boldsymbol{\omega}=(p, q, r)^{T}$ represent the body angular rate relative to the inertial reference frame shown in Figure 4.1.

The rigid body kinematic equations are

$$
\begin{aligned}
\dot{x} & =\boldsymbol{R} \boldsymbol{v} \\
\dot{\boldsymbol{R}} & =\boldsymbol{R} \hat{\boldsymbol{\omega}}
\end{aligned}
$$




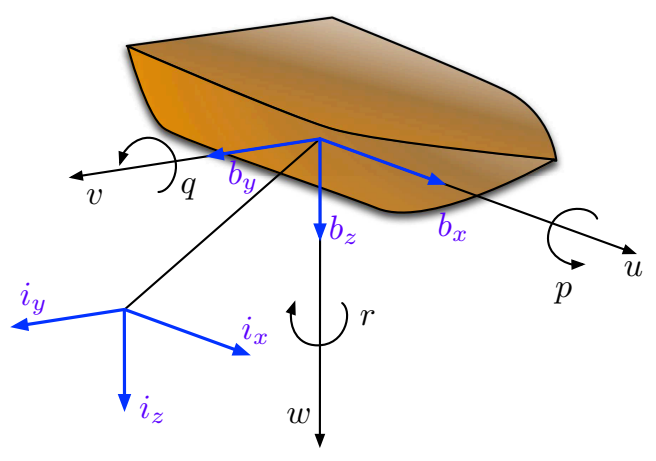

\section{Figure 4.1: Body fixed 6 DOF reference frame}

where the notation $\hat{\imath}$ denotes the $3 \times 3$ skew-symmetric matrix satisfying $\hat{\boldsymbol{a}} \boldsymbol{b}=\boldsymbol{a} \times \boldsymbol{b}$ for vectors $\boldsymbol{a}$ and $\boldsymbol{b}$. These equations relate the body's translational and rotational velocity to the rate of change of position and attitude. The matrix $\boldsymbol{R}$ is typically expressed in coordinates, using the conventional Euler angles $\phi, \theta$, and $\psi$, for example. In this case, the matrix differential equation (4.2) is replaced by three equations relating $\dot{\phi}$, $\dot{\theta}$, and $\dot{\psi}$ to $\omega$. Specifically,

$$
\dot{\boldsymbol{\eta}}=\boldsymbol{J}(\boldsymbol{\eta}) \boldsymbol{\nu}
$$

where the states of this system are

$$
\begin{aligned}
& \boldsymbol{\nu}=\left[\begin{array}{llllll}
u & v & w & p & q & r
\end{array}\right]^{T} \\
& \boldsymbol{\eta}=\left[\begin{array}{llllll}
x & y & z & \phi & \theta & \psi
\end{array}\right]^{T}
\end{aligned}
$$


and

$$
\begin{aligned}
& \boldsymbol{J}(\boldsymbol{\eta})=\left(\begin{array}{cccc}
\cos \psi \cos \theta & -\sin \psi \cos \phi+\cos \psi \sin \theta \sin \phi & \sin \phi \sin \psi+\cos \psi \cos \phi \sin \theta & 0 \\
\sin \psi \cos \theta & \cos \psi \cos \phi+\sin \phi \sin \theta \sin \psi & -\cos \psi \sin \phi+\sin \theta \sin \psi \cos \phi & 0 \\
-\sin \theta & \cos \theta \sin \phi & \cos \theta \cos \phi & 0 \\
0 & 0 & 0 & 1 \\
0 & 0 & 0 & 0 \\
0 & 0 & 0 & 0
\end{array}\right. \\
& \left.\begin{array}{cc}
0 & 0 \\
0 & 0 \\
0 & 0 \\
\operatorname{m} \phi \tan \theta & \cos \phi \tan \theta \\
\cos \phi & -\sin \phi \\
\frac{\sin \phi}{\cos \theta} & \frac{\cos \phi}{\cos \theta}
\end{array}\right)
\end{aligned}
$$

\subsection{Nonlinear 6 DOF Dynamics}

The dynamics of a boat can be described as

$$
M \dot{\nu}+C(\nu) \nu+D(\nu) \nu+g(\eta)=\tau
$$

where $M$ is the mass matrix, $C$ is the Coriolis and centripetal matrix, $\boldsymbol{D}$ is the damping matrix, $\boldsymbol{g}$ is a vector of restoring forces and moments due to the interaction between buoyancy and gravity, and $\tau$ is a vector of control forces and moments.

Effects of buoyancy's interaction with gravity, manifested in $\boldsymbol{g}(\boldsymbol{\eta})$, give the metacentric stability proper- 
ties of a ship. Simplifying to bodies with symmetry in the xz-plane gives

$$
\boldsymbol{g}(\boldsymbol{\eta})=-\left(\begin{array}{c}
-\rho g \int_{0}^{z} A_{w p}(\tau) d \tau \sin \theta \\
\rho g \int_{0}^{z} A_{w p}(\tau) d \tau \cos \theta \sin \phi \\
\rho g \int_{0}^{z} A_{w p}(\tau) d \tau \cos \theta \cos \phi \\
\rho g \nabla \overline{G M}_{T} \sin \phi \cos \theta \cos \phi \\
\rho g \nabla \overline{G M}_{L} \sin \theta \cos \theta \cos \phi \\
\rho g \nabla\left(-\overline{G M}_{L} \cos \theta+\overline{G M}_{T}\right) \sin \phi \sin \theta
\end{array}\right)
$$

where $\rho$ is water density, $g$ is gravity, $z$ is the depth at the lowest point of the vehicle, $\nabla$ is displaced water volume, $A_{w p}$ is the water plane area, $\overline{G M}_{T}$ is the transverse metacentric height, and $\overline{G M}_{L}$ is the longitudinal metacentric height.

The mass matrix $M$ and Coriolis and centripetal matrix $C$ can be decomposed to

$$
\boldsymbol{M}=\boldsymbol{M}_{A}+\boldsymbol{M}_{R B} \quad \text { and } \quad \boldsymbol{C}(\boldsymbol{\nu})=\boldsymbol{C}_{A}(\boldsymbol{\nu})+\boldsymbol{C}_{R B}(\boldsymbol{\nu})
$$

where the subscript "RB" denotes rigid body contributions and the subscript "A" represents added mass and inertia.

Looking exclusively at the rigid body terms we have the symmetric positive definite mass matrix

$$
\boldsymbol{M}_{R B}=\left(\begin{array}{cccccc}
m & 0 & 0 & 0 & m z_{G} & -m y_{G} \\
0 & m & 0 & -m z_{G} & 0 & m x_{G} \\
0 & 0 & m & m y_{G} & -m x_{G} & 0 \\
0 & -m z_{G} & m y_{G} & I_{x x} & -I_{x y} & -I_{x z} \\
m z_{G} & 0 & -m x_{G} & -I_{y x} & I_{y y} & -I_{y z} \\
-m y_{G} & m x_{G} & 0 & -I_{z x} & -I_{z y} & I_{z z}
\end{array}\right)
$$

where $m$ is the total mass, $\left(\begin{array}{ccc}x_{G} & y_{G} & z_{G}\end{array}\right)^{T}$ is the vector between the center of gravity and vehicle origin, 
and

$$
\boldsymbol{I}=\left(\begin{array}{ccc}
I_{x x} & -I_{x y} & -I_{x z} \\
-I_{y x} & I_{y y} & -I_{y z} \\
-I_{z x} & -I_{z y} & I_{z z}
\end{array}\right)
$$

is the inertial tensor that accounts for lack of symmetry with off-diagonal terms. Using the parallel axis theorem gives the terms containing the mass variable combined with elements of the center of gravity vector, which adds to the rotational inertia. The corresponding rigid body Coriolis and centripetal matrix is

$$
\begin{gathered}
\boldsymbol{C}(\boldsymbol{\nu})=\left(\begin{array}{cccc}
0 & 0 & 0 & m\left(y_{G} q+z_{G} r\right) \\
0 & 0 & 0 & -m\left(y_{G} p+w\right) \\
0 & 0 & 0 & -m\left(z_{G} p-v\right) \\
-m\left(y_{G} q+z_{G} r\right) & m\left(y_{G} p+w\right) & m\left(z_{G} p-v\right) & 0 \\
m\left(x_{G} q-w\right) & -m\left(z_{G} r+x_{G} p\right) & m\left(z_{G} q+u\right) & I_{y z} q+I_{x z} p-I_{z z} r \\
m\left(x_{G} r+v\right) & m\left(y_{G} r-u\right) & -m\left(x_{G} p+y_{G} q\right) & -I_{y x z} r-I_{x y} p+I_{y y} q \\
-m\left(x_{G} q-w\right) & -m\left(x_{G} r+v\right) \\
m\left(z_{G} r+x_{G} p\right) & -m\left(y_{G} r-u\right) & \\
-m\left(z_{G} q+u\right) & m\left(x_{G} p+y_{G} q\right) \\
-I_{y z} q-I_{x z} p+I_{z z} r & I_{y z} r+I_{x y} p-I_{y y} q \\
0 & -I_{x z} r-I_{x y} q+I_{x x} p \\
I_{x z} r+I_{x y} q-I_{x x} p & 0
\end{array}\right.
\end{gathered}
$$

The vessel accelerates nearby fluid around its body during motion, effectively adding inertia to the system, which is accounted for by including added mass terms. All vehicles moving in a fluid (including heavierthan-air aircraft) have added mass, but its effects become more pronounced when the density of the fluid 
approaches as the density of the vehicle. The added mass matrix is defined as

$$
\boldsymbol{M}_{A}=-\left(\begin{array}{cccccc}
X_{\dot{u}} & X_{\dot{v}} & X_{\dot{w}} & X_{\dot{p}} & X_{\dot{q}} & X_{\dot{r}} \\
Y_{\dot{u}} & Y_{\dot{v}} & Y_{\dot{w}} & Y_{\dot{p}} & Y_{\dot{q}} & Y_{\dot{r}} \\
Z_{\dot{u}} & Z_{\dot{v}} & Z_{\dot{w}} & Z_{\dot{p}} & Z_{\dot{q}} & Z_{\dot{r}} \\
K_{\dot{u}} & K_{\dot{v}} & K_{\dot{w}} & K_{\dot{p}} & K_{\dot{q}} & K_{\dot{r}} \\
M_{\dot{u}} & M_{\dot{v}} & M_{\dot{w}} & M_{\dot{p}} & M_{\dot{q}} & M_{\dot{r}} \\
N_{\dot{u}} & N_{\dot{v}} & N_{\dot{w}} & N_{\dot{p}} & N_{\dot{q}} & N_{\dot{r}}
\end{array}\right)
$$

Knowing the added mass matrix of a system allows for the immediate derivation of the corresponding added mass Coriolis and centripetal matrix. Using the equation for fluid kinetic energy

$$
T_{A}=\frac{1}{2} \nu^{T} M_{A} \boldsymbol{\nu}
$$

with Kirchhoff's equations for a rigid body moving through an incompressible fluid gives

$$
\begin{aligned}
& \frac{d}{d t} \frac{\partial T_{A}}{\partial u}=r \frac{\partial T_{A}}{\partial v}-q \frac{\partial T_{A}}{\partial w}+X_{A} \\
& \frac{d}{d t} \frac{\partial T_{A}}{\partial v}=-p \frac{\partial T_{A}}{\partial w}-r \frac{\partial T_{A}}{\partial u}-Y_{A} \\
& \frac{d}{d t} \frac{\partial T_{A}}{\partial r}=q \frac{\partial T_{A}}{\partial u}-p \frac{\partial T}{\partial v}+Z_{A} \\
& \frac{d}{d t} \frac{\partial T_{A}}{\partial u}=w \frac{\partial T_{A}}{\partial v}-v \frac{\partial T_{A}}{\partial w}+r \frac{\partial T_{A}}{\partial q}-q \frac{\partial T_{A}}{\partial r}-K_{A} \\
& \frac{d}{d t} \frac{\partial T_{A}}{\partial v}=u \frac{\partial T_{A}}{\partial w}-w \frac{\partial T_{A}}{\partial r}-r \frac{\partial T_{A}}{\partial p}-M_{A} \\
& \frac{d}{d t} \frac{\partial T_{A}}{\partial r}=v \frac{\partial T_{A}}{\partial u}-u \frac{\partial T_{A}}{\partial v}+q \frac{\partial T_{A}}{\partial p}-p \frac{\partial T_{A}}{\partial q}-N_{A}
\end{aligned}
$$


Extracting the added mass Coriolis and centripetal matrix we have

$$
\boldsymbol{C}(\boldsymbol{\nu})=\left(\begin{array}{cccccc}
0 & 0 & 0 & 0 & -C_{3} & C_{2} \\
0 & 0 & 0 & C_{3} & 0 & -C_{1} \\
0 & 0 & 0 & -C_{2} & C_{1} & 0 \\
0 & -C_{3} & C_{2} & 0 & -C_{6} & C_{5} \\
C_{3} & 0 & -C_{1} & C_{6} & 0 & -C_{4} \\
-C_{2} & C_{2} & 0 & -C_{5} & C_{4} & 0
\end{array}\right)
$$

where

$$
\begin{aligned}
& C_{1}=X_{\dot{u}} u+X_{\dot{v}} v+X_{\dot{w}} w+X_{\dot{p}} p+X_{\dot{q}} q+X_{\dot{r}} r \\
& C_{2}=X_{\dot{v}} u+Y_{\dot{v}} v+Y_{\dot{w}} w+Y_{\dot{p}} p+Y_{\dot{q}} q+Y_{\dot{r}} r \\
& C_{3}=X_{\dot{w}} u+Y_{\dot{w}} v+Z_{\dot{w}} w+Z_{\dot{p}} p+Z_{\dot{q}} q+Z_{\dot{r}} r \\
& C_{4}=X_{\dot{p}} u+Y_{\dot{p}} v+Z_{\dot{p}} w+K_{\dot{p}} p+K_{\dot{q}} q+K_{\dot{r}} r \\
& C_{5}=X_{\dot{q}} u+Y_{\dot{q}} v+Z_{\dot{q}} w+K_{\dot{q}} p+M_{\dot{q}} q+M_{\dot{r}} r \\
& C_{6}=X_{\dot{r}} u+Y_{\dot{r}} v+Z_{\dot{r}} w+K_{\dot{r}} p+M_{\dot{r}} q+N_{\dot{r}} r
\end{aligned}
$$

The damping matrix is composed of a linear damping matrix and quadratic damping matrix such that

$$
\boldsymbol{D}=\boldsymbol{D}_{l}+\boldsymbol{D}_{q}
$$


The linear damping matrix arrises due to laminar friction on the body of the boat and takes the form

$$
\boldsymbol{D}_{l}=-\left(\begin{array}{cccccc}
X_{u} & X_{v} & X_{w} & X_{p} & X_{q} & X_{r} \\
Y_{u} & Y_{v} & Y_{w} & Y_{p} & Y_{q} & Y_{r} \\
Z_{u} & Z_{v} & Z_{w} & Z_{p} & Z_{q} & Z_{r} \\
K_{u} & K_{v} & K_{w} & K_{p} & K_{q} & K_{r} \\
M_{u} & M_{v} & M_{w} & M_{p} & M_{q} & M_{r} \\
N_{u} & N_{v} & N_{w} & N_{p} & N_{q} & N_{r}
\end{array}\right)
$$

The quadratic damping matrix comes about due to pressure drag off of the hull of the boat and is shown as

$$
\boldsymbol{D}_{q}(\nu)=\left(\begin{array}{cccccc}
X_{u|u|}|u| & X_{v|v|}|v| & X_{w|w|}|w| & X_{p|p|}|p| & X_{q|q|} & X_{r|r|}|r| \\
Y_{u|u|}|u| & Y_{v|v|}|v| & Y_{w|w|}|w| & Y_{p|p|}|p| & Y_{q|q|}|q| & Y_{r|r|}|r| \\
Z_{u|u|}|u| & Z_{v|v|}|v| & Z_{w|w|}|w| & Z_{p|p|}|p| & Z_{q|q|}|q| & Z_{r|r|}|r| \\
K_{u|u|}|u| & K_{v|v|}|v| & K_{w|w|}|w| & K_{p|p|}|p| & K_{q|q|}|q| & K_{r|r|}|r| \\
M_{u|u|}|u| & M_{v|v|}|v| & M_{w|w|}|w| & M_{p|p|}|p| & M_{q|q|}|q| & M_{r|r|}|r| \\
N_{u|u|}|u| & N_{v|v|}|v| & N_{w|w|}|w| & N_{p|p|}|p| & N_{q|q|}|q| & N_{r|r|}|r|
\end{array}\right)
$$

Applying assumptions of port/starboard symmetry considerably simplifies the added mass matrix giving

$$
\boldsymbol{M}_{A}=-\left(\begin{array}{cccccc}
X_{\dot{u}} & 0 & X_{\dot{w}} & 0 & X_{\dot{q}} & 0 \\
0 & Y_{\dot{v}} & 0 & Y_{\dot{p}} & 0 & Y_{\dot{r}} \\
Z_{\dot{u}} & 0 & Z_{\dot{w}} & 0 & Z_{\dot{q}} & 0 \\
0 & K_{\dot{v}} & 0 & K_{\dot{p}} & 0 & K_{\dot{r}} \\
M_{\dot{u}} & 0 & M_{\dot{w}} & 0 & M_{\dot{q}} & 0 \\
0 & N_{\dot{v}} & 0 & N_{\dot{p}} & 0 & N_{\dot{r}}
\end{array}\right)
$$

In its current state the added mass matrix is positive definite but not symmetric. In the presence of waves and forward vessel motion this matrix will stay asymmetric, but for slow speeds on inland bodies of water 
the waves can be assumed to be small enough to allow for symmetry [Fossen, 1995]. This gives

$$
\boldsymbol{M}_{A}=-\left(\begin{array}{cccccc}
X_{\dot{u}} & 0 & Z_{\dot{u}} & 0 & M_{\dot{u}} & 0 \\
0 & Y_{\dot{v}} & 0 & K_{\dot{v}} & 0 & N_{\dot{v}} \\
Z_{\dot{u}} & 0 & Z_{\dot{w}} & 0 & M_{\dot{w}} & 0 \\
0 & K_{\dot{v}} & 0 & K_{\dot{p}} & 0 & N_{\dot{p}} \\
M_{\dot{u}} & 0 & M_{\dot{w}} & 0 & M_{\dot{q}} & 0 \\
0 & N_{\dot{v}} & 0 & N_{\dot{p}} & 0 & N_{\dot{r}}
\end{array}\right)
$$

However, the vessel will be traveling at a nontrivial forward speed requiring (4.2), which becomes

$$
\boldsymbol{M}=\left(\begin{array}{cccccc}
m-X_{\dot{u}} & 0 & -X_{\dot{w}} & 0 & m z_{G}-X_{\dot{q}} & 0 \\
0 & m-Y_{\dot{v}} & 0 & -m z_{G}-Y_{\dot{p}} & 0 & m x_{G}-Y_{\dot{r}} \\
-Z_{\dot{u}} & 0 & m-Z_{\dot{w}} & m y_{G} & -m x_{G}-Z_{\dot{q}} & 0 \\
0 & -m z_{G}-K_{\dot{v}} & 0 & I_{x}-K_{\dot{p}} & 0 & -I_{x z}-K_{\dot{r}} \\
m z_{G}-M_{\dot{u}} & 0 & -m x_{G}-M_{\dot{w}} & 0 & I_{y}-M_{\dot{q}} & 0 \\
0 & m x_{G}-N_{\dot{v}} & 0 & -I_{z x}-N_{\dot{p}} & 0 & I_{z}-N_{\dot{r}}
\end{array}\right)
$$

when combined with the rigid body mass matrix. Making the assumption that the center of rotation matches the center of gravity in the pitch and roll axes allows the mass matrix to become

$$
M=\left(\begin{array}{cccccc}
m-X_{\dot{u}} & 0 & -X_{\dot{w}} & 0 & -X_{\dot{q}} & 0 \\
0 & m-Y_{\dot{v}} & 0 & -Y_{\dot{p}} & 0 & m x_{G}-Y_{\dot{r}} \\
-Z_{\dot{u}} & 0 & m-Z_{\dot{w}} & 0 & -m x_{G}-Z_{\dot{q}} & 0 \\
0 & -K_{\dot{v}} & 0 & I_{x}-K_{\dot{p}} & 0 & -I_{x z}-K_{\dot{r}} \\
-M_{\dot{u}} & 0 & -m x_{G}-M_{\dot{w}} & 0 & I_{y}-M_{\dot{q}} & 0 \\
0 & m x_{G}-N_{\dot{v}} & 0 & -I_{z x}-N_{\dot{p}} & 0 & I_{z}-N_{\dot{r}}
\end{array}\right)
$$


subsequently yielding the Coriolis and centripetal matrix

$$
\begin{aligned}
& \boldsymbol{C}(\boldsymbol{\nu})=\left(\begin{array}{ccc}
0 & 0 & 0 \\
0 & 0 & 0 \\
0 & 0 & 0 \\
-m\left(y_{G} q+z_{G} r\right) & m\left(y_{G} p+w\right)-C_{3_{\text {trunc }}} & m\left(z_{G} p-v\right)+C_{2_{\text {trunc }}} \\
m\left(x_{G} q-w\right)+C_{3_{\text {trunc }}} & -m\left(z_{G} r+x_{G} p\right) & m\left(z_{G} q+u\right)-C_{1_{\text {trunc }}} \\
m\left(x_{G} r+v\right)-C_{2_{\text {trunc }}} & m\left(y_{G} r-u\right)+C_{2_{\text {trunc }}} & -m\left(x_{G} p+y_{G} q\right)
\end{array}\right. \\
& m\left(y_{G} q+z_{G} r\right) \quad-m\left(x_{G} q-w\right)-C_{3_{\text {trunc }}} \quad-m\left(x_{G} r+v\right)+C_{2_{\text {trunc }}} \\
& -m\left(y_{G} p+w\right)+C_{3_{\text {trunc }}} \quad m\left(z_{G} r+x_{G} p\right) \quad-m\left(y_{G} r-u\right)-C_{1_{\text {trunc }}} \\
& -m\left(z_{G} p-v\right)-C_{2_{\text {trunc }}} \quad-m\left(z_{G} q+u\right)+C_{1_{\text {trunc }}} \quad m\left(x_{G} p+y_{G} q\right) \\
& 0 \quad-I_{y z} q-I_{x z} p+I_{z z} r-C_{6 \text { trunc }} \quad I_{y z} r+I_{x y} p-I_{y y} q+C_{5 \text { trunc }} \\
& I_{y z} q+I_{x z} p-I_{z z} r+C_{6 \text { trunc }} \quad 0 \quad-I_{x z} r-I_{x y} q+I_{x x} p-C_{4 \text { trunc }} \\
& -I_{y x z} r-I_{x y} p+I_{y y} q-C_{5_{\text {trunc }}} I_{x z} r+I_{x y} q-I_{x x} p+C_{4_{\text {trunc }}} \quad 0
\end{aligned}
$$

where

$$
\begin{aligned}
& C_{1}=X_{\dot{u}} u+X_{\dot{w}} w+X_{\dot{q}} q \\
& C_{2}=Y_{\dot{v}} v+Y_{\dot{p}} p+Y_{\dot{r}} r \\
& C_{3}=X_{\dot{w}} u+Z_{\dot{w}} w+Z_{\dot{q}} q \\
& C_{4}=Y_{\dot{p}} v+K_{\dot{p}} p+K_{\dot{r}} r \\
& C_{5}=X_{\dot{q}} u+Z_{\dot{q}} w+M_{\dot{q}} q \\
& C_{6}=Y_{\dot{r}} v+K_{\dot{r}} p+N_{\dot{r}} r
\end{aligned}
$$

Even with these simplifications, the ship dynamic models contain too many parameters for feasible parameter identification. Since the primary goal of this ship modeling discussion is to provide a suitable structure for a maneuvering model, the roll, pitch, and heave dynamics are ignored in all of the following modeling sections. 


\subsection{Planar Kinematics}
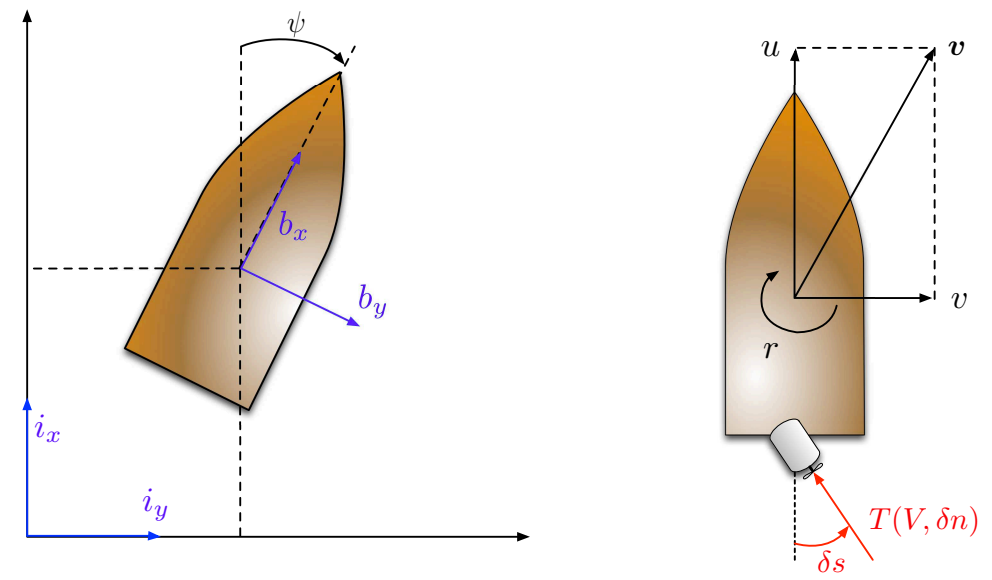

Figure 4.2: Schematic depiction of a USV.

To simplify matters, we will ignore the roll, pitch, and heave dynamics and consider USV motion in the horizontal plane. Figure 4.2 illustrates the state and input variables for a planar USV model with a gimbaled thruster (e.g., an outboard engine). Referring to (4.1) and (4.2), the kinematic model for a USV in planar motion in the absence of currents is

$$
\left(\begin{array}{c}
\dot{x} \\
\dot{y} \\
\dot{\psi}
\end{array}\right)=\left(\begin{array}{ccc}
\cos \psi & -\sin \psi & 0 \\
\sin \psi & \cos \psi & 0 \\
0 & 0 & 1
\end{array}\right)\left(\begin{array}{l}
u \\
v \\
r
\end{array}\right)
$$

With the total velocity $V=\sqrt{u^{2}+v^{2}}$, we define the sideslip angle $\beta$ and the course angle $\chi$ as follows:

$$
\beta=\arcsin \left(\frac{v}{V}\right) \quad \text { and } \quad \chi=\psi+\beta
$$

These definitions are illustrated in Figure 4.3. Note that the course angle and the heading angle are equal 
unless the vehicle is slipping. Recognizing that

$$
\left(\begin{array}{l}
u \\
v
\end{array}\right)=\left(\begin{array}{cc}
\cos \beta & -\sin \beta \\
\sin \beta & \cos \beta
\end{array}\right)\left(\begin{array}{l}
V \\
0
\end{array}\right),
$$

we may write

$$
\left(\begin{array}{c}
\dot{x} \\
\dot{y} \\
\dot{\psi}
\end{array}\right)=\left(\begin{array}{ccc}
\cos \psi & -\sin \psi & 0 \\
\sin \psi & \cos \psi & 0 \\
0 & 0 & 1
\end{array}\right)\left(\begin{array}{ccc}
\cos \beta & -\sin \beta & 0 \\
\sin \beta & \cos \beta & 0 \\
0 & 0 & 1
\end{array}\right)\left(\begin{array}{l}
V \\
0 \\
r
\end{array}\right)
$$

or

$$
\left(\begin{array}{l}
\dot{x} \\
\dot{y} \\
\dot{\chi}
\end{array}\right)=\left(\begin{array}{ccc}
\cos \chi & -\sin \chi & 0 \\
\sin \chi & \cos \chi & 0 \\
0 & 0 & 1
\end{array}\right)\left(\begin{array}{c}
V \\
0 \\
r+\dot{\beta}
\end{array}\right)
$$

where

$$
V \cos \beta \dot{\beta}=\dot{v}-\dot{V} \sin \beta
$$

The speed-and-sideslip representation provides a useful alternative view, despite the ambiguity in the definition of $\beta$ when $V \rightarrow 0$. Note that (4.4) is not really a kinematic model, though, because $\dot{\beta}$ depends on acceleration as well as velocity.

\subsection{Planar Nonlinear 3 DOF Dynamics}

By truncating the 6 DOF nonlinear model to the 3 DOF that define maneuvering motion we inevitably loose fidelity while gaining simplicity. For instance, thrust line effects, which makes smaller boats lean into turns, are neglected. Since the thrust is coming from below the center of gravity and the turning moment is due to a force opposing the direction of the turn, the boat naturally leans into the turn. "Sea-keeping" behavior describing motion due to waves and metacentric stability is also neglected. With these effects ignored the 


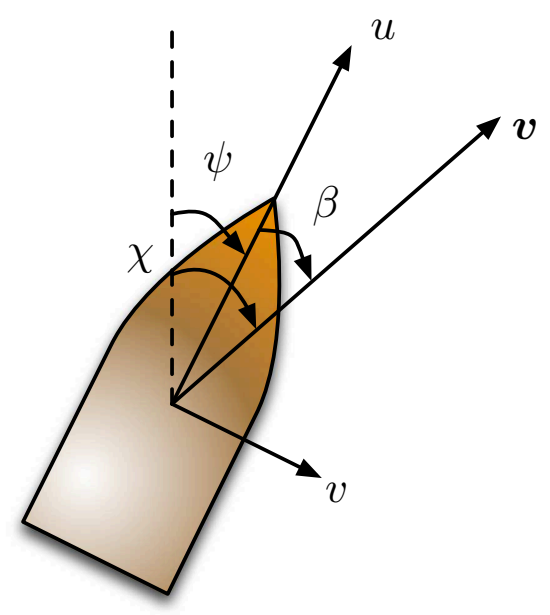

Figure 4.3: Definitions of side slip and course angles.

pitch, roll, and heave equations of motion become

$$
\begin{aligned}
Z & =K=M=0 \\
p & =q=w=0 \\
\boldsymbol{g}(\boldsymbol{\eta}) & =\mathbf{0}
\end{aligned}
$$

with the 3 DOF state vectors

$$
\begin{aligned}
& \boldsymbol{\nu}=\left(\begin{array}{lll}
u & v & r
\end{array}\right)^{T} \\
& \boldsymbol{\eta}=\left(\begin{array}{lll}
x & y & \psi
\end{array}\right)^{T}
\end{aligned}
$$

which are truncations of the 6 DOF state vectors, though the original notation is retained. The mass matrix for this planar configuration is

$$
\boldsymbol{M}=\left(\begin{array}{ccc}
m-X_{\dot{u}} & 0 & 0 \\
0 & m-Y_{\dot{v}} & m x_{G}-N_{\dot{v}} \\
0 & m x_{G}-N_{\dot{v}} & I_{z}-N_{\dot{r}}
\end{array}\right)
$$


The Coriolis and centripetal matrix is

$$
\boldsymbol{C}(\boldsymbol{\nu})=\left(\begin{array}{ccc}
0 & 0 & -m\left(x_{\mathrm{cg}} r+v\right)+Y_{\dot{v}} v+\frac{N_{\dot{v}}+Y_{\dot{r}}}{2} r \\
0 & 0 & \left(m-X_{\dot{u}}\right) u \\
m\left(x_{\mathrm{cg}} r+v\right)-Y_{\dot{v}} v-\frac{Y_{\dot{r}}+N_{\dot{v}}}{2} r & \left(X_{\dot{u}}-m\right) u & 0
\end{array}\right)
$$

The original 6 DOF drag matrix can be further simplified by exploiting the assumption that forward drag decouples from turning and sideslip drag giving

$$
\boldsymbol{D}_{l}=-\left(\begin{array}{ccc}
X_{u} & 0 & 0 \\
0 & Y_{v} & Y_{r} \\
0 & N_{v} & N_{r}
\end{array}\right)
$$

for linear drag and

$$
\boldsymbol{D}_{q}(\nu)=\left(\begin{array}{ccc}
X_{u|u|}|u| & 0 & 0 \\
0 & Y_{v|v|}|v| & Y_{r|r|}|r| \\
0 & N_{v|v|}|v| & N_{r|r|}|r|
\end{array}\right)
$$

for quadratic drag.

For a vessel with a steerable outboard motor, the control forces and moment depend on the propeller speed $\delta n$ and the thrust deflection angle $\delta s$. Details concerning the actuator model are given next, in Section 4.5. 
The three DOF equations of motion are:

$$
\begin{array}{ll}
\left(m-X_{\dot{u}}\right) \dot{u}-m\left(x_{\mathrm{cg}} r^{2}+v r\right)+Y_{\dot{v}} v r+\frac{Y_{\dot{r}}+N_{\dot{v}}}{2} r^{2}+X_{u} u+X_{|u| u \mid}|u| u & =X_{\mathrm{ctrl}}(\delta r, \delta n) \\
\left(m-Y_{\dot{v}}\right) \dot{v}+\left(m x_{\mathrm{cg}}-Y_{\dot{r}}\right) \dot{r}+\left(m-X_{\dot{u}}\right) u r+Y_{v} v+Y_{r} r+Y_{|v| v|v| v+Y_{|r| r \mid}|r| r} & =Y_{\mathrm{ctrl}}(\delta r, \delta n) \\
\left(m x_{\mathrm{cg}}-N_{\dot{v}}\right) \dot{v}+\left(I_{z z}-N_{\dot{r}}\right) \dot{r}+m x_{\mathrm{cg}} u r-Y_{\dot{v}} u v-\frac{Y_{\dot{r}}+N_{\dot{v}}}{2} u r+X_{\dot{u}} u v & \\
+N_{v} v+N_{r} r+N_{|v| v}|v| v+N_{|r| r \mid}|r| r & =N_{\mathrm{ctrl}}(\delta r, \delta n)
\end{array}
$$

Though the nonlinear equations (4.5), (4.6), and (4.7) are based on physical principles, the hydrodynamic effects are modeled rather simplistically, based on reduced complexity models developed over the years for displacement vessels. The nonlinear model of Abkowitz [Abkowitz, 1964] includes a third-order truncated Taylor series expansion of the hydrodynamic forces and moment. Reduced complexity variants, in which only the essential, empirically observed hydrodynamic effects are retained, are described in [Norrbin, 1970] and [Blanke, 1981]. Because of the importance of wave-induced roll coupling in ocean vessel dynamics, a number of models have been developed to capture coupling with this additional DOF [Amerongen and Cappelle, 1981, Son and Nomoto, 1981, Christensen and Blanke, 1986]. Considering operations in the relatively confined environment of a river, we neglect any coupling between horizontal plane motions (surge, sway, and yaw) and rolling motion.

Models that accurately exhibit nonlinear hydrodynamic effects is an active research field. Many researchers take a numeric approach similar to CFD approaches used to model aircraft forces and moments. Papers include [Katayama et al., 2001, Katayama et al., 2002] for modeling transverse proposing effects of a planing vessel, [S.Sutulo and Soares, 2005], which characterizes a 6 DOF model for catamaran motion, [Ayaz et al., 2006, Armaoglu et al., 2006] for 6 DOF motion that considers frequency dependent phenomena with memory effects in random waves in astern seas for semi-displacement vessels, and [Javanmardi et al., 2008], which models distinctive behavior of a trimaran vessel. Models of these complex hydrody- 
namic effects can also be determined through experimentation in tow tanks. Papers include [Ikeda et al., 2000], which displays results for very high speed planing vessel tests based on the planar motion mechanism (PMM) test and [T.Katayama et al., 2000], which uses the PMM test for unstable periodic motions of high speed vessels.

\subsection{Actuation}

The propeller thrust and angle define the control forces and moment appearing in equations (4.5), (4.6), and (4.7). A typical outboard motor includes a small vertical surface (a rudder) which generates a hydrodynamic side force that provides some stability and control. We assume that propeller thrust generates the entire turning moment, though one could easily incorporate the effect of rudder force if needed.

Thrust $T$ is a function of the vessel speed $V$ and the propeller speed $\delta n$ as described in Fossen [Fossen, 1991]:

$$
T=\rho D^{4} K_{T}\left(J_{0}\right)|\delta n| \delta n
$$

where $\rho$ is water density, $D$ is the diameter of the propeller, and $K_{T}$ is a thrust coefficient which depends on the advance ratio

$$
J_{0}=\frac{V(1-w)}{\delta n D}
$$

In the definition of $J_{0}$, the dimensionless "wake fraction number" $w<1$ accounts for the relative reduction in flow velocity into the propeller due to the vessel's wake. In general, the thrust coefficient is a nonlinear function of the advance ratio, but Fossen [Fossen, 1991] suggests $K_{T}$ is well-modeled as a linear function

$$
K_{T}=\alpha_{1}+\alpha_{2} J_{0}
$$

particularly when $J_{0}>0$, that is, when the direction of the vessel motion is commensurate with propeller rotation. However, the actual values of $\alpha_{1}$ and $\alpha_{2}$ might change with the sign of $J_{0}$; propellers and vessel hulls are typically designed to be most efficient in forward motion and will have different parameters describe their thrust for each direction of rotation. 
Using (4.8), one finds that

$$
T=c V \delta n+d|\delta n| \delta n
$$

where

$$
\begin{aligned}
& c=(1-w) \rho D^{3} \alpha_{2} \\
& d=\rho D^{4} \alpha_{1}
\end{aligned}
$$

For model identification purposes, it will be sufficient to find the two parameters $c$ and $d$, rather than the five parameters $w, \rho, D, \alpha_{1}$, and $\alpha_{2}$.

The control forces and moment in equations (4.5), (4.6), and (4.7) are

$$
\begin{aligned}
X_{\mathrm{ctrl}} & =(c V \delta n+d|\delta n| \delta n) \cos \delta r \\
Y_{\mathrm{ctrl}} & =(c V \delta n+d|\delta n| \delta n) \sin \delta r \\
N_{\mathrm{ctrl}} & =x_{\delta r}(c V \delta n+d|\delta n| \delta n) \sin \delta r
\end{aligned}
$$

where $x_{\delta r}$ is the longitudinal moment arm from the center of rotation to the pivot point of the thruster.

In the following sections, we present several simplified variants of the dynamic equations (4.5-4.7) with actuation model (4.11-4.13). The objective is to obtain a model that is rich enough to enable effective motion planning and control, simple enough to allow quick identification, and general enough to describe a variety of vehicles operating over a large range of speeds.

\subsection{Simplified Vessel Dynamics}

Planing dynamics exist in powerful, purpose built small to medium boats including the Ribcraft USV. At slow speeds these boats act similarly to large boats that drag themselves through the water, completely relying upon buoyancy to stay afloat. At higher speeds the small to medium boats are pushed up out of the water with the hull of the boat skidding across the water. To add to the complexity of these vessels' dynamics, their steady state pitch angle changes based upon speed, changing the planform exposed to the 
water and consequently changing the added mass terms. With these added complexities a speed scheduled set of parameters for simplified steering and speed models is sought as an alternative to the nonlinear planar 3 DOF model.

\subsubsection{Speed Dynamics}

Simplified forward speed models retain drag terms but exclude terms that couple forward velocity with side velocity and turn rate.

\subsubsection{Quadratic Drag Speed Model}

For straight line motion, equation (4.5) simplifies to

$$
m \dot{u}=X_{\dot{u}} \dot{u}+X_{u} u+X_{|u| u \mid}|u| u+(c u \delta n+d|\delta n| \delta n)
$$

If the turn rate $r$ and the side velocity $v$ are small, the speed model (4.14) continues to be a valid representation of (4.5). Note that (4.14) contains the thrust model shown in Section 4.5. This thruster model will also be vital in the steering modeling section.

\subsubsection{Linear Drag Speed Model}

Linearizing equation (4.5) about the steady motion corresponding to the state and input values

$$
u=u_{0}, \quad v=0, \quad r=0, \quad \delta r=0, \quad \text { and } \quad T=T_{0}
$$

one obtains the perturbation dynamics

$$
\left(m-X_{\dot{u}}\right) \Delta \dot{u}=\left(X_{u}+2 X_{u|u|} u_{0}\right) \Delta u+\Delta T
$$

where

$$
u=u_{0}+\Delta u \quad \text { and } \quad T=T_{0}+\Delta T
$$


Note that small perturbations in $v$ and $r$ do not affect $u$; again, the speed dynamics decouple from the steering dynamics.

Equation (4.16) provides a first order linear model relating propeller speed to vehicle speed, in the neighborhood of the nominal condition (4.15):

$$
\Delta \dot{u}=a_{u} \Delta u+b_{u} \Delta T
$$

Writing equation (4.17) in terms of the original (non-perturbation) variables gives

$$
\dot{u}=a_{u} u+b_{u} T-\underbrace{\left(a_{u} u_{0}+b_{u} T_{0}\right)}_{b_{u_{\text {bias }}}}
$$

Model identification requires finding the three parameters $a_{u}, b_{u}$, and $b_{u_{\text {bias }}}$. While this (speed-parameterized) first order, linear time-invariant system is a standard model, other variations have been proposed; see Breivik et. al. [Breivik et al., 2008], for example. For cases where the vessel drag force is a complicated function of forward velocity, this model, used with a speed scheduled set of parameters, can adequately estimate forward speed dynamics.

\subsubsection{Steering Dynamics}

Having modeled the vessel's motion along the longitudinal axis, it remains to determine the steering model, describing sway and yaw motions. As in the simplified speed dynamics section, the steering dynamics are decoupled from forward velocity. Like the simplified speed models only drag and mass matrix terms survive linearization with the coupling terms terms from the Coriolis and centripetal matrix excluded. 


\subsubsection{Linear Steering Dynamics}

Simply linearizing (4.6) and (4.7) about the steady motion (4.15) gives

$$
\left(\begin{array}{ccc}
m-Y_{\dot{v}} & 0 & m x_{\mathrm{cg}}-Y_{\dot{r}} \\
0 & 1 & 0 \\
m x_{\mathrm{cg}}-N_{\dot{v}} & 0 & I_{z}-N_{\dot{r}}
\end{array}\right)\left(\begin{array}{c}
\dot{v} \\
\dot{\psi} \\
\dot{r}
\end{array}\right)+\left(\begin{array}{ccc}
-Y_{v} & 0 & m u_{0}-Y_{r} \\
0 & 0 & 1 \\
-N_{v} & 0 & m x_{\mathrm{cg}} u_{0}-N_{r}
\end{array}\right)\left(\begin{array}{l}
v \\
r
\end{array}\right)=\left(\begin{array}{c}
1 \\
0 \\
x_{\delta r}
\end{array}\right) T_{0} \delta r
$$

Writing (4.19) in the form

$$
\left(\begin{array}{c}
\dot{v} \\
\dot{\psi} \\
\dot{r}
\end{array}\right)=\boldsymbol{A}\left(\begin{array}{c}
v \\
\psi \\
r
\end{array}\right)+\boldsymbol{B} T_{0} \delta r
$$

one finds that there are six elements of $\boldsymbol{A}$ and $\boldsymbol{B}$ to be identified. Anticipating that there may some bias in the steering dynamics, such that a turning moment is induced even when the the rudder is centered, equation (4.20) can be augmented as follows

$$
\left(\begin{array}{c}
\dot{v} \\
\dot{\psi} \\
\dot{r}
\end{array}\right)=\boldsymbol{A}\left(\begin{array}{c}
v \\
\psi \\
r
\end{array}\right)+\boldsymbol{B} T_{0} \delta r+\boldsymbol{B}_{\mathrm{bias}}
$$

In this case, two additional parameters must be identified to determine $\boldsymbol{B}_{\mathrm{bias}}$.

\subsubsection{Nomoto's Steering Models}

Considering large displacement vessels, Nomoto and colleagues [Nomoto et al., 1957] suggested that turn rate can be described as a simple first order equation relating the rudder angle $\delta r$ to the turn rate $r$ at a given forward speed $u_{0}$ :

$$
\tau \dot{r}+r=K_{r} \delta r
$$

where

$$
K_{r}=\frac{T_{0} x_{\delta r}}{I_{z z}-N_{\dot{r}}}
$$


Adding an integrator to incorporate heading information gives

$$
\left(\begin{array}{c}
\dot{\psi} \\
\dot{r}
\end{array}\right)=\left(\begin{array}{cc}
0 & 1 \\
0 & -\frac{1}{\tau_{r}}
\end{array}\right)\left(\begin{array}{l}
\psi \\
r
\end{array}\right)+\left(\begin{array}{c}
0 \\
\frac{x_{\delta r}}{\tau_{r}\left(I_{z z}-N_{\dot{r}}\right)}
\end{array}\right) T_{0} \delta r+\left(\begin{array}{c}
0 \\
b_{\mathrm{bias}}
\end{array}\right)
$$

Vessels that steer with a vectored thruster (and possibly a rudder) generally create a nonzero sideslip angle $\beta$ while turning. Nomoto's model assumes, however, that the vessel is constrained to move in the direction in which it is pointed; it cannot slip while turning. To address this deficiency in the Nomoto model (4.22) without resorting to the full linearized steering model (4.21), one can incorporate a first order lag model for sideslip [Yu et al., 2008b]:

$$
\tau_{\beta} \dot{\beta}+\beta=-K_{\beta} r
$$

Combining equations (4.22) and (4.23) gives the system

$$
\left(\begin{array}{c}
\dot{\beta} \\
\dot{\psi} \\
\dot{r}
\end{array}\right)=\left(\begin{array}{ccc}
-\frac{1}{\tau_{\beta}} & 0 & -\frac{K_{\beta}}{\tau_{\beta}} \\
0 & 0 & 1 \\
0 & 0 & -\frac{1}{\tau_{r}}
\end{array}\right)\left(\begin{array}{l}
\beta \\
\psi \\
r
\end{array}\right)+\left(\begin{array}{c}
0 \\
0 \\
\frac{x_{\delta r}}{\tau_{r}\left(I_{z z}-N_{\dot{r}}\right)}
\end{array}\right) T_{0} \delta r+\left(\begin{array}{c}
b_{1_{\text {bias }}} \\
0 \\
b_{2_{\text {bias }}}
\end{array}\right)
$$

Note that, like (4.16) these linear steering models can capture complex dynamics not described by (4.5), (4.6), and (4.7), by using a speed scheduled set of steering parameters. An additional example of the need for parameter scheduling of simplified models will be shown in the subsequent section describing sternward dynamics of the Ribcraft USV.

\subsubsection{Considering Sternward Steering Behavior}

The Ribcraft USV is intended to operate in potentially unknown riverine environments, which includes confined areas that do not contain a navigable passage to the desired destination, requiring the vehicle to first back up and then turn around. A dynamic model for reverse motion is needed in order to safely execute sternward maneuvers.

The Ribcraft USV exhibits a bifurcation in equilibria when driving in reverse. With the rudder centered 
and the throttle fixed at a setting that results in sternward motion, the vessel may enter into either a left or right turn. Once turning motion is established, significant rudder input is required to turn in the opposite direction.

Investigation of this phenomenon is necessary because simple linear controllers that do not account for these unique dynamics cannot track desired headings. If the bifurcation causes the boat to be uncontrollable then reverse motion should be avoided altogether. However, the dynamics of the boat are slow enough to be within the bandwidth of human control, leading to the conclusion that a more advanced controller, coupled with a dynamic model or set of dynamic models, might give reasonable tracking.

As a mechanical analog to the surface vessel moving in reverse, first consider a planar pendulum that is spinning about its vertical axis. This well-known dynamical system, a simple model of Watt's flyball regulator [Beltrami, 1998], exhibits a pitchfork bifurcation when the spin rate exceeds a critical value. If the pendulum pivot is offset from the spin axis, the pitchfork bifurcation degenerates into a saddle-node bifurcation. In our analogy, the spin rate of the pendulum is similar to the backward speed of the surface vessel while the pendulum offset is analogous to the steering angle.

Using insight provided by the whirling planar pendulum dynamics, a set of linear steering models, which contain more tuning parameters than the planar pendulum formulation, estimate the sternward bistable dynamics. This set of linear steering models includes an unstable steering model at the zero turn rate equilibrium sandwiched between two stable left and right turn biased steering models. A switching envelope around the unstable zero turn rate equilibrium connects the three models in state space.

A set of model structures estimating vessel steering and speed dynamics has been developed, but these models can accurately estimate the Ribcraft USV motion only after their parameters have been determined by using an experimental system identification method. The method used to identify motion parameters for the Ribcraft USV is described in the following chapter. 


\section{CHAPTER}

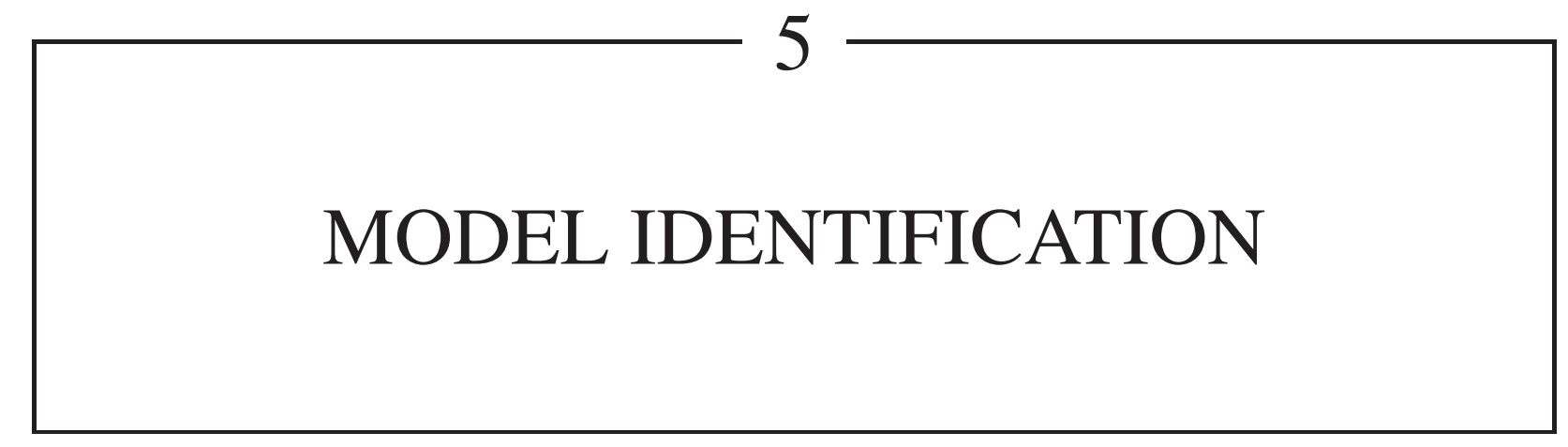

The USV model parameter identification method is broken down into two steps. The first step is to determine the steady-state relationship between inputs and outputs from open-loop maneuvers. With the "DC gains" determined, the second step is to identify any additional parameters that govern transient behavior. Inspired by previous system identification efforts for autonomous underwater vehicles [Petrich, 2009, Petrich and Stilwell, 2011], we implement simple controllers for motion variables of interest (e.g., speed and heading) and obtain closed-loop state histories corresponding to specified, desired output histories (e.g., a square wave in heading angle). Using closed-loop system responses instead of open-loop responses allows more control over the vehicle's motion in the confined operating environments where field tests are performed.

The Ribcraft USV's actuators have nonlinear characteristics such as rate limiting, saturation, time delay, and deadband. These nonlinear effects can be determined experimentally by simply comparing actual and commanded inputs. Actuator nonlinearities are not explicitly used in the dynamic models, but are instead 
applied to vessel simulations used in preliminary gain tuning and feasible trajectory generation. These actuator limitations have an unavoidable effect on closed-loop behavior, requiring time consuming gain tuning.

When estimating model parameters, the question of identifiability naturally arises. For linear dynamic models, as discussed in Petrich [Petrich, 2009], it is sufficient to verify that the system's Markov parameters depend appropriately on the parameters to be identified [Grewal and Glover, 1976]. For nonlinear dynamic models, one may consider local identifiability by linearizing around a nominal state. For the USV, identifiability analysis is straightforward and is included in Appendix A.

\subsection{Identifying Steady-State Input/Output Relationships}

To determine the steady-state relationship between inputs and states for a stable system, one applies a constant input and measures the steady-state output. For the vectored thrust USV considered here, the two constant inputs are the thrust angle $\delta s$ and the propeller speed $\delta n$. Performing these steady-state experiments over a range of thrust angles and propeller speeds defines a two-parameter family of steady-state operating conditions.

We begin by considering linear, time-invariant systems with bias

$$
\dot{\boldsymbol{x}}=\boldsymbol{A} \boldsymbol{x}+\boldsymbol{B u}+\boldsymbol{B}_{\mathrm{bias}}
$$

where $\boldsymbol{x}(t) \in \mathbb{R}^{n}, \boldsymbol{u}(t) \in \mathbb{R}^{m}$, and $\boldsymbol{A}, \boldsymbol{B}$, and $\boldsymbol{B}_{\text {bias }}$ have commensurate dimensions. The identification task is to determine the $n(n+m+1)$ parameters appearing in $\boldsymbol{A}, \boldsymbol{B}$ and $\boldsymbol{B}_{\mathrm{bias}}$. Note that if the only biases are in the inputs and are known then the relationship $\boldsymbol{B}_{\text {bias }}=\boldsymbol{B} u_{\text {bias }}$, where $u_{\text {bias }}$ is an input offset, excludes bias from being directly identified. However, the additional parametric freedom provided by $\boldsymbol{B}_{\text {bias }}$ results in better matches of the vehicle's steady-state performance. A primary requirement is that the resulting parameter values should recover observed steady-state behavior.

Assuming that the matrix $\boldsymbol{A}$ in (5.1) is Hurwitz, a constant input $\boldsymbol{u}_{\mathrm{ss}}$ will eventually result in a steady 
state

$$
\boldsymbol{x}_{\mathrm{ss}}=\boldsymbol{\Phi} \boldsymbol{u}_{\mathrm{ss}}+\boldsymbol{\Phi}_{\mathrm{bias}}
$$

For a sufficiently rich set of inputs $\boldsymbol{u}_{\mathrm{ss}}$, one may determine the $n(m+1)$ parameters defining the matrix $\boldsymbol{\Phi}=\boldsymbol{A}^{-1} \boldsymbol{B}$ and the vector $\boldsymbol{\Phi}_{\text {bias }}=\boldsymbol{A}^{-1} \boldsymbol{B}_{\text {bias }}$ from experimental data. These steady-state parameters may be used to constrain the remaining identification process. Noting that

$$
\begin{aligned}
\mathbf{0} & =\boldsymbol{A} \boldsymbol{x}_{\mathrm{sS}}+\boldsymbol{B} \boldsymbol{u}_{\mathrm{ss}}+\boldsymbol{B}_{\mathrm{bias}} \\
& =(\boldsymbol{A} \boldsymbol{\Phi}+\boldsymbol{B}) \boldsymbol{u}_{\mathrm{ss}}+\left(\boldsymbol{A} \boldsymbol{\Phi}_{\mathrm{bias}}+\boldsymbol{B}_{\mathrm{bias}}\right)
\end{aligned}
$$

for $m$ linearly independent inputs, the following $n(m+1)$ linear equations constrain the choice of the $n(n+m+1)$ parameters appearing in $\boldsymbol{A}, \boldsymbol{B}$ and $\boldsymbol{B}_{\text {bias }}$ :

$$
\begin{aligned}
& \mathbf{0}=\boldsymbol{A} \Phi+\boldsymbol{B} \\
& \mathbf{0}=\boldsymbol{A} \boldsymbol{\Phi}_{\text {bias }}+\boldsymbol{B}_{\text {bias }}
\end{aligned}
$$

Incorporating these constraints leaves $n^{2}$ free parameters to be determined from dynamic (transient) system response data.

Note that unconstrained parameter identification will always minimize the equation error to a lower value than constrained identification since the unconstrained approach finds parameters that give a match weighing the steady state/transient trade off based upon the makeup of transient and steady behavior contained in the particular data set used. Constraining parameters of the estimated models forces steady state behavior to match exactly, regardless of the content of any particular data set. In fact, after steady state data has been collected and analyzed, experiments can be designed that have little to no steady state motion, yet the constrained approach will still yield correct steady state behavior. 


\subsection{Identifying Dynamic Parameters}

Having found the steady state parameters appearing in $\boldsymbol{\Phi}$ and $\boldsymbol{\Phi}_{\text {bias }}$, one seeks the remaining "dynamic parameters" appearing in the model (5.1). We begin with the thruster dynamic model, which requires special consideration, and then describe identification of the steering and speed dynamics.

Since the identification scheme does not directly consider noise in inputs, states, and state derivatives but experimental measurements are noisy and state derivatives are not directly measured, experimental data must be filtered to smooth the data and generate estimates of time derivatives. To simultaneously smooth and differentiate measurements, we use the Savitzky-Golay filter [Savitzky and Golay, 1964]. This filter uses least squares regression to fit a polynomial to signal data within a moving window; the time derivative is estimated using the derivative of the polynomial.

\subsubsection{Identifying Thruster Parameters}

In this section, we consider identification of the thruster model (4.10). Consider the speed dynamics (4.5) for the case of straight line motion :

$$
\dot{u}=\frac{X_{u}}{\left(m-X_{\dot{u}}\right)} u+\frac{X_{|u| u}}{\left(m-X_{\dot{u}}\right)}|u| u+\frac{T}{\left(m-X_{\dot{u}}\right)}
$$

The parameters $X_{\dot{u}}, X_{u}$, and $X_{|u| u}$ may be considered coefficients in a polynomial expansion of the hydrodynamic surge force around a nominal condition. Because the USV is a planing vessel, the waterline will vary with the nominal state. These "hydrodynamic derivatives" are therefore parameterized by the nominal forward speed $u_{0}$. The thrust term $\tilde{T}$ is

$$
\begin{aligned}
\tilde{T} & =\frac{T}{m-X_{\dot{u}}} \\
& =\underbrace{\frac{c}{\left(m-X_{\dot{u}}\right)}}_{\tilde{c}} u \delta n+\underbrace{\frac{d}{\left(m-X_{\dot{u}}\right)}}_{\tilde{d}}|\delta n| \delta n
\end{aligned}
$$

To identify the parameters $\tilde{c}$ and $\tilde{d}$ in (5.7), one may isolate dynamics due to thrust by examining the initial 
response to step inputs in throttle, starting from straight line motion with a steady speed $u_{0}$ corresponding to a fixed thrust $\tilde{T}_{0}$ :

$$
0=\frac{X_{u}}{\left(m-X_{\dot{u}}\right)} u_{0}+\frac{X_{|u| u}}{\left(m-X_{\dot{u}}\right)}\left|u_{0}\right| u_{0}+\tilde{T}_{0}
$$

Substituting perturbation values

$$
u=u_{0}+\Delta u \quad \text { and } \quad \tilde{T}=\tilde{T}_{0}+\Delta \tilde{T}
$$

into the dynamic equation (5.6) gives

$$
\Delta \dot{u}=\frac{X_{u}}{\left(m-X_{\dot{u}}\right)}\left(u_{0}+\Delta u\right)+\frac{X_{|u| u}}{\left(m-X_{\dot{u}}\right)}\left|\left(u_{0}+\Delta u\right)\right|\left(u_{0}+\Delta u\right)+\left(\tilde{T}_{0}+\Delta \tilde{T}\right)
$$

Subtracting the steady state condition (5.8), assuming that $u_{0}>0$ is much larger than $|\Delta u|$, and ignoring higher order perturbation terms leaves

$$
\Delta \dot{u}=\tilde{a} \Delta u+\Delta \tilde{T} \quad \text { with } \quad \tilde{a}=\frac{1}{\left(m-X_{\dot{u}}\right)}\left(X_{u}+2 X_{|u| u} u_{0}\right)
$$

It is straight forward to verify that, in the initial limit,

$$
\dot{u}(0)=\Delta \tilde{T}
$$

Thus, with high rate data for the vessel speed $u$ and the propeller speed $\delta n$, and using a Savitzky-Golay filter to determine the acceleration $\dot{u}$, one obtains a collection of initial accelerations corresponding to step inputs in throttle. Recalling the thrust relation (5.7) and applying the pseudoinverse, one obtains a least square error solution to the over-determined linear algebraic system for $\tilde{c}$ and $\tilde{d}$. Experimental identification results for the Ribcraft USV verify that $\tilde{c}$ and $\tilde{d}$ do not vary with the nominal speed, even in planing mode, making $\tilde{T}$ differ from $T$ by a constant scale factor. This scale factor can be absorbed into the input parameters of the steering and speed models in Section 4.6, allowing $\tilde{T}$ to replace $T$ for system identification. 


\subsubsection{Identifying Linear State Transient Dynamics}

Referring to (5.1), suppose we have obtained sampled data for a state history $\boldsymbol{x}(t)$ and, using the SavitzkyGolay algorithm, the corresponding state rate history $\dot{\boldsymbol{x}}(t)$ in response to some suitably rich input history $\boldsymbol{u}(t)$. These data define a system of algebraic equations for the parameters appearing in $\boldsymbol{A}$ and $\boldsymbol{B}$. At each of the $N$ sample times $t_{i}$, for $i \in\{1, \ldots, N\}$, one has

$$
\dot{\boldsymbol{x}}\left(t_{i}\right)=\boldsymbol{A} \boldsymbol{x}\left(t_{i}\right)+\boldsymbol{B} \boldsymbol{u}\left(t_{i}\right)+\boldsymbol{B}_{\mathrm{bias}}
$$

Incorporating the steady-state constraints (5.4) and (5.5) by substituting

$$
\boldsymbol{B}=-\boldsymbol{A} \Phi \quad \text { and } \quad \boldsymbol{B}_{\text {bias }}=-\boldsymbol{A} \boldsymbol{\Phi}_{\text {bias }}
$$

gives $n N$ equations

$$
\dot{\boldsymbol{x}}\left(t_{i}\right)=\boldsymbol{A}\left(\boldsymbol{x}\left(t_{i}\right)-\boldsymbol{\Phi} \boldsymbol{u}\left(t_{i}\right)-\boldsymbol{\Phi}_{\text {bias }}\right)
$$

for the $n^{2}$ parameters of $\boldsymbol{A}$. A least square estimate for these parameters can be obtained using the pseudoinverse [Stengel, 1986] for $N>n$. Of course, the absolute accuracy of the estimate depends on the system structure and the data quality. An input history that excites all of the dynamic modes will yield much better parameter estimates, as can be verified using independent validation data.

\subsection{Model Identification Results}

Field trials for the Ribcraft USV were performed in order to compare the various simple models first described in Chapter 4. Section 5.3.1 describes an experimentally obtained steady-state map which relates the fixed values of throttle, propeller speed, and thrust angle to steady turn rate, forward velocity, and side velocity. Actuator dynamics, found by applying step thrust angle or throttle commands while recording the actual thrust angle or propeller speed, are discussed in Section 5.3.2. Section 5.3.3 shows parameter fits for the thruster model. Section 5.3.4 presents simplified vehicle dynamic model parameters deduced from closed-loop field trials involving commanding square waves in heading and velocity while recording time 
histories of thrust angle, propeller speed, orientation, and velocity. Finally, the unique sternward dynamics of the Ribcraft USV are considered in Section 5.3.5 by extending analysis of steady state behavior, thruster modeling, and transient dynamics for bifurcated turn rate behavior.

\subsubsection{Steady-State Input/Output Relationships}

To obtain steady-state motion data, the following sequence of open-loop experiments was performed.

1. Set $\delta \tilde{s}=0^{\circ}$ with the USV heading in a safe direction at idle speed.

2. Increment throttle by a fixed amount (e.g., 5\%). Allow the speed to reach steady-state.

a) Increment $\delta \tilde{s}$ by a fixed amount (e.g., $\left.7.5^{\circ}\right)$.

b) Set $\delta s=\delta \tilde{s}$ and wait for the USV to complete two circular paths.

c) If $\delta s$ is not saturated, return to Step 2(a). Else, continue

d) If $\delta s$ is at maximum, set $\delta s=0$ and return to Step 2(a) incrementing by negative thrust angle values. Else, continue

3. If the throttle is not saturated, return to Step 2. Else, end the test sequence.

Steady-state motion data for the Ribcraft USV are given in Figure 5.1; tabulated steady state gain values are given in Table 5.1. The vessel's throttle has a dead band from -20\% to roughly $35 \%$. For identification purposes throttle settings ranged from $35 \%$ to $65 \%$, providing data on the transition from the dead band region into the semi-displacement and planing region. Thrust angle settings ranged from $-30^{\circ}$ to $30^{\circ}$ thrust angle. The steady state linear fits are described by

$$
\begin{aligned}
& r_{\mathrm{ss}}=K_{r} \delta s_{\mathrm{ss}}+K_{r_{\mathrm{bias}}} \\
& \beta_{\mathrm{ss}}=K_{\beta} r_{\mathrm{ss}}+K_{\beta_{\mathrm{bias}}} \\
& v_{\mathrm{ss}}=K_{v} \delta s_{\mathrm{ss}}+K_{v_{\mathrm{bias}}}
\end{aligned}
$$


Table 5.1: DC gains of $\beta$, $v$, and $r$ for the Ribcraft USV.

\begin{tabular}{c|cccccc} 
Forward Speed, $(\mathrm{m} / \mathrm{s})$ & $K_{\beta}$ & $K_{\beta_{\text {bias }}}$ & $K_{v}$ & $K_{v_{\text {bias }}}$ & $K_{r}$ & $K_{r_{\text {bias }}}$ \\
\hline 1.1 & -1.44 & 0.44 & 0.0060 & -0.009 & -0.28 & 0.87 \\
1.5 & -1.36 & 0.62 & 0.0056 & -0.004 & -0.28 & 0.84 \\
2.6 & -0.26 & 1.20 & 0.0060 & 0.031 & -0.60 & 1.97 \\
3.0 & -0.13 & 0.71 & 0.0040 & 0.021 & -0.68 & 2.11 \\
3.2 & 0.00 & 1.08 & -0.0000 & 0.056 & -0.76 & 2.02 \\
4.0 & 0.08 & 1.17 & -0.0036 & 0.080 & -0.76 & 2.03 \\
7.6 & -0.10 & 1.22 & 0.0096 & 0.135 & -0.76 & 2.28 \\
9.2 & -0.12 & 1.11 & 0.0120 & 0.122 & -0.76 & 2.21
\end{tabular}

Values of $r, \beta$, and $v$ are predominantly linear. Note that the units are in deg/s for turn rate, deg for thrust angle and sideslip angle, and m/s for sideslip velocity. High sideslip angles are observed at low speed. Turn rate, $r$, increases with throttle, but sideslip angle, $\beta$, and sideslip velocity, $v$, decrease to negligible values in the semi-displacement region (throttle values of $45 \%, 50 \%, 55 \%$ ).

Figure 5.2 shows steady forward speeds vs. propeller speed for $\delta s=0$ with curve fits for the reverse, displacement, semi-displacement, and planing modes. The good quality of the fits validates consideration of these four distinct speed regions. Note that Froude numbers of 0.45 and 1 (corresponding to speeds of $3.1(\mathrm{~m} / \mathrm{s})$ and $7(\mathrm{~m} / \mathrm{s}))$ define the boundaries between the forward velocity modes.

\subsubsection{Actuator Nonlinearities}

The propulsion and steering assemblies impose physical constraints on propeller speed and thrust angle adjustments. The Ribcraft USV has a an outboard motor that is limited to rotate roughly $\pm 30^{\circ}$ from $\delta s=0^{\circ}$ (perpendicular to the transom of the boat). The outboard's steering angle rate saturates at roughly $\pm 15 \mathrm{deg} / \mathrm{s}$ due to limitations of the hydraulic motor that steers the thrust angle. The thrust angle is tightly controlled by a PID controller when reference commands satisfy these magnitude and rate limits. The throttle commands the manifold pressure in the engine with a delay of roughly 0.5 seconds. Changing gear (forward, neutral, or reverse) gives an additional 1.5 second delay. Furthermore, the steady state relationship between throttle 


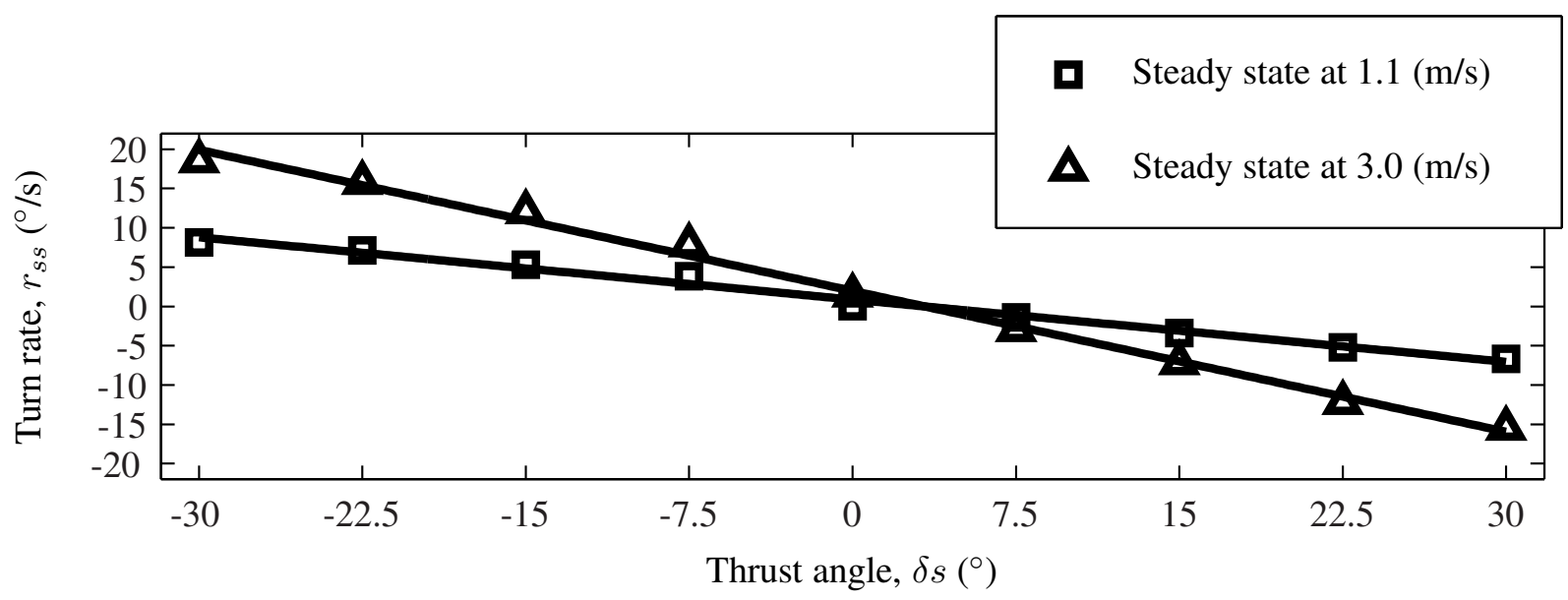

(a) Steady state turn rate vs. thrust angle

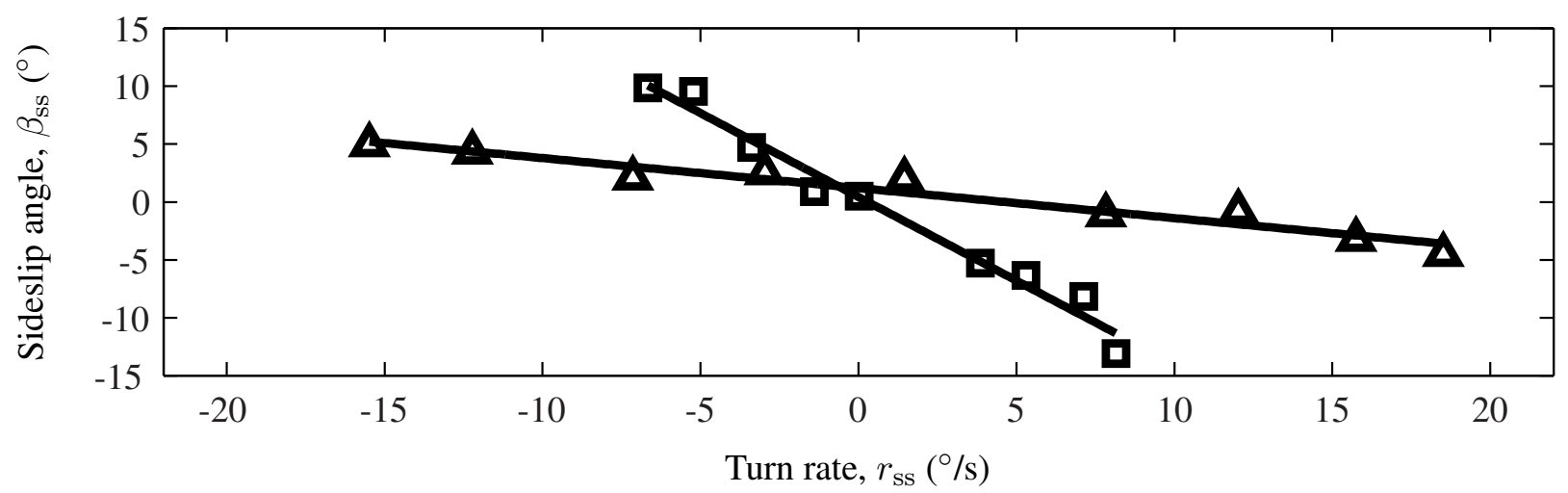

(b) Steady state sideslip angle vs. turn rate

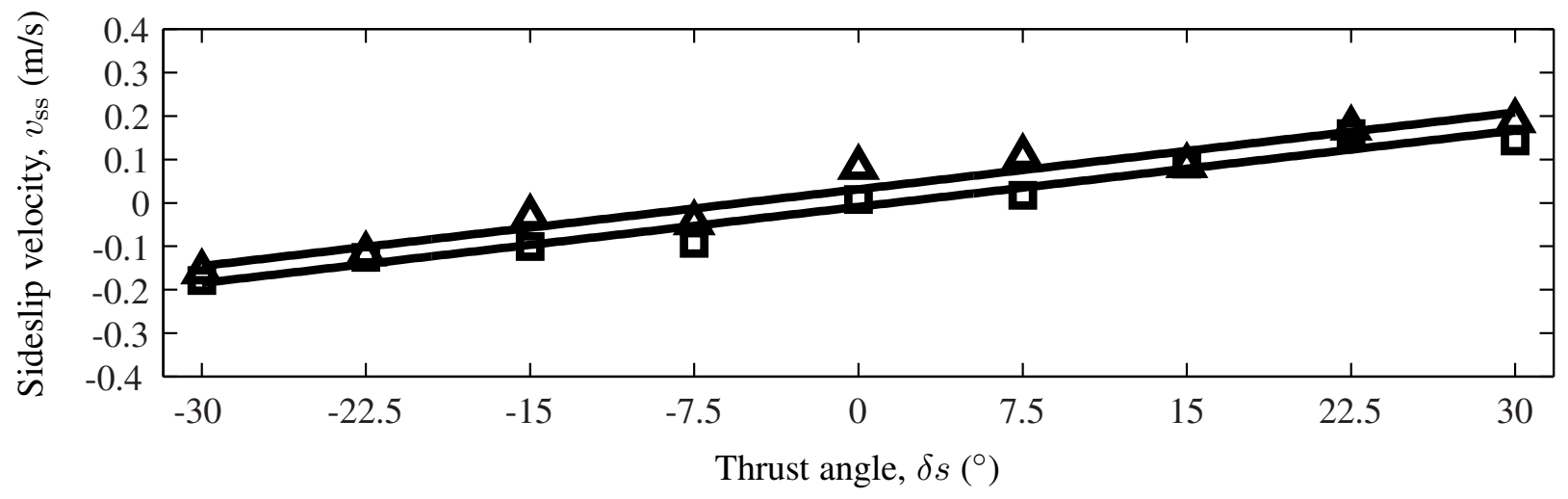

(c) Steady state sideslip velocity vs. thrust angle

Figure 5.1: Steady state relationships for the Ribcraft USV 


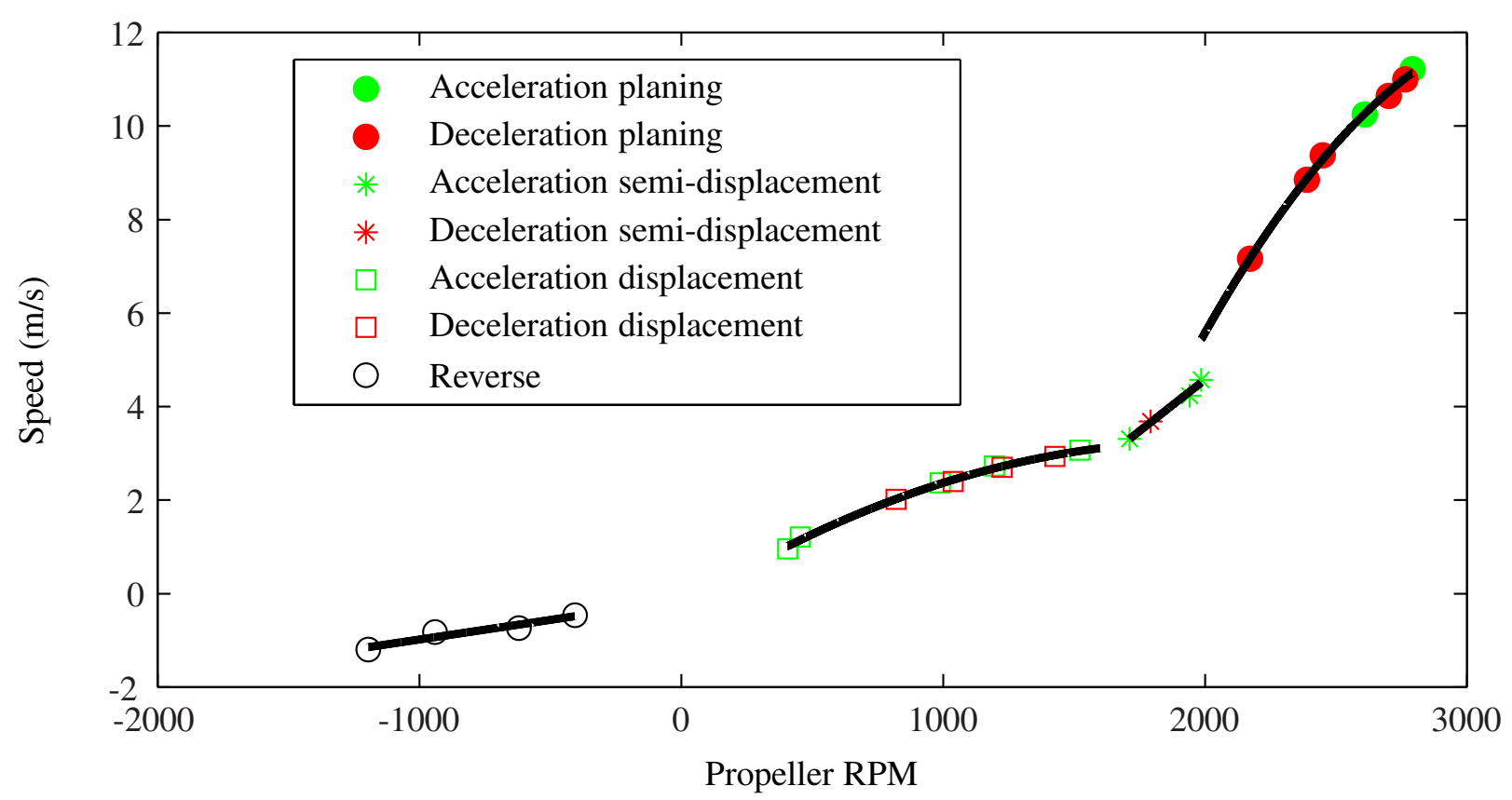

Figure 5.2: Steady state speed fits

position and manifold pressure is nonlinear. Because the manifold pressure and engine dynamics are not measured or estimated, we characterize the steady-state relationship between throttle setting and propeller speed. Throttle is measured in percent; there is a deadband from $-20 \%$ to $35 \%$. When the throttle is at $0 \%$ the engine disengages from the propeller providing no thrust. The engine has hysteresis in the displacement speed region, as can be observed in Figure 5.3. One might expect hysteresis in the throttle/forward speed relationship when passing between the semi-displacement and planing modes, but none is evident. Hysteresis is only apparent in the throttle/propeller speed relationship; see Figures 5.2 and 5.3. The data fits for throttle in reverse, displacement/semi-displacement, and planing modes

$$
\begin{aligned}
& \delta T=0.0184 \delta n-12.88 \\
& \delta T=1.02 \times 10^{-5} \delta n|\delta n|-0.0054 \delta n+37.08 \\
& \delta T=0.0328 \delta n-11.41
\end{aligned}
$$


shown in Figure 5.3 define the steady-state relationship between throttle and propeller speed outside the deadband region; these fits are used to define a feedforward term in the PID engine speed controller to mitigate hysteresis effects. Green data points show steady states attained by accelerating from lower speeds to higher speeds while red data points show steady states attained by decelerating from higher speeds to lower speeds. Within a single speed mode hysteresis is prevalent but further amplified along the boarder between the semi-displacement and planing modes.

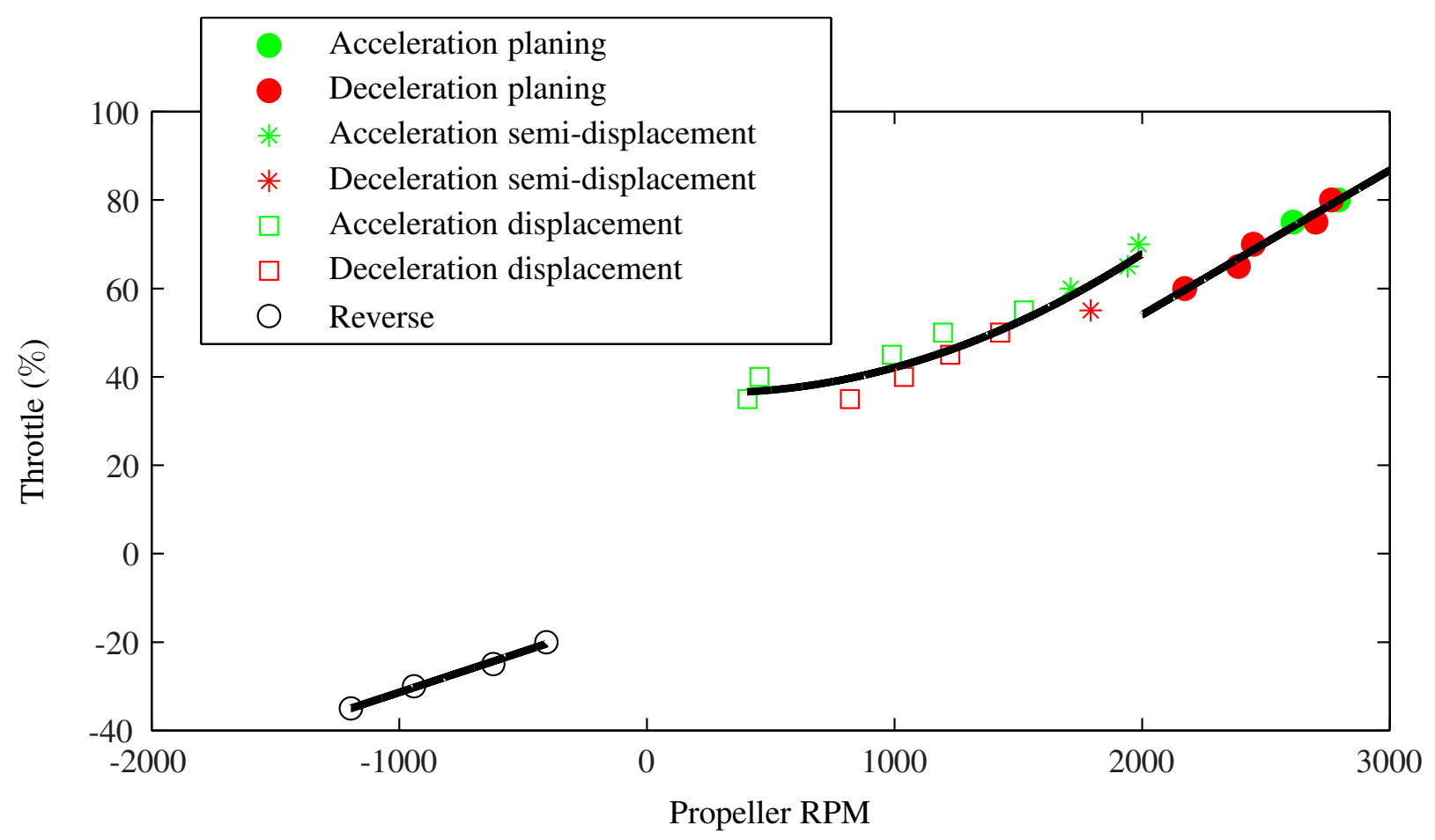

Figure 5.3: Steady state throttle fits

\subsubsection{Thruster Model}

The detailed steps required to extract $\tilde{c}$ and $\tilde{d}$ from (5.7), as introduced in Section 5.2.1, are as follows:

1. Define $\delta \tilde{T}=35 \%$ and $\delta \tilde{T}_{\mathrm{SS}}=0 \%$ and set $\delta s=0$ with the USV heading in a safe direction. 
2. Set throttle to $\delta T=\delta \tilde{T}_{\mathrm{ss}}$ and wait for steady state

3. Set throttle to $\delta T=\delta \tilde{T}$ and wait for steady state

4. If, $\delta \tilde{T}<=65 \%$ increment $\delta \tilde{T}$ by $5 \%$ and return to Step 2. Else, go to Step 5 .

5. If, $\delta \tilde{T}_{\mathrm{ss}}<=60 \%$ increment $\delta \tilde{T}_{\mathrm{ss}}$ by $5 \%$, set $\delta \tilde{T}=\delta \tilde{T}_{\mathrm{ss}}+5 \%$ and return to Step 2. Else, end the test sequence.

for identification in the displacement and semi-displacement region. For planing thruster identification the test sequence is as follows:

1. Define $\delta \tilde{T}=75 \%$ and $\delta \tilde{T}_{\mathrm{sS}}=70 \%$ and set $\delta s=0$ with the USV heading in a safe direction.

2. Set throttle to $\delta T=\delta \tilde{T}_{\mathrm{sS}}$ and wait for steady state

3. Set throttle to $\delta T=\delta \tilde{T}$ and wait for steady state

4. If, $\delta \tilde{T}<=95 \%$ increment $\delta \tilde{T}$ by $5 \%$ and return to Step 2. Else, go to Step 5 .

5. If, $\delta \tilde{T}_{\mathrm{sS}}<=90 \%$ increment $\delta \tilde{T}_{\mathrm{ss}}$ by $5 \%$, set $\delta \tilde{T}=\delta \tilde{T}_{\mathrm{sS}}+5 \%$ and return to Step 2. Else, end the test sequence.

Velocity, acceleration, and propeller speed are recorded during the execution of the steps to extract $\tilde{c}$ and $\tilde{d}$ in (5.7). Figure 5.4 shows a fit of $\tilde{c}$ and $\tilde{d}$. The thrust fit matches the data well, yielding parameter values $\tilde{c}=-1.42 \times 10^{-4}\left(\mathrm{~s}^{-1} \mathrm{RPM}^{-1}\right)$ and $\tilde{d}=8.96 \times 10^{-7}\left(\mathrm{~ms}^{-2} \mathrm{RPM}^{-2}\right)$. Note, however, that experimental data were unavailable at high RPM and low speed or at low RPM and high speed.

\subsubsection{Speed and Steering Dynamic Parameters}

The nonlinear speed model with identified thrust parameters only contains two additional parameters to identify, which are used to constrain steady state speed behavior. A regression is performed to find these drag parameters by utilizing the constraint that drag must balance thrust for all steady speeds. Figures 5.5 and 5.6 show a comparison of the estimated acceleration due to drag and thrust for displacement and planing 


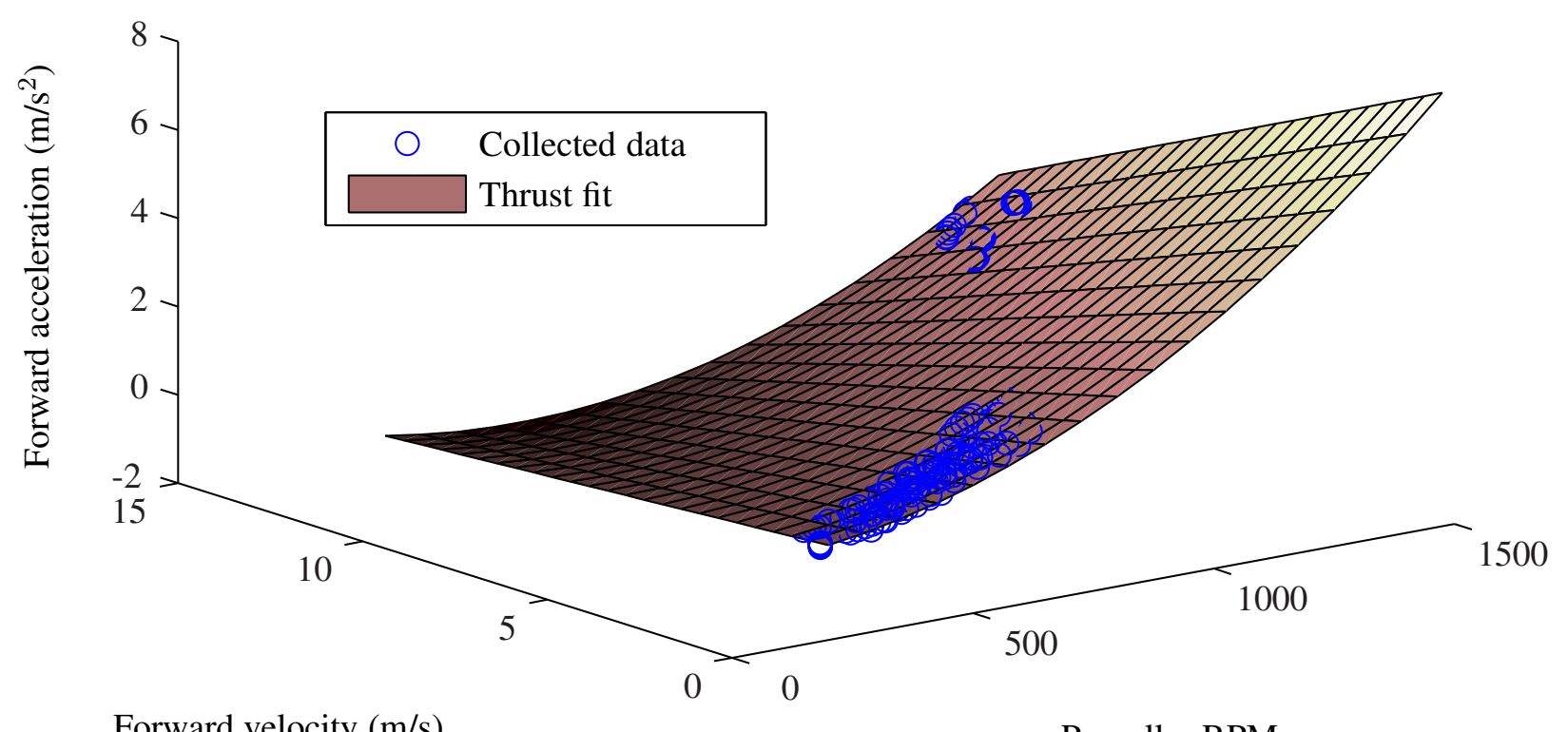

Forward velocity $(\mathrm{m} / \mathrm{s})$

Propeller RPM

Figure 5.4: Thrust parameter fitting

regions respectively. This fit between thrust and drag seems to match well though validation plots will show that these small discrepancies lead to large steady state speed errors.

Figures 5.7, 5.8, and 5.9 show actual acceleration terms compared to acceleration estimated by the simplified dynamic models containing identified parameters. The difference between these acceleration terms is minimized in the least squares sense by finding model parameters using a "batch" regression. The estimated acceleration terms are computed using the actual smoothed input and state data combined with a model structure containing identified parameters. Recall that the actual acceleration terms are found as a byproduct of the Savitzky-Golay smoothing filter.

Figures 5.10, 5.11, and 5.12 show representative time histories (actual and simulated) of $u, r$, and $\beta$ for 


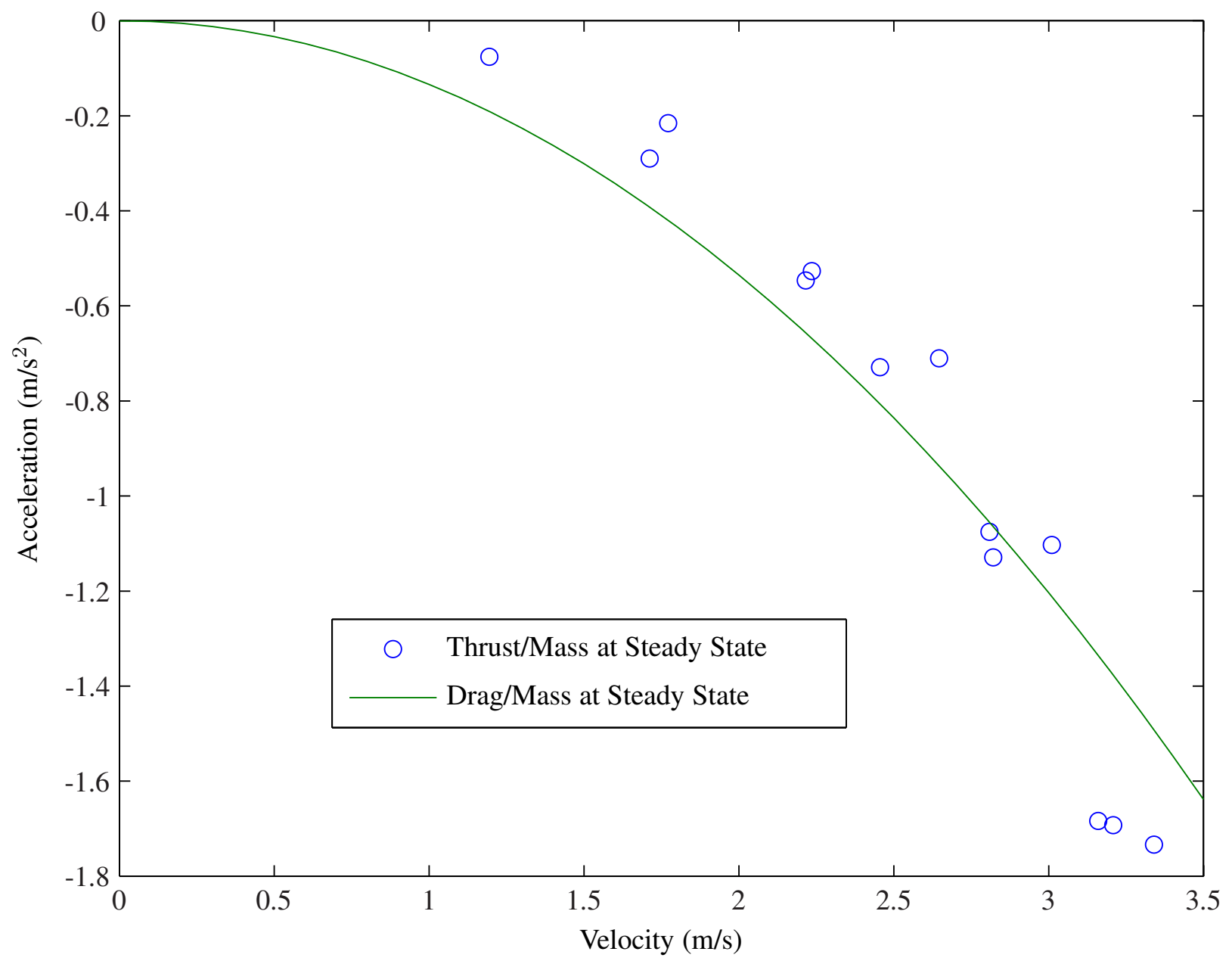

Figure 5.5: Estimated thrust and drag for the nonlinear speed model in the displacement region 


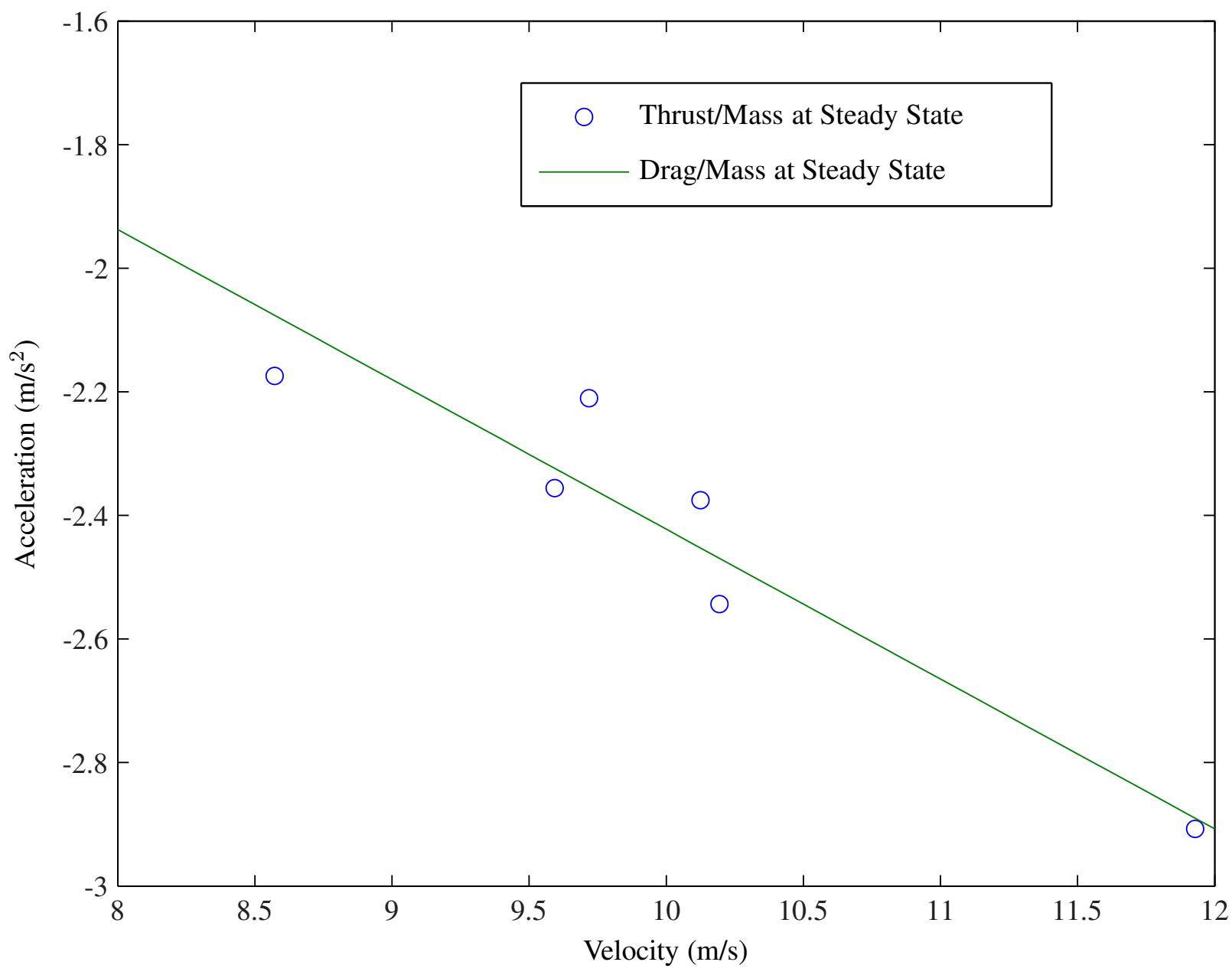

Figure 5.6: Estimated thrust and drag for the nonlinear speed model in the planing region 


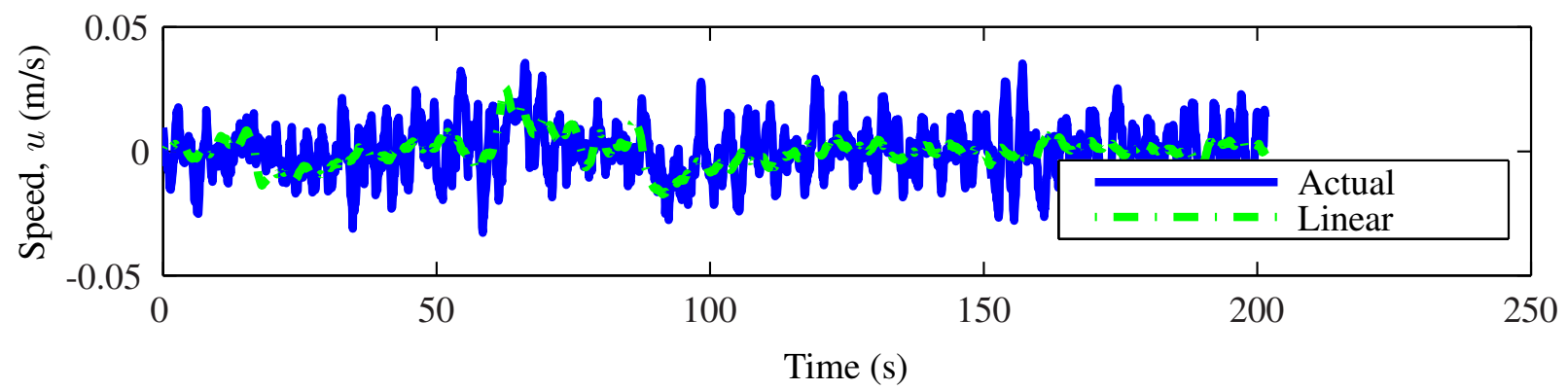

(a) $1.1(\mathrm{~m} / \mathrm{s})$

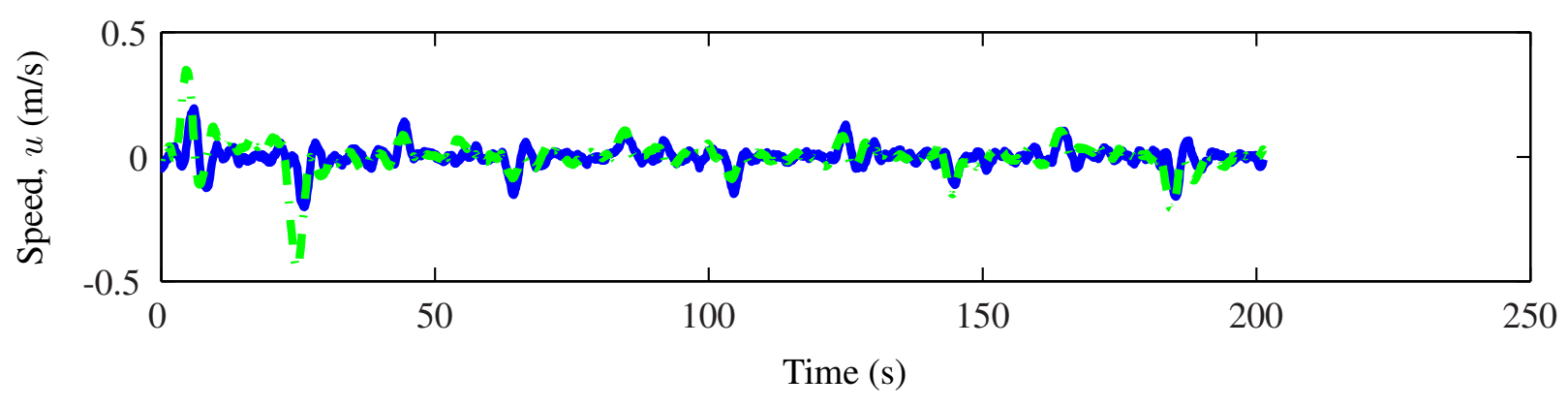

(b) $3.0(\mathrm{~m} / \mathrm{s})$

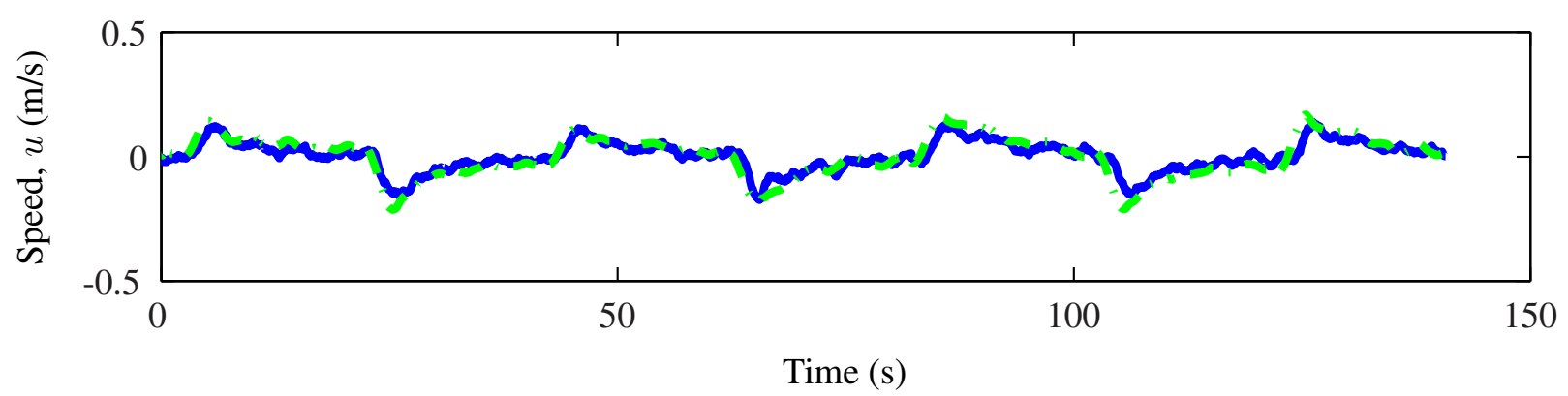

(c) $7.6(\mathrm{~m} / \mathrm{s})$

Figure 5.7: Actual and estimated forward acceleration for a range of forward speeds 


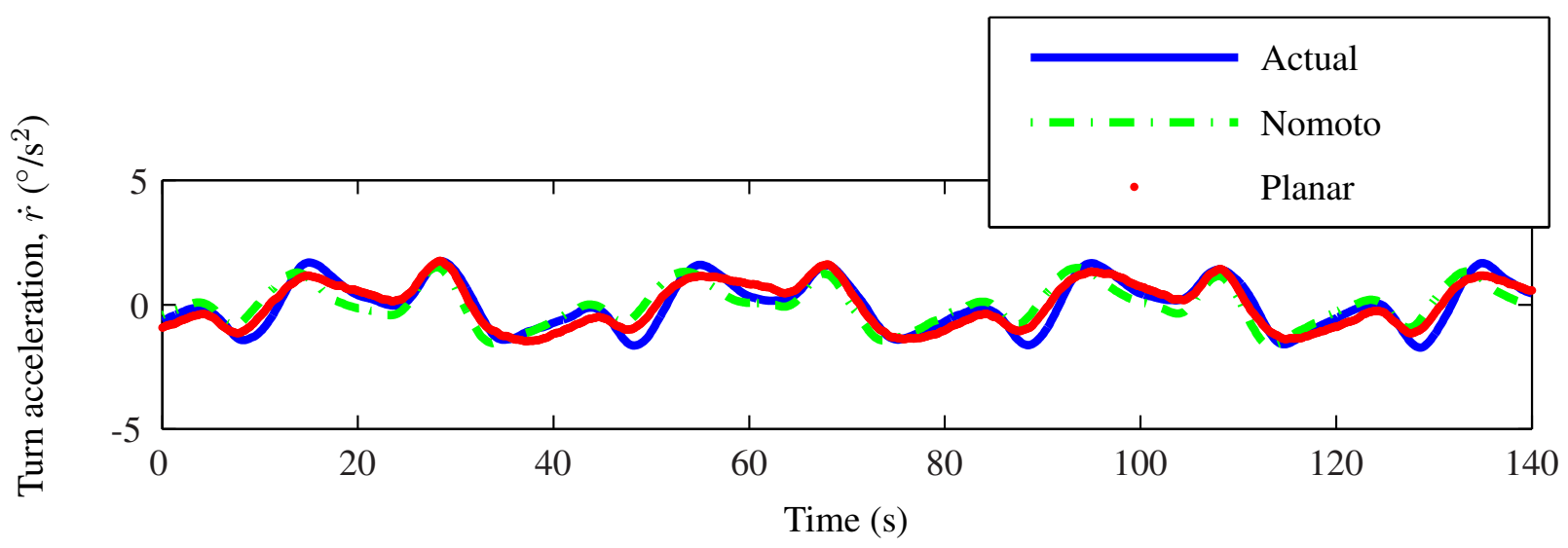

(a) $1.1(\mathrm{~m} / \mathrm{s})$

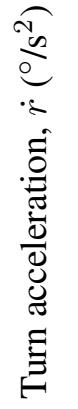

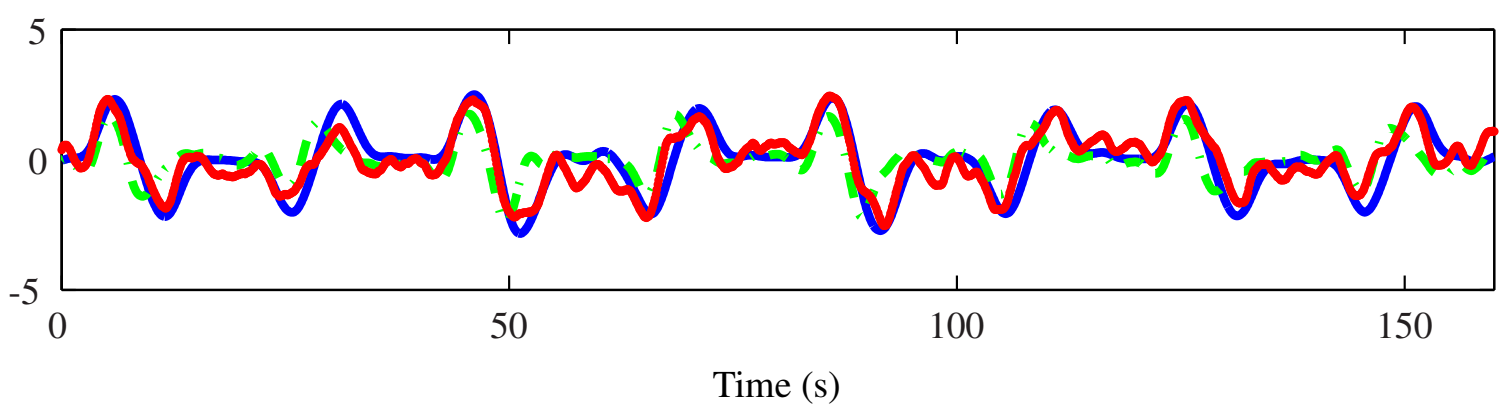

(b) $3.0(\mathrm{~m} / \mathrm{s})$

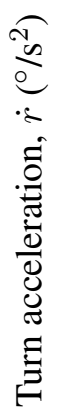

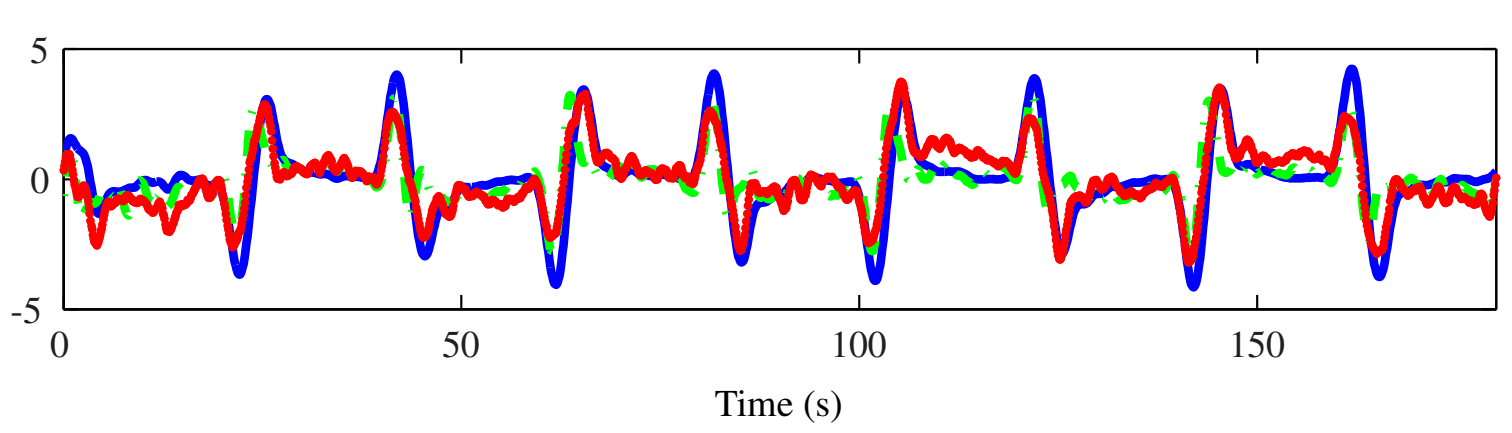

(c) $7.6(\mathrm{~m} / \mathrm{s})$

Figure 5.8: Actual and estimated turn acceleration for a range of forward speeds 


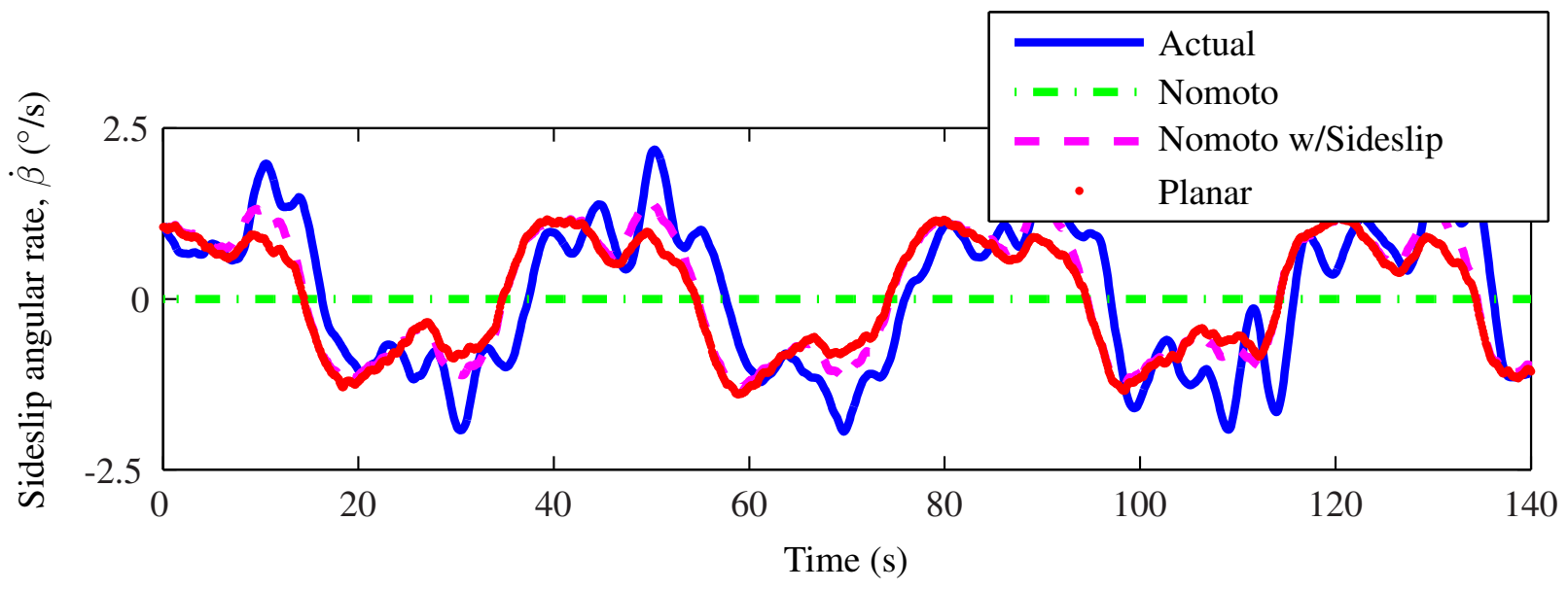

(a) $1.1(\mathrm{~m} / \mathrm{s})$

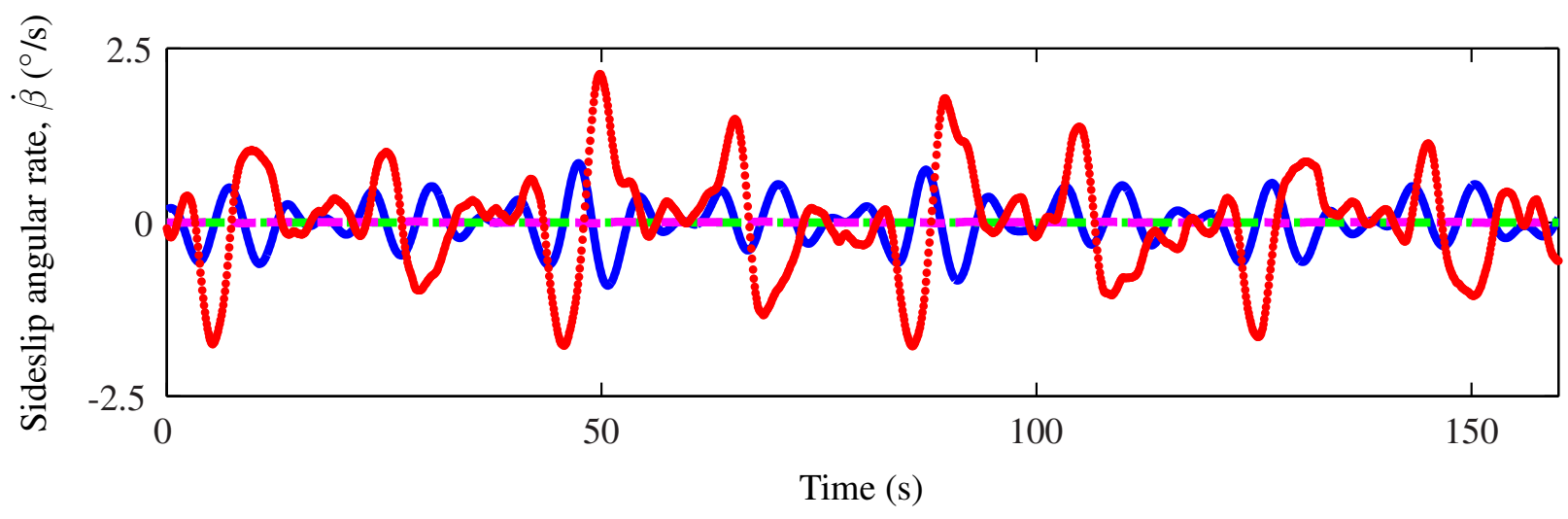

(b) $3.0(\mathrm{~m} / \mathrm{s})$

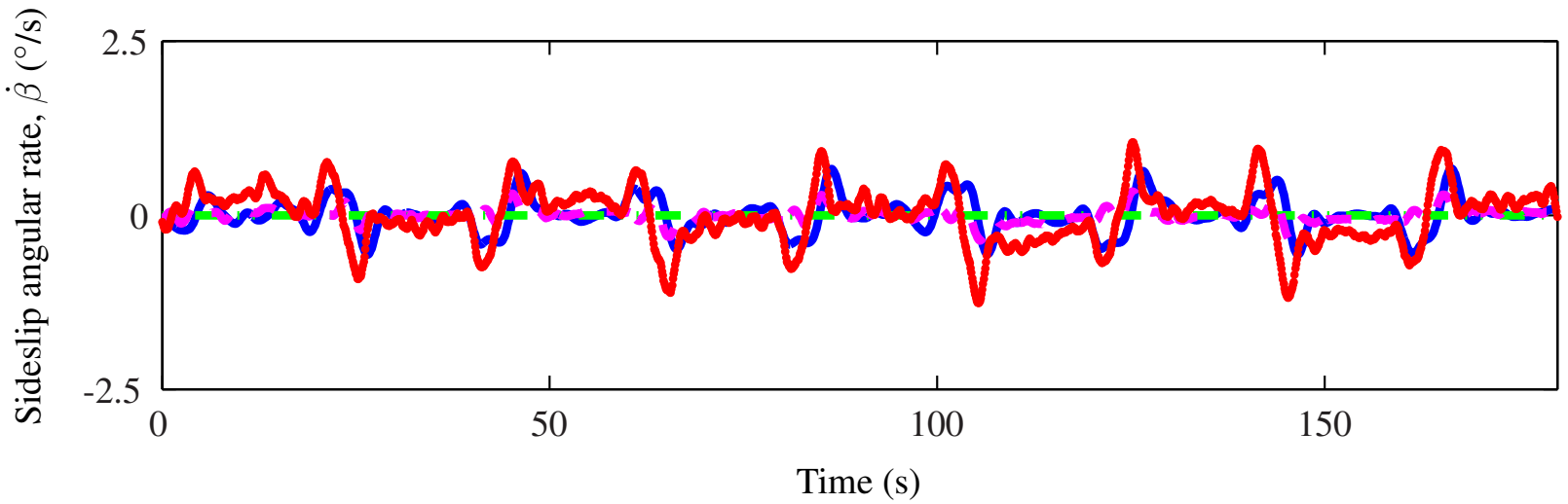

(c) $7.6(\mathrm{~m} / \mathrm{s})$

Figure 5.9: Actual vs. estimated sideslip angular rate for a range of forward speeds 
the feedback controlled Ribcraft USV in response to commanded square waves in heading and speed. These plots use validation data sets, obtained independently of the data used for model identification. Examining Figure 5.10, it is clear that the first order linear speed model identified for this displacement speed range captures the behavior of the vehicle in surge while the nonlinear speed model fails to converge to correct steady state velocities. A single set of parameters for the nonlinear speed model, identified for each speed regime using steady state behavior (shown in Figures 5.5 and 5.6), poorly estimates speed behavior, which indicates that quadratic and linear drag terms do not adequately model the vessels speed dynamics. For the data shown in the Figures 5.11 and 5.12, the Nomoto model with sideslip and the linear model best approximate the true course angle history when the boat is moving at low speeds. This observation illustrates the need to account for sideslip dynamics at low speeds, when the vessel is operating in displacement mode.

Table 5.2 shows parameter identification cost function values for identified models of the Ribcraft USV steering dynamics. These values were computed using the same validation data sets used to make Figures 5.10, 5.11, and 5.12. Time histories of estimated turn rate and sideslip angle are calculated using steering model parameters and actual thrust angle time histories taken from validation data. The cost function

$$
J=\sqrt{\frac{1}{t_{\text {total }}} \sum_{i=1}^{N}\left(\beta_{\text {est }}(i)-\beta_{\text {val }}(i)\right)^{2}}+\sqrt{\frac{1}{t_{\text {total }}} \sum_{i=1}^{N}\left(r_{\text {est }}(i)-r_{\text {val }}(i)\right)^{2}}
$$

provides a comparison between estimated turn rate and sideslip and actual turn rate and sideslip (from the validation data). The value $t_{\text {total }}$ is the total time of the time histories, states with the "val" subscript denote actual validation data, and states with the "est" subscript denote states estimated with steering model parameters. Note that asterisks in Table 5.2 indicate that the given parameters yield an unstable system.

Since steady state sideslip angle decreases to near zero for some displacement and semi-displacement speed regions, sideslip angle may not be sufficiently excited to overcome signal and wave noise in these speed regions. Noiseless parameter identification would give time constants that are negative but very close to zero, but the parameter identification method, using the derivative of state containing wave and signal noise, does not guarantee "well-behaved" models. 


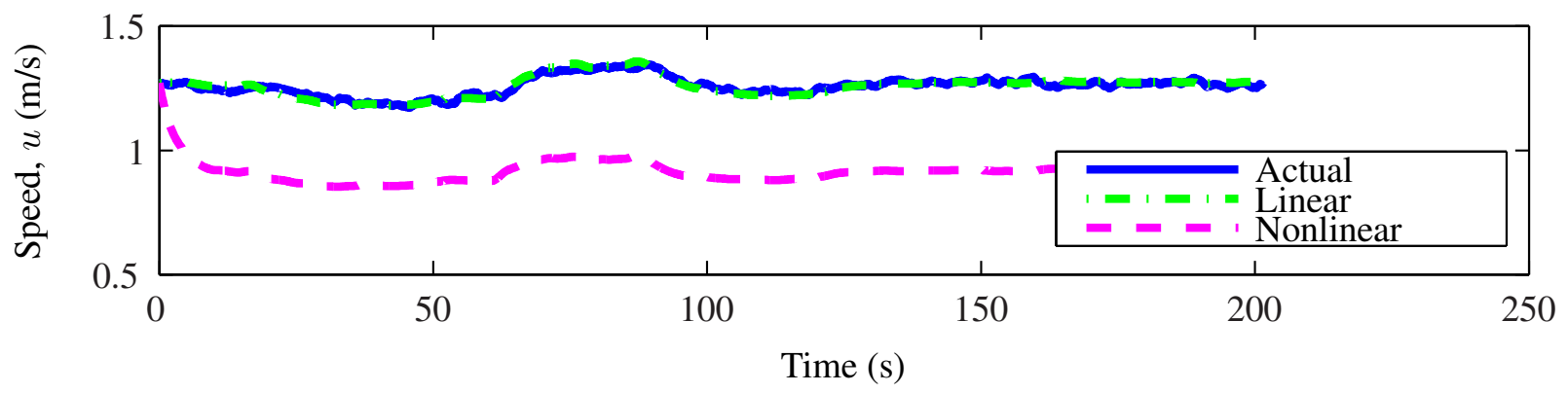

(a) $1.1(\mathrm{~m} / \mathrm{s})$

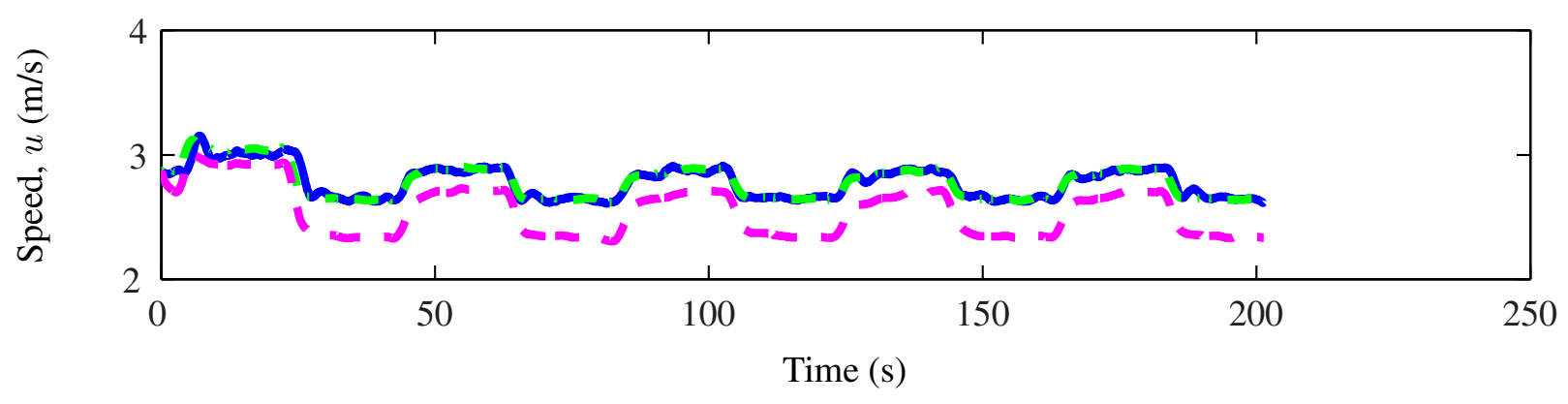

(b) $3.0(\mathrm{~m} / \mathrm{s})$

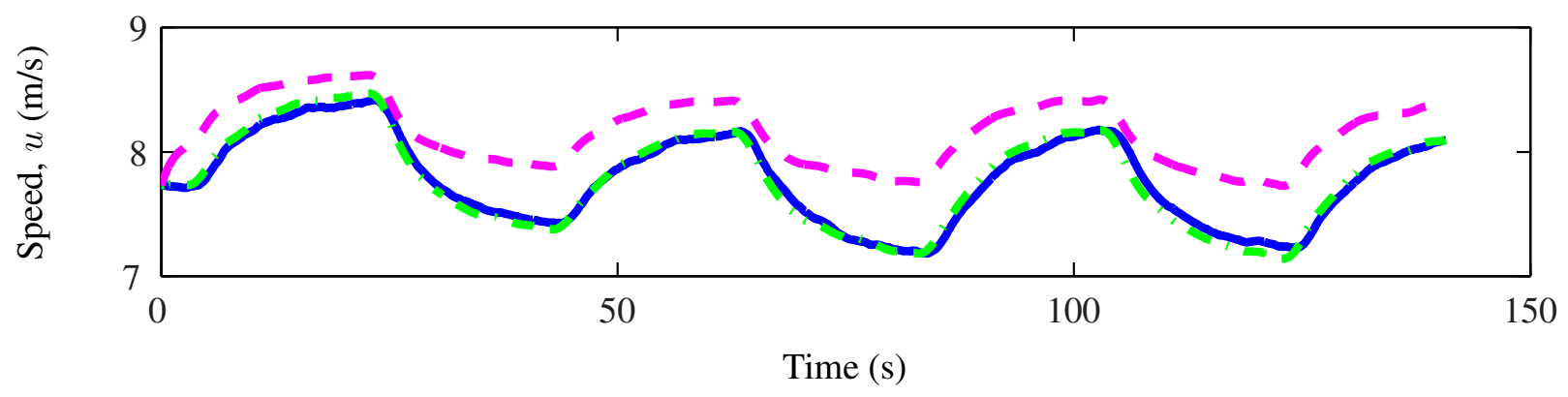

(c) $7.6(\mathrm{~m} / \mathrm{s})$

Figure 5.10: Measured forward speed vs. simulated forward speed for a range of forward speeds 


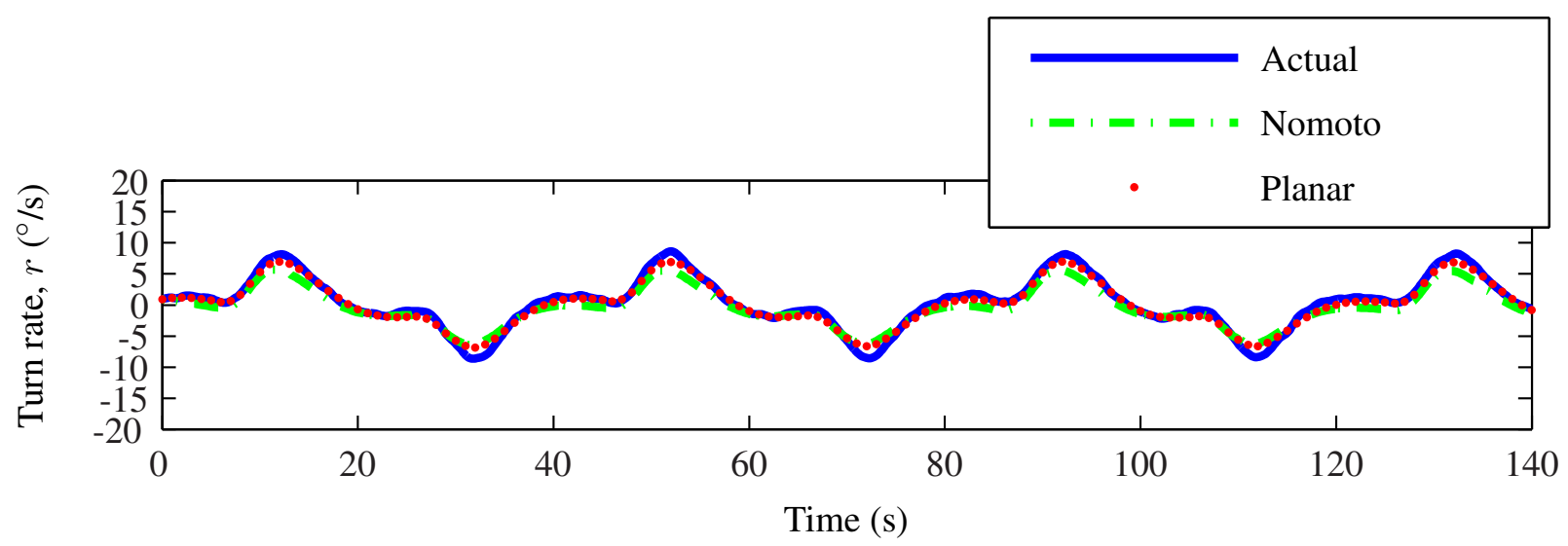

(a) $1.1(\mathrm{~m} / \mathrm{s})$

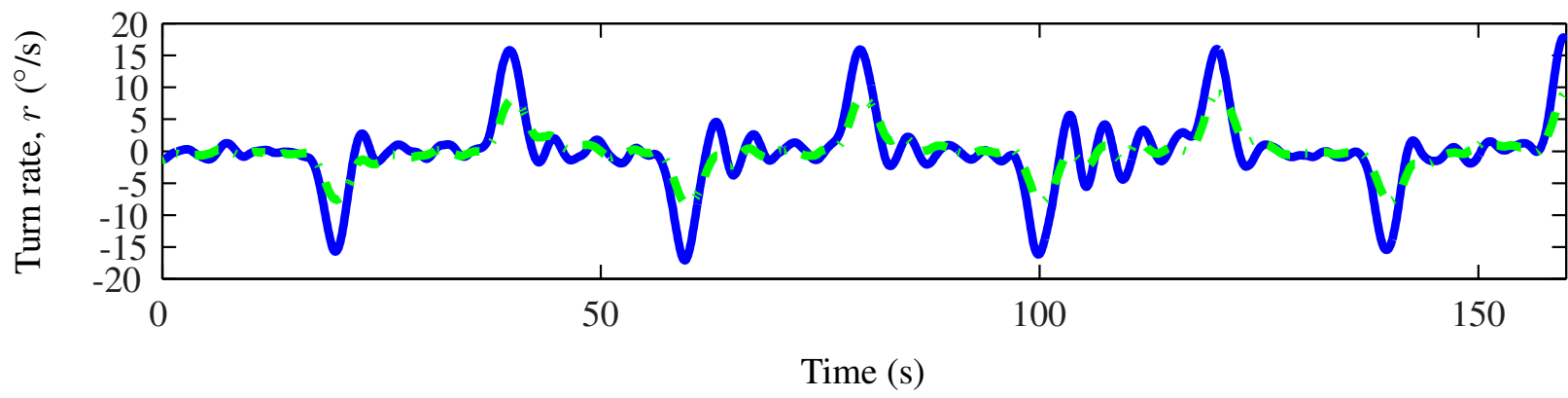

(b) $3.0(\mathrm{~m} / \mathrm{s})$

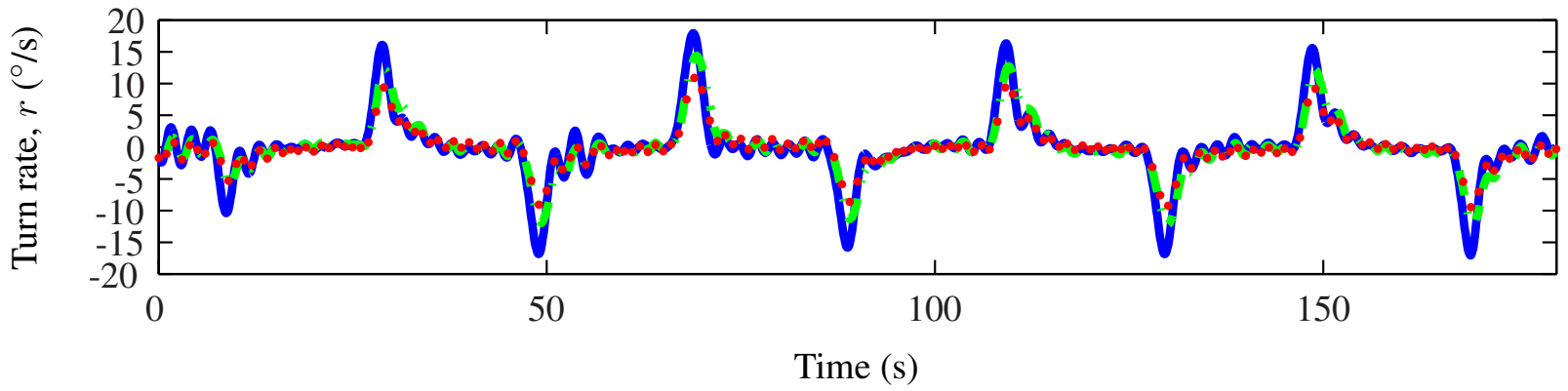

(c) $7.6(\mathrm{~m} / \mathrm{s})$

Figure 5.11: Actual and simulated turn rate time histories for a range of forward speeds 


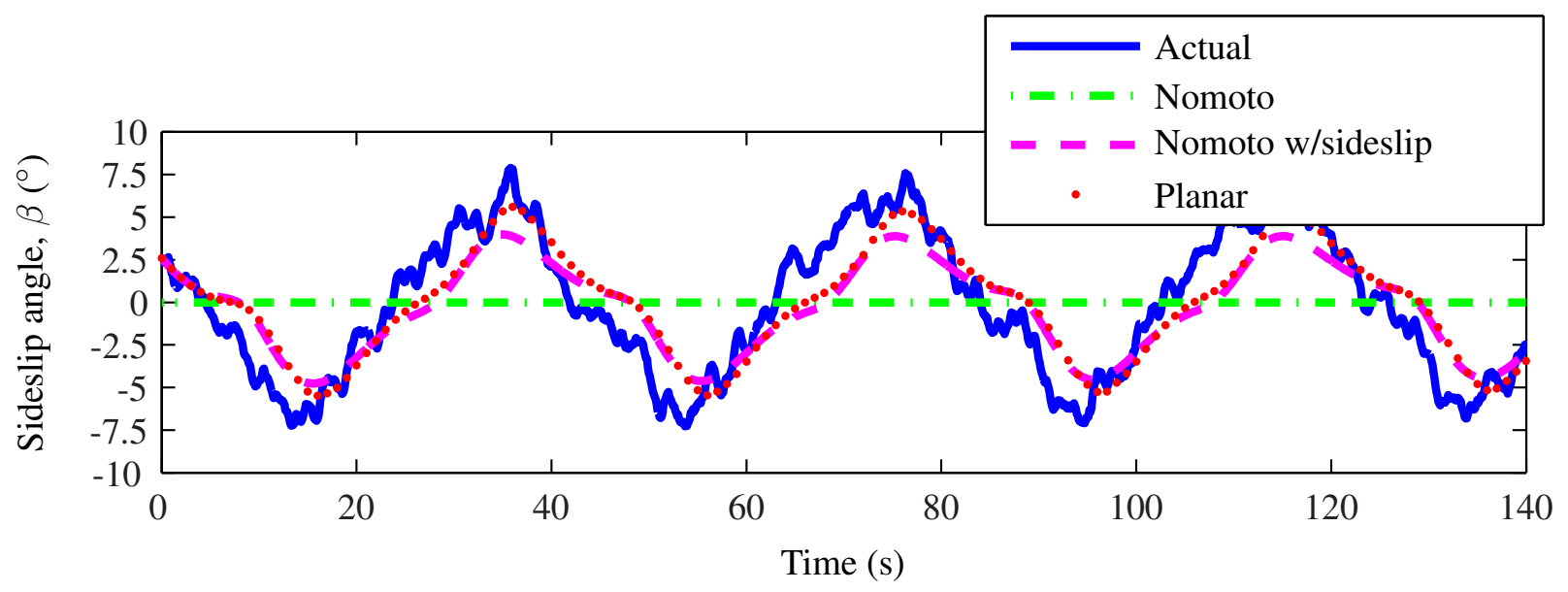

(a) $1.1(\mathrm{~m} / \mathrm{s})$

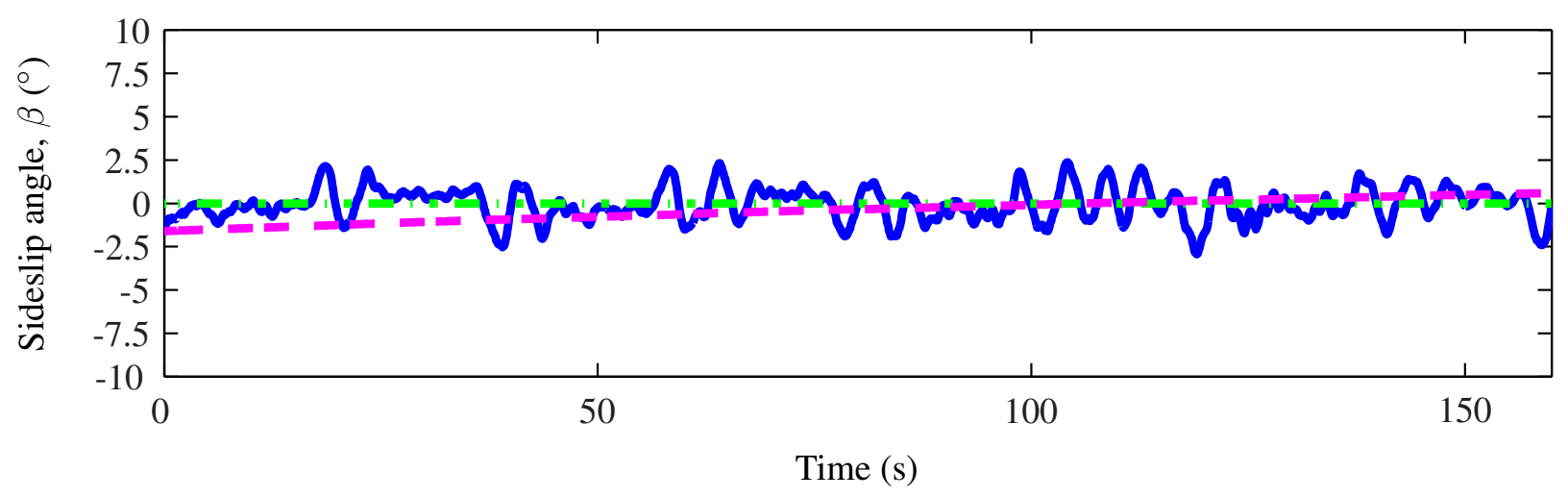

(b) $3.0(\mathrm{~m} / \mathrm{s})$

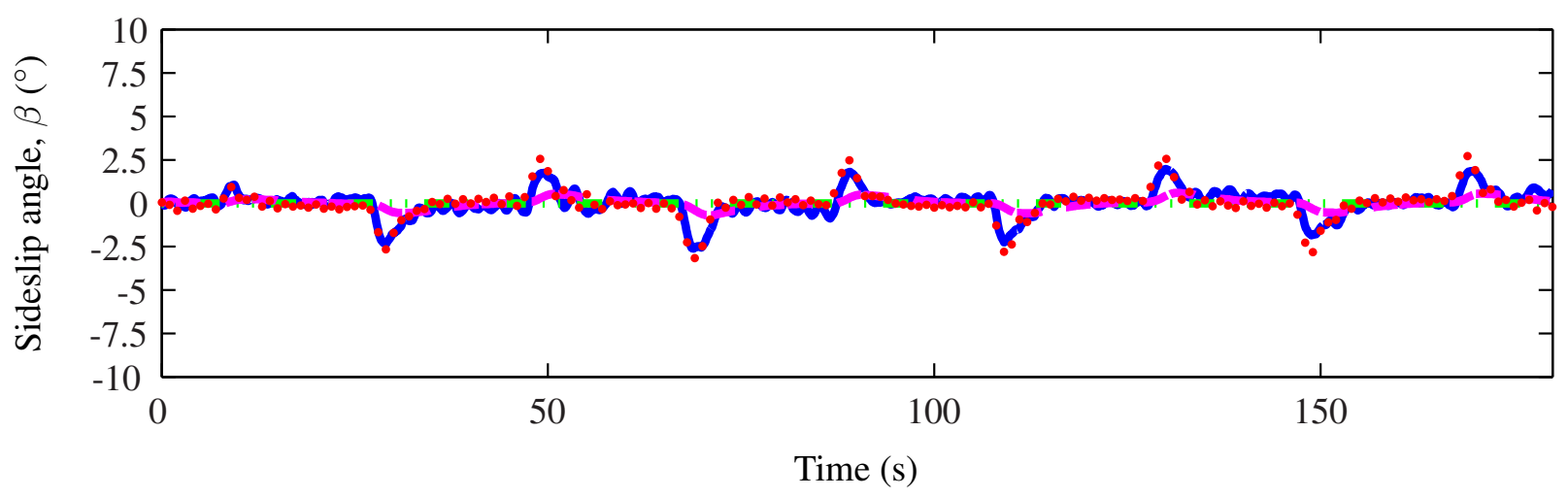

(c) $7.6(\mathrm{~m} / \mathrm{s})$

Figure 5.12: Actual vs. simulated sideslip angle time histories for a range of forward speeds 
Table 5.2: Steering model cost function values.

\begin{tabular}{c|ccc} 
Forward Speed, (m/s) & Nomoto & Nomoto w/ Side Slip & Linear \\
\hline 1.1 & 5.5 & 3.6 & 2.7 \\
1.5 & 2.5 & 1.9 & 2.3 \\
2.6 & 1.9 & 1.9 & $*$ \\
3.0 & 1.4 & 1.4 & $*$ \\
3.2 & 0.8 & 0.9 & $*$ \\
4.0 & 0.4 & 0.4 & 0.7 \\
7.6 & 0.3 & 0.3 & 0.3 \\
9.2 & 0.5 & 0.5 & 0.4
\end{tabular}

It is unsurprising that the linear steering model performs well at low speeds, since this model has the most parameters. The Nomoto-with-sideslip model performs comparably, however, and requires fewer parameters. Because the sideslip angle remains quite small during maneuvers at higher speeds one may justifiably adopt the Nomoto steering model in this speed region. Consequently a speed-scheduled Nomoto-withsideslip model (4.24) is used to describe vessel motion for generating and tracking trajectories. The model parameters that describe sideslip dynamics are non-zero at low speed, but are set equal to zero at higher speeds, effectively constraining the sideslip angle to remain zero.

Table 5.3 shows the parameter values obtained for the linear steering model (4.21). Table 5.4 shows the parameter values for the Nomoto model with sideslip (4.24). Table 5.5 shows the parameter values for the Nomoto model (4.22). Table 5.6 shows the parameter values obtained for the linear speed model (4.18). Note that $\tilde{T}=\frac{T}{m-X_{u}}=b_{u} T$ is used in system identification, so $b_{u}$ is not needed for the linear speed model. In these tables, $a_{*}$ and $b_{*}$ refer to the elements $*$ in the state matrix $\boldsymbol{A}$ and input matrix $\boldsymbol{B}$ respectively. If $a$ and $b$ appear without a subscript, then the state and input matrices are scalars. 


\subsubsection{Special Considerations: Sternward Steering Bifurcation Behavior}

Field trials used for forward motion parameter identification must be adjusted for the Ribcraft USV's unique sternward system identification while the analysis methods remain the same. The steady state turn rate behavior is the most prominent example of this difference between forward and sternward motion analysis. To identify steady state sternward behavior the following steps were performed:

1. Saturate $\delta s$ to the left (negative) with the USV heading in a safe direction at idle speed.

2. Set throttle to a set negative value. Allow the speed to reach steady-state.

a) Increment $\delta s$ by a fixed amount (e.g., 7.5 $)$.

b) Wait for the USV to complete two circular paths.

c) If the turn rate does not change sign, return to Step 2(a). Else, continue

d) If current turn rate is negative, saturate $\delta s$ to the right (positive) and return to Step 2 incrementing by negative thrust angle values. Else, end the test sequence

Note that the bifurcated sternward dynamics requires finding steady state behavior for both stable turn regions, embodied in the first step of saturating the thrust angle to the left, which forces the vessel into the left equilibrium. The thrust angle is incremented in this stable left turn region until falling into the right turn equilibrium. This procedure is then repeated for the right turn equilibrium. Steady state sternward behavior can be seen in Figure 5.13. Quadratic fits map these equilibrium turn rate regions to thrust angle

Table 5.3: Linear steering model parameter values (using linear fit of steady-state gains).

\begin{tabular}{c|cccccccc} 
Forward Speed, $(\mathrm{m} / \mathrm{s})$ & $a_{11}$ & $a_{12}$ & $a_{21}$ & $a_{22}$ & $b_{1}$ & $b_{1_{\text {bias }}}$ & $b_{2}$ & $b_{2_{\text {bias }}}$ \\
\hline 1.1 & -0.14 & -0.004 & 4.62 & -0.19 & -0.0009 & 0.0017 & -0.91 & 0.20 \\
1.5 & -0.29 & 0.002 & 19.53 & -0.15 & 0.0116 & -0.0031 & -0.79 & 0.21 \\
2.6 & -0.31 & 0.020 & 14.38 & -0.52 & 0.0191 & -0.0289 & -0.56 & 0.57 \\
3.0 & -0.29 & 0.032 & 17.28 & -0.92 & 0.0194 & -0.0624 & -0.58 & 1.59 \\
3.2 & -0.08 & 0.031 & 17.60 & -1.14 & 0.0145 & -0.0576 & -0.54 & 1.32 \\
4.0 & -0.46 & 0.066 & -8.71 & -0.99 & 0.0228 & -0.0969 & -0.37 & 2.71 \\
7.6 & -0.81 & 0.034 & 18.59 & -0.89 & 0.0159 & 0.0323 & -0.41 & -0.48 \\
9.2 & -0.42 & 0.013 & 11.99 & -0.39 & 0.0068 & 0.0222 & -0.20 & -0.61
\end{tabular}


Table 5.4: Nomoto-with-sideslip steering model parameter values.

\begin{tabular}{c|cccccc} 
Forward Speed, $(\mathrm{m} / \mathrm{s})$ & $a_{\beta}$ & $b_{\beta}$ & $b_{\beta_{\text {bias }}}$ & $a_{r}$ & $b_{r}$ & $b_{r_{\text {bias }}}$ \\
\hline 1.1 & -0.140 & -0.20 & 0.061 & -0.34 & -1.06 & 0.29 \\
1.5 & -0.135 & -0.10 & 0.008 & -0.40 & -1.11 & 0.77 \\
2.6 & 0.035 & -0.01 & 0.042 & -0.39 & -0.33 & 0.78 \\
3.0 & -0.004 & -0.00 & 0.003 & -0.52 & -0.30 & 1.10 \\
3.2 & -0.001 & 0.00 & 0.001 & -0.69 & -0.33 & 1.40 \\
4.0 & -0.175 & 0.01 & 0.205 & -1.29 & -0.46 & 2.62 \\
7.6 & -0.275 & -0.03 & 0.337 & -1.08 & -0.39 & 2.47 \\
9.2 & -0.203 & -0.02 & 0.226 & -0.29 & -0.10 & 0.65
\end{tabular}

values well with $r_{\mathrm{ss}}=-0.0032 \delta s^{2}-0.4428 \delta s+11.99525$ and $r_{\mathrm{ss}}=0.0072 \delta s^{2}-0.5684 \delta s-6.77138$ corresponding to mappings for the left and right equilibrium region respectively where $r_{\mathrm{sS}}$ is measured in $\mathrm{deg} / \mathrm{s}$ and $\delta s$ is measured in deg. Taking reverse speed and thrust angle as bifurcation parameters, one finds a pitchfork bifurcation at zero thrust angle that degenerates into a saddle-node bifurcation when the thrust angle is offset.

Thrust identification is constrained to only finding the quadratic thrust term. The limited sternward speed envelope prevents field trials with thrust step inputs starting from a steady speed. However, since speed remain low, thrust is estimated well by without a thrust decay term. Field trials for sternward thrust identification involve commanding step inputs in throttle starting from rest. A thrust parameter of $\tilde{d}=7.31 \times 10^{-8}$ $\left(\mathrm{ms}^{-2} \mathrm{RPM}^{-2}\right)$ fits the data shown in Figure 5.14.

Table 5.5: Nomoto steering model parameter values.

\begin{tabular}{c|ccc} 
Forward Speed, $(\mathrm{m} / \mathrm{s})$ & $a_{r}$ & $b_{r}$ & $b_{r_{\text {bias }}}$ \\
\hline 1.1 & -0.34 & -1.06 & 0.29 \\
1.5 & -0.40 & -1.11 & 0.77 \\
2.6 & -0.39 & -0.33 & 0.78 \\
3.0 & -0.52 & -0.30 & 1.10 \\
3.2 & -0.69 & -0.33 & 1.40 \\
4.0 & -1.29 & -0.46 & 2.62 \\
7.6 & -1.08 & -0.39 & 2.47 \\
9.2 & -0.29 & -0.10 & 0.65
\end{tabular}


Table 5.6: First order linear speed model parameter values.

\begin{tabular}{c|cc} 
Forward Speed, $(\mathrm{m} / \mathrm{s})$ & $a_{u}$ & $b_{u_{\text {bias }}}$ \\
\hline 1.1 & -0.12 & 0.07 \\
1.5 & -0.31 & 0.33 \\
2.6 & -0.58 & 0.85 \\
3.0 & -1.09 & 2.19 \\
3.2 & -1.00 & 1.75 \\
4.0 & -0.17 & -1.12 \\
7.6 & -0.06 & -1.65 \\
9.2 & -0.10 & -1.31
\end{tabular}

Parameter identification for both the nonlinear and linear speed models simply requires steady state data (recall the forward nonlinear speed model uses the same procedure). For the linear speed model to elicit zero steady state velocity for zero propeller speed, its biasing term must be zero, leaving only one parameter, which must be utilized to identify steady state motion. The nonlinear and linear speed models with identified parameters for sternward motion are

$$
\dot{u}=-0.14 u|u|+7.01 \times 10^{-8} \delta n|\delta n|
$$

and

$$
\dot{u}=-0.19 u+7.01 \times^{-8} \delta n|\delta n|
$$

respectively. Figure 5.15 shows a comparison of the estimated acceleration due to drag and thrust for the sternward speed region.

Stable left and right turn steering models separated by an unstable steering model situated at the zero turn rate condition are used to estimate the vehicle's bifurcated steering dynamics. By commanding either positive or negative sets of square waves in turn rate, instead of heading, sternward dynamics are excited for only one turn rate region at a time, ensuring that only one model is exercised by the experiment. Additionally commanding closed-loop control about zero turn rate allows for identification of steering parameters about the unstable equilibrium. Including the steady state mapping shown in Figure 5.13 into the standard turn rate PID controller allows for sufficient turn rate control for parameter identification. 


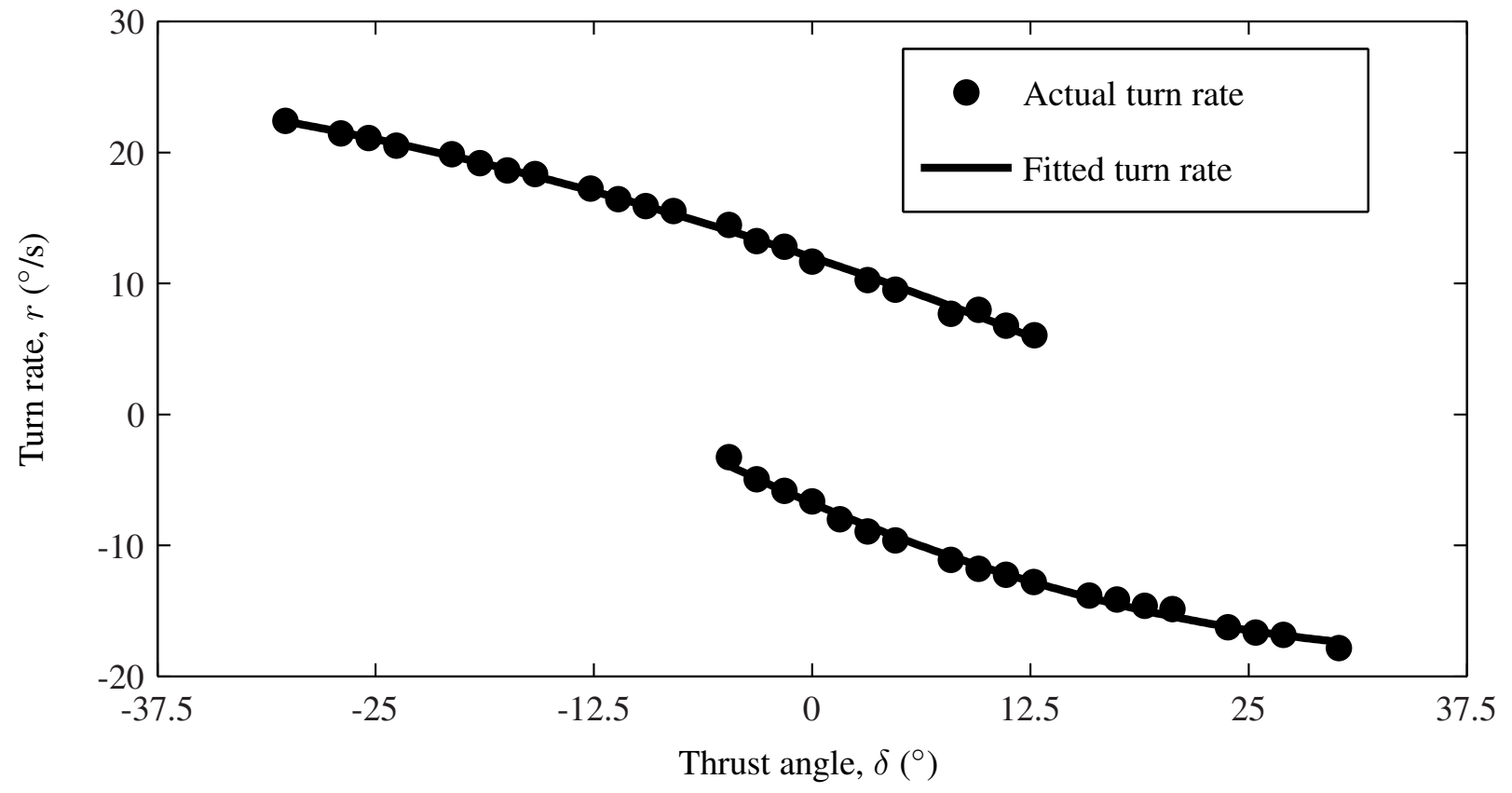

Figure 5.13: Steady state backwards turn rate fit

Figures 5.16 and 5.17 show actual acceleration terms compared to acceleration estimated by the simplified dynamic models. The difference between these acceleration terms is minimized in the least squares sense by finding model parameters using the same "batch" regression used for forward motion. The estimated acceleration terms are computed using the actual smoothed input and state data combined with a model structure containing identified parameters. Note that, unlike forward parameter identification, parameters are scheduled by regions of turn rate rather than speed. Also, the unstable model does not include steady state constraints.

Figures 5.18 and 5.19 show representative time histories (actual and simulated) of $r$ and $\beta$ for the feedback controlled Ribcraft USV in response to commanded square waves in positive, negative, and zero turn rate. These plots use validation data sets, obtained independently of the data used for model identification. Though the unstable zero turn rate validation plot may look featureless, the simulation generating the plot uses actual open loop thrust angle measurements; not closed-loop control. The closed-loop thrust angle 


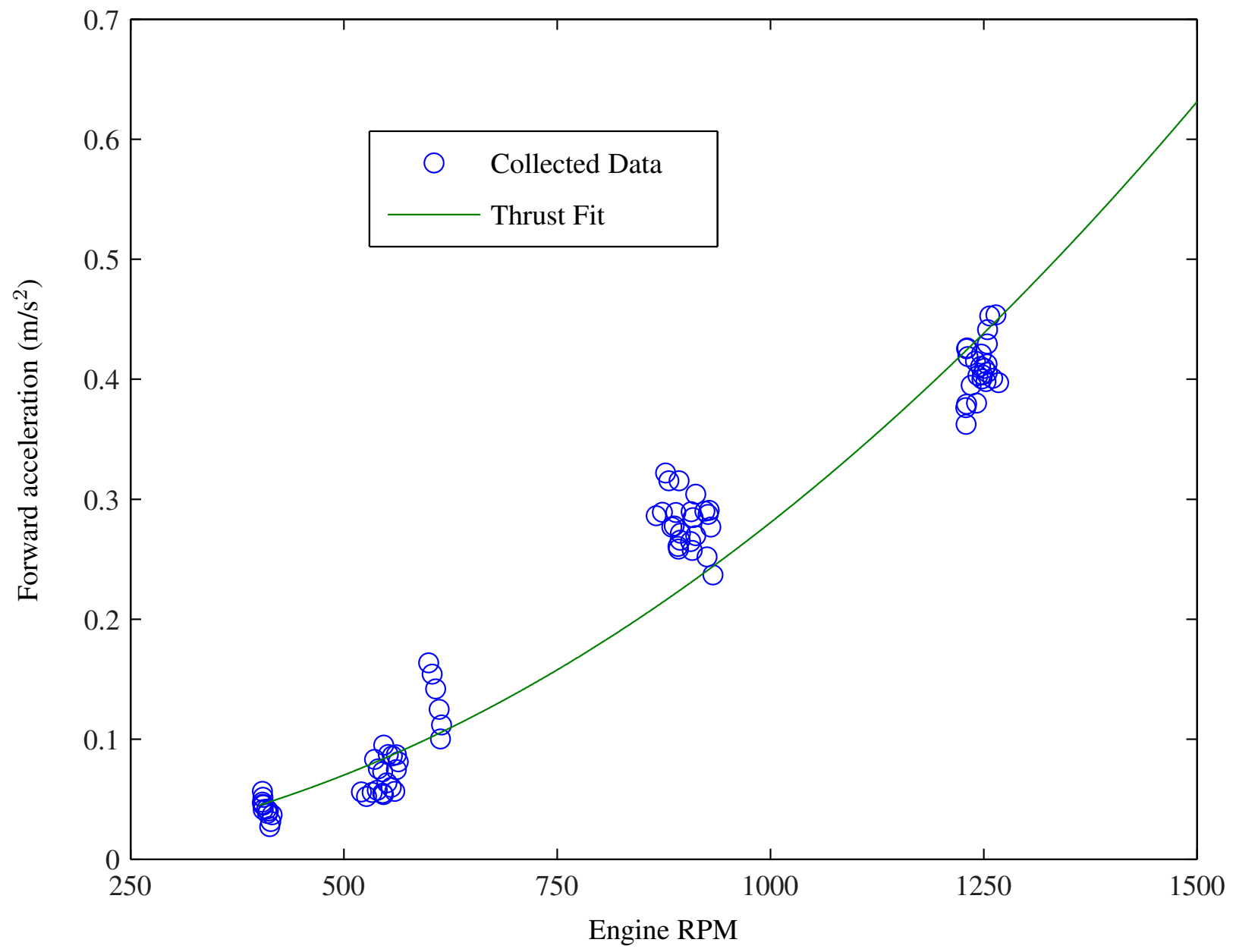

Figure 5.14: Thrust fit for sternward motion 


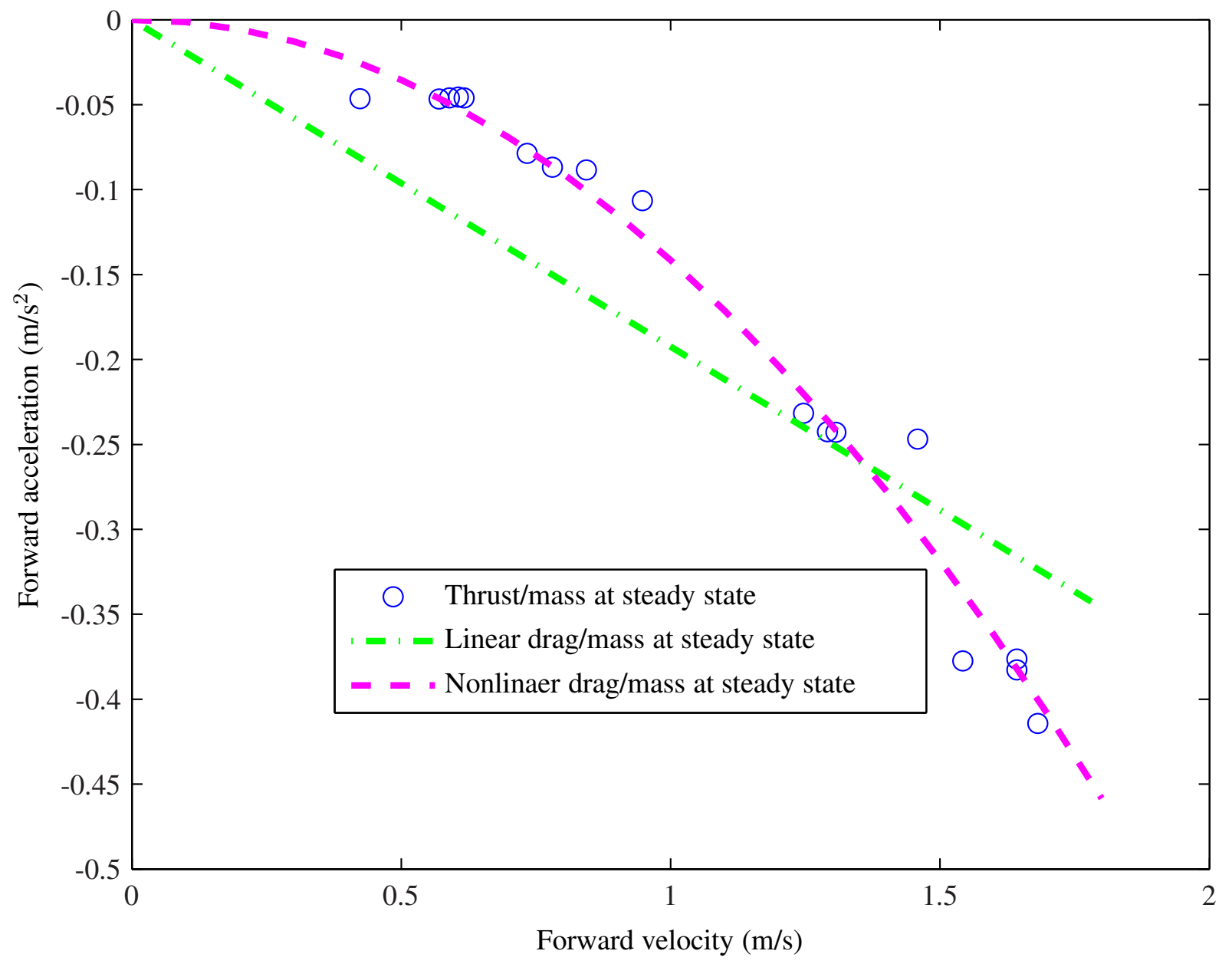

Figure 5.15: Estimated thrust and drag for the linear and nonlinear speed models for sternward motion 


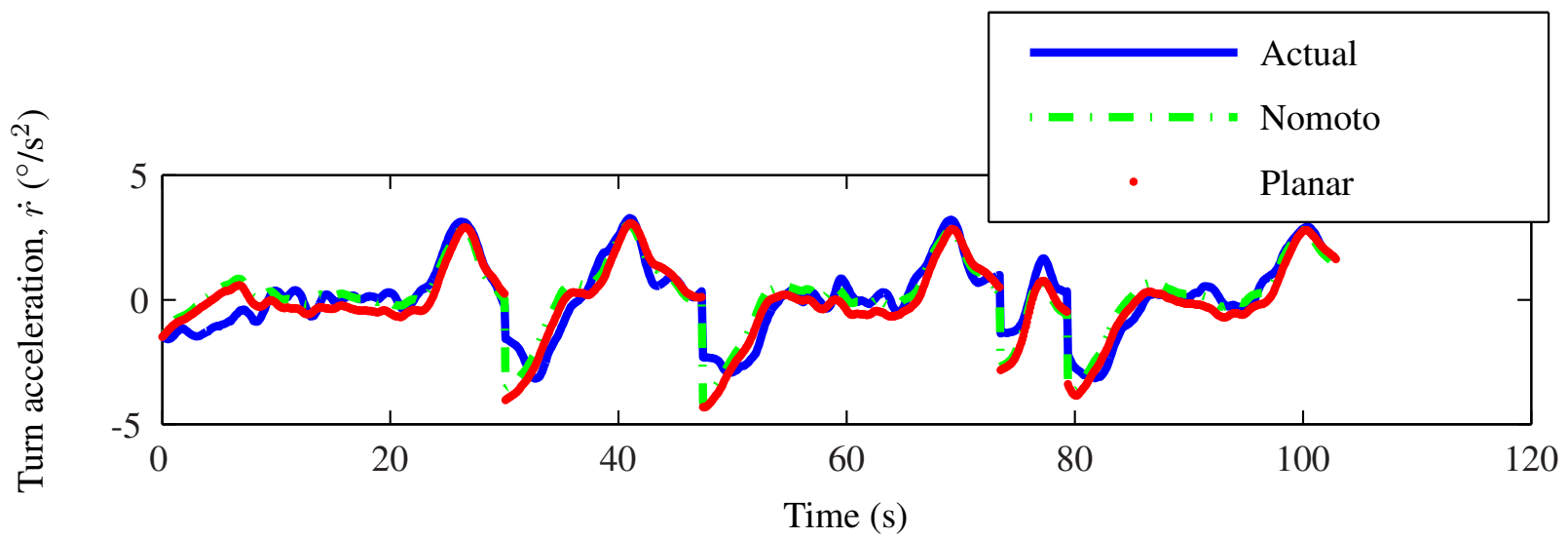

(a) positive turn rate

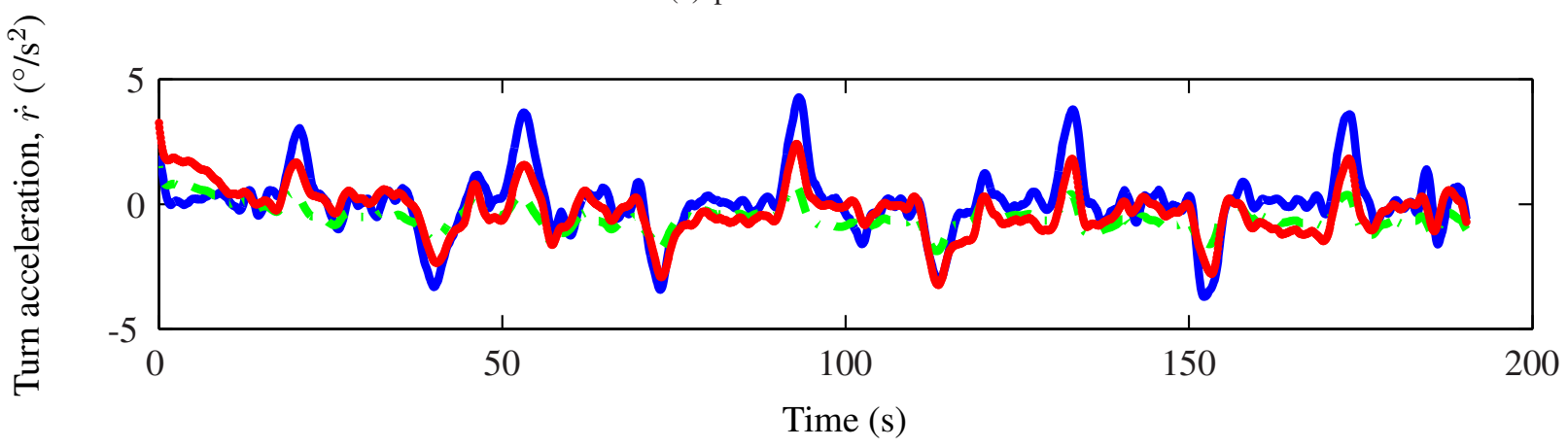

(b) negative turn rate

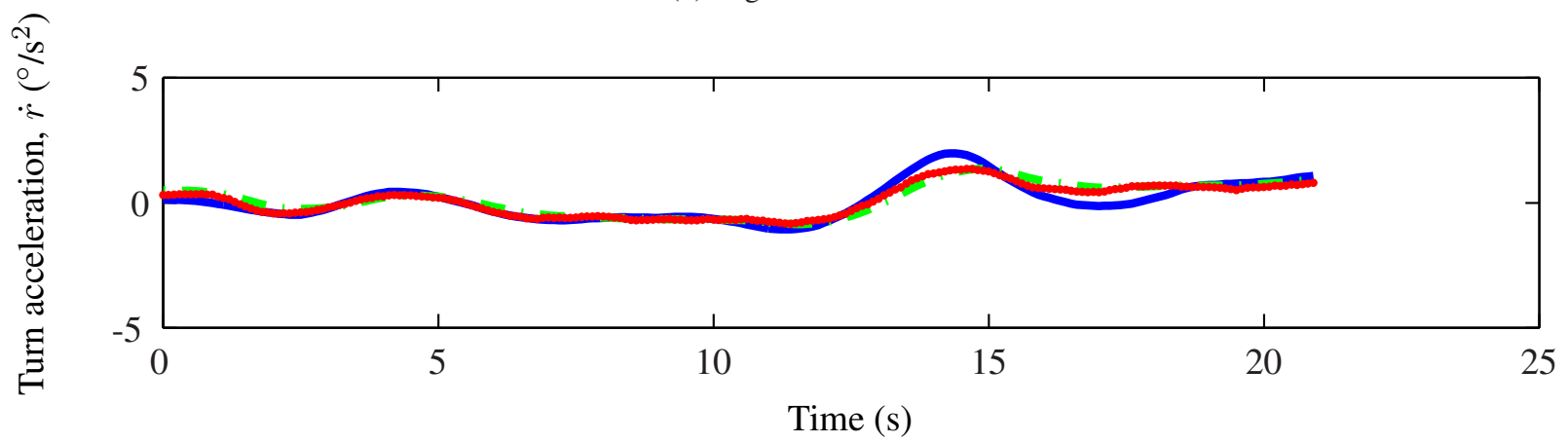

(c) zero turn rate

Figure 5.16: Actual and estimated turn acceleration for the two stable and one unstable turn rate regions 


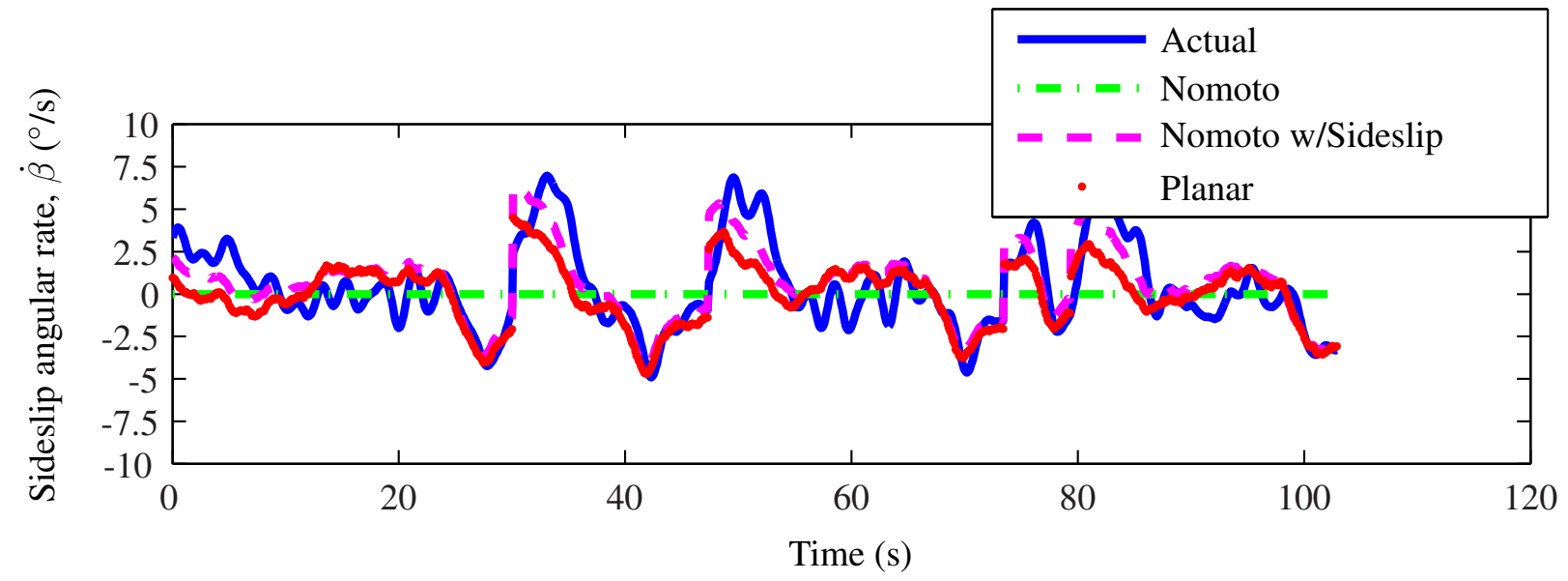

(a) positive turn rate

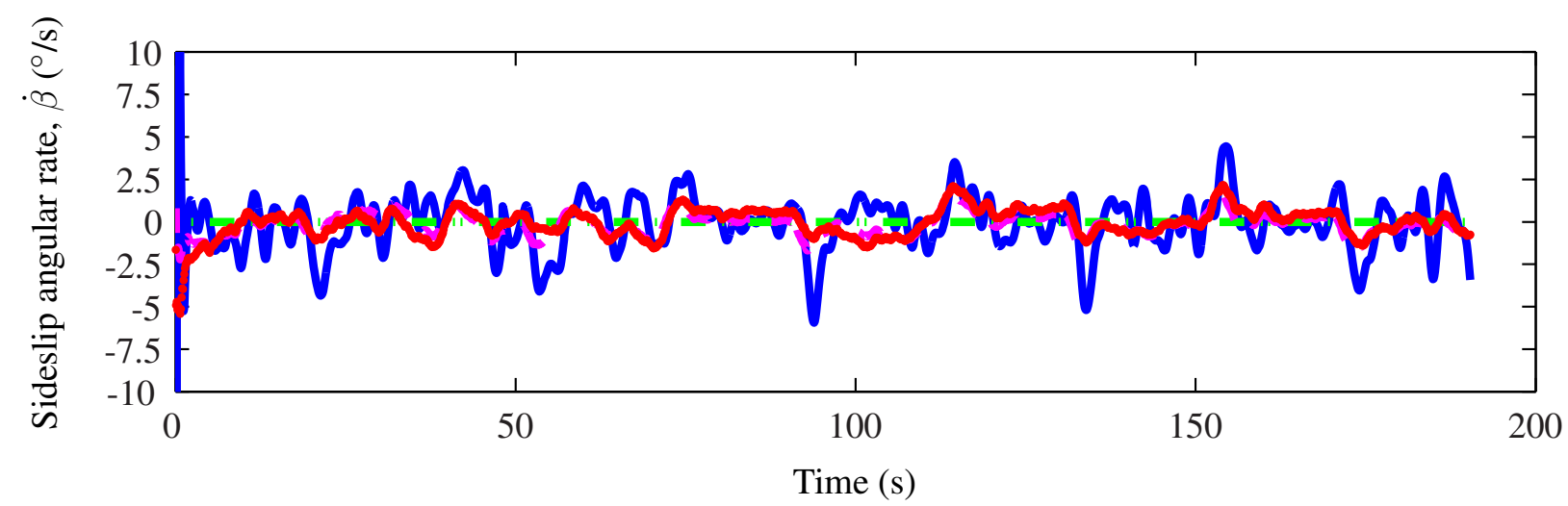

(b) negative turn rate

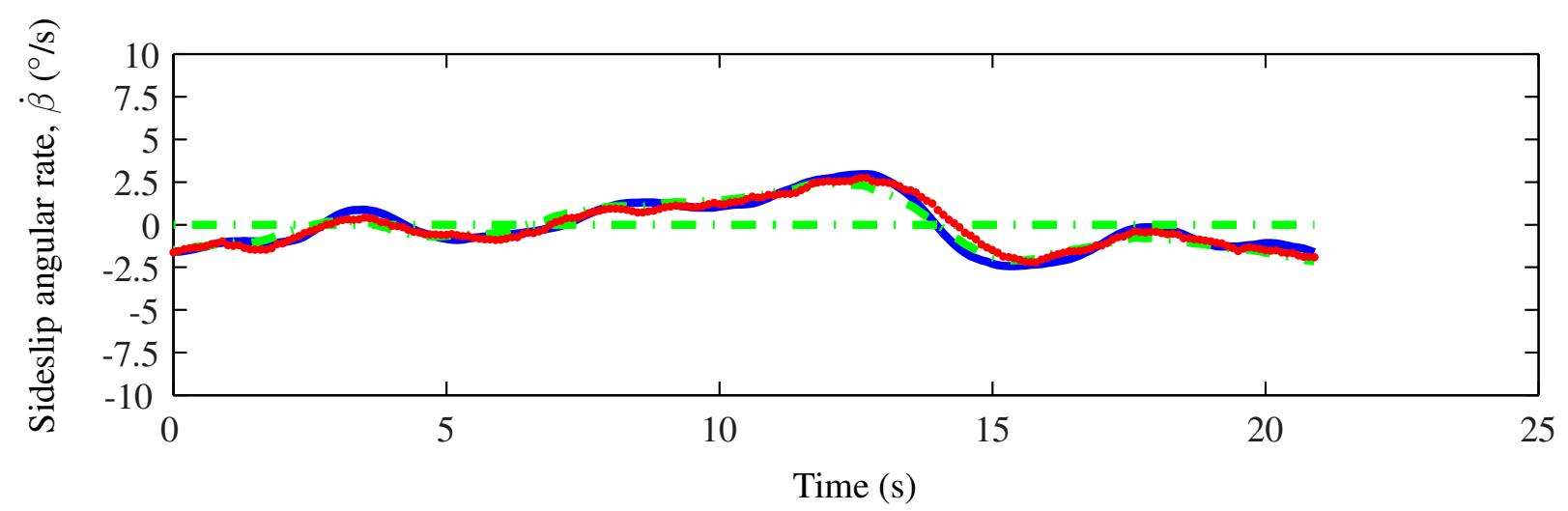

(c) zero turn rate

Figure 5.17: Actual vs. estimated sideslip angular rate for the two stable and one unstable turn rate regions 
measurements, which stabilize the actual Ribcraft USV, are used as open loop inputs to the unstable steering model. The identified model for the unstable system matches the vessel dynamics closely, as confirmed by the validation data. For the data shown in the Figures 5.11 and 5.12, the Nomoto model with sideslip and the linear model best approximate the true course angle history. This observation illustrates the continued need to account for sideslip dynamics in sternward motion. For the stable cases the linear model gives marginally better fits of both sideslip angle and turn rate while the Nomoto model with sideslip best approximates the unstable zero turn rate region.

Table 5.7 shows parameter identification cost function values for identified models of the Ribcraft USV sternward steering dynamics. These values were computed using the the same cost function, (5.18), used for forward motion parameter identification, which computes costs using the validation data sets shown in Figures 5.18 and 5.19. As previously qualitatively noted, the linear steering model best approximates the stable turn rate dynamics, while the simple Nomoto model best accounts for unstable steering dynamics about the zero turn rate equilibrium. However, like in the forward motion case, the added complexity of including two additional parameters for the linear model is considered not worth the slight edge in turn rate estimation.

\begin{tabular}{|c|c|c|c|}
\hline Turn Rate Region & Nomoto & Nomoto w/ Side Slip & Linear \\
\hline positive & 15.7 & 4.4 & 4.0 \\
\hline negative & 11.9 & 4.6 & 3.0 \\
\hline zero & 4.7 & 2.2 & 7.4 \\
\hline
\end{tabular}

Table 5.8 shows the parameter values obtained for the linear steering model (4.21) for sternward motion. Table 5.9 shows the parameter values for the Nomoto model with sideslip (4.24) for sternward motion. Table 5.10 shows the parameter values for the Nomoto model (4.22) for sternward motion. Like for forward motion, $a_{*}$ and $b_{*}$ refer to the elements $*$ in the state matrix $\boldsymbol{A}$ and input matrix $\boldsymbol{B}$ respectively. If $a$ and $b$ 


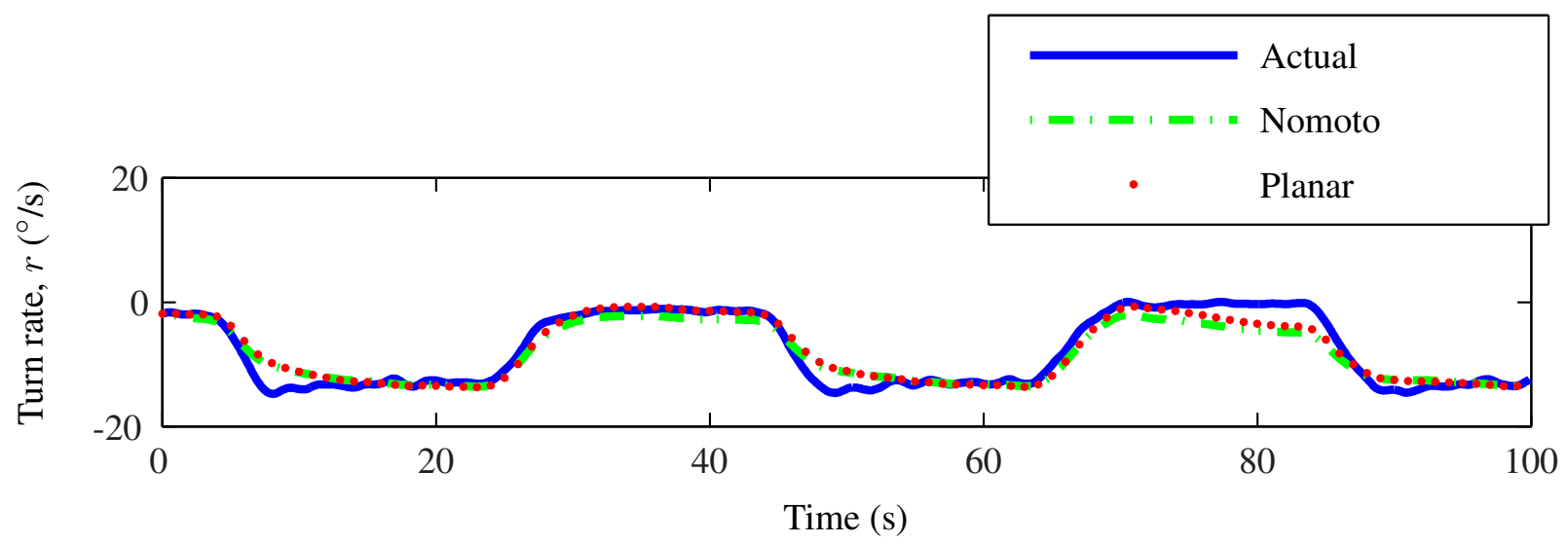

(a) positive turn rate

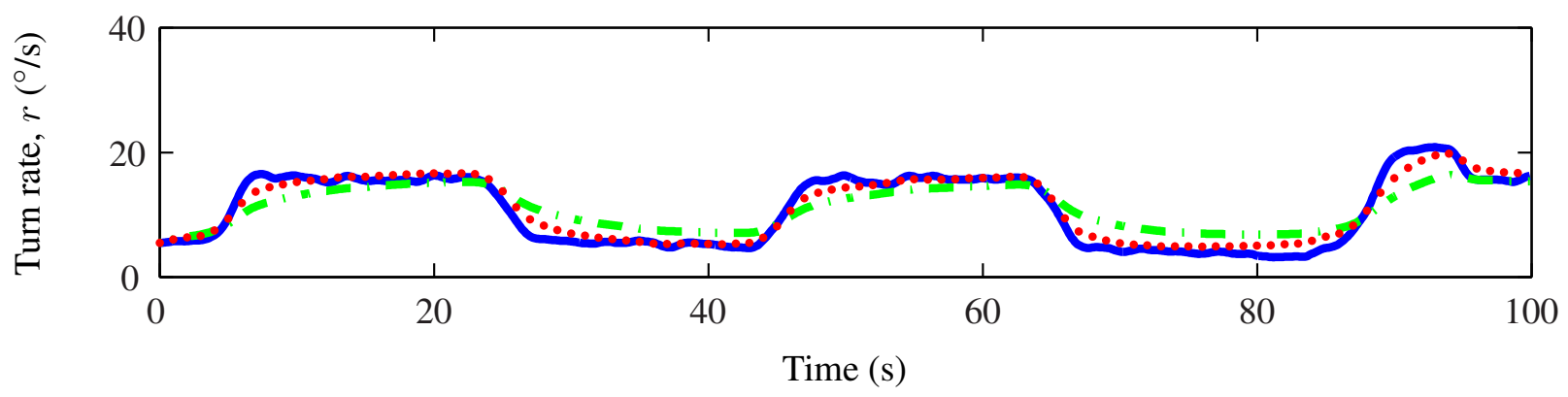

(b) negative turn rate

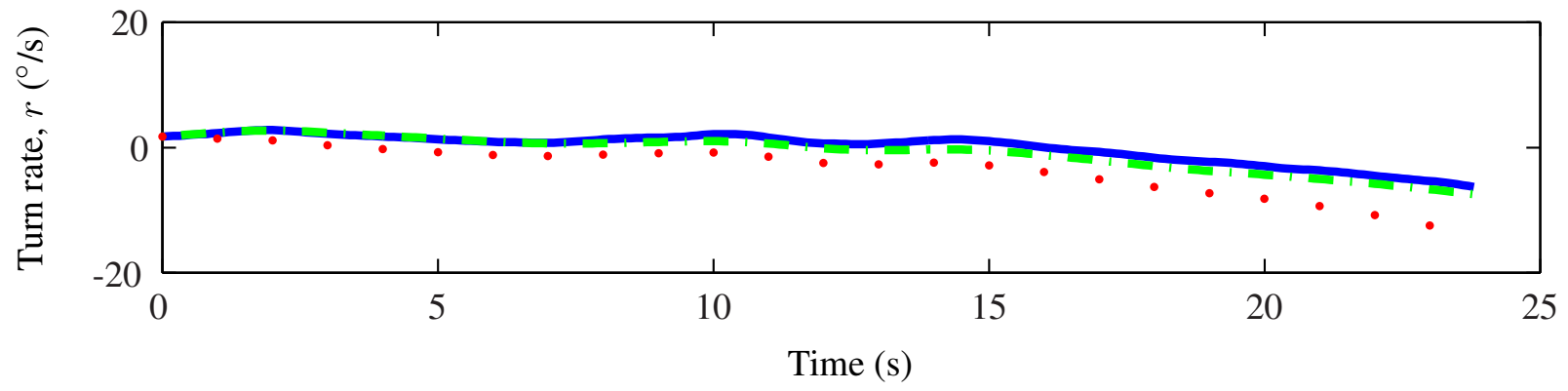

(c) zero turn rate

Figure 5.18: Actual and simulated turn rate time histories for the two stable and one unstable turn rate regions 


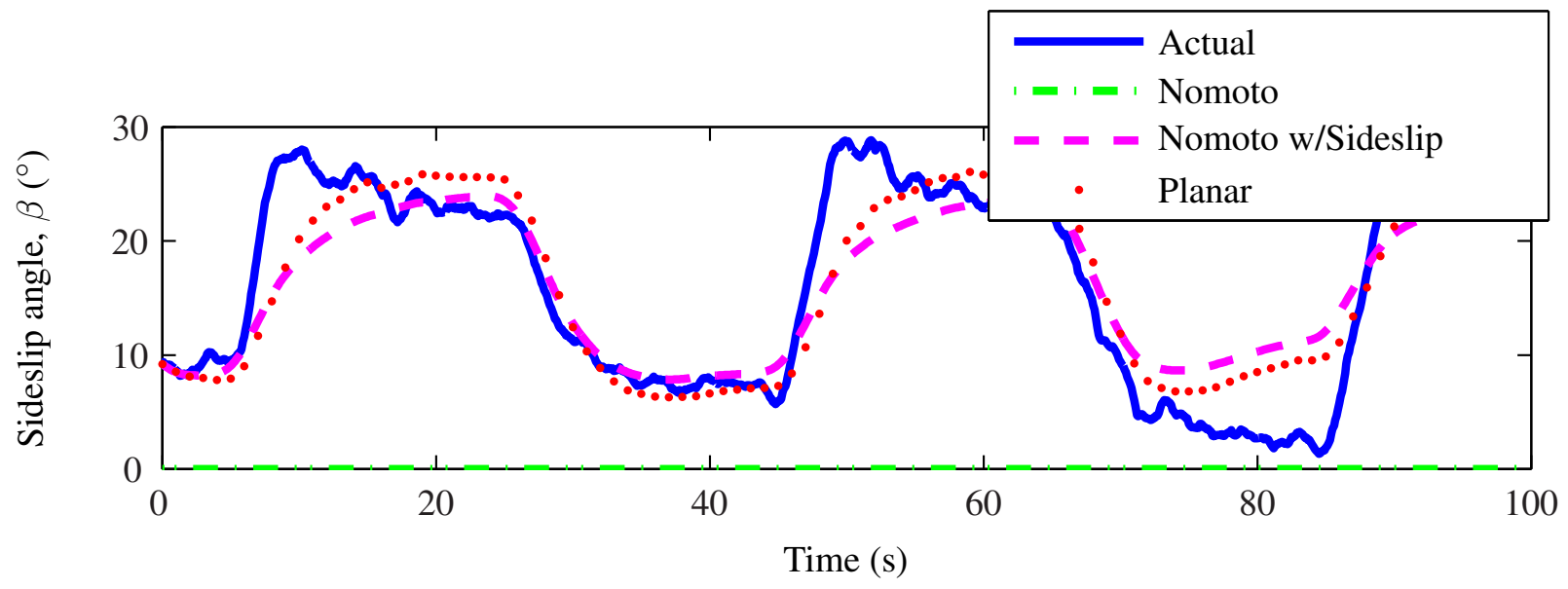

(a) positive turn rate

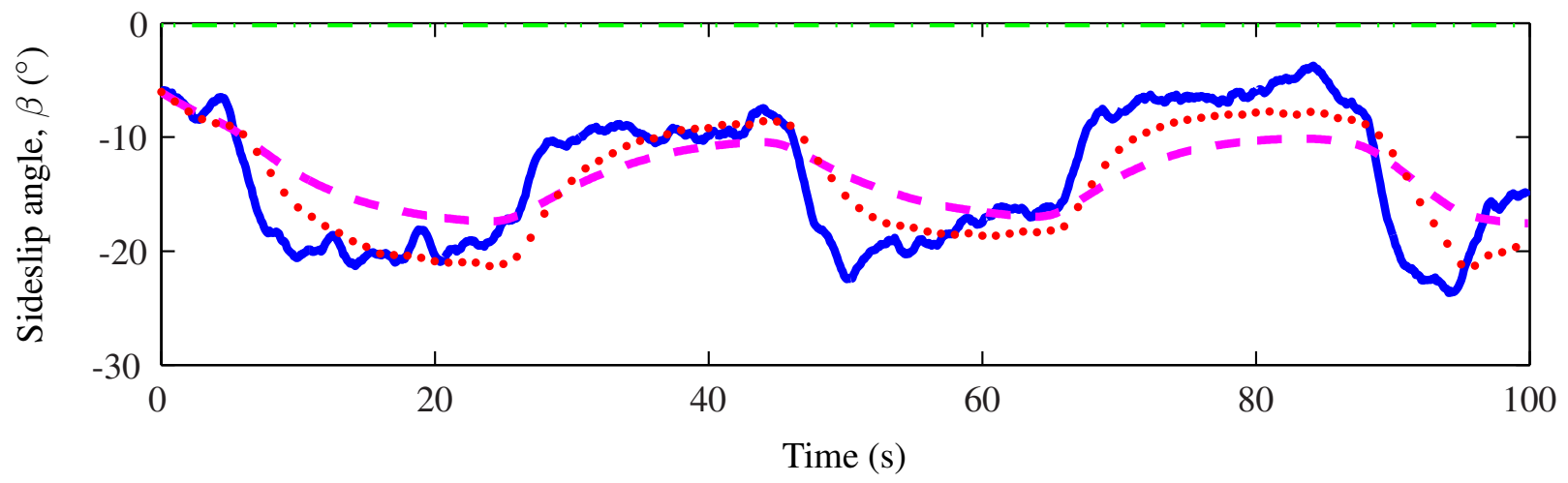

(b) negative turn rate

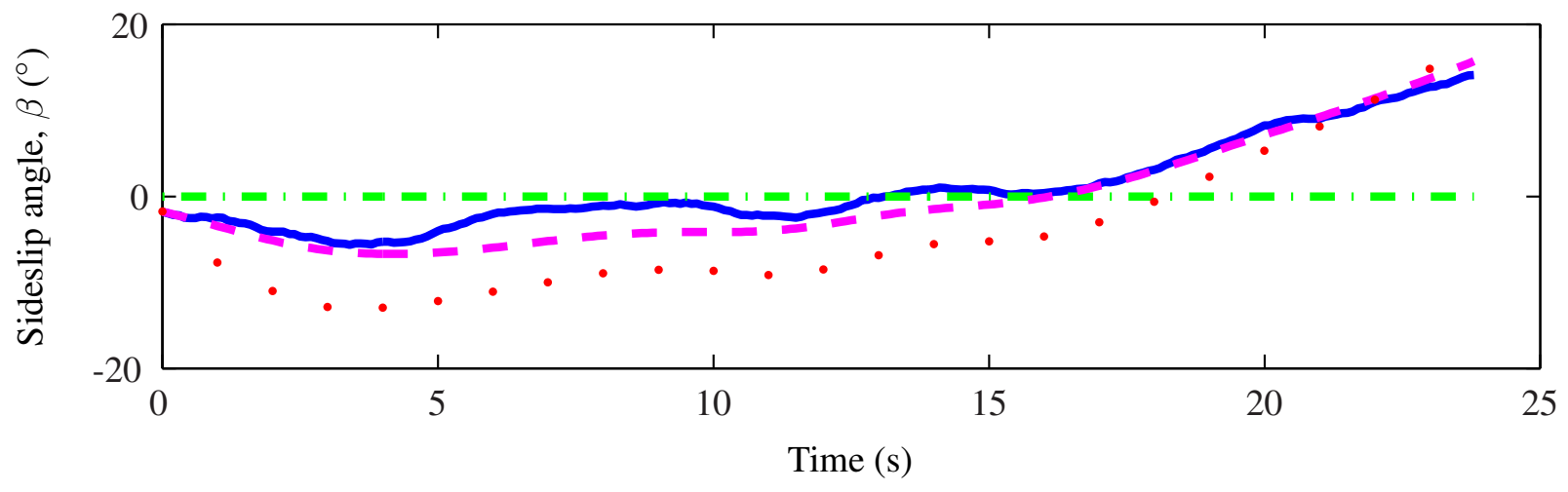

(c) zero turn rate

Figure 5.19: Actual vs. simulated sideslip angle time histories for the two stable and one unstable turn rate regions 
Table 5.8: Linear steering model parameter values for sternward motion

\begin{tabular}{c|cccccccc} 
Turn Rate Region & $a_{11}$ & $a_{12}$ & $a_{21}$ & $a_{22}$ & $b_{1}$ & $b_{1_{\text {bias }}}$ & $b_{2}$ & $b_{2_{\text {bias }}}$ \\
\hline positive & -0.58 & 0.022 & -2.36 & -0.15 & -0.0019 & -0.078 & 0.12 & -0.15 \\
negative & -0.37 & 0.012 & 0.077 & -0.34 & -0.0014 & -0.0029 & 0.14 & 4.47 \\
zero & -0.66 & 0.040 & 7.09 & -0.20 & -0.0045 & -0.078 & 0.18 & 1.64
\end{tabular}

Table 5.9: Nomoto-with-sideslip steering model parameter values for sternward motion

\begin{tabular}{c|cccccc} 
Turn Rate Region & $a_{\beta}$ & $b_{\beta}$ & $b_{\beta_{\text {bias }}}$ & $a_{r}$ & $b_{r}$ & $b_{r_{\text {bias }}}$ \\
\hline positive & -0.51 & -0.74 & 2.74 & -0.27 & 0.13 & 0.15 \\
negative & -0.28 & -0.26 & -1.45 & -0.21 & 0.07 & 2.24 \\
zero & -0.34 & -1.10 & 1.05 & 0.12 & 0.10 & 0.688
\end{tabular}

appear without a subscript, then the state and input matrices are scalars.

Table 5.10: Nomoto steering model parameter values for sternward motion

\begin{tabular}{c|ccc} 
Turn Rate Region & $a_{r}$ & $b_{r}$ & $b_{r_{\text {bias }}}$ \\
\hline positive & -0.27 & 0.13 & 0.15 \\
negative & -0.21 & 0.07 & 2.24 \\
zero & 0.12 & 0.10 & 0.68
\end{tabular}

Examining Tables 5.2 and 5.7, one finds it is reasonable to use the Nomoto-with-sideslip model at speeds below $2.6 \mathrm{~m} / \mathrm{s}$ and to use the simpler Nomoto model at higher speeds. Table 5.11 shows the parameter values corresponding to the steering model adopted for the given reference speed. Combined with the the first order linear speed model (4.18) and the bilinear thrust model (4.10), this multiple-model representation for the steering dynamics enables one to generate dynamically feasible reference trajectories for the Ribcraft USV. Note that though the nonlinear speed model works best in sternward motion, the linear model is implemented to provide continuity between forward and sternward models. Using the linear model potentially results in errors in steady state behavior; however, these errors are not realized when used to control the vehicle. The resulting trajectories may then be tracked using algorithms developed in Chapter 6. 
Table 5.11: Speed and steering model parameter values. (Parameter units suppressed.)

\begin{tabular}{c|c|cc|cccccc}
$\begin{array}{c}\text { Forward } \\
\text { Speed (m/s) }\end{array}$ & $\begin{array}{c}\text { Turn } \\
\text { Region }\end{array}$ & $a_{u}$ & $b_{u_{\text {bias }}}$ & $a_{\beta}$ & $b_{\beta}$ & $b_{\beta_{\text {bias }}}$ & $a_{r}$ & $b_{r}$ & $b_{r_{\text {bias }}}$ \\
\hline-1.0 & positive & -0.19 & 0 & -0.51 & -0.74 & 2.74 & -0.27 & 0.13 & 0.15 \\
-1.0 & negative & -0.19 & 0 & -0.28 & -0.26 & -1.45 & -0.21 & 0.07 & 2.24 \\
-1.0 & zero & -0.19 & 0 & -0.34 & -1.10 & 1.05 & 0.12 & 0.10 & 0.688 \\
1.1 & N/A & -0.12 & 0.07 & -0.140 & -0.20 & 0.061 & -0.34 & -1.06 & 0.29 \\
1.5 & N/A & -0.31 & 0.33 & -0.135 & -0.10 & 0.008 & -0.40 & -1.11 & 0.77 \\
2.6 & N/A & -0.58 & 0.85 & 0.035 & -0.01 & 0.042 & -0.39 & -0.33 & 0.78 \\
3.0 & N/A & -1.09 & 2.19 & N/A & N/A & N/A & -0.52 & -0.30 & 1.10 \\
3.2 & N/A & -1.00 & 1.75 & N/A & N/A & N/A & -0.69 & -0.33 & 1.40 \\
4.0 & N/A & -0.17 & -1.12 & N/A & N/A & N/A & -1.29 & -0.46 & 2.62 \\
7.6 & N/A & -0.06 & -1.65 & N/A & N/A & N/A & -1.08 & -0.39 & 2.47 \\
9.2 & N/A & -0.10 & -1.31 & N/A & N/A & N/A & -0.29 & -0.10 & 0.65
\end{tabular}




\title{
CHAPTER
}

\author{
6
}

\section{TRAJECTORY TRACKING}

Two trajectory tracking controllers are formulated to control the Ribcraft USV, whose dynamics are modeled using the linear speed model and the Nomoto-with-sideslip steering model with speed-scheduled parameters. First, the problem of tracking a reference trajectory is formulated by defining tracking error dynamics. (Recall that the generation of these reference trajectories is not a focus of this dissertation though the process makes use of the models developed in Chapters 4 and 5.) Next, we develop a cascade of proportionalderivative (PD) controllers whose inputs are the reference heading and speed signals, modified based on the tracking error. As an alternative to the PD cascade, we describe a nonlinear tracking control law, derived through backstepping. The "inner loop" of the backstepping control law is based on results described in [Jiang and Nijmeijer, 1997, Jiang et al., 2001]. By including turn rate scheduled steering dynamics, the backstepping control law is extended to consider closed-loop sternward trajectory tracking. An additional augmentation to the backstepping control law yields global asymptotic control in the presence of constant 
or slowly varying flow. Both control laws are implemented on the Ribcraft USV and the experimental results are compared for forward trajectory tracking. The backstepping control law is also implemented for sternward motion on the Ribcraft USV. Simulations confirm the backstepping control law's ability to account for constant or slowly varying flow.

\subsection{Problem Formulation}

A riverine vessel moving in still water obeys the following kinematics:

$$
\begin{aligned}
& \dot{x}=V \sin \chi \\
& \dot{y}=V \cos \chi
\end{aligned}
$$

with $x$ and $y$ representing the position of the boat in cartesian coordinates, $V$ representing the speed of the boat, and $\chi$ representing the course angle. The speed and steering dynamics are estimated using the Nomoto model with sideslip, the linear speed model, and the assumption that $V \approx u$, giving

$$
\begin{gathered}
\dot{u}=a_{u} u+\tilde{T}+b_{u_{\mathrm{bias}}} \\
\left(\begin{array}{c}
\dot{\beta} \\
\dot{\psi} \\
\dot{r}
\end{array}\right)=\left(\begin{array}{ccc}
a_{\beta} & 0 & b_{\beta} \\
0 & 0 & 1 \\
0 & 0 & a_{r}
\end{array}\right)\left(\begin{array}{l}
\beta \\
\psi \\
r
\end{array}\right)+\left(\begin{array}{c}
0 \\
0 \\
b_{r}
\end{array}\right) \tilde{T} \delta s+\left(\begin{array}{c}
b_{\beta_{\text {bias }}} \\
0 \\
b_{r_{\text {bias }}}
\end{array}\right)
\end{gathered}
$$

where $\beta$ is the sideslip angle, $\psi$ is the vessel heading, $r$ is the vessel turn rate, $\delta s$ is the rudder input, and $\tilde{T}$ is the thrust produced by the propeller, divided by the unknown parameter $\left(m-X_{\dot{u}}\right)$. Recall from (5.7) that

$$
\tilde{T}=\tilde{c} u \delta n+\tilde{d} \delta n|\delta n|
$$

The parameters $a_{u}, b_{u_{\text {bias }}}, a_{\beta}, b_{\beta}, b_{\beta_{\text {bias }}}, a_{r}, b_{r}$, and $b_{r_{\text {bias }}}$ are scheduled by nominal equilibrium speed $u_{0}$. Note that the course angle $\chi$ satisfies

$$
\chi=\psi+\beta .
$$


To simplify controller development, we recast the dynamics (6.4) in terms of $\psi, r$, and $\chi$, giving

$$
\left(\begin{array}{c}
\dot{\chi} \\
\dot{\psi} \\
\dot{r}
\end{array}\right)=\left(\begin{array}{ccc}
a_{\beta} & -a_{\beta} & 1+b_{\beta} \\
0 & 0 & 1 \\
0 & 0 & a_{r}
\end{array}\right)\left(\begin{array}{c}
\chi \\
\psi \\
r
\end{array}\right)+\left(\begin{array}{c}
0 \\
0 \\
b_{r}
\end{array}\right) \tilde{T} \delta s+\left(\begin{array}{c}
b_{\beta_{\mathrm{bias}}} \\
0 \\
b_{r_{\mathrm{bias}}}
\end{array}\right)
$$

with eigenvalues of $a_{\beta}, 0$, and $a_{r}$.

Assume a reference trajectory has been generated based upon an initial state and reference rudder and propeller speed inputs. Specifically,

$$
\begin{aligned}
\dot{x}_{\mathrm{r}} & =u_{\mathrm{r}} \sin \chi_{\mathrm{r}} \\
\dot{y}_{\mathrm{r}} & =u_{r} \cos \chi_{\mathrm{r}} \\
\left(\begin{array}{c}
\dot{\chi}_{\mathrm{r}} \\
\dot{\psi}_{\mathrm{r}} \\
\dot{r}_{\mathrm{r}}
\end{array}\right) & =\left(\begin{array}{ccc}
a_{\beta} & -a_{\beta} & 1+b_{\beta} \\
0 & 0 & 1 \\
0 & 0 & a_{r}
\end{array}\right)\left(\begin{array}{c}
\chi_{\mathrm{r}} \\
\psi_{\mathrm{r}} \\
r_{\mathrm{r}}
\end{array}\right)+\left(\begin{array}{c}
0 \\
0 \\
b_{r}
\end{array}\right) \tilde{T}_{r} \delta s_{\mathrm{r}}+\left(\begin{array}{c}
b_{\beta_{\text {bias }}} \\
0 \\
b_{r_{\text {bias }}}
\end{array}\right) \\
\dot{u}_{\mathrm{r}} & =a_{u} u_{\mathrm{r}}+\tilde{T}_{\mathrm{r}}+b_{u_{\text {bias }}}
\end{aligned}
$$

(Since we have assumed that $V \approx u$, in normal operation, we also require that $V_{\mathrm{r}} \approx u_{\mathrm{r}}$.) The trajectory tracking error is

$$
\begin{aligned}
\left(\begin{array}{c}
e_{s} \\
e_{y}
\end{array}\right) & =\boldsymbol{R}(\chi)\left(\begin{array}{c}
x_{\mathrm{r}}-x \\
y_{\mathrm{r}}-y
\end{array}\right) \\
e_{u} & =u_{\mathrm{r}}-u \\
e_{\chi} & =\chi_{\mathrm{r}}-\chi \\
e_{\psi} & =\psi_{\mathrm{r}}-\psi \\
\dot{e}_{\psi} & =r_{\mathrm{r}}-r
\end{aligned}
$$


Differentiating the error variables gives

$$
\begin{aligned}
\dot{e}_{s} & =\left(\dot{\chi}_{\mathrm{r}}-a_{\beta} e_{\chi}+a_{\beta} e_{\psi}-\left(1+b_{\beta}\right) \dot{e}_{\psi}\right) e_{y}-u_{\mathrm{r}}+e_{u}+u_{\mathrm{r}} \cos e_{\chi} \\
\dot{e}_{y} & =-\left(\dot{\chi}_{\mathrm{r}}-a_{\beta} e_{\chi}+a_{\beta} e_{\psi}-\left(1+b_{\beta}\right) \dot{e}_{\psi}\right) e_{s}+u_{\mathrm{r}} \sin e_{\chi} \\
\left(\begin{array}{c}
\dot{e}_{\chi} \\
\dot{e}_{\psi} \\
\ddot{e}_{\psi}
\end{array}\right) & =\left(\begin{array}{ccc}
a_{\beta} & -a_{\beta} & 1+b_{\beta} \\
0 & 0 & 1 \\
0 & 0 & a_{r}
\end{array}\right)\left(\begin{array}{c}
e_{\chi} \\
e_{\psi} \\
\dot{e}_{\psi}
\end{array}\right)+\left(\begin{array}{c}
0 \\
0 \\
b_{r}
\end{array}\right)\left(\tilde{T}_{\mathrm{r}} \delta s_{\mathrm{r}}-\tilde{T} \delta s\right) \\
\dot{e}_{u} & =a_{u} e_{u}+\tilde{T}_{\mathrm{r}}-\tilde{T}
\end{aligned}
$$

Figure 6.1 shows all of the state components, as well as the reference state, and the along track and cross track errors.

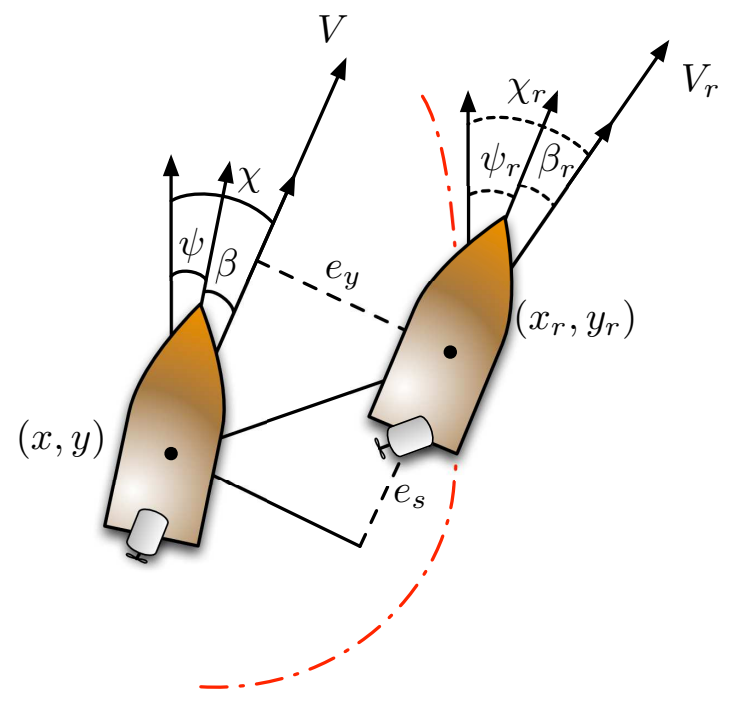

Figure 6.1: Notation and formulation of cross track and along track error. 


\subsection{A Cascaded PD Control Approach}

A simple closed-loop controller incorporating a series of proportional and PD controllers is devised. The cross track and along track error terms are used to adjust the reference speed and heading such that the vessel converges to the correct reference position. Define the speed and heading corrections

$$
\begin{aligned}
& u_{\text {corr }}=k_{p_{s}} e_{s} \\
& \psi_{\text {corr }}=k_{p_{y}} e_{y}
\end{aligned}
$$

To establish a speed and heading that drive the vessel toward the desired trajectory, we define the following propeller speed and rudder commands

$$
\begin{aligned}
& \delta n=\delta n_{\mathrm{r}}+k_{p_{u}}\left(u_{\mathrm{corr}}+e_{u}\right)+k_{d_{u}}\left(\dot{u}_{\mathrm{corr}}+\dot{e}_{u}\right) \\
& \delta s=\delta s_{\mathrm{r}}+k_{p_{\psi}}\left(\psi_{\mathrm{corr}}+e_{\psi}\right)+k_{d_{\psi}}\left(\dot{\psi}_{\mathrm{corr}}+\dot{e}_{\psi}\right)
\end{aligned}
$$

The control structure can be seen in Figure 6.2.

The closed-loop error dynamics are

$$
\begin{aligned}
\dot{e}_{s} & =\left(\dot{\chi}_{\mathrm{r}}-a_{\beta} e_{\chi}+a_{\beta} e_{\psi}-\left(1+b_{\beta}\right) \dot{e}_{\psi}\right) e_{y}-u_{\mathrm{r}}+e_{u}+u_{\mathrm{r}} \cos e_{\chi} \\
\dot{e}_{y} & =-\left(\dot{\chi}_{\mathrm{r}}-a_{\beta} e_{\chi}+a_{\beta} e_{\psi}-\left(1+b_{\beta}\right) \dot{e}_{\psi}\right) e_{s}+u_{\mathrm{r}} \sin e_{\chi} \\
\left(\begin{array}{c}
\dot{e}_{\chi} \\
\dot{e}_{\psi} \\
\ddot{e}_{\psi}
\end{array}\right) & =\left(\begin{array}{ccc}
a_{\beta} & -a_{\beta} & 1+b_{\beta} \\
0 & 0 & 1 \\
0 & 0 & a_{r}
\end{array}\right)\left(\begin{array}{c}
e_{\chi} \\
e_{\psi} \\
\dot{e}_{\psi}
\end{array}\right)+\left(\begin{array}{c}
0 \\
0 \\
b_{r}
\end{array}\right) f_{\psi}\left(e_{s}, e_{y}, e_{\chi}, e_{\psi}, e_{\dot{\psi}}, e_{u}\right) \\
\dot{e}_{u} & =f_{u}\left(e_{s}, e_{y}, e_{\chi}, e_{\psi}, e_{\dot{\psi}}, e_{u}\right)
\end{aligned}
$$




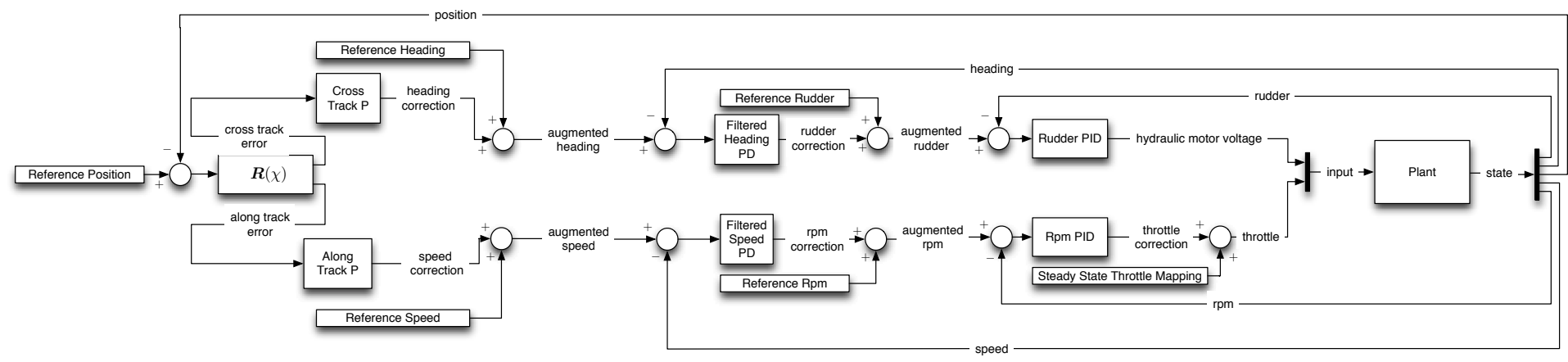

Figure 6.2: Cascaded trajectory tracking controller 


\subsection{A Cascaded PD Control Approach}

where

$$
\begin{aligned}
& f_{\psi}\left(e_{s}, e_{y}, e_{\chi}, e_{\psi}, e_{\dot{\psi}}, e_{u}\right)=\delta s_{r}\left(\tilde{c} \delta n_{r} u_{r}+\tilde{d} \delta n_{r}^{2}\right)-\left(k _ { d _ { \psi } } \left(k_{p_{y}}\left(k_{e_{s}}\left(a_{\beta} e_{\chi}-a_{\beta} e_{\psi}+\left(b_{\beta}+1\right) e_{r}-\dot{\chi}_{r}\right)+u_{r} \sin \left(e_{\chi}\right)\right)\right.\right. \\
& \left.\left.+e_{r}\right)+\delta s_{r}+p_{\psi}\left(e_{\psi}+e_{y} k_{p_{y}}\right)\right)\left(\frac { 1 } { 2 \tilde { d } k _ { d _ { u } } ^ { 2 } } \tilde { c } ( u _ { r } - e _ { u } ) \left(2 a_{\beta} \tilde{d} k_{d_{u}}^{2} e_{\chi} e_{y} k_{p_{s}}-2 a_{\beta} \tilde{d} k_{d_{u}}^{2} e_{\psi} e_{y} k_{p_{s}}\right.\right. \\
& +\left(-4 a_{\beta} \tilde{d} k_{d_{u}}^{2} e_{\chi} e_{y} k_{p_{s}}+4 a_{\beta} \tilde{d} k_{d_{v}}^{2} e_{\psi} e_{y} k_{p_{s}}+4 a_{u} \tilde{d} k_{d_{v}}^{2} e_{u}-4 b_{\beta} \tilde{d} k_{d_{u}}^{2} e_{r} e_{y} k_{p_{s}}+\tilde{c}^{2} k_{d_{u}}^{2} e_{u}^{2}\right. \\
& -2 \tilde{c}^{2} k_{d_{u}}^{2} e_{u} u_{r}+\tilde{c}^{2} k_{d_{u}}^{2} u_{r}^{2}+4 \tilde{c} \tilde{d} \delta n_{r} k_{d_{u}}^{2} u_{r}-2 \tilde{c} k_{d_{u}} e_{u}+2 \tilde{c} k_{d_{v}} u_{r}+4 \tilde{d}^{2} \delta n_{r}^{2} k_{d_{u}}^{2} \\
& +4 \tilde{d} \dot{\chi}_{r} k_{d_{u}}^{2} e_{y} k_{p_{s}}-4 \tilde{d} k_{d_{u}}^{2} e_{r} e_{y} k_{p_{s}}+4 \tilde{d} k_{d_{u}}^{2} e_{u} k_{p_{s}}+4 \tilde{d}_{d_{u}}^{2} k_{p_{s}} u_{r} \cos \left(e_{\chi}\right)-4 \tilde{d} k_{d_{v}}^{2} k_{p_{s}} u_{r} \\
& \left.+4 \tilde{d} \delta n_{r} k_{d_{u}}+4 \tilde{d} k_{d_{u}} e_{s} k_{p_{s}} k_{p_{u}}+4 \tilde{d} k_{d_{u}} e_{u} k_{p_{u}}+1\right)^{\frac{1}{2}}+2 b_{\beta} \tilde{d} k_{d_{u}}^{2} e_{r} e_{y} k_{p_{s}}+\tilde{c} k_{d_{u}} e_{u}-\tilde{c} k_{d_{u}} u_{r} \\
& -2 \tilde{d} \dot{\chi}_{r} k_{d_{u}}^{2} e_{y} k_{p_{s}}+2 \tilde{d} k_{d_{u}}^{2} e_{r} e_{y} k_{p_{s}}-2 \tilde{d} k_{d_{u}}^{2} e_{u} k_{p_{s}}-2 \tilde{d} k_{d_{u}}^{2} k_{p_{s}} u_{r} \cos \left(e_{\chi}\right)+2 \tilde{d} k_{d_{u}}^{2} k_{p_{s}} u_{r} \\
& \left.-2 \tilde{d} \delta n_{r} k_{d_{u}}-2 \tilde{d} k_{d_{u}} e_{s} k_{p_{s}} k_{p_{u}}-2 \tilde{d} k_{d_{u}} e_{u} k_{p_{u}}-1\right)+\frac{1}{4 \tilde{d} k_{d_{u}}^{4}}\left(2 a_{\beta} \tilde{d} k_{d_{u}}^{2} e_{\chi} e_{y} k_{p_{s}}\right. \\
& -2 a_{\beta} \tilde{d} k_{d_{u}}^{2} e_{\psi} e_{y} k_{p_{s}}+\left(-4 a_{\beta} \tilde{d} k_{d_{u}}^{2} e_{\chi} e_{y} k_{p_{s}}+4 a_{\beta} \tilde{d} k_{d_{u}}^{2} e_{\psi} e_{y} k_{p_{s}}+4 a_{u} \tilde{d} k_{d_{u}}^{2} e_{u}\right. \\
& -4 b_{\beta} \tilde{d}_{d_{u}}^{2} e_{r} e_{y} k_{p_{s}}+\tilde{c}^{2} k_{d_{u}}^{2} e_{u}^{2}-2 \tilde{c}^{2} k_{d_{u}}^{2} e_{u} u_{r}+\tilde{c}^{2} k_{d_{u}}^{2} u_{r}^{2}+4 \tilde{c} \tilde{d} \delta n_{r} k_{d_{u}}^{2} u_{r}-2 \tilde{c} k_{d_{u}} e_{u} \\
& +2 \tilde{c} k_{d_{u}} u_{r}+4 \tilde{d}^{2} \delta n_{r}^{2} k_{d_{u}}^{2}+4 \tilde{d} \dot{\chi}_{r} k_{d_{u}}^{2} e_{y} k_{p_{s}}-4 \tilde{d}_{d_{u}}^{2} e_{r} e_{y} k_{p_{s}}+4 \tilde{d} k_{d_{u}}^{2} e_{u} k_{p_{s}} \\
& \left.+4 \tilde{d} k_{d_{u}}^{2} k_{p_{s}} u_{r} \cos \left(e_{\chi}\right)-4 \tilde{d}_{d_{u}}^{2} k_{p_{s}} u_{r}+4 \tilde{d} \delta n_{r} k_{d_{u}}+4 \tilde{d} k_{d_{u}} e_{s} k_{p_{s}} k_{p_{u}}+4 \tilde{d} k_{d_{u}} e_{u} k_{p_{u}}+1\right)^{\frac{1}{2}} \\
& +2 b_{\beta} \tilde{d} k_{d_{u}}^{2} e_{r} e_{y} k_{p_{s}}+\tilde{c} k_{d_{u}} e_{u}-\tilde{c} k_{d_{u}} u_{r}-2 \tilde{d} \dot{\chi}_{r} k_{d_{u}}^{2} e_{y} k_{p_{s}}+2 \tilde{d} k_{d_{u}}^{2} e_{r} e_{y} k_{p_{s}}-2 \tilde{d} k_{d_{u}}^{2} e_{u} k_{p_{s}} \\
& \left.\left.-2 \tilde{d} k_{d_{u}}^{2} k_{p_{s}} u_{r} \cos \left(e_{\chi}\right)+2 \tilde{d} k_{d_{u}}^{2} k_{p_{s}} u_{r}-2 \tilde{d} \delta n_{r} k_{d_{u}}-2 \tilde{d} k_{d_{u}} e_{s} k_{p_{s}} k_{p_{u}}-2 \tilde{d} k_{d_{u}} e_{u} k_{p_{u}}-1\right)^{2}\right)
\end{aligned}
$$


and

$$
\begin{aligned}
f_{u}\left(e_{s}, e_{y}, e_{\chi}, e_{\psi}, e_{\dot{\psi}}, e_{u}\right) & =\frac{1}{2 \tilde{d} k_{d_{u}}^{2}}\left(2 a_{\beta} \tilde{d} k_{d_{u}}^{2} e_{\chi} e_{y} k_{p_{s}}-2 a_{\beta} \tilde{d} k_{d_{u}}^{2} e_{\psi} e_{y} k_{p_{s}}+\left(-4 a_{\beta} \tilde{d} k_{d_{u}}^{2} e_{\chi} e_{y} k_{p_{s}}\right.\right. \\
& +4 a_{\beta} \tilde{d} k_{d_{u}}^{2} e_{\psi} e_{y} k_{p_{s}}+4 a_{u} \tilde{d} k_{d_{u}}^{2} e_{u}-4 b_{\beta} \tilde{d} k_{d_{u}}^{2} e_{r} e_{y} k_{p_{s}}+\tilde{c}^{2} k_{d_{u}}^{2}\left(e_{u}-u_{r}\right)^{2} \\
& +2 \tilde{c} k_{d_{u}}\left(2 \tilde{d} \delta n_{r} k_{d_{u}} u_{r}-e_{u}+u_{r}\right)+4 \tilde{d}^{2} \delta n_{r}^{2} k_{d_{u}}^{2}+4 \tilde{d} \dot{\chi}_{r} k_{d_{u}}^{2} e_{y} k_{p_{s}}-4 \tilde{d} k_{d_{u}}^{2} e_{r} e_{y} k_{p_{s}} \\
& +4 \tilde{d} k_{d_{u}}^{2} e_{u} k_{p_{s}}+4 \tilde{d} d_{u}^{2} k_{p_{s}} u_{r} \cos \left(e_{\chi}\right)-4 \tilde{d} k_{d_{u}}^{2} k_{p_{s}} u_{r}+4 \tilde{d} \delta n_{r} k_{d_{u}}+4 \tilde{d} k_{d_{u}} e_{s} k_{p_{s}} k_{p_{u}} \\
& \left.+4 \tilde{d} k_{d_{u}} e_{u} k_{p_{u}}+1\right)^{\frac{1}{2}}+2 b_{\beta} \tilde{d} k_{d_{u}}^{2} e_{r} e_{y} k_{p_{s}}+\tilde{c} k_{d_{u}} e_{u}-\tilde{c} k_{d_{u}} u_{r}-2 \tilde{d} \dot{\chi}_{r} k_{d_{u}}^{2} e_{y} k_{p_{s}} \\
& +2 \tilde{d} k_{d_{u}}^{2} e_{r} e_{y} k_{p_{s}}-2 \tilde{d} k_{d_{u}}^{2} e_{u} k_{p_{s}}-2 \tilde{d} k_{d_{u}}^{2} k_{p_{s}} u_{r} \cos \left(e_{\chi}\right)+2 \tilde{d} k_{d_{u}}^{2} k_{p_{s}} u_{r}-2 \tilde{d} \delta n_{r} k_{d_{u}} \\
& \left.-2 \tilde{d} k_{d_{u}} e_{s} k_{p_{s}} k_{p_{u}}-2 \tilde{d} k_{d_{u}} e_{u} k_{p_{u}}-1\right)
\end{aligned}
$$

The error dynamics are nonlinear and time-varying. However, linearizing about a reference trajectory with constant reference speed and course rate results in a linear, time-invariant system.

Linearizing the error dynamics (6.24-6.27) gives

$$
\begin{gathered}
\left(\begin{array}{c}
\dot{e}_{s} \\
\dot{e}_{y} \\
\dot{e}_{\chi} \\
\dot{e}_{\psi} \\
\ddot{e}_{\psi} \\
\dot{e}_{u}
\end{array}\right)=\left(\begin{array}{ccc}
0 & \dot{\chi}_{\mathrm{r}} & 0 \\
-\dot{\chi}_{\mathrm{r}} & 0 & u_{\mathrm{r}} \\
0 & 0 & -a_{\beta} \\
0 & 0 & a_{\beta} \\
a_{51} & a_{52} & -b_{r} k_{d_{\psi}} k_{p_{y}} u_{r}\left(\tilde{c} u_{r} \varphi_{3}+\varphi_{3}^{2}\right) \\
-\frac{k_{p_{s}} k_{p_{u}}}{k_{d_{u}}}\left(\frac{1}{\varphi_{1}}+1\right) & -\dot{\chi}_{r} k_{p_{s}}\left(\frac{1}{\varphi_{1}}+1\right) & 0 \\
0 & 0 & 0 \\
0 & 0 & 0 \\
b_{\beta}+1 & 0 & 0 \\
0 & 1 & a_{56} \\
-b_{r} k_{p_{\psi}}\left(\tilde{c} u_{r} \varphi_{3}+\varphi_{3}^{2}\right) & a_{r}-b_{r} k_{d_{\psi}}\left(\tilde{c} u_{r} \varphi_{3}+\varphi_{3}^{2}\right) \\
0 & 0 & a_{66}
\end{array}\right)\left(\begin{array}{c}
e_{s} \\
e_{y} \\
e_{\chi} \\
e_{\psi} \\
\dot{e}_{\psi} \\
e_{u}
\end{array}\right)
\end{gathered}
$$


where

$$
\begin{aligned}
\varphi_{1} & =\sqrt{\tilde{c}^{2} k_{d_{u}}^{2} u_{r}^{2}+4 \tilde{c} \tilde{d} \delta n_{r} k_{d_{u}}^{2} u_{r}+2 \tilde{c} k_{d_{u}} u_{r}+4 \tilde{d}^{2} \delta n_{r}^{2} k_{d_{u}}^{2}+4 \tilde{d} \delta n_{r} k_{d_{u}}+1} \\
\varphi_{2} & =\frac{\sqrt{\tilde{c}^{2} k_{d_{u}}^{2} u_{r}^{2}+4 \tilde{c} \tilde{d} \delta n_{r} k_{d_{u}}^{2} u_{r}+2 \tilde{c} k_{d_{u}} u_{r}+4 \tilde{d}^{2} \delta n_{r}^{2} k_{d_{u}}^{2}+4 \tilde{d} \delta n_{r} k_{d_{u}}+1}-\tilde{c} k_{d_{u}} u_{r}-2 \tilde{d} \delta n_{r} k_{d_{u}}-1}{2 \tilde{d} k_{d_{u}}^{2}} \\
\varphi_{3} & =\frac{-\sqrt{\tilde{c}^{2} k_{d_{u}}^{2} u_{r}^{2}+4 \tilde{c} \tilde{d} \delta n_{r} k_{d_{u}}^{2} u_{r}+2 \tilde{c} k_{d_{u}} u_{r}+4 \tilde{d}^{2} \delta n_{r}^{2} k_{d_{u}}^{2}+4 \tilde{d} \delta n_{r} k_{d_{u}}+1}-\tilde{c} k_{d_{u}} u_{r}-2 \tilde{d} \delta n_{r} k_{d_{u}}-1}{2 \tilde{d} k_{d_{u}}^{2}} \\
\varphi_{4} & =4 a_{u} \tilde{d} k_{d_{u}}^{2}-2 \tilde{c}^{2} k_{d_{u}}^{2} u_{r}-2 \tilde{c} k_{d_{u}}+4 \tilde{d} k_{d_{u}}^{2} k_{p_{s}}+4 \tilde{d} k_{d_{u}} k_{p_{u}}
\end{aligned}
$$

and

$$
\begin{aligned}
& a_{51}=b_{r}\left(\dot{\chi}_{r} k_{d_{\psi}} k_{p_{y}}\left(\tilde{c} u_{r} \varphi_{2}+\varphi_{2}^{2}\right)-\frac{\delta s_{r}}{k_{d_{v}}}\left(\tilde{c} u_{r} k_{p_{s}} k_{p_{u}}\left(\frac{1}{\varphi_{1}}-1\right)+\varphi_{2}\left(\frac{2 \tilde{d} k_{p_{s}} k_{p_{u}}}{\varphi_{1}}-2 \tilde{d} k_{p_{s}} k_{p_{u}}\right)\right)\right) \\
& a_{52}=b_{r}\left(-k_{p_{\psi}} k_{p_{y}}\left(\tilde{c} u_{r} \varphi_{3}+\varphi_{3}^{2}\right)+\delta s_{r}\left(\tilde{c} u_{r} \dot{\chi}_{r} k_{p_{s}}\left(\frac{1}{\varphi_{1}}+1\right)+\varphi_{3}\left(\frac{2 \tilde{d} \dot{\chi}_{r} k_{p_{s}}}{\varphi_{1}}+2 \tilde{d} \dot{\chi}_{r} k_{p_{s}}\right)\right)\right) \\
& a_{56}=-b_{r} \delta s_{r}\left(\left(-\frac{\varphi_{4}}{2 \varphi_{1}}+\tilde{c} k_{d_{u}}-2 \tilde{d} k_{d_{u}}^{2} k_{p_{s}}-2 \tilde{d} k_{d_{u}} k_{p_{u}}\right)\left(\frac{\tilde{c} u_{r}}{2 \tilde{d} k_{d_{u}}^{2}}+1\right)+\frac{\varphi_{3}}{k_{d_{u}}^{2}}-\tilde{c} \varphi_{3}\right) \\
& a_{66}=\frac{1}{2 \tilde{d} k_{d_{u}}^{2}}\left(-\frac{\varphi_{4}}{2 \varphi_{1}}+\tilde{c} k_{d_{u}}-2 \tilde{d} k_{d_{u}}^{2} k_{p_{s}}-2 \tilde{d} k_{d_{u}} k_{p_{u}}\right)
\end{aligned}
$$

Assume that $u_{\mathrm{r}}, \delta n_{\mathrm{r}}$, and $\dot{\chi}_{\mathrm{r}}$ are constants, which makes (6.30) an LTI system.

We analyze closed-loop stability of the PD cascaded control system by finding eigenvalues of (6.30), which is populated using identified models shown in Table 5.5 and control gains obtained through experimental tuning shown in Table 6.1. Table 6.2 shows the system eigenvalues for a range of operating conditions. All eigenvalues are in the left half plane, indicating exponential stability of the LTI system (6.30). Note that the eigenvalues are dependent upon $u_{\mathrm{r}}$ and $\dot{\chi}_{\mathrm{r}}$. The nominal value $\dot{\chi}_{\mathrm{r}}=0$ is used. Only five eigenvalues exist for higher speeds since the Nomoto steering model is used. 
Table 6.1: Control Gains for the PD control system for the Ribcraft USV.

\begin{tabular}{c|cccccc}
$u_{\mathrm{r}}(\mathrm{m} / \mathrm{s})$ & $k_{p_{y}}$ & $k_{p_{\psi}}$ & $k_{d_{\psi}}$ & $k_{p_{s}}$ & $k_{p_{V}}$ & $k_{d_{V}}$ \\
\hline 1.1 & 0.4 & -1.0 & -0.5 & 0.1 & 50 & 50 \\
1.5 & 0.4 & -1.0 & -0.75 & 0.1 & 50 & 50 \\
2.6 & 0.4 & -0.6 & -1.0 & 0.1 & 50 & 50 \\
3.0 & 0.4 & -0.8 & -0.9 & 0.1 & 50 & 50 \\
3.2 & 0.4 & -0.8 & -0.9 & 0.1 & 50 & 50 \\
4.0 & 0.4 & -1.0 & -0.5 & 0.1 & 50 & 50 \\
7.6 & 0.3 & -0.5 & -0.5 & 0.1 & 50 & 50 \\
9.2 & 0.2 & -0.4 & -1.0 & 0.1 & 50 & 50
\end{tabular}

Table 6.2: Ribcraft USV Closed-Loop Linearized PD Control System Eigenvalues

\begin{tabular}{c|cccccc}
$u_{\mathrm{r}}(\mathrm{m} / \mathrm{s})$ & $\lambda_{1}$ & $\lambda_{2}$ & $\lambda_{3}$ & $\lambda_{4}$ & $\lambda_{5}$ & $\lambda_{6}$ \\
\hline 1.1 & -5.47 & -0.31 & $-0.01+0.31 \imath$ & $-0.01-0.31 \imath$ & -0.19 & -0.04 \\
1.5 & -5.21 & -0.43 & $-0.02+0.39 \imath$ & $-0.02-0.39 \imath$ & -0.16 & -0.04 \\
2.6 & -5.02 & -0.44 & $-0.01+0.27 \imath$ & $-0.01-0.27 \imath$ & -0.04 & \\
3.0 & -4.51 & -0.55 & $-0.01+0.28 \imath$ & $-0.01-0.28 \imath$ & -0.04 & \\
3.2 & -4.60 & -0.70 & $-0.02+0.27 \imath$ & $-0.02-0.27 \imath$ & -0.04 & \\
4.0 & -5.43 & -1.16 & $-0.01+0.31 \imath$ & $-0.01+0.31 \imath$ & -0.04 & \\
7.6 & -5.57 & -1.07 & $-0.03+0.36 \imath$ & $-0.03-0.36 \imath$ & -0.04 & \\
9.2 & -5.54 & -0.34 & $-0.03+0.46 \imath$ & $-0.03-0.46 \imath$ & -0.04 &
\end{tabular}

\subsection{A Backstepping Control Approach}

Alternatively, one may use backstepping to attenuate the error terms $e_{s}, e_{y}, e_{u}, e_{\chi}$, and $e_{\psi}$. Take the adjusted position error to be

$$
\left(\begin{array}{c}
e_{s} \\
e_{y}
\end{array}\right)=\kappa_{5} \boldsymbol{R}(\chi)\left(\begin{array}{c}
x_{\mathrm{r}}-x \\
y_{\mathrm{r}}-y
\end{array}\right)
$$

where $\kappa_{5}$ is a constant parameter. (This parameter will ultimately play the role of a turn rate gain in the inner loop of the backstepping controller.) The rates of change of the along track and cross track error are

$$
\left(\begin{array}{c}
\dot{e}_{s} \\
\dot{e}_{y}
\end{array}\right)=\left(\begin{array}{c}
\dot{\chi} e_{y}+\kappa_{5}\left(-u_{\mathrm{r}}+e_{u}+u_{\mathrm{r}} \cos e_{\chi}\right) \\
-\dot{\chi} e_{s}+\kappa_{5} u_{\mathrm{r}} \sin e_{\chi}
\end{array}\right)
$$


We transform and group the tracking error variables as follows:

$$
\left(\begin{array}{c}
\boldsymbol{\eta} \\
\hdashline \boldsymbol{\xi} \\
\hdashline e_{\psi}
\end{array}\right)=\left(\begin{array}{c}
\eta_{1} \\
\eta_{2} \\
\eta_{3} \\
\hdashline \xi_{1} \\
\xi_{2} \\
\hdashline e_{\psi}
\end{array}\right)=\left(\begin{array}{c}
e_{s} \\
e_{y} \\
e_{\chi} \\
\hdashline-a_{\beta} e_{\psi}+\left(b_{\beta}+1\right) \dot{e}_{\psi} \\
e_{u} \\
e_{\psi}
\end{array}\right)
$$

The error dynamics, in the transformed variables, are

$$
\begin{aligned}
\dot{\eta}_{1} & =\left(\dot{\chi}_{\mathrm{r}}-a_{\beta} \eta_{3}-\xi_{1}\right) \eta_{2}+\kappa_{5}\left(-u_{\mathrm{r}}+\xi_{2}+u_{\mathrm{r}} \cos \eta_{3}\right) \\
\dot{\eta}_{2} & =-\left(\dot{\chi}_{\mathrm{r}}-a_{\beta} \eta_{3}-\xi_{1}\right) \eta_{1}+\kappa_{5} u_{\mathrm{r}} \sin \eta_{3} \\
\dot{\eta}_{3} & =a_{\beta} \eta_{3}+\xi_{1} \\
\dot{\xi}_{1} & =\frac{a_{r}\left(b_{\beta}+1\right)-a_{\beta}}{1+b_{\beta}} \xi_{1}+\frac{\left(a_{r}\left(b_{\beta}+1\right)-a_{\beta}\right) a_{\beta}}{1+b_{\beta}} e_{\psi}+\left(b_{\beta} b_{r}+b_{r}\right)\left(\tilde{T}_{\mathrm{r}} \delta s_{\mathrm{r}}-\tilde{T} \delta s\right) \\
\dot{\xi}_{2} & =a_{u} \xi_{2}+\tilde{T}_{\mathrm{r}}-\tilde{T} \\
\left(\begin{array}{c}
\dot{e}_{\psi} \\
\ddot{e}_{\psi}
\end{array}\right) & =\left(\begin{array}{cc}
0 & 1 \\
0 & a_{r}
\end{array}\right)\left(\begin{array}{c}
e_{\psi} \\
\dot{e}_{\psi}
\end{array}\right)+\left(\begin{array}{c}
0 \\
b_{r}
\end{array}\right)\left(\tilde{T}_{\mathrm{r}} \delta s_{\mathrm{r}}-\tilde{T} \delta s\right)
\end{aligned}
$$

Following in the spirit of [Jiang et al., 2001,Jiang and Nijmeijer, 1997] we define the following virtual inputs $\xi_{1}=\phi_{1}(\eta)$ and $\xi_{2}=\phi_{2}(\eta)$ to stabilize the $\dot{\boldsymbol{\eta}}$ subsystem (6.34), (6.35), and (6.36):

$$
\begin{aligned}
& \xi_{1}=\phi_{1}(\boldsymbol{\eta})=-\frac{\sin \eta_{3}}{\eta_{3}} \eta_{2} u_{\mathrm{r}} \kappa_{5}-\kappa_{1} \tanh \left(\kappa_{2} \eta_{3}\right)-a_{\beta} \eta_{3} \\
& \xi_{2}=\phi_{2}(\boldsymbol{\eta})=u_{\mathrm{r}}-u_{\mathrm{r}} \cos \eta_{3}-\kappa_{3} \tanh \left(\kappa_{4} \eta_{1}\right)
\end{aligned}
$$

where the parameters $\kappa_{1}, \kappa_{2}, \kappa_{3}, \kappa_{4}$, and $\kappa_{5}$ are positive tuning factors that adjust controller sensitivity and amplitude. The candidate Lyapunov function

$$
V_{1}(\boldsymbol{\eta})=\frac{1}{2} \eta_{1}^{2}+\frac{1}{2} \eta_{2}^{2}+\frac{1}{2} \eta_{3}^{2}
$$


is positive definite. The derivative of the candidate Lyapunov function is

$$
\begin{aligned}
\dot{V}_{1}= & \eta_{1} \dot{\eta}_{1}+\eta_{2} \dot{\eta}_{2}+\eta_{3} \dot{\eta}_{3} \\
= & \eta_{1}\left(\left(\dot{\chi}_{\mathrm{r}}-a_{\beta} \eta_{3}-\xi_{1}\right) \eta_{2}-\kappa_{5}\left(u_{\mathrm{r}}-\xi_{2}-u_{\mathrm{r}} \cos \eta_{3}\right)\right)+\eta_{2}\left(-\left(\dot{\chi}_{\mathrm{r}}-a_{\beta} \eta_{3}-\xi_{1}\right) \eta_{1}+\kappa_{5} u_{\mathrm{r}} \sin \eta_{3}\right) \\
& +\eta_{3}\left(a_{\beta} \eta_{3}+\xi_{1}\right) \\
= & -\kappa_{3} \kappa_{5} \eta_{1} \tanh \left(\kappa_{4} \eta_{1}\right)+\eta_{1} \eta_{2}\left(\dot{\chi}_{\mathrm{r}}-a_{\beta} \eta_{3}-\xi_{1}\right)-\eta_{1} \eta_{2}\left(\dot{\chi}_{\mathrm{r}}-a_{\beta} \eta_{3}-\xi_{1}\right)+\kappa_{5} \eta_{2} u_{\mathrm{r}} \sin \eta_{3} \\
& +\eta_{3}\left(-\frac{\sin \eta_{3}}{\eta_{3}} \kappa_{5} \eta_{2} u_{\mathrm{r}}-\kappa_{1} \tanh \left(\kappa_{2} \eta_{3}\right)\right) \\
= & -\kappa_{3} \kappa_{5} \eta_{1} \tanh \left(\kappa_{4} \eta_{1}\right)-\kappa_{1} \eta_{3} \tanh \left(\kappa_{2} \eta_{3}\right) \leq 0
\end{aligned}
$$

Lemma 6.3.1. Assume that $\chi_{\mathrm{r}}$ and $u_{\mathrm{r}}$ are bounded. Also, assume that $u_{\mathrm{r}}(t) \neq 0$ on $[0, \infty)$. All trajectories of (6.34), (6.35), and (6.36) with inputs defined by (6.40) and (6.41) are globally uniformly bounded and

$$
\lim _{t \rightarrow \infty}|\boldsymbol{\eta}|=0
$$

Proof: Since the candidate Lyapunov function is positive definite and radially unbounded and the candidate Lyapunov function's derivative is negative semidefinite, $\boldsymbol{\eta}$ is uniformly bounded and defined for $t \geq 0$. Note that the Lyapunov function's second derivative is bounded. By Barbalat's lemma, $\eta_{1}$ and $\eta_{3}$ converge to zero. To prove that $\eta_{2}$ converges to zero note that $\eta_{3} \rightarrow 0$ as $t \rightarrow \infty$ and

$$
\frac{\sin \theta}{\theta}=\int_{0}^{1} \cos (s \theta) d s
$$

so $\int_{0}^{1} \cos \left(s \eta_{3}\right) d s \rightarrow 1$ as $t \rightarrow \infty$. Recall that $u_{\mathrm{r}}(t) \neq 0$ for all $t \geq 0$. The $\eta_{3}$ dynamics of the closed-loop system are

$$
\dot{\eta}_{3}=-\kappa_{1} \tanh \left(\kappa_{2} \eta_{3}\right)+\kappa_{5} \eta_{2} u_{\mathrm{r}} \int_{0}^{1} \cos \left(s \eta_{3}\right) d s
$$

Note that $\eta_{3}$ tends to 0 so $\eta_{2} u_{\mathrm{r}} \int_{0}^{1} \cos \left(s \eta_{3}\right) d s$ must go to 0 , which implies that $\eta_{2} \rightarrow 0$.

Lemma 6.3.2. The equilibrium state $\boldsymbol{\eta}=\mathbf{0}$ for the system (6.34-6.36) under the feedback control law (6.40-6.41) is exponentially stable for continuous and bounded $u_{\mathrm{r}} \neq 0$ and $\dot{\chi}_{\mathrm{r}}$. 
Proof: The linearized closed-loop system around the equilibrium point is

$$
\dot{\boldsymbol{\eta}}=\left(\begin{array}{ccc}
-\kappa_{3} \kappa_{4} \kappa_{5} & \dot{\chi}_{\mathrm{r}} & 0 \\
-\dot{\chi}_{\mathrm{r}} & 0 & \kappa_{5} u_{\mathrm{r}} \\
0 & -\kappa_{5} u_{\mathrm{r}} & -\kappa_{1} \kappa_{2}
\end{array}\right) \boldsymbol{\eta}
$$

The system (6.43) is exponentially stable if

$$
\boldsymbol{P} \boldsymbol{A}(t)+\boldsymbol{A}^{T}(t) \boldsymbol{P}=-\boldsymbol{Q}(t)
$$

where $\boldsymbol{P}$ and $\boldsymbol{Q}(t)$ are positive definite symmetric matrices. Define a constant, positive definite matrix

$$
\boldsymbol{P}=\left(\begin{array}{ccc}
p_{1} & 0 & 0 \\
0 & p_{2} & p_{3} \\
0 & p_{3} & p_{4}
\end{array}\right)
$$

From (6.44), we have

$$
\boldsymbol{Q}(t)=\left(\begin{array}{ccc}
2 p_{1} \kappa_{3} \kappa_{4} \kappa_{5} & -\dot{\chi}_{\mathrm{r}}\left(p_{1}-p_{2}\right) & \dot{\chi}_{\mathrm{r}} p_{3} \\
-\dot{\chi}_{\mathrm{r}}\left(p_{1}-p_{2}\right) & 2 p_{3} u_{\mathrm{r}} \kappa_{5} & \left(p_{4}-p_{2}\right) u_{\mathrm{r}} \kappa_{5}-p_{3} \kappa_{1} \kappa_{2} \\
\dot{\chi}_{\mathrm{r}} p_{3} & \left(p_{4}-p_{2}\right) u_{\mathrm{r}} \kappa_{5}-p_{3} \kappa_{1} \kappa_{2} & 2 p_{4} \kappa_{1} \kappa_{2}-2 u_{\mathrm{r}} \kappa_{5} p_{3}
\end{array}\right)
$$

To simplify analysis, set $p_{1}=p_{2}$ and $p_{3}=\frac{p_{1}-p_{4}}{\operatorname{sgn}\left(u_{\mathrm{r}}\right)}$. Then

$$
\boldsymbol{Q}(t)=\left(\begin{array}{ccc}
2 p_{1} \kappa_{3} \kappa_{4} \kappa_{5} & 0 & \frac{\dot{\chi}_{\mathrm{r}}(t)\left(p_{1}-p_{4}\right)}{\operatorname{sgn}\left(u_{\mathrm{r}}\right)} \\
0 & 2\left(p_{1}-p_{4}\right)\left|u_{\mathrm{r}}(t)\right| \kappa_{5} & 0 \\
\frac{\dot{\mathrm{x}}_{\mathrm{r}}(t)\left(p_{1}-p_{4}\right)}{\operatorname{sgn}\left(u_{\mathrm{r}}\right)} & 0 & 2 p_{4} \kappa_{1} \kappa_{2}-2\left|u_{\mathrm{r}}(t)\right| \kappa_{5}\left(p_{1}-p_{4}\right)
\end{array}\right)
$$

The matrix $\boldsymbol{Q}(t)$ is positive definite provided the leading principal minors are positive. Setting $p_{4}>0$ and 
$p_{1}=p_{4}+\epsilon$ where $\epsilon>0$ is arbitrarily small, the leading principal minors are

$$
\begin{aligned}
2 p_{1} \kappa_{3} \kappa_{4} \kappa_{5} & >0 \\
4 p_{1} p_{3}\left|u_{\mathrm{r}}\right| \kappa_{3} \kappa_{4} \kappa_{5}^{2}>0 & >\dot{\chi}_{\mathrm{r}}^{2} \kappa_{5}\left(p_{1}-p_{4}\right)^{3}\left|u_{\mathrm{r}}\right|+8 \kappa_{1} \kappa_{2} \kappa_{3} \kappa_{4} \kappa_{5}^{2} p_{1}\left(p_{1}-p_{4}\right) p_{4}\left|u_{\mathrm{r}}\right|-8 \kappa_{3} \kappa_{4} \kappa_{5}^{3} p_{1}\left(p_{1}-p_{4}\right)^{2} u_{\mathrm{r}}^{2}>0
\end{aligned}
$$

Thus, by Lyapunov's indirect method, the time-varying closed-loop system (6.34-6.36), with feedback control law (6.40-6.41), is locally exponentially stable.

Setting the inputs to

$$
\begin{aligned}
\left(\begin{array}{c}
\tilde{T} \delta s \\
\tilde{T}
\end{array}\right)= & \left(\begin{array}{c}
\tilde{T}_{\mathrm{r}} \delta s_{\mathrm{r}} \\
\tilde{T}_{\mathrm{r}}
\end{array}\right)+\left(\begin{array}{cc}
\frac{1}{b_{r}\left(b_{\beta}+1\right)} & 0 \\
0 & 1
\end{array}\right)\left(\left(\begin{array}{c}
\frac{a_{r}\left(b_{\beta}+1\right)-a_{\beta}}{1+b_{\beta}} \xi_{1}+\frac{\left(a_{r}\left(b_{\beta}+1\right)-a_{\beta}\right) a_{\beta}}{1+b_{\beta}} e_{\psi} \\
a_{u} \xi_{2}
\end{array}\right)\right. \\
& -\left(\begin{array}{c}
\left(-\left(\int_{0}^{1} \cos \left(s \eta_{3}\right) d s\right) \kappa_{5} u_{\mathrm{r}}\right)\left(-\left(\dot{\chi}_{\mathrm{r}}-a_{\beta} \eta_{3}+\xi_{1}\right) \eta_{1}+\kappa_{5} u_{\mathrm{r}} \sin \eta_{3}\right) \\
-\kappa_{3} \kappa_{4} \operatorname{sech}^{2}\left(\kappa_{4} \eta_{1}\right)\left(\left(\dot{\chi}_{\mathrm{r}}-a_{\beta} \eta_{3}+\xi_{1}\right) \eta_{2}+\kappa_{5}\left(-u_{\mathrm{r}}+u_{\mathrm{r}} \cos \eta_{3}+\xi_{2}\right)\right)
\end{array}\right) \\
& -\left(\begin{array}{c}
\left(-\left(\int_{0}^{1} \sin \left(s \eta_{3}\right) s d s\right) \eta_{2} \kappa_{5} u_{\mathrm{r}}-\kappa_{1} \kappa_{2} \operatorname{sech}^{2}\left(\kappa_{2} \eta_{3}\right)-a_{\beta}\right)\left(a_{\beta} \eta_{3}+\xi_{1}\right) \\
u_{\mathrm{r}} \sin \eta_{3}\left(a_{\beta} \eta_{3}+\xi_{1}\right)
\end{array}\right) \\
& \left.+\left(\begin{array}{c}
\eta_{3} \\
\eta_{1}
\end{array}\right)+\boldsymbol{K}\left(\begin{array}{c}
\xi_{1}+\left(\int_{0}^{1} \cos \left(s \eta_{3}\right) d s\right) \eta_{2} \kappa_{5} u_{\mathrm{r}}+\kappa_{1} \tanh \left(\kappa_{2} \eta_{3}\right)+a_{\beta} \eta_{3} \\
\xi_{2}-u_{\mathrm{r}}+u_{\mathrm{r}} \cos \eta_{3}+\kappa_{3} \tanh \left(\kappa_{4} \eta_{1}\right)
\end{array}\right)\right)
\end{aligned}
$$

asymptotically stabilizes the equilibrium $\boldsymbol{\eta}=\mathbf{0}$ and $\boldsymbol{\xi}=\mathbf{0}$ if $\beta_{\mathrm{r}}, \chi_{\mathrm{r}}, \delta s_{\mathrm{r}}, u_{\mathrm{r}}, \delta n_{\mathrm{r}}$ are bounded, the matrix

$$
\boldsymbol{K}=\left(\begin{array}{ll}
k_{1} & k_{2} \\
k_{2} & k_{3}
\end{array}\right)
$$

is positive definite, and $\tilde{T} \neq 0$. The rudder angle may be found directly from (5.6) and the propeller speed is

$$
\delta n=\frac{-\tilde{c} u+\sqrt{(\tilde{c} u)^{2}+4 \tilde{d} \tilde{T}}}{2 \tilde{d}}
$$

Figure 6.3 shows the dynamics of the transformed tracking error variables (6.33) separated into constituent 
subsystems where $\boldsymbol{K}_{\mathrm{ctrl}}$ is the control law, (6.46) combined with the thrust mapping (6.47) to output thrust angle, $\delta s$, and propeller speed, $\delta n$.

Proposition 6.3.3. The control law (6.46) globally asymptotically stabilizes the dynamic system (6.34-6.38) about the equilibrium $\boldsymbol{\eta}=\mathbf{0}$ and $\boldsymbol{\xi}=\mathbf{0}$ and $e_{\psi}$ remains bounded and converges asymptotically to zero provided $\beta_{\mathrm{r}}, \chi_{\mathrm{r}}, \delta s_{\mathrm{r}}, u_{\mathrm{r}}$, and $\delta n_{\mathrm{r}}$ are bounded, $\tilde{T} \neq 0$, and $-\frac{a_{\beta}}{1+b_{\beta}}<0$.

Proof: The proof of Proposition 6.3.3 follows from Lyapunov stability analysis of the closed-loop system [Khalil, 2002]. For simplicity, we consider only the special case in which the parameters $a_{\beta}$ and $b_{\beta}$ do not change values due to model switching; the discontinuities that are introduced by these isolated changes in parameter values can be addressed with slight modifications of the Lyapunov function [Liberzon, 2003]. Consider the error dynamics in (6.34-6.38). Choose the radially unbounded candidate Lyapunov function

$$
V_{2}=\frac{1}{2} \eta_{1}^{2}+\frac{1}{2} \eta_{2}^{2}+\frac{1}{2} \eta_{3}^{2}+\frac{1}{2}(\boldsymbol{\xi}-\boldsymbol{\phi}(\boldsymbol{\eta}))^{T}(\boldsymbol{\xi}-\boldsymbol{\phi}(\boldsymbol{\eta}))>0
$$

Applying the control law (6.46), the time derivative of (6.48) is

$$
\dot{V}_{2}=-\kappa_{3} \eta_{1} \tanh \left(\kappa_{4} \eta_{1}\right)-\kappa_{1} \eta_{3} \tanh \left(\kappa_{1} \eta_{3}\right)-(\boldsymbol{\xi}-\boldsymbol{\phi}(\boldsymbol{\eta}))^{T} \boldsymbol{K}(\boldsymbol{\xi}-\boldsymbol{\phi}(\boldsymbol{\eta})) \leq 0
$$

Using Lemma 6.3.1 for the $\boldsymbol{\eta}$ subsystem, and recalling that $\boldsymbol{K}$ is positive definite, the equilibrium is globally asymptotically stable by Lyapunov's direct method, provided $e_{\psi}$ is bounded so that the input (6.46) remains well-defined.

Rearranging the $\xi_{1}$ transform in (6.33) gives

$$
\dot{e}_{\psi}=\frac{a_{\beta}}{b_{\beta}+1} e_{\psi}+\frac{1}{b_{\beta}+1} \xi_{1}
$$

Since $\frac{a_{\beta}}{1+b_{\beta}}<0$, the system (6.49) is exponentially stable. Since $\xi_{1}$ remains bounded while the input (6.46) is well-defined, $e_{\psi}\left(\right.$ and $\dot{e}_{\psi}$ ) remains bounded; see Lemma 4.6 in [Khalil, 2002]. Furthermore, as $\xi_{1}$ converges to zero, so do $e_{\psi}$ and $\dot{e}_{\psi}$.

At higher speeds, the sideslip dynamics become extremely stable and $\beta$ remains very close to zero. Rather 


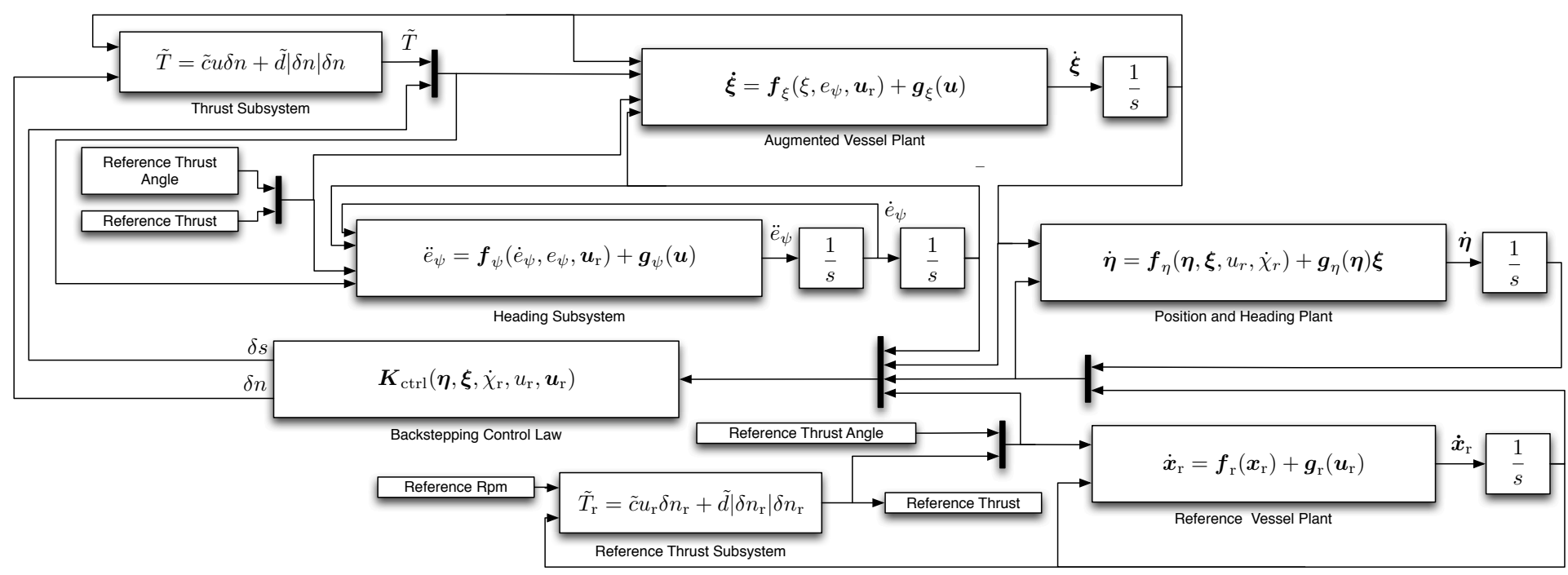

Figure 6.3: Backstepping feedback system 
than vary the structure of the speed-scheduled steering dynamics by eliminating $\beta$ as a state variable, we set $a_{\beta}=0$ and $b_{\beta}=0$ with the understanding that $\beta$ remains zero in this higher speed regime. Referring to (6.33), we see that $\xi_{1}=\dot{e}_{\psi}$, in this case. The dynamics (6.39) are then superfluous; the $\xi_{1}$ dynamics are given by (6.37). In this scenario the control law (6.46) globally asymptotically stabilizes (6.34-6.38).

In an effort to understand similarities between the PD control law and the backstepping control law we linearize the error dynamics (6.16-6.19) controlled by (6.46) giving

$$
\left(\begin{array}{c}
\dot{e}_{s} \\
\dot{e}_{y} \\
\dot{e}_{\chi} \\
\dot{e}_{\psi} \\
\ddot{e}_{\psi} \\
\dot{e}_{u}
\end{array}\right)=\left(\begin{array}{ccc}
0 & \dot{\chi}_{\mathrm{r}} & 0 \\
-\dot{\chi}_{\mathrm{r}} & 0 & u_{\mathrm{r}} \kappa_{5} \\
0 & 0 & a_{\beta} \\
0 & 0 & 0 \\
\frac{\left(a_{\beta}+\dot{\chi}_{\mathrm{r}}\right) u_{\mathrm{r}} \kappa_{5}-k_{2} \kappa_{3} \kappa_{4}}{b_{\beta}+1} & -\frac{k_{1} u_{\mathrm{r}} \kappa 5}{b_{\beta}+1} & a_{53} \\
-k_{3} \kappa_{3} \kappa_{4}-1 & -\dot{\chi}_{\mathrm{r}} \kappa_{3} \kappa_{4}-k_{2} u_{\mathrm{r}} \kappa_{5} & -k_{2}\left(a_{\beta}+\kappa_{1} \kappa_{2}\right) \\
0 & 0 & \kappa_{5} \\
0 & 0 & 0 \\
a_{\beta} & b_{\beta}+1 & 0 \\
\frac{a_{\beta}\left(a_{\beta}+k_{1}+\kappa_{1} \kappa_{2}\right)}{b_{\beta}+1} & -\frac{a_{\beta} b_{\beta}+\left(b_{\beta}+1\right)\left(k_{1}+\kappa_{1} \kappa_{2}\right)}{b_{\beta}+1} & -\frac{k_{2}}{b_{\beta}+1} \\
a_{\beta} k_{2} & \left(-b_{\beta}-1\right) k_{2} & -k_{3}-\kappa_{3} \kappa_{4}
\end{array}\right)\left(\begin{array}{c}
e_{s} \\
e_{y} \\
e_{\chi} \\
e_{\psi} \\
\dot{e}_{\psi} \\
e_{u}
\end{array}\right)
$$

where

$$
a_{53}=-\frac{a_{\beta}^{2}+k_{1} a_{\beta}+\kappa_{1} \kappa_{2} a_{\beta}+u_{r}^{2} \kappa_{5}^{2}+k_{1} \kappa_{1} \kappa_{2}+1}{b_{\beta}+1}
$$

Assume that $u_{\mathrm{r}}, \delta n_{\mathrm{r}}$, and $\dot{\chi}_{\mathrm{r}}$ are constants, which makes (6.50) an LTI system.

We analyze closed-loop stability of the backstepping control system by finding eigenvalues of (6.50), which is populated using identified models shown in Table 5.5 and control gains, tuned through experimental and simulated trials, in Table 6.4. Table 6.3 shows the system eigenvalues for a range of operating conditions. All eigenvalues are in the left half plane, indicating exponential stability of the LTI system (6.50). Note that 
the eigenvalues are dependent upon $u_{\mathrm{r}}$ and $\dot{\chi}_{\mathrm{r}}$. The nominal reference course rate $\dot{\chi}_{\mathrm{r}}=0$ is used. Only five eigenvalues exist for higher speeds since the Nomoto steering model is used at these speeds.

Comparing Tables 6.3 and 6.2 one can see that the backstepping control law has eigenvalues that are considerably more negative than the PD control law, indicating that the backstepping control law has faster convergence than the PD control law. These more negative eigenvalues are partly due to (6.50) having three more nonzero terms than (6.30). (6.30) has a total of 6 gains for each speed region that can be adjusted to affect the system's eigenvalues, but (6.50), however, has 9 gains that cover the full speed envelope.

Table 6.3: Ribcraft USV Closed-Loop Linearized Backstepping Eigenvalues

\begin{tabular}{c|cccccc}
$u_{\mathrm{r}}(\mathrm{m} / \mathrm{s})$ & $\lambda_{1}$ & $\lambda_{2}$ & $\lambda_{3}$ & $\lambda_{4}$ & $\lambda_{5}$ & $\lambda_{6}$ \\
\hline 1.1 & -19.95 & -1.26 & -1.00 & -0.24 & -0.18 & -0.05 \\
1.5 & -19.95 & -1.26 & -0.96 & -0.24 & -0.15 & -0.09 \\
2.6 & -19.95 & -1.26 & -0.60 & -0.45 & -0.24 & $*$ \\
3.0 & -19.95 & -1.26 & $-0.53+0.29 \imath$ & $-0.53-0.29 \imath$ & -0.24 & \\
3.2 & -19.87 & -1.26 & $-0.53+0.37 \imath$ & $-0.53-0.37 \imath$ & -0.24 & \\
4.0 & -19.95 & -1.26 & $-0.53+0.58 \imath$ & $-0.53-0.58 \imath$ & -0.24 & \\
7.6 & -19.95 & $-0.53+1.43 \imath$ & $-0.53-1.43 \imath$ & -1.26 & -0.24 & \\
9.2 & -19.95 & $-0.53+1.77 \imath$ & $-0.53-1.77 \imath$ & -1.26 & -0.24 &
\end{tabular}

Table 6.4: Ribcraft USV Linearized Backstepping Control Gains

\begin{tabular}{cccccccc}
$\kappa_{1}$ & $\kappa_{2}$ & $\kappa_{3}$ & $\kappa_{4}$ & $\kappa_{5}$ & $k_{1}$ & $k_{2}$ & $k_{3}$ \\
\hline 1 & 1 & 1 & 1 & .2 & 20 & .1 & .5
\end{tabular}

Control gains in Table 6.4 were chosen to accommodate actuator rate limiting and saturation. Adjusting $\kappa_{1}$ and $\kappa_{2}$ changes how aggressively the vessel points to the desired position. Increasing $\kappa_{5}$ is analogous to increasing the "look ahead" distance in a cross track controller. Increasing $\kappa_{3}$ and $\kappa_{4}$ increases how aggressively the vessel speeds to the desired position.

\subsubsection{Incorporating Model Parameter Scheduling in Backstepping Control}

The results in Chapter 5 show that linear speed and steering models, with a speed-scheduled set of model parameters, adequately describe the dynamics of the Ribcraft USV. Earlier formulations of the backstepping 
control law in Section 6.3 assume that a single set of model parameters is used. The backstepping control law must be modified to include a speed scheduled set of model parameters. First, the control law must be augmented to account for discrepancies between parameters for the actual vessel state and for the virtual vehicle state. Secondly, the control law must additionally be changed to ensure that the Lyapunov function used to prove stability is smooth.

\subsubsection{Discrepancies in Parameters}

The model parameters used to create the reference trajectory switch values when the reference speed varies significantly. The vessel's actual speed and the reference speed may fall into different regimes, corresponding to different model parameter values. The trajectory tracking control law must account for these disparities in parameters.

Let the subscript " $r$ " denote parameter values associated with the reference trajectory at a given instant;

parameters without this subscript correspond to the vessel's actual speed. Differentiating (6.12-6.15), we have

$$
\begin{aligned}
\dot{e}_{u}=a_{u} e_{u}+\tilde{T}_{\mathrm{r}}-\tilde{T}+\Delta a_{u} u_{\mathrm{r}}+\Delta b_{u_{\text {bias }}} \\
\left(\begin{array}{c}
\dot{e}_{\chi} \\
\dot{e}_{\psi} \\
\ddot{e}_{\psi}
\end{array}\right)=\left(\begin{array}{ccc}
a_{\beta} & -a_{\beta} & 1+b_{\beta} \\
0 & 0 & 1 \\
0 & 0 & a_{r}
\end{array}\right)\left(\begin{array}{c}
e_{\chi} \\
e_{\psi} \\
\dot{e}_{\psi}
\end{array}\right)+\left(\begin{array}{c}
0 \\
0 \\
b_{r}
\end{array}\right)\left(\tilde{T}_{\mathrm{r}} \delta s_{\mathrm{r}}-\tilde{T} \delta s\right) \\
+\left(\begin{array}{c}
\Delta a_{\beta} \chi_{\mathrm{r}}-\Delta a_{\beta} \psi_{\mathrm{r}}+\Delta b_{\beta} \dot{\psi}_{\mathrm{r}}+\Delta b_{\beta_{\text {bias }}} \\
0 \\
\Delta a_{r} \dot{\psi}_{\mathrm{r}}+\Delta b_{r} \tilde{T}_{\mathrm{r}} \delta s_{\mathrm{r}}+\Delta b_{r_{\text {bias }}}
\end{array}\right)
\end{aligned}
$$


where

$$
\begin{aligned}
\Delta a_{u} & =a_{u_{\mathrm{r}}}-a_{u} \\
\Delta b_{u_{\mathrm{bias}}} & =b_{u_{\mathrm{bias}}}-b_{u_{\mathrm{bias}}} \\
\Delta b_{\beta_{\mathrm{bias}}} & =b_{\beta_{\mathrm{biasr}}}-b_{\beta_{\mathrm{bias}}} \\
\Delta a_{\beta} & =a_{\beta_{\mathrm{r}}}-a_{\beta} \\
\Delta b_{\beta} & =b_{\beta_{\mathrm{r}}}-b_{\beta} \\
\Delta a_{r} & =a_{r_{\mathrm{r}}}-a_{r} \\
\Delta b_{r} & =b_{r_{\mathrm{r}}}-b_{r} \\
\Delta b_{r_{\text {bias }}} & =b_{r_{\mathrm{bias}}}-b_{r_{\text {bias }}}
\end{aligned}
$$

The change of variables

$$
\left(\begin{array}{c}
\boldsymbol{\eta} \\
\xi \\
e_{\psi}
\end{array}\right)=\left(\begin{array}{c}
\eta_{1} \\
\eta_{2} \\
\eta_{3} \\
\xi_{1} \\
\xi_{2} \\
e_{\psi}
\end{array}\right)=\left(\begin{array}{c}
e_{s} \\
e_{y} \\
e_{\chi} \\
-a_{\beta} e_{\psi}+\left(b_{\beta}+1\right) \dot{e}_{\psi}+\left(\Delta a_{\beta} \chi_{\mathrm{r}}-\Delta a_{\beta} \psi_{\mathrm{r}}+\Delta b_{\beta} \dot{\psi}_{\mathrm{r}}+\Delta b_{\beta_{\mathrm{bias}}}\right) \\
e_{u} \\
e_{\psi}
\end{array}\right)
$$


gives the transformed trajectory tracking error dynamics

$$
\begin{aligned}
\dot{\eta}_{1}= & \left(\dot{\chi}_{\mathrm{r}}-a_{\beta} \eta_{3}+\xi_{1}\right) \eta_{2}-u_{\mathrm{r}}+\xi_{2}+u_{\mathrm{r}} \cos \eta_{3} \\
\dot{\eta}_{2}= & -\left(\dot{\chi}_{\mathrm{r}}-a_{\beta} \eta_{3}+\xi_{1}\right) \eta_{1}+u_{\mathrm{r}} \sin \eta_{3} \\
\dot{\eta}_{3}= & a_{\beta} \eta_{3}+\xi_{1} \\
\dot{\xi}_{1}= & \left(\frac{a_{r}\left(b_{\beta}+1\right)-a_{\beta}}{1+b_{\beta}}\right) \xi_{1}+\left(\frac{\left(a_{r}\left(b_{\beta}+1\right)-a_{\beta}\right) a_{\beta}}{1+b_{\beta}}\right) e_{\psi}+b_{r}\left(b_{\beta}+1\right)\left(\tilde{T}_{\mathrm{r}} \delta s_{\mathrm{r}}-\tilde{T} \delta s\right) \\
& +\left(1+b_{\beta}\right)\left(\Delta a_{r} \dot{\psi}_{\mathrm{r}}+b_{r_{\mathrm{r}}} T_{\mathrm{r}} \delta s_{\mathrm{r}}+\Delta b_{r_{\mathrm{bias}}}\right)-\frac{\left(a_{r}\left(1+b_{\beta}\right)-a_{\beta}\right)\left(\Delta a_{\beta} \chi_{\mathrm{r}}-\Delta a_{\beta} \psi_{\mathrm{r}}+\Delta b_{\beta} \dot{\psi}_{\mathrm{r}}+\Delta b_{\beta_{\text {bias }}}\right)}{1+b_{\beta}} \\
& +\left(\Delta a_{\beta} \dot{\chi}_{r}-\Delta a_{\beta} \dot{\psi}_{r}+\Delta b_{\beta} \ddot{\psi}_{r}\right) \\
\dot{\xi}_{2}= & a_{u} \xi_{2}+\tilde{T}_{\mathrm{r}}-\tilde{T}+\Delta a_{u} u_{\mathrm{r}}+\Delta b_{u_{\mathrm{bias}}} \\
\left(\begin{array}{c}
\dot{e}_{\psi} \\
\ddot{e}_{\psi}
\end{array}\right)= & \left(\begin{array}{cc}
0 & 1 \\
0 & a_{r}
\end{array}\right)\left(\begin{array}{c}
e_{\psi} \\
\dot{e}_{\psi}
\end{array}\right)+\left(\begin{array}{c}
0 \\
b_{r}
\end{array}\right)\left(\tilde{T}_{\mathrm{r}} \delta s_{\mathrm{r}}-\tilde{T} \delta s\right)+\left(\begin{array}{c}
\Delta a_{r} \dot{\psi}_{\mathrm{r}}+\Delta b_{r} \tilde{T}_{\mathrm{r}} \delta s_{\mathrm{r}}+\Delta b_{r_{\mathrm{bias}}}
\end{array}\right)
\end{aligned}
$$

Compare with the original backstepping error dynamics (6.34-6.39).

Augmenting the original control to accommodate the discrepancy in actual and reference model parame- 
ters simply requires adjusting the outer loop steering and speed dynamics as follows:

$$
\begin{aligned}
\left(\begin{array}{c}
\tilde{T} \delta s \\
\tilde{T}
\end{array}\right)= & \left(\begin{array}{c}
\tilde{T}_{\mathrm{r}} \delta s_{\mathrm{r}} \\
\tilde{T}_{\mathrm{r}}
\end{array}\right)+\left(\begin{array}{cc}
\frac{1}{b_{r}\left(b_{\beta}+1\right)} & 0 \\
0 & 1
\end{array}\right)\left(\left(\begin{array}{c}
\frac{a_{r}\left(b_{\beta}+1\right)-a_{\beta}}{1+b_{\beta}} \xi_{1}+\frac{\left(a_{r}\left(b_{\beta}+1\right)-a_{\beta}\right) a_{\beta}}{1+b_{\beta}} e_{\psi} \\
a_{u} \xi_{2}
\end{array}\right)\right. \\
& +\left(\begin{array}{c}
\left(1+b_{\beta}\right)\left(\Delta a_{r} \dot{\psi}_{\mathrm{r}}+b_{r_{\mathrm{r}}} T_{\mathrm{r}} \delta s_{\mathrm{r}}+\Delta b_{r_{\mathrm{bias}}}\right)-\frac{\left(a_{r}\left(1+b_{\beta}\right)-a_{\beta}\right)\left(\Delta a_{\beta} \chi_{\mathrm{r}}-\Delta a_{\beta} \psi_{\mathrm{r}}+\Delta b_{\beta} \dot{\psi}_{\mathrm{r}}+\Delta b_{\beta_{\mathrm{bias}}}\right.}{1+b_{\beta}} \\
\Delta a_{u} u_{\mathrm{r}}+\Delta b_{u_{\mathrm{bias}}}
\end{array}\right) \\
& +\left(\begin{array}{c}
\left(\Delta a_{\beta} \dot{\chi}_{r}-\Delta a_{\beta} \dot{\psi}_{r}+\Delta b_{\beta} \ddot{\psi}_{r}\right) \\
0
\end{array}\right) \\
& -\left(\begin{array}{c}
\left(-\int_{0}^{1} \cos \left(s \eta_{3}\right) d s \kappa_{5} u_{\mathrm{r}}\right)\left(-\left(\dot{\chi}_{\mathrm{r}}-a_{\beta} \eta_{3}+\xi_{1}\right) \eta_{1}+\kappa_{5} u_{\mathrm{r}} \sin \eta_{3}\right) \\
-\kappa_{3} \kappa_{4} \operatorname{sech}^{2}\left(\kappa_{4} \eta_{1}\right)\left(\left(\dot{\chi}_{\mathrm{r}}-a_{\beta} \eta_{3}+\xi_{1}\right) \eta_{2}+\kappa_{5}\left(-u_{\mathrm{r}}+u_{\mathrm{r}} \cos \eta_{3}+\xi_{2}\right)\right)
\end{array}\right) \\
& \left.+\left(\begin{array}{c}
\left.\left(-\int_{0}^{1} \sin _{3}\left(s \eta_{3}\right) s d s \eta_{2} \kappa_{5} u_{\mathrm{r}}-\kappa_{1} \kappa_{2} \operatorname{sech}^{2}\left(\kappa_{2} \eta_{3}\right)-a_{\beta}\right)\left(a_{\beta} \eta_{3}+\xi_{1}\right)\right) \\
u_{\mathrm{r}} \sin \eta_{3}\left(a_{\beta} \eta_{3}+\xi_{1}\right)
\end{array}\right)+\boldsymbol{K}\left(\begin{array}{c}
\xi_{1}+\left(\int_{0}^{1} \cos \left(s \eta_{3}\right) d s\right) \eta_{2} \kappa_{5} u_{\mathrm{r}}+\kappa_{1} \tanh \left(\kappa_{2} \eta_{3}\right)+a_{\beta} \eta_{3} \\
\eta_{2}-u_{\mathrm{r}}+u_{\mathrm{r}} \cos \eta_{3}+\kappa_{3} \tanh \left(\kappa_{4} \eta_{1}\right)
\end{array}\right)\right)
\end{aligned}
$$

This modified control law cancels the additional terms. Closed-loop stability properties follow as for (6.46).

Proposition 6.3.4. The control law (6.58) globally asymptotically stabilizes the dynamic system (6.52-6.56) about the equilibrium $(\boldsymbol{\eta}, \boldsymbol{\xi})=(\mathbf{0}, \mathbf{0})$ and $e_{\psi}$ remains bounded and converges to zero, provided $\beta_{\mathrm{r}}, \chi_{\mathrm{r}}, \delta s_{\mathrm{r}}$, $u_{\mathrm{r}}$, and $\delta n_{\mathrm{r}}$ are bounded and $\tilde{T} \neq 0$.

The proof of Proposition 6.3.4 follows similarly to the proof of Proposition 6.3.3.

\subsubsection{Discontinuities in the Lyapunov Function}

Without a smooth Lyapunov function Proposition 6.3.4 only proves stability if parameters never switch, which is an impractical assumption. To regain global asymptotic stability of the parameter scheduled vessel dynamics we expand upon method shown in [Liberzon, 2003] requiring one or more Lyapunov functions for each switched subsystem. Each Lyapunov function must prove asymptotic stability of its associated subsystem as well as maintain continuity along the switching manifolds between adjacent subsystems' Lyapunov 
functions.

Assume there exists a set of Lyapunov functions

$$
\left\{V^{(i)}=\frac{1}{2} \eta_{1}^{2}+\frac{1}{2} \eta_{2}^{2}+\frac{1}{2} \eta_{3}^{2}+\frac{1}{2}(\boldsymbol{\xi}-\boldsymbol{\phi}(\boldsymbol{\eta}))^{T} \boldsymbol{H}^{(i)}(\boldsymbol{\xi}-\boldsymbol{\phi}(\boldsymbol{\eta}))>0 \quad \mid i=1,2,3, \ldots\right\}
$$

where $\boldsymbol{H}^{(i)}$ is a constant, positive, diagonal $2 \times 2$ matrix

$$
\boldsymbol{H}^{(i)}=\left(\begin{array}{cc}
h_{1}^{(i)} & 0 \\
0 & 1
\end{array}\right)
$$

The superscript $(i)$ found in the function $V^{(i)}$ and matrix $\boldsymbol{H}^{(i)}$ correspond to the current continuous subsystem. Proper definition of the $\boldsymbol{H}^{(i+1)}$ (indicating the next continuous subsystem) guarantees that switching between $V^{(i)}$ and $V^{(i+1)}$ is smooth. The switching manifolds are defined by the actual and reference vessels' forward velocity. A Lyapunov function exists for every instance that the reference or estimated vessel parameters switch. Specifically,

$$
h_{1}^{(i+1)}=h_{1}^{(i)}\left(\frac{\xi_{1}^{(i)}-\phi_{1}^{(i)}(\boldsymbol{\eta})}{\xi_{1}^{(i)}-\phi_{1}^{(i)}(\boldsymbol{\eta})-\Delta a_{\beta}^{*} e_{\psi}+\Delta b_{\beta}^{*} \dot{e}_{\psi}+\left(-\Delta a_{\beta}^{*} \chi_{\mathrm{r}}+\Delta a_{\beta}^{*} \psi_{\mathrm{r}}-\Delta b_{\beta}^{*} \dot{\psi}_{\mathrm{r}}-\Delta b_{\beta_{\mathrm{bias}}^{*}}^{*}\right)-\Delta a_{\beta}^{*} \eta_{3}}\right)^{2}
$$

when the actual vessel's parameters switch and

$$
h_{1}^{(i+1)}=h_{1}^{(i)}\left(\frac{\xi_{1}^{(i)}-\phi_{1}^{(i)}(\boldsymbol{\eta})}{\xi_{1}^{(i)}-\phi_{1}^{(i)}(\boldsymbol{\eta})+\left(\Delta a_{\beta_{\mathrm{r}}}^{*} \chi_{\mathrm{r}}-\Delta a_{\beta_{\mathrm{r}}}^{*} \psi_{\mathrm{r}}+\Delta b_{\beta_{\mathrm{r}}}^{*} \dot{\psi}_{\mathrm{r}}+\Delta b_{\beta_{\mathrm{bias}}}^{*}\right)}\right)^{2}
$$

when the reference vessel's parameters switch. Like for Lyapunov function $V$ and matrix $\boldsymbol{H}$ the $\xi_{1}$ and $\phi_{1}$ 
with the superscripts $(i)$ and $(i+1)$ correspond to the current and next continuous subsystems. Note that

$$
\begin{gathered}
\Delta a_{\beta}^{*}=a_{\beta}^{(i+1)}-a_{\beta}^{(i)} \\
\Delta a_{\beta_{\mathrm{r}}}^{*}=a_{\beta_{\mathrm{r}}}^{(i+1)}-a_{\beta_{\mathrm{r}}}^{(i)} \\
\Delta b_{\beta}^{*}=b_{\beta}^{(i+1)}-b_{\beta}^{(i)} \\
\Delta b_{\beta_{\mathrm{r}}}^{*}=b_{\beta_{\mathrm{r}}}^{(i+1)}-b_{\beta_{\mathrm{r}}}^{(i)} \\
\Delta b_{\beta_{\text {bias }}^{*}}^{*}=b_{\beta_{\text {bias }}}^{(i+1)}-b_{\beta_{\text {bias }}}^{(i)} \\
\Delta b_{\beta_{\text {biasr }}^{*}}=b_{\beta_{\text {biasr }}}^{(i+1)}-b_{\beta_{\text {biasr }}}^{(i)}
\end{gathered}
$$

By using (6.60) and (6.61) continuity is maintained between Lyapunov functions. Furthermore if $\boldsymbol{H}^{(1)}$ is positive and diagonal all successive matrices $\left\{\boldsymbol{H}^{(i)} \mid i=2,3,4, \ldots\right\}$ will be positive and diagonal provided a switch in $a_{\beta}$ and $b_{\beta}$ parameters does not cause $\xi_{1}-\phi_{1}(\boldsymbol{\eta}) \rightarrow 0$.

Proposition 6.3.5. If $\boldsymbol{H}^{(1)}$ is positive and diagonal then $\left\{\boldsymbol{H}^{(i)} \mid i=2,3,4, \ldots\right\}$ are positive and diagonal provided switching manifolds do not cause the discontinuous state $\xi_{1}$ to induce $\xi_{1}-\phi_{1}(\boldsymbol{\eta}) \rightarrow 0$ and $\xi_{1}-$ $\phi_{1}(\boldsymbol{\eta}) \neq 0$ at the time the parameters switch.

Proof: Looking at the non-zero terms of $\boldsymbol{H}^{(i+1)}$ during switches and assuming $\boldsymbol{H}^{(i)}$ is positive and diagonal we have

$$
h_{1}^{(i+1)}=h_{1}^{(i)} \Delta_{\text {vessel }}^{2}>0
$$

for vessel parameter switching and

$$
h_{1}^{(i+1)}=h_{1}^{(i)} \Delta_{\text {ref }}^{2}>0
$$

for reference trajectory parameter switching where

$$
\Delta_{\text {vessel }}=\frac{\xi_{1}^{(i)}-\phi_{1}^{(i)}(\boldsymbol{\eta})}{\xi_{1}^{(i)}-\phi_{1}^{(i)}(\boldsymbol{\eta})-\Delta a_{\beta}^{*} e_{\psi}+\Delta b_{\beta}^{*} \dot{e}_{\psi}+\left(-\Delta a_{\beta}^{*} \chi_{\mathrm{r}}+\Delta a_{\beta}^{*} \psi_{\mathrm{r}}-\Delta b_{\beta}^{*} \dot{\psi}_{\mathrm{r}}-\Delta b_{\beta_{\mathrm{bias}}}\right)-\Delta a_{\beta}^{*} \eta_{3}}
$$


and

$$
\Delta_{\mathrm{ref}}=\frac{\xi_{1}^{(i)}-\phi_{1}^{(i)}(\boldsymbol{\eta})}{\xi_{1}^{(i)}-\phi_{1}^{(i)}(\boldsymbol{\eta})+\left(\Delta a_{\beta_{\mathrm{r}}}^{*} \chi_{\mathrm{r}}-\Delta a_{\beta_{\mathrm{r}}}^{*} \psi_{\mathrm{r}}+\Delta b_{\beta_{\mathrm{r}}}^{*} \dot{\psi}_{\mathrm{r}}+\Delta b_{\beta_{\mathrm{bias}_{\mathrm{r}}}^{*}}\right)}
$$

Since it is assumed that $\boldsymbol{H}^{(1)}$ is positive and diagonal and between any step $\boldsymbol{H}^{(i)} \rightarrow \boldsymbol{H}^{(i+1)}>0$ then $\boldsymbol{H}^{(i)}$ remains positive and diagonal for all values of $(i)$ by induction.

Unlike the standard backstepping technique the Lyapunov functions (6.59) contains a positive, diagonal matrix $\boldsymbol{H}$. The traditional multivariable backstepping technique employs the control law

$$
\boldsymbol{u}=\boldsymbol{G}_{a}^{-1}(\boldsymbol{\eta}, \boldsymbol{\xi})\left(-\boldsymbol{f}_{a}(\boldsymbol{\eta}, \boldsymbol{\xi})+\frac{\partial \phi}{\partial \boldsymbol{\eta}}(\boldsymbol{f}(\boldsymbol{\eta})+\boldsymbol{G}(\boldsymbol{\eta}) \boldsymbol{\xi})-\left(\frac{\partial V}{\partial \boldsymbol{\eta}} \boldsymbol{G}(\boldsymbol{\eta})\right)^{T}-\boldsymbol{K}(\boldsymbol{\xi}-\boldsymbol{\phi}(\boldsymbol{\eta}))\right)
$$

for the Lyapunov function

$$
V_{a}(\boldsymbol{\eta}, \boldsymbol{\xi})=V(\boldsymbol{\eta})+\frac{1}{2}(\boldsymbol{\xi}-\boldsymbol{\phi}(\boldsymbol{\eta}))^{T}(\boldsymbol{\xi}-\boldsymbol{\phi}(\boldsymbol{\eta}))
$$

where $V(\boldsymbol{\eta})>0$ and $\dot{V} \leq-W(\boldsymbol{\eta})$ for a positive definite function $W$ yielding a Lyapunov rate

$$
\begin{aligned}
\dot{V}_{a}= & \frac{\partial V}{\partial \boldsymbol{\eta}}(\boldsymbol{f}(\boldsymbol{\eta})+\boldsymbol{G}(\boldsymbol{\eta}) \boldsymbol{\xi})+(\boldsymbol{\xi}-\boldsymbol{\phi}(\boldsymbol{\eta}))^{T}\left(\dot{\boldsymbol{\xi}}-\frac{\partial \phi}{\partial \boldsymbol{\eta}} \dot{\boldsymbol{\eta}}\right) \\
= & \frac{\partial V}{\partial \boldsymbol{\eta}}(\boldsymbol{f}(\boldsymbol{\eta})+\boldsymbol{G}(\boldsymbol{\eta}) \boldsymbol{\phi}(\boldsymbol{\eta}))+\frac{\partial V}{\partial \boldsymbol{\eta}} \boldsymbol{G}(\boldsymbol{\eta})(\boldsymbol{\xi}-\boldsymbol{\phi}(\boldsymbol{\eta})) \\
& +(\boldsymbol{\xi}-\boldsymbol{\phi}(\boldsymbol{\eta}))^{T}\left(\boldsymbol{f}_{a}(\boldsymbol{\eta}, \boldsymbol{\xi})+\boldsymbol{G}_{a}(\boldsymbol{\eta}, \boldsymbol{\xi}) \boldsymbol{u}-\frac{\partial \phi}{\partial \boldsymbol{\eta}}(\boldsymbol{f}(\boldsymbol{\eta})+\boldsymbol{G}(\boldsymbol{\eta}) \boldsymbol{\xi})\right) \\
\leq & -W(\boldsymbol{\eta})-(\boldsymbol{\xi}-\boldsymbol{\phi}(\boldsymbol{\eta}))^{T} \boldsymbol{K}(\boldsymbol{\xi}-\boldsymbol{\phi}(\boldsymbol{\eta}))<0
\end{aligned}
$$

assuming $\boldsymbol{K}$ is positive definite, proving asymptotic stability of the equilibrium $(\boldsymbol{\eta}, \boldsymbol{\xi})=(\mathbf{0}, \mathbf{0})$ of the system

$$
\begin{aligned}
& \dot{\boldsymbol{\eta}}=\boldsymbol{f}(\boldsymbol{\eta})+\boldsymbol{G}(\boldsymbol{\eta}) \boldsymbol{\xi} \\
& \dot{\boldsymbol{\xi}}=\boldsymbol{f}_{a}(\boldsymbol{\eta}, \boldsymbol{\xi})+\boldsymbol{G}_{a}(\boldsymbol{\eta}, \boldsymbol{\xi}) \boldsymbol{u}
\end{aligned}
$$


Including a positive diagonal $\boldsymbol{H}$ we have the control law

$$
\boldsymbol{u}=\boldsymbol{G}_{a}^{-1}(\boldsymbol{\eta}, \boldsymbol{\xi})\left(-\boldsymbol{f}_{a}(\boldsymbol{\eta}, \boldsymbol{\xi})+\frac{\partial \phi}{\partial \boldsymbol{\eta}}(\boldsymbol{f}(\boldsymbol{\eta})+\boldsymbol{G}(\boldsymbol{\eta}) \boldsymbol{\xi})-\boldsymbol{H}^{-1}\left(\frac{\partial V}{\partial \boldsymbol{\eta}} \boldsymbol{G}(\boldsymbol{\eta})\right)^{T}-\boldsymbol{K}(\boldsymbol{\xi}-\boldsymbol{\phi}(\boldsymbol{\eta}))\right)
$$

for the Lyapunov function

$$
V_{a}(\boldsymbol{\eta}, \boldsymbol{\xi})=V(\boldsymbol{\eta})+\frac{1}{2}(\boldsymbol{\xi}-\boldsymbol{\phi}(\boldsymbol{\eta}))^{T} \boldsymbol{H}(\boldsymbol{\xi}-\boldsymbol{\phi}(\boldsymbol{\eta}))
$$

which gives the Lyapunov rate

$$
\begin{aligned}
\dot{V}_{a}= & \frac{\partial V}{\partial \boldsymbol{\eta}}(\boldsymbol{f}(\boldsymbol{\eta})+\boldsymbol{G}(\boldsymbol{\eta}) \boldsymbol{\xi})+(\boldsymbol{\xi}-\boldsymbol{\phi}(\boldsymbol{\eta}))^{T} \boldsymbol{H}\left(\dot{\boldsymbol{\xi}}-\frac{\partial \phi}{\partial \boldsymbol{\eta}} \dot{\boldsymbol{\eta}}\right) \\
= & \frac{\partial V}{\partial \boldsymbol{\eta}}(\boldsymbol{f}(\boldsymbol{\eta})+\boldsymbol{G}(\boldsymbol{\eta}) \boldsymbol{\phi}(\boldsymbol{\eta}))+\frac{\partial V}{\partial \boldsymbol{\eta}} \boldsymbol{G}(\boldsymbol{\eta})(\boldsymbol{\xi}-\boldsymbol{\phi}(\boldsymbol{\eta})) \\
& +(\boldsymbol{\xi}-\boldsymbol{\phi}(\boldsymbol{\eta}))^{T} \boldsymbol{H}\left(\boldsymbol{f}_{a}(\boldsymbol{\eta}, \boldsymbol{\xi})+\boldsymbol{G}_{a}(\boldsymbol{\eta}, \boldsymbol{\xi}) \boldsymbol{u}-\frac{\partial \phi}{\partial \boldsymbol{\eta}}(\boldsymbol{f}(\boldsymbol{\eta})+\boldsymbol{G}(\boldsymbol{\eta}) \boldsymbol{\xi})\right) \\
\leq & -W(\boldsymbol{\eta})-(\boldsymbol{\xi}-\boldsymbol{\phi}(\boldsymbol{\eta}))^{T} \boldsymbol{K} \boldsymbol{H}(\boldsymbol{\xi}-\boldsymbol{\phi}(\boldsymbol{\eta}))<0
\end{aligned}
$$

proving asymptotic stability of the equilibrium $(\boldsymbol{\eta}, \boldsymbol{\xi})=(\mathbf{0}, \mathbf{0})$ for the system defined by (6.62) and (6.63) as long as $\boldsymbol{K}$ is restricted to being positive and diagonal.

Assume a set of positive diagonal matrices $\boldsymbol{H}^{(i)}$ exists, giving a set of Lyapunov functions $V_{a}^{(i)}(\boldsymbol{\eta}, \boldsymbol{\xi})$ defined by (6.59) that are continuous on switching manifolds for systems (6.62) and (6.63) where $(\boldsymbol{\xi}-\boldsymbol{\phi}(\boldsymbol{\eta}))$ switches between subsystems. Using $\boldsymbol{H}^{(i)}$ and a positive diagonal $\boldsymbol{K}$ in (6.64) will then globally asymptotic stabilize the equilibrium $(\boldsymbol{\eta}, \boldsymbol{\xi})=(\mathbf{0}, \mathbf{0})$ for $(6.62)$ and (6.63). 
Using the knowledge gained from using the Lyapunov function (6.65) we set the control law to

$$
\begin{aligned}
& \left(\begin{array}{c}
\tilde{T} \delta s \\
\tilde{T}
\end{array}\right)=\left(\begin{array}{c}
\tilde{T}_{\mathrm{r}} \delta s_{\mathrm{r}} \\
\tilde{T}_{\mathrm{r}}
\end{array}\right)+\left(\begin{array}{cc}
\frac{1}{b_{r}\left(b_{\beta}+1\right)} & 0 \\
0 & 1
\end{array}\right)\left(\left(\begin{array}{c}
\frac{a_{r}\left(b_{\beta}+1\right)-a_{\beta}}{1+b_{\beta}} \xi_{1}+\frac{\left(a_{r}\left(b_{\beta}+1\right)-a_{\beta}\right) a_{\beta}}{1+b_{\beta}} e_{\psi} \\
a_{u} \xi_{2}
\end{array}\right)\right. \\
& +\left(\begin{array}{c}
\left(1+b_{\beta}\right)\left(\Delta a_{r} \dot{\psi}_{\mathrm{r}}+b_{r_{\mathrm{r}}} T_{\mathrm{r}} \delta s_{\mathrm{r}}+\Delta b_{r_{\mathrm{bias}}}\right)-\frac{\left(a_{r}\left(1+b_{\beta}\right)-a_{\beta}\right)\left(\Delta a_{\beta} \chi_{\mathrm{r}}-\Delta a_{\beta} \psi_{\mathrm{r}}+\Delta b_{\beta} \dot{\psi}_{\mathrm{r}}+\Delta b_{\beta_{\mathrm{bias}}}\right.}{1+b_{\beta}} \\
\Delta a_{u} u_{\mathrm{r}}+\Delta b_{u_{\text {bias }}}
\end{array}\right) \\
& +\left(\begin{array}{c}
\left(\Delta a_{\beta} \dot{\chi}_{r}-\Delta a_{\beta} \dot{\psi}_{r}+\Delta b_{\beta} \ddot{\psi}_{r}\right) \\
0
\end{array}\right) \\
& -\left(\begin{array}{c}
\left(-\int_{0}^{1} \cos \left(s \eta_{3}\right) d s \kappa_{5} u_{\mathrm{r}}\right)\left(-\left(\dot{\chi}_{\mathrm{r}}-a_{\beta} \eta_{3}+\xi_{1}\right) \eta_{1}+\kappa_{5} u_{\mathrm{r}} \sin \eta_{3}\right) \\
-\kappa_{3} \kappa_{4} \operatorname{sech}^{2}\left(\kappa_{4} \eta_{1}\right)\left(\left(\dot{\chi}_{\mathrm{r}}-a_{\beta} \eta_{3}+\xi_{1}\right) \eta_{2}+\kappa_{5}\left(-u_{\mathrm{r}}+u_{\mathrm{r}} \cos \eta_{3}+\xi_{2}\right)\right)
\end{array}\right) \\
& -\left(\begin{array}{c}
\left.\left(-\int_{0}^{1} \sin \left(s \eta_{3}\right) s d s \eta_{2} \kappa_{5} u_{\mathrm{r}}-\kappa_{1} \kappa_{2} \operatorname{sech}^{2}\left(\kappa_{2} \eta_{3}\right)-a_{\beta}\right)\left(a_{\beta} \eta_{3}+\xi_{1}\right)\right) \\
u_{\mathrm{r}} \sin \eta_{3}\left(a_{\beta} \eta_{3}+\xi_{1}\right)
\end{array}\right) \\
& \left.+\boldsymbol{H}^{(i)^{-1}}\left(\begin{array}{c}
\eta_{3} \\
\eta_{1}
\end{array}\right)+\boldsymbol{K}\left(\begin{array}{c}
\xi_{1}+\left(\int_{0}^{1} \cos \left(s \eta_{3}\right) d s\right) \eta_{2} \kappa_{5} u_{\mathrm{r}}+\kappa_{1} \tanh \left(\kappa_{2} \eta_{3}\right)+a_{\beta} \eta_{3} \\
\xi_{2}-u_{\mathrm{r}}+u_{\mathrm{r}} \cos \eta_{3}+\kappa_{3} \tanh \left(\kappa_{4} \eta_{1}\right)
\end{array}\right)\right)
\end{aligned}
$$

which asymptotically stabilizes the equilibrium $\boldsymbol{\eta}=\mathbf{0}$ and $\boldsymbol{\xi}=\mathbf{0}$ if $\beta_{\mathrm{r}}, \chi_{\mathrm{r}}, \delta s_{\mathrm{r}}, u_{\mathrm{r}}, \delta n_{\mathrm{r}}$ are bounded, the gain matrix

$$
\boldsymbol{K}=\left(\begin{array}{cc}
k_{1} & 0 \\
0 & k_{2}
\end{array}\right)
$$

is constant, positive, and diagonal, $\tilde{T} \neq 0$, and the set of matrices

$$
\boldsymbol{H}^{(i)}=\left(\begin{array}{cc}
h_{1}^{(i)} & 0 \\
0 & 1
\end{array}\right)
$$

are all constant, positive, and diagonal by conforming to (6.60) and (6.61).

Proposition 6.3.6. The control law (6.66) globally asymptotically stabilizes the dynamic system (6.34-6.38) about the equilibrium $\boldsymbol{\eta}=\mathbf{0}$ and $\boldsymbol{\xi}=\mathbf{0}$ and $e_{\psi}$ remains bounded and converges asymptotically to zero 
provided $\beta_{\mathrm{r}}, \chi_{\mathrm{r}}, \delta s_{\mathrm{r}}, u_{\mathrm{r}}$, and $\delta n_{\mathrm{r}}$ are bounded, $\tilde{T} \neq 0$, and $\frac{a_{\beta}}{1+b_{\beta}}<0$.

Proof: Consider the error dynamics in (6.34-6.38). Choose the set of radially unbounded candidate Lyapunov functions

$$
V_{2}^{(i)}=\frac{1}{2} \eta_{1}^{2}+\frac{1}{2} \eta_{2}^{2}+\frac{1}{2} \eta_{3}^{2}+\frac{1}{2}(\boldsymbol{\xi}-\phi(\boldsymbol{\eta}))^{T} \boldsymbol{H}^{(i)}(\boldsymbol{\xi}-\boldsymbol{\phi}(\boldsymbol{\eta}))>0
$$

where $\boldsymbol{H}^{(i)}$ is a positive diagonal matrix defined in (6.60) and (6.61), switches to a positive diagonal $\boldsymbol{H}^{(i+1)}$ such that subsequent Lyapunov functions are continuous along the switching manifold. Applying the control law (6.66), the time derivative of (6.67) is

$$
\dot{V}_{2}^{(i)}=-\kappa_{3} \eta_{1} \tanh \left(\kappa_{4} \eta_{1}\right)-\kappa_{1} \eta_{3} \tanh \left(\kappa_{1} \eta_{3}\right)-(\boldsymbol{\xi}-\boldsymbol{\phi}(\boldsymbol{\eta}))^{T} \boldsymbol{K} \boldsymbol{H}^{(i)}(\boldsymbol{\xi}-\boldsymbol{\phi}(\boldsymbol{\eta})) \leq 0
$$

Using Lemma 6.3.1 for the $\boldsymbol{\eta}$ subsystem, and recalling that $\boldsymbol{K}$ is a positive diagonal matrix and the positive diagonal $\boldsymbol{H}^{(i)}$ induces continuity of $(\boldsymbol{\xi}-\boldsymbol{\phi}(\boldsymbol{\eta})$ between switching manifolds, the equilibrium is globally asymptotically stable by Lyapunov's direct method, provided $e_{\psi}$ is bounded so that the input (6.66) remains well-defined. Boundedness of $e_{\psi}$ is shown in (6.3.3).

\subsubsection{Including Sternward Bifurcation Dynamics}

The control law (6.66) can be applied to sternward dynamics by carefully adjusting how the reference trajectory is generated and augmenting the stability proof to account for cases where actual and reference parameters never converge. We assume that a single stable model with a zero turn rate equilibrium is used to generate reference trajectories. This assumption simplifies the optimization approach used to generate feasible trajectories that avoid obstacles en route to a set goal. However, the parameter discrepancies noted in Section 6.3.1 will now exist even for the zero error case, requiring the stability properties to be reanalyzed.

If, for the zero error case, all parameter discrepancies are zero then Proposition 6.3.5 proves global asymptotic stability of the system. However, reference trajectories are generated using a stable turn rate model with an equilibrium about zero turn rate, but the estimated vessel is modeled by stable left and right turn steering models and an unstable zero turn rate model. 
Proposition 6.3.7. The control law (6.66) globally asymptotically stabilizes the dynamic system (6.34-6.38) about the equilibrium $\boldsymbol{\eta}=\mathbf{0}$ and $\boldsymbol{\xi}=\mathbf{0}$ and $e_{\psi}$ remains bounded and converges asymptotically to zero provided $\beta_{\mathrm{r}}, \chi_{\mathrm{r}}, \delta s_{\mathrm{r}}, u_{\mathrm{r}}$, and $\delta n_{\mathrm{r}}$ are bounded, $\tilde{T} \neq 0$, and $\frac{a_{\beta}}{1+b_{\beta}}<0$.

Proof: Consider the error dynamics in (6.34-6.38). Choose the set of radially unbounded candidate Lyapunov functions

$$
V_{2}^{(i)}=\frac{1}{2} \eta_{1}^{2}+\frac{1}{2} \eta_{2}^{2}+\frac{1}{2} \eta_{3}^{2}+\frac{1}{2}(\boldsymbol{\xi}-\boldsymbol{\phi}(\boldsymbol{\eta}))^{T} \boldsymbol{H}^{(i)}(\boldsymbol{\xi}-\boldsymbol{\phi}(\boldsymbol{\eta}))>0
$$

where $\boldsymbol{H}^{(i)}$ is a positive diagonal matrix defined in (6.60) and (6.61), switches to a positive diagonal $\boldsymbol{H}^{(i+1)}$ such that subsequent Lyapunov functions are continuous along the switching manifold. Applying the control law (6.66), the time derivative of (6.68) is

$$
\dot{V}_{2}^{(i)}=-\kappa_{3} \eta_{1} \tanh \left(\kappa_{4} \eta_{1}\right)-\kappa_{1} \eta_{3} \tanh \left(\kappa_{1} \eta_{3}\right)-(\boldsymbol{\xi}-\boldsymbol{\phi}(\boldsymbol{\eta}))^{T} \boldsymbol{K} \boldsymbol{H}^{(i)}(\boldsymbol{\xi}-\boldsymbol{\phi}(\boldsymbol{\eta})) \leq 0
$$

Using Lemma 6.3.1 for the $\boldsymbol{\eta}$ subsystem, and recalling that $\boldsymbol{K}$ is a positive diagonal matrix and the positive diagonal $\boldsymbol{H}^{(i)}$ induces continuity of $(\boldsymbol{\xi}-\boldsymbol{\phi}(\boldsymbol{\eta})$ between switching manifolds, the equilibrium is globally asymptotically stable by Lyapunov's direct method, provided $e_{\psi}$ and $\boldsymbol{H}^{(i)}$ are bounded so that the input (6.66) remains well-defined. Boundedness of $\boldsymbol{H}^{(i)}$ is shown in Proposition 6.3.5).

Rearranging the $\xi_{1}$ transform in (6.33) gives

$$
\dot{e}_{\psi}=\frac{a_{\beta}}{b_{\beta}+1} e_{\psi}+\frac{1}{b_{\beta}+1} \xi_{1}+\Delta a_{\beta} \chi_{\mathrm{r}}-\Delta a_{\beta} \psi_{\mathrm{r}}+\Delta b_{\beta} \dot{\psi}_{\mathrm{r}}+\Delta b_{\beta_{\text {bias }}}
$$

Since $\frac{a_{\beta}}{1+b_{\beta}}<0$, the system (6.49) is exponentially stable. Since $\xi_{1}$ remains bounded while the input (6.66) is well-defined and the " $\Delta$ " terms are finite, $e_{\psi}$ (and $\dot{e}_{\psi}$ ) remains bounded; see Lemma 4.6 in [Khalil, 2002]. Note that as $\xi_{1}$ converges to zero, $e_{\psi}$ and $\dot{e}_{\psi}$ remain bounded but do not converge to zero due to parameter discrepancies between the model used to generate trajectories and the models used to estimate actual vessel dynamics.

The result that the $e_{\psi}$ terms do not converge to zero for cases where reference and actual dynamics is 
intuitive. For only two actuators to stabilize three error terms requires that reference and actual systems match and that at least one of the error terms is stable. In our case the $\beta$ dynamics and consequently $\chi$ dynamics are stable as well as the $u$ dynamics, which allows for the terms in $\xi$ to be stabilized without regard for the particular heading of the boat.

\subsubsection{Including Steady and Slowly Varying Flow}

Vessels operating in riverine environments must contend with and compensate for fluid flow. Assuming that flow velocity varies slowly enough to be considered uniform and constant over the time scale of interest, we consider an inertial "flow frame" that moves at constant speed relative to the stationary inertial frame. Note that if the reference trajectory is generated with knowledge of this flow, the closed-loop system will behave identically to the case where there is no flow. The control law attenuates relative errors between the actual and reference vessel states. Suppose, however, that the reference trajectory is generated without knowledge of the flow.

Define the reference course angle and velocity (in the inertial frame) to now include flow terms giving

$$
\chi_{\mathrm{r}_{\text {new }}}=\beta_{\mathrm{r}_{\text {flow }}}+\chi_{\mathrm{r}}
$$

and

$$
u_{\mathrm{r}_{\text {new }}}=u_{\mathrm{r}_{\text {flow }}}+u_{\mathrm{r}}
$$

The additional terms $\beta_{\mathrm{r}_{\text {flow }}}$ and $u_{\mathrm{r}_{\text {flow }}}$ are functions of $\psi_{\mathrm{r}}, r_{\mathrm{r}}$, and $u_{\mathrm{r}}$ that modify the reference trajectory's original body states to drive the reference vessel to the original position in the presence of flow. Specifically,

$$
\beta_{\mathrm{r}_{\text {flow }}}=\tan ^{-1}\left(\frac{V_{\text {flow }} \sin \left(-\chi_{\mathrm{r}}+\psi_{\text {flow }}\right)}{u_{\mathrm{r}}+V_{\text {flow }} \cos \left(-\chi_{\mathrm{r}}+\psi_{\text {flow }}\right)}\right)
$$


and

$$
\begin{aligned}
u_{\mathrm{r}_{\text {flow }}}= & \left(\left(u_{\mathrm{r}}+V_{\text {flow }} \cos \left(-\chi_{\mathrm{r}}+\psi_{\text {flow }}\right)\right)^{2}\right. \\
& \left.+V_{\text {flow }}^{2} \sin ^{2}\left(-\chi_{\mathrm{r}}+\psi_{\text {flow }}\right)\right)^{\frac{1}{2}}-u_{\mathrm{r}}
\end{aligned}
$$

where $V_{\text {flow }}$ and $\psi_{\text {flow }}$ correspond to the magnitude and direction of the fluid flow. Recall that we are assuming $u \approx V$. Both (6.71) and (6.72) are bounded since $u_{\mathrm{r}}$ and $\chi_{\mathrm{r}}$ are bounded. Taking time derivatives of (6.71) and (6.72) (assuming these time derivatives are well-defined) yields

$$
\begin{gathered}
\dot{\beta}_{\mathrm{r}_{\text {flow }}}=-\frac{V_{\text {flow }}\left(\dot{u}_{\mathrm{r}} \sin \left(-\chi_{\mathrm{r}}+\psi_{\text {flow }}\right)\right)}{2 V_{\text {flow }} u_{\mathrm{r}} \cos \left(-\chi_{\mathrm{r}}+\psi_{\text {flow }}\right)+u_{\mathrm{r}}^{2}+V_{\text {flow }}^{2}} \\
+\frac{\dot{\chi}_{\mathrm{r}} V_{\text {flow }}\left(u_{\mathrm{r}} \cos \left(-\chi_{\mathrm{r}}+\psi_{\text {flow }}\right)+V_{\text {flow }}\right)}{2 V_{\text {flow }} u_{\mathrm{r}} \cos \left(-\chi_{\mathrm{r}}+\psi_{\text {flow }}\right)+u_{\mathrm{r}}^{2}+V_{\text {flow }}^{2}}
\end{gathered}
$$

and

$$
\begin{gathered}
\dot{u}_{\mathrm{r}_{\text {flow }}}=\frac{\dot{u}_{\mathrm{r}}\left(u_{\mathrm{r}}+V_{\text {flow }} \cos \left(-\chi_{\mathrm{r}}+\psi_{\text {flow }}\right)\right)}{\sqrt{2 V_{\text {flow }} u_{\mathrm{r}} \cos \left(-\chi_{\mathrm{r}}+\psi_{\text {flow }}\right)+u_{\mathrm{r}}^{2}+V_{\text {flow }}^{2}}} \\
+\frac{V_{\text {flow }} u_{\mathrm{r}}\left(\dot{\chi}_{\mathrm{r}}\right) \sin \left(-\chi_{\mathrm{r}}+\psi_{\text {flow }}\right)}{\sqrt{2 V_{\text {flow }} u_{\mathrm{r}} \cos \left(-\chi_{\mathrm{r}}+\psi_{\text {flow }}\right)+u_{\mathrm{r}}^{2}+V_{\text {flow }}^{2}}} \\
-\dot{u}_{\mathrm{r}}
\end{gathered}
$$

The new reference variables $\chi_{\mathrm{r}_{\text {new }}}$ and $u_{\mathrm{r}_{\text {new }}}$ replace the original $\chi_{\mathrm{r}}$ and $u_{\mathrm{r}}$ terms, however, the speed and course reference model parameters do not reflect this change. Using (6.73) and (6.74) we modify the speed bias $b_{u_{\text {biasr }}}$ and course bias $b_{\beta_{\text {biasr }}}$ causing the reference models to match their augmented speed and course 
states. Specifically,

$$
\begin{aligned}
b_{\beta_{\text {bias }}}= & \dot{\chi}_{\mathrm{r}_{\text {new }}}-a_{\beta_{\mathrm{r}}} \chi_{\mathrm{r}_{\text {new }}}+a_{\beta_{\mathrm{r}}} \psi_{\mathrm{r}}-\left(1+b_{\beta_{\mathrm{r}}}\right) r_{\mathrm{r}} \\
= & \frac{V_{\text {flow }}\left(\dot{u}_{\mathrm{r}} \sin \left(-\chi_{\mathrm{r}}+\psi_{\text {flow }}\right)\right)}{2 V_{\text {flow }} u_{\mathrm{r}} \cos \left(-\chi_{\mathrm{r}}+\psi_{\text {flow }}\right)+u_{\mathrm{r}}^{2}+V_{\text {flow }}^{2}} \\
& +\frac{\dot{\chi}_{\mathrm{r}} V_{\text {flow }}\left(u_{\mathrm{r}} \cos \left(-\chi_{\mathrm{r}}+\psi_{\text {flow }}\right)+V_{\text {flow }}\right)}{2 V_{\text {flow }} u_{\mathrm{r}} \cos \left(-\chi_{\mathrm{r}}+\psi_{\text {flow }}\right)+u_{\mathrm{r}}^{2}+V_{\text {flow }}^{2}} \\
& -\dot{\chi}_{\mathrm{r}}+a_{\beta_{\mathrm{r}}} \psi_{\mathrm{r}}-\left(1+b_{\beta_{\mathrm{r}}}\right) r_{\mathrm{r}} \\
& -a_{\beta_{\mathrm{r}}} \tan ^{-1}\left(\frac{V_{\text {flow }} \sin \left(-\chi_{\mathrm{r}}+\psi_{\text {flow }}\right)}{u_{\mathrm{r}}+V_{\text {flow }} \cos \left(-\chi_{\mathrm{r}}+\psi_{\text {flow }}\right)}\right)
\end{aligned}
$$

and

$$
\begin{aligned}
b_{u_{\text {biasr }}}= & \dot{u}_{\mathrm{r}_{\text {new }}}-a_{u_{\mathrm{r}}} u_{\mathrm{r}_{\text {new }}}-\tilde{T}_{\mathrm{r}} \\
= & \frac{\dot{u}_{\mathrm{r}}\left(u_{\mathrm{r}}+V_{\text {flow }} \cos \left(-\chi_{\mathrm{r}}+\psi_{\text {flow }}\right)\right)}{\sqrt{2 V_{\text {flow }} u_{\mathrm{r}} \cos \left(-\chi_{\mathrm{r}}+\psi_{\text {flow }}\right)+u_{\mathrm{r}}^{2}+V_{\text {flow }}^{2}}} \\
& +\frac{V_{\text {flow }} u_{\mathrm{r}}\left(\dot{\chi}_{\mathrm{r}}\right) \sin \left(-\chi_{\mathrm{r}}+\psi_{\text {flow }}\right)}{\sqrt{2 V_{\text {flow }} u_{\mathrm{r}} \cos \left(-\chi_{\mathrm{r}}+\psi_{\text {flow }}\right)+u_{\mathrm{r}}^{2}+V_{\text {flow }}^{2}}} \\
& -\dot{u}_{\mathrm{r}}-a_{u_{\mathrm{r}}}\left(\left(u_{\mathrm{r}}+V_{\text {flow }} \cos \left(-\beta-\psi+\psi_{\text {flow }}\right)\right)^{2}\right. \\
& \left.+V_{\text {flow }}^{2} \sin ^{2}\left(-\chi_{\mathrm{r}}+\psi_{\text {flow }}\right)\right)^{\frac{1}{2}}-\tilde{T}_{\mathrm{r}}
\end{aligned}
$$

Incorporating these new model parameters and reference states in (6.46) restores asymptotic stability of the system (6.34-6.38) in the presence of constant fluid flow unaccounted by the generated reference trajectory.

Proposition 6.3.8. The control law (6.66), with a generated reference trajectory not considering flow, globally asymptotically stabilizes the dynamic system (6.34-6.38) about the equilibrium $\boldsymbol{\eta}=\mathbf{0}$ and $\boldsymbol{\xi}=\mathbf{0}$ in the presence of constant flow with magnitude and direction $V_{\text {flow }}$ and $\psi_{\text {flow }}$ provided the reference trajectory's course and speed are modified using (6.72) and (6.71) and the reference model parameters are adjusted using (6.75) and (6.76). $e_{\psi}$ remains bounded and but does not converge to zero provided $\psi_{\mathrm{r}}, \chi_{\mathrm{r}}, \delta s_{\mathrm{r}}, u_{\mathrm{r}}$, and $\delta n_{\mathrm{r}}$ are bounded, $\tilde{T} \neq 0$, and provided $a_{\beta}$ and $b_{\beta}$ are constant and satisfies $-\frac{a_{\beta}}{1+b_{\beta}}<0$. 
Proof: Consider the error dynamics in (6.34-6.38). Choose the radially unbounded candidate Lyapunov function

$$
V_{2}=\frac{1}{2} \eta_{1}^{2}+\frac{1}{2} \eta_{2}^{2}+\frac{1}{2} \eta_{3}^{2}+\frac{1}{2}(\boldsymbol{\xi}-\boldsymbol{\phi}(\boldsymbol{\eta}))^{T} \boldsymbol{H}^{(i)}(\boldsymbol{\xi}-\boldsymbol{\phi}(\boldsymbol{\eta}))>0
$$

Applying the control law (6.66), the time derivative of (6.77) is

$$
\dot{V}_{2}=-\kappa_{3} \eta_{1} \tanh \left(\kappa_{4} \eta_{1}\right)-\kappa_{1} \eta_{3} \tanh \left(\kappa_{1} \eta_{3}\right)-(\boldsymbol{\xi}-\boldsymbol{\phi}(\boldsymbol{\eta}))^{T} \boldsymbol{K} \boldsymbol{H}^{(i)}(\boldsymbol{\xi}-\boldsymbol{\phi}(\boldsymbol{\eta})) \leq 0
$$

Using Lemma 6.3.1 for the $\boldsymbol{\eta}$ subsystem, and recalling that $K$ is positive and diagonal, the equilibrium is globally asymptotically stable by Lyapunov's direct method, provided $e_{\psi}$ is bounded so that the input (6.66) remains well-defined.

Rearranging the $\xi_{1}$ transform in (6.33) gives

$$
\dot{e}_{\psi}=\frac{a_{\beta}}{b_{\beta}+1} e_{\psi}+\frac{1}{b_{\beta}+1} \xi_{1}+\Delta a_{\beta} \chi_{\mathrm{r}}-\Delta a_{\beta} \psi_{\mathrm{r}}+\Delta b_{\beta} \dot{\psi}_{\mathrm{r}}+\Delta b_{\beta_{\text {bias }}}
$$

Since $\frac{a_{\beta}}{1+b_{\beta}}<0$, the system (6.78) is exponentially stable. Since $\xi_{1}$ remains bounded while the input (6.66) is well-defined and the " $\Delta$ " terms are finite, $e_{\psi}$ (and $\dot{e}_{\psi}$ ) remains bounded; see Lemma 4.6 in [Khalil, 2002]. Note that as $\xi_{1}$ converges to zero, $e_{\psi}$ and $\dot{e}_{\psi}$ remain bounded but do not converge to zero due to parameter discrepancies between the model used to generate trajectories and the models used to estimate actual vessel dynamics.

\subsection{Control Results}

The control laws developed in this section are mostly tested in the field, resulting in good tracking results, primarily for the backstepping control law. First, forward tracking control laws are tested using steady speed, followed by aggressive variable speed, reference trajectories. The backstepping control law outperforms the PD cascade control law prompting the decision to only implement the backstepping sternward motion control law, which shows good tracking performance in the presence of significant actuator limitations. Finally, due to the lack of independent flow measurements and appropriate field flow conditions, a simulation 
of the flow augmented backstepping trajectory tracker is shown in lieu of field implemented results.

\subsubsection{Forward Motion Experimental Results}

Due to the hierarchal nature of the PD trajectory tracking controller, lower level controllers are tested first. Incrementally higher level controllers are tested until finally the trajectory tracking controllers are tested. The performance of the rudder and propeller speed control are first tested. The trajectory tracking control algorithms defined in Sections 6.2, 6.3 and 6.3.1 assume rudder angle and propeller speed are directly controlled. In reality, a motor controller actuates hydraulics that drive the rudder and a throttle actuator sets the throttle position. A PID loop implemented on the motor controller controls rudder motion. A PID controller, coupled with a nonlinear steady-state gain mapping, controls propeller speed. Figures 6.4(a) and 6.4(b) show rudder and propeller speed performance respectively.

Heading control is tested for forward motion, with PD parameters tuned to accommodate rate limiting in the actuators. Figure 6.5(a) shows the boat tracking to a northern heading after facing south. The ratio between proportional and derivative gain varies for different traces, illustrating the effects of derivative control on a system with rate limited inputs. Too much derivative gain causes the system to oscillate on its way to a zero heading while too little derivative gain causes the system to overshoot considerably. The ideal ratio between proportional and derivative control yields no oscillatory behavior or overshoot. This ratio is a function of the rate limit and the maximum achievable turn rate. The rate limit stays constant through the operating envelope, but the maximum turn rate varies, requiring use of gain scheduling with speed.

Wrapping the speed controller around the propeller speed control results in good speed tracking. Figure 6.5(b) shows the boat tracking desired speed by sending propeller speed commands to the propeller speed controller. The speed tracking ability demonstrated in Figure 6.5(b) requires a feedforward propeller speed command that corresponds to the desired forward speed. This feedforward propeller speed command

is replaced by the open-loop time history of commanded propeller speed when the forward speed controller is incorporated into the PD cascade described in Section 6.2.

Figure 6.6 shows the performance of the trajectory tracking controllers with a reference trajectory that has 


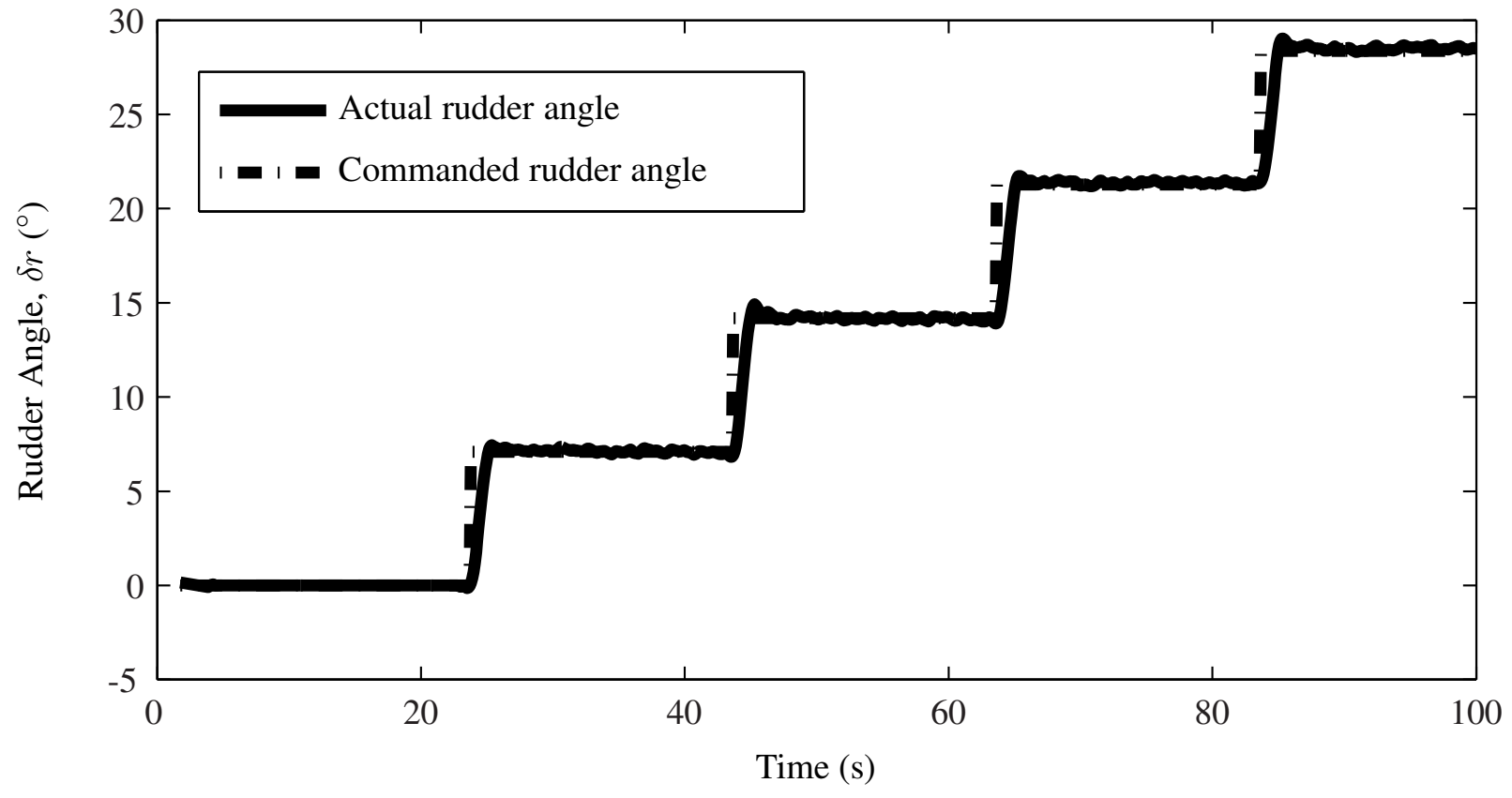

(a) The Ribcraft USV tracking to commanded rudder

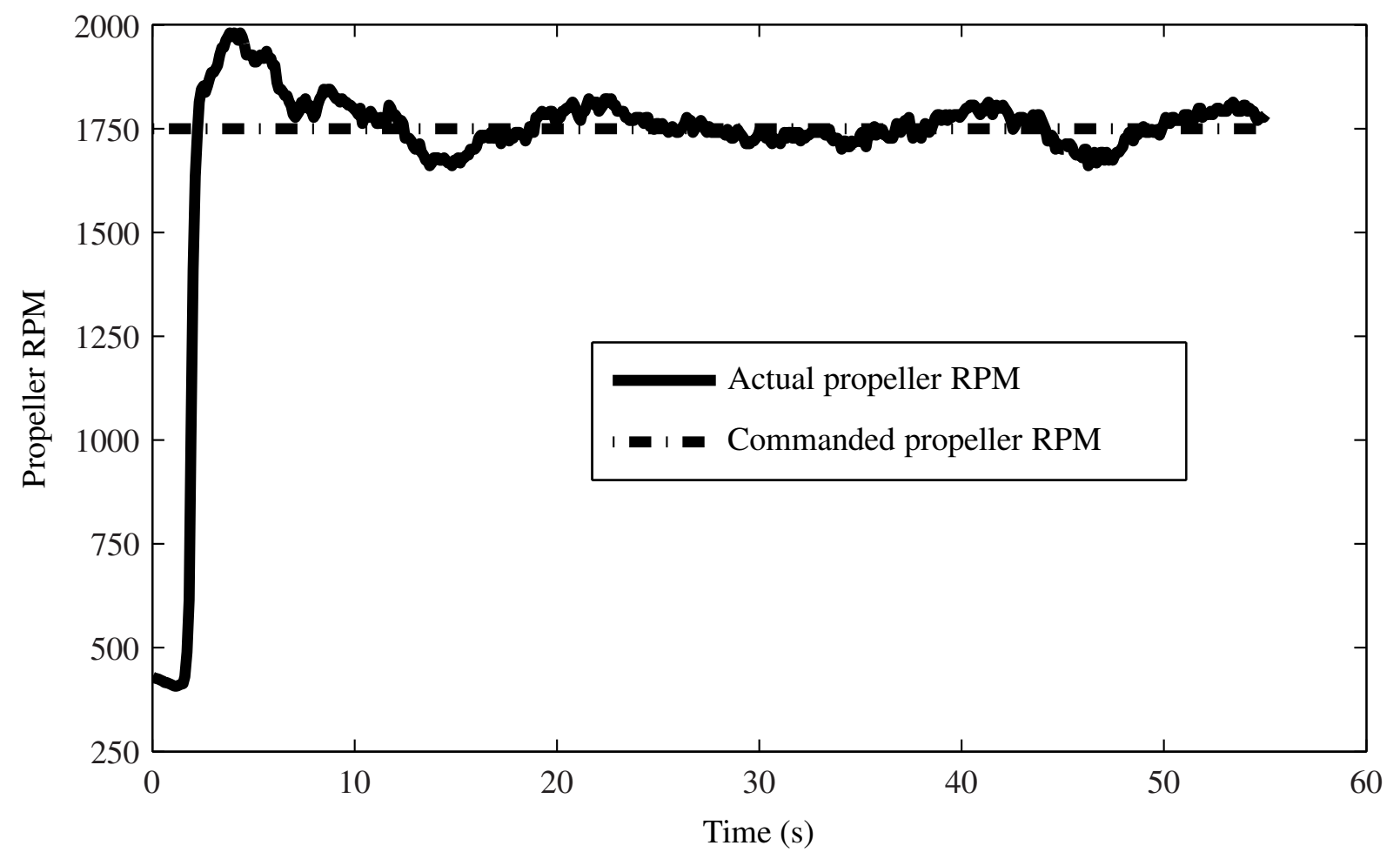

(b) The Ribcraft USV tracking to commanded propeller speed

Figure 6.4: Actuator control performance 


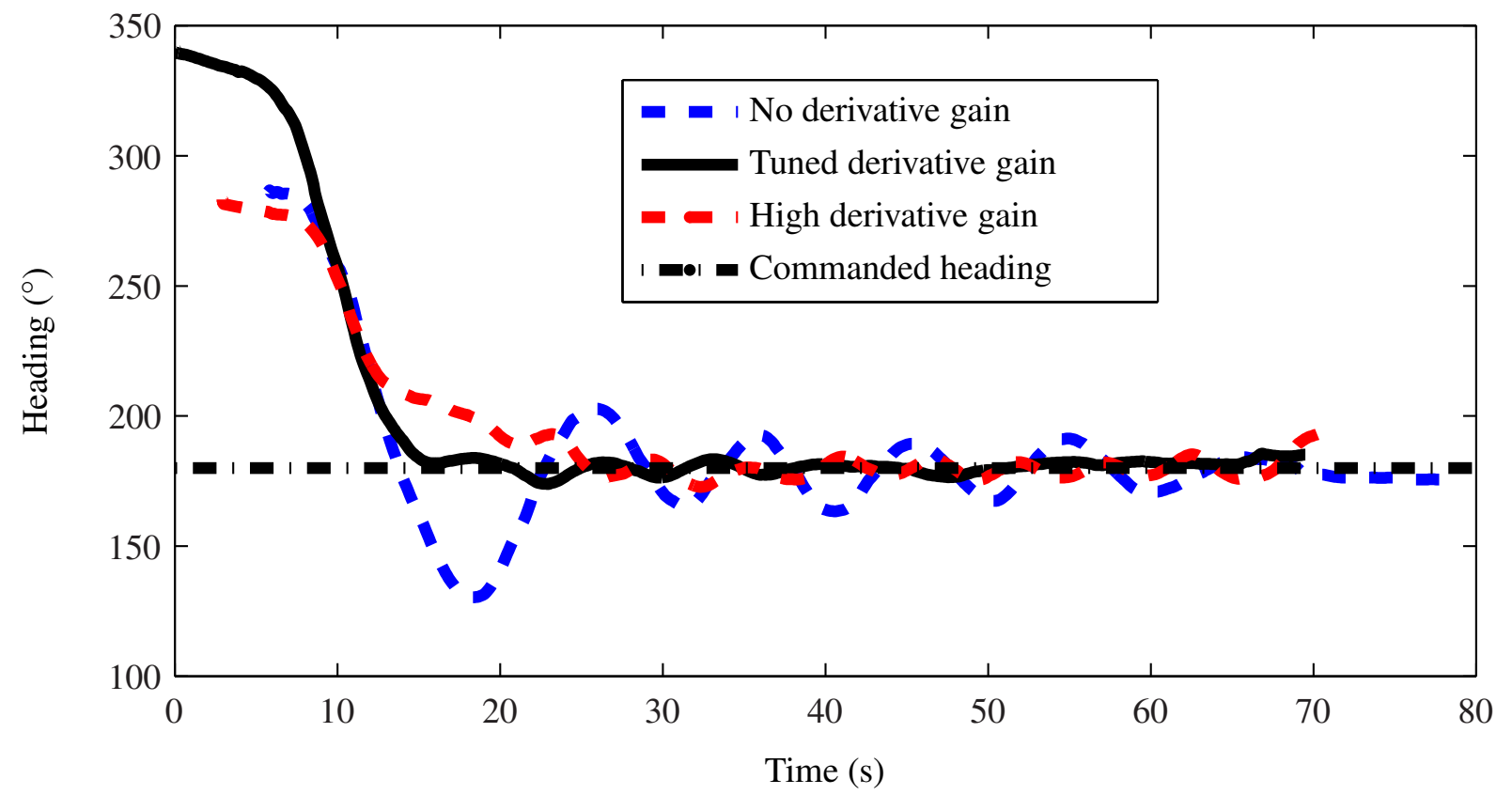

(a) The Ribcraft USV tracking to commanded heading for different ratios of proportional and derivative gains

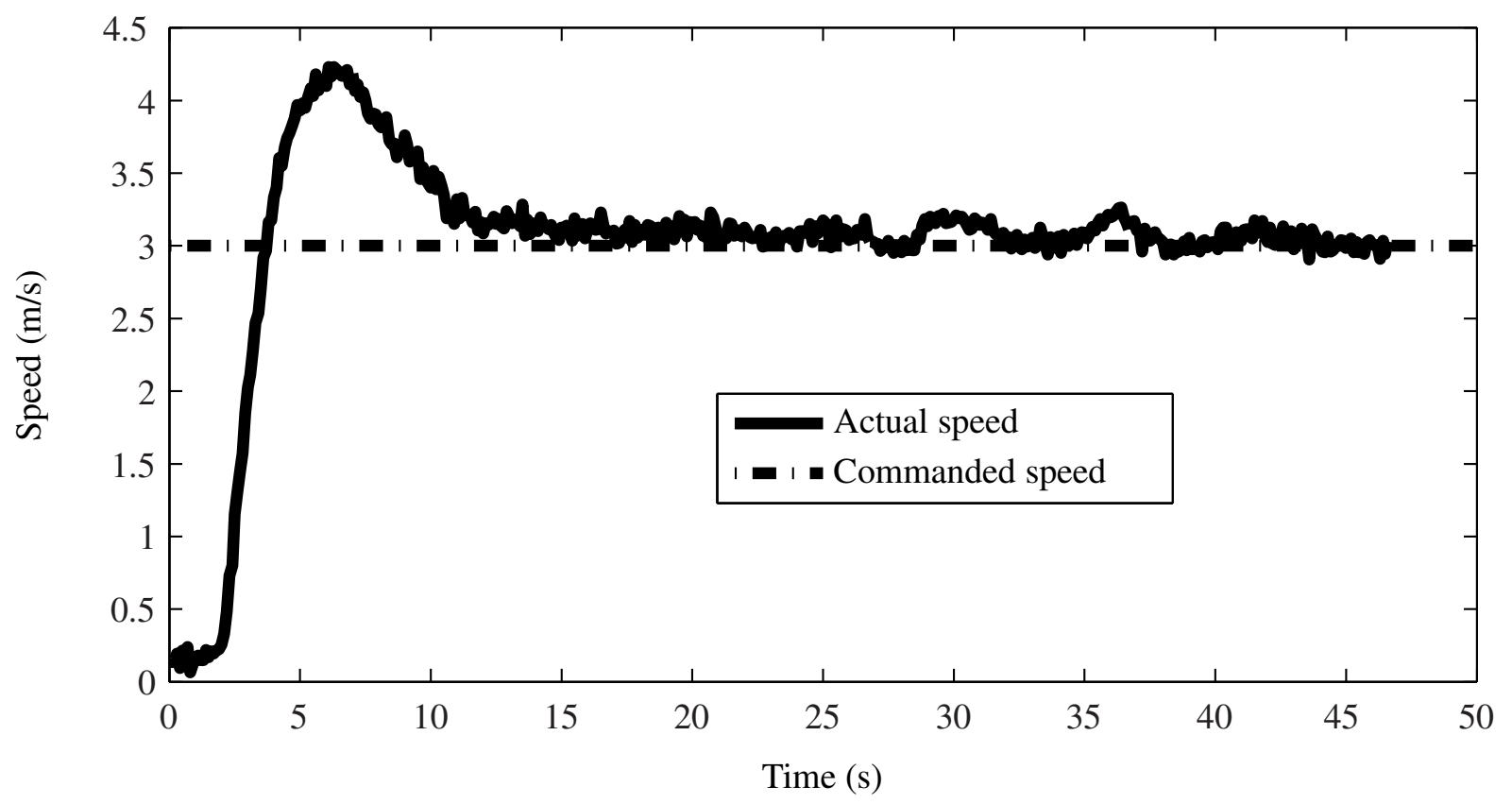

(b) The Ribcraft USV tracking to commanded speed

Figure 6.5: Heading and speed tracking performance 
constant propeller speed and a sinusoid in rudder angle. (All trajectories begin at the origin.) Circles and velocity vectors denote position and velocity at ten second intervals. The backstepping trajectory tracking control law, implemented without considering Lyapunov function discontinuities, immediately converges to the reference trajectory and stays within a few meters of the desired position. The PD cascade trajectory tracking control law results in slightly poorer tracking performance than the backstepping control law.

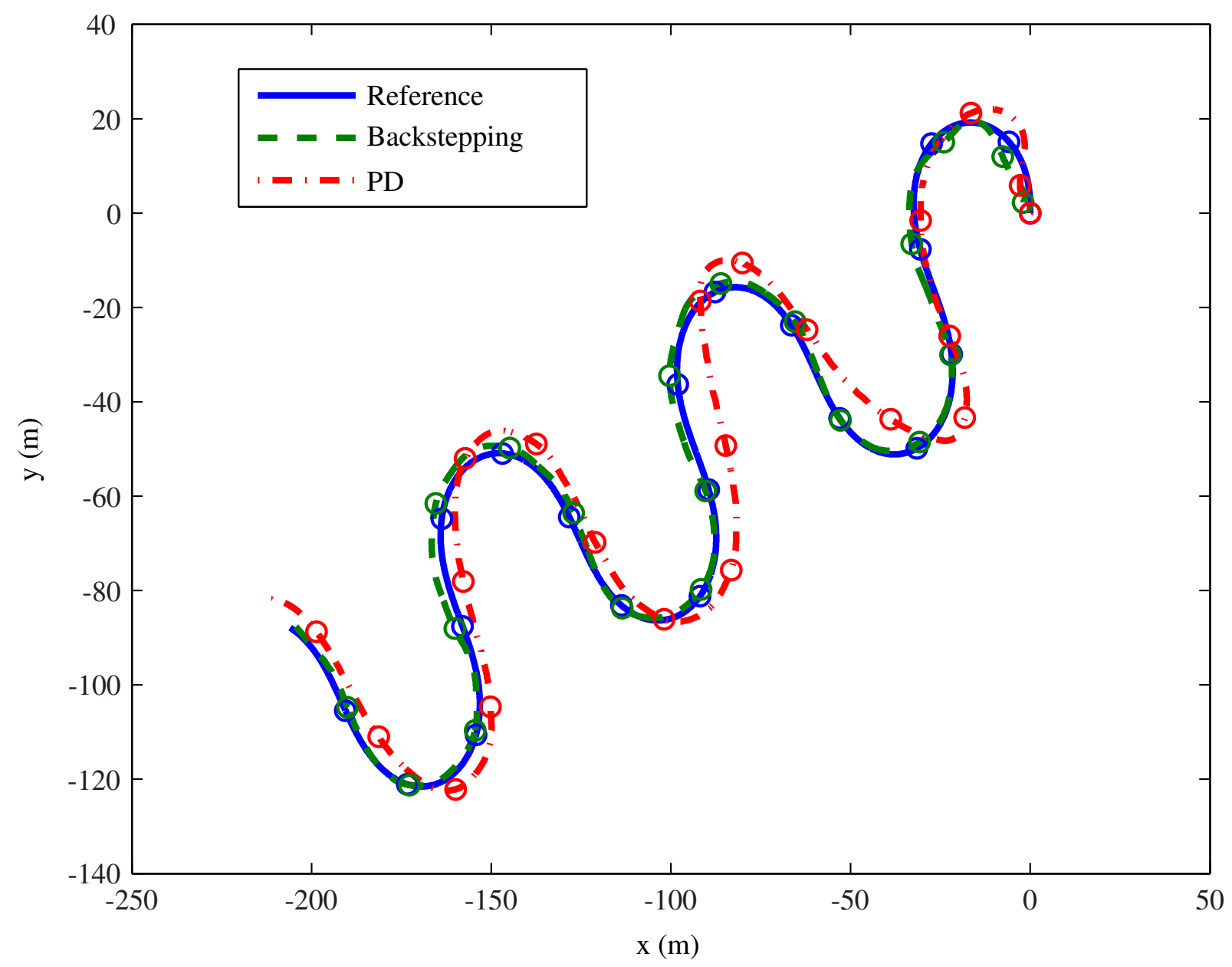

Figure 6.6: Trajectory tracking control for constant propeller speed and a sinusoid in rudder

Figure 6.7 shows the performance of the trajectory tracking controllers with a reference trajectory that has a sinusoid in propeller speed coupled with a sinusoid (of higher frequency) in rudder angle. This reference 
trajectory contains aggressive maneuvering including taking sharp turns during hard deceleration and acceleration. Since the reference speed varies, parameter switching in the control laws is tested. Furthermore, such an aggressive reference trajectory tests the controllers' effectiveness in the presence of unmodeled dynamics including interaction with wake dynamics that are noticeable during simultaneous deceleration and turning. Like the previous trajectory plot, circles and velocity vectors denote position and velocity at ten second intervals. The backstepping trajectory tracking control law forces convergence to the reference trajectory and the vessel stays within a few meters of the desired position, though oscillations are noticeable when wake dynamics become prevalent. The PD cascaded trajectory tracking control results in qualitatively correct behavior, but the vessel's trajectory fails to converge to the reference trajectory.

The backstepping control law clearly outperforms the PD cascaded control law for the field tests presented in Figures 6.6 and 6.7. For steady speed reference trajectories the PD cascaded control law oscillates about the reference trajectory while the backstepping control law converges quickly. For variable speed reference trajectories the PD cascaded control law's performance degrades considerably while the backstepping control law converges to the reference trajectory.

\subsubsection{Sternward Motion Experimental Results}

Figure 6.8 shows the performance of the trajectory tracking controller with a reference trajectory that has constant propeller speed and a sinusoid in thrust angle. Circles and velocity vectors denote position and velocity at twenty second intervals. The backstepping trajectory tracking control law immediately converges to the reference trajectory and stays within a few meters of the desired position indicated by the close correlation of the reference and backstepping position circles and velocity vectors. At times where the vehicle switches to a different turn rate region the vessel briefly diverges from the desired position due to short times of unavoidable actuator saturation. Figure 6.9 shows the propeller speed and rudder position during the trajectory run in Figure 6.8. Note that the rudder is consistently rate limited and/or saturated throughout the run, yet the control law with the tuned control gains in Table 6.4 tracks the trajectory well. 


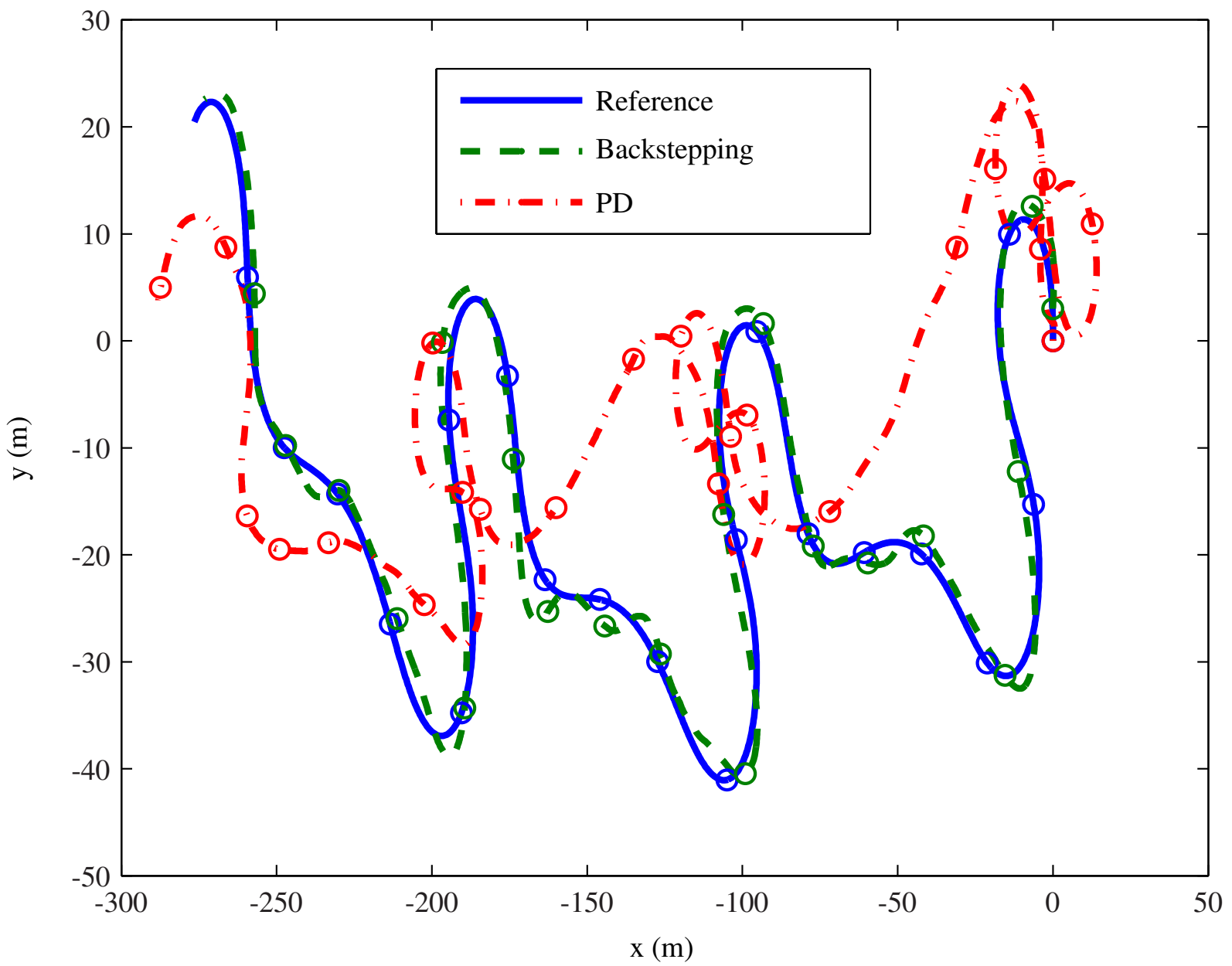

Figure 6.7: Trajectory tracking control for sinusoids (of differing frequency) in propeller speed and rudder

\subsubsection{Control in Flow Simulation Results}

Figure 6.10 shows the performance of the trajectory tracking controllers in a constant North to South flow of $1 \mathrm{~m} / \mathrm{s}$ using a reference trajectory generated with constant propeller speed, a sinusoid in thrust angle, and the $1 \mathrm{~m} / \mathrm{s}$ North to South constant flow. Circles and velocity vectors denote position and velocity at ten second intervals. The backstepping trajectory tracking control law results in immediate convergence toward the reference trajectory; notice the close correlation of the actual and reference positions and velocity vectors. 


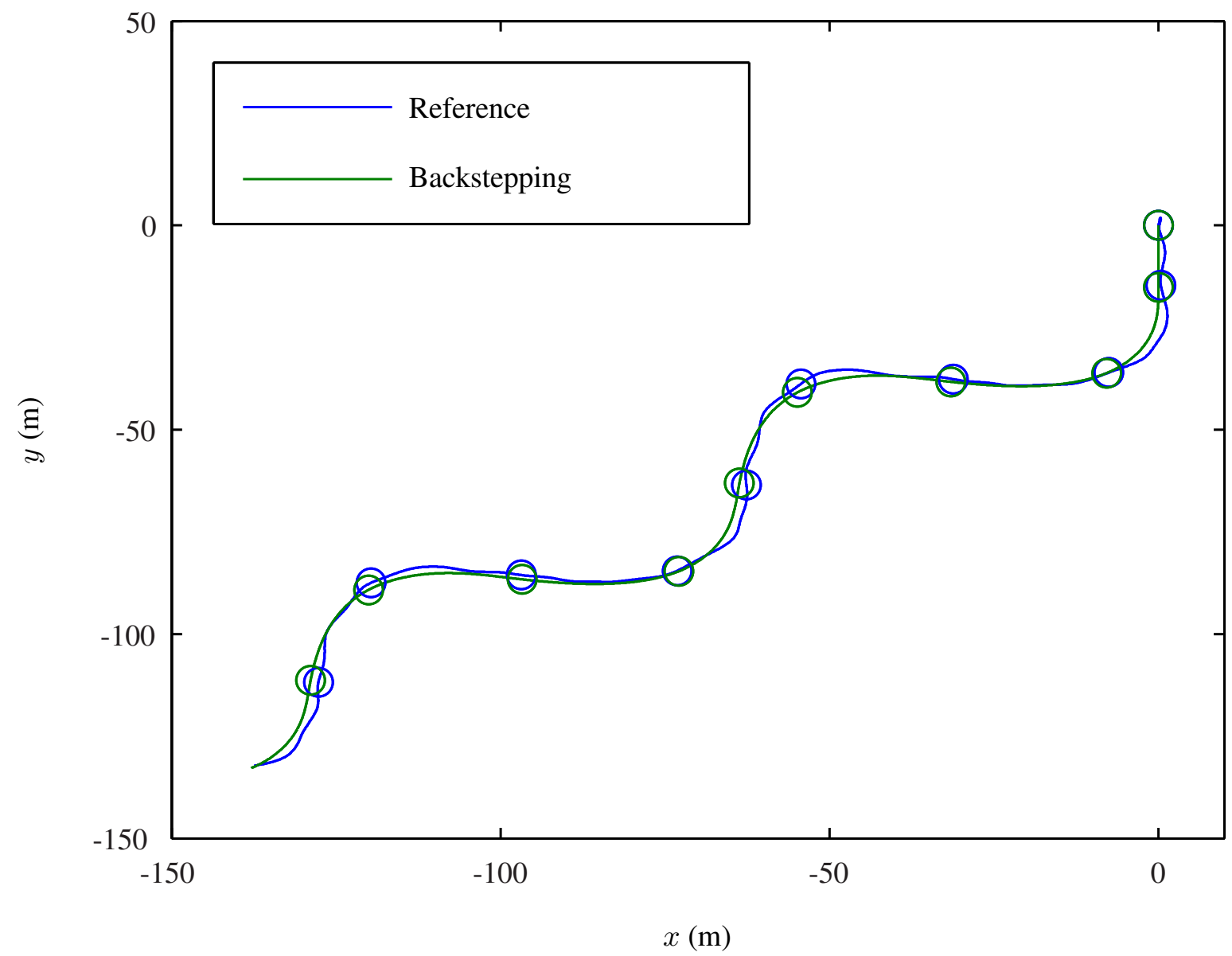

Figure 6.8: Trajectory tracking control for constant propeller speed and a sinusoid in thrust angle in sternward motion

The PD cascade trajectory tracking control law, which modifies the reference trajectory heading to steer the vessel toward the path, results in sloppy performance similar to the experimental results. Note that the convergence properties in flow and without flow are identical if the reference trajectory is convected with the same flow as the vessel.

Figure 6.11 shows the performance of the trajectory tracking controllers in a constant North to South flow of $1 \mathrm{~m} / \mathrm{s}$ using a reference trajectory generated with a constant propeller speed and a sinusoid in thrust 


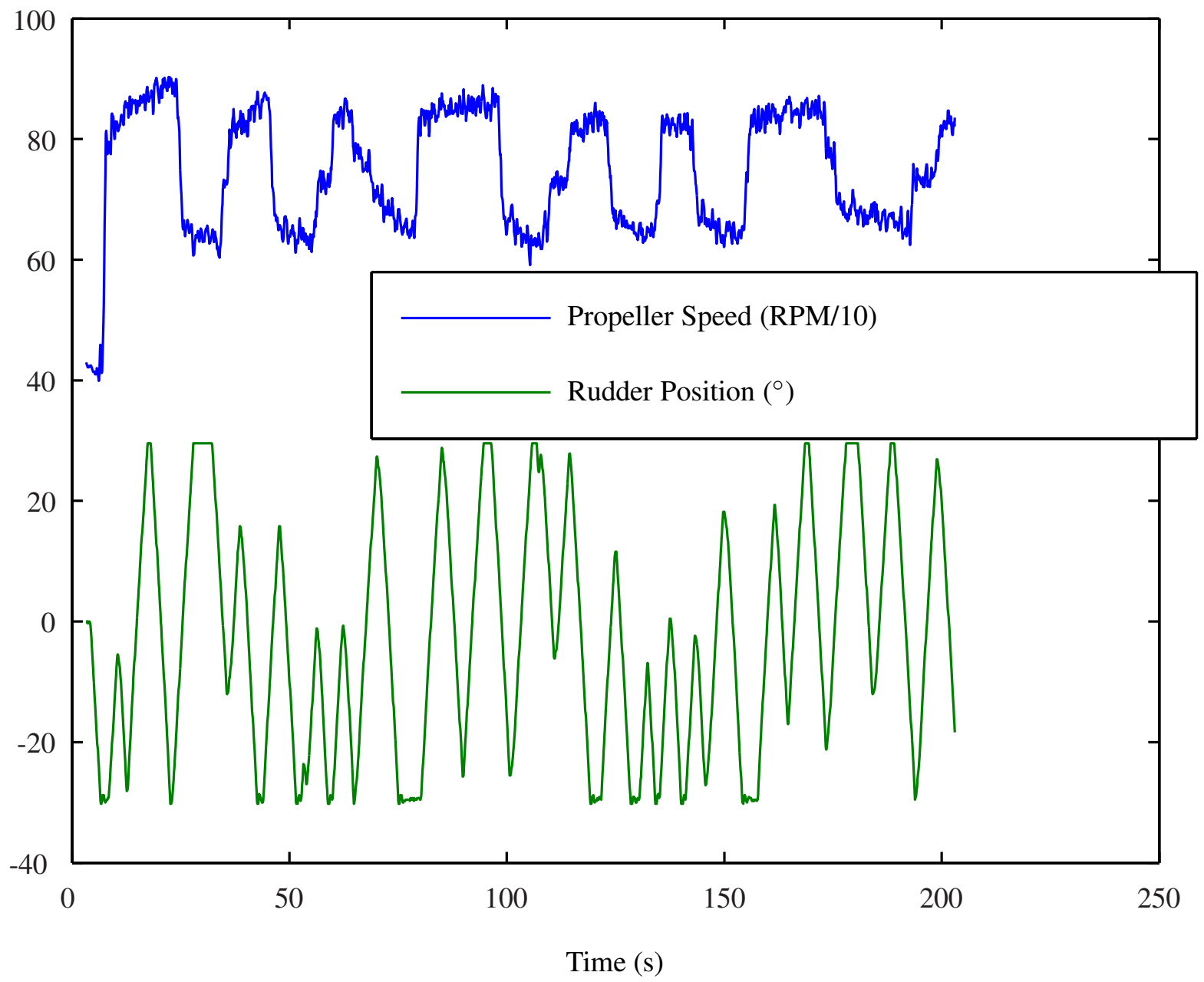

Figure 6.9: Actual actuator inputs of propeller speed and rudder position used in the trajectory tracking control run in Figure 6.8

angle and without knowledge of the $1 \mathrm{~m} / \mathrm{s}$ North to South flow. Like Figure 6.10, Figure 6.11 has circles and velocity vectors denoting position and velocity at ten second intervals. The backstepping trajectory tracking control law with flow compensation results in immediate convergence, as in the previous case shown in Figure 6.10. The backstepping trajectory tracking control law without flow compensation results in convergence with a roughly $5 \mathrm{~m}$ south steady state error from the desired path. The PD cascaded trajectory 


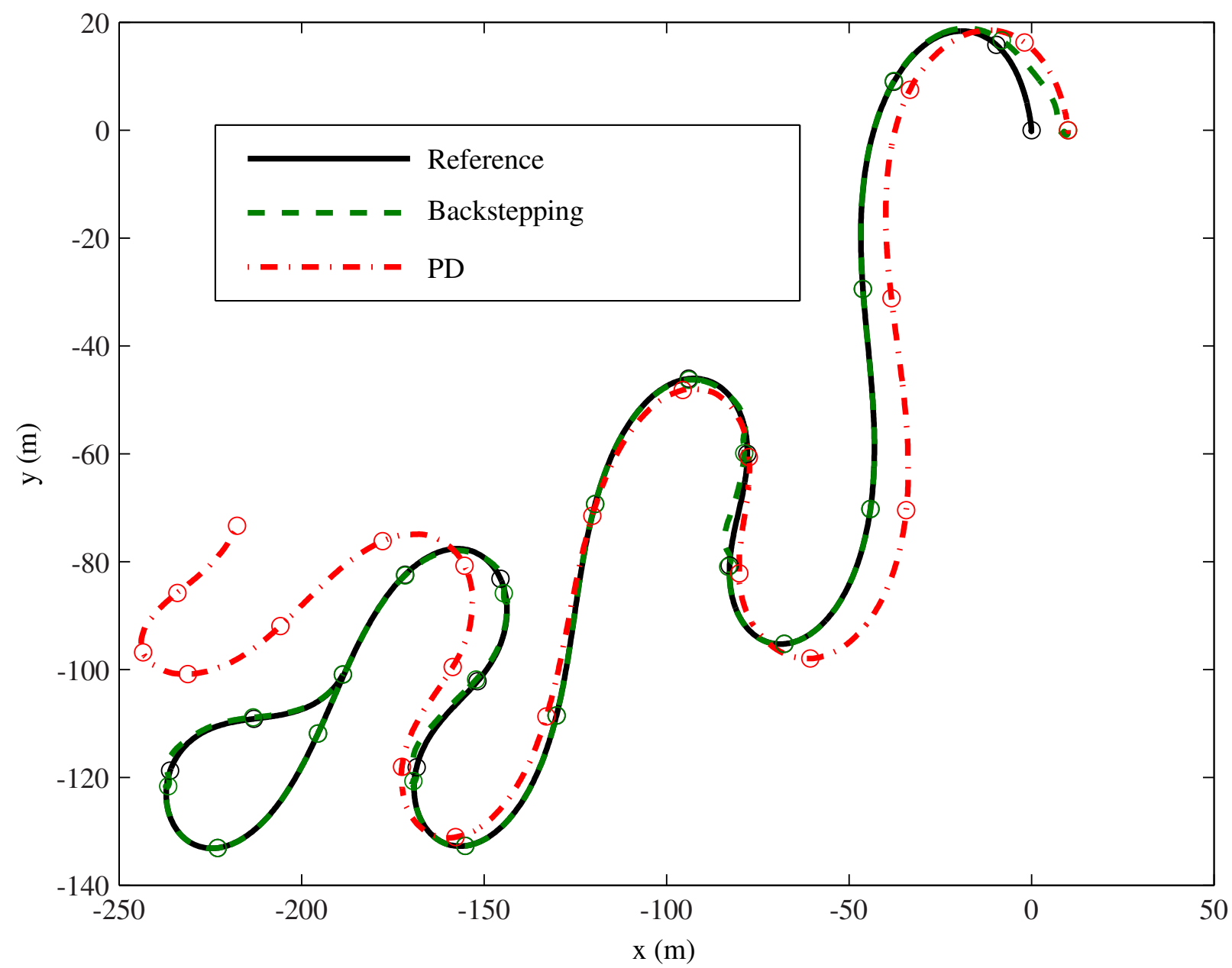

Figure 6.10: Trajectory tracking control for constant propeller speed and a sinusoid in thrust angle with the generated reference trajectory and actual vessel both being convected with flow

tracking control results in extremely poor performance.

The backstepping control law clearly outperforms the PD cascaded control law in the simulations shown in Figures 6.10 and 6.11. In cases where the generated flow trajectory shares the same fluid flow as the actual vessel, stability properties are unchanged. The backstepping control law remains quite effective when a known flow is directly compensated for. Simulations underscore that the backstepping control law is also effective when there is a mismatch between the true and assumed flow, while the PD cascade control law 


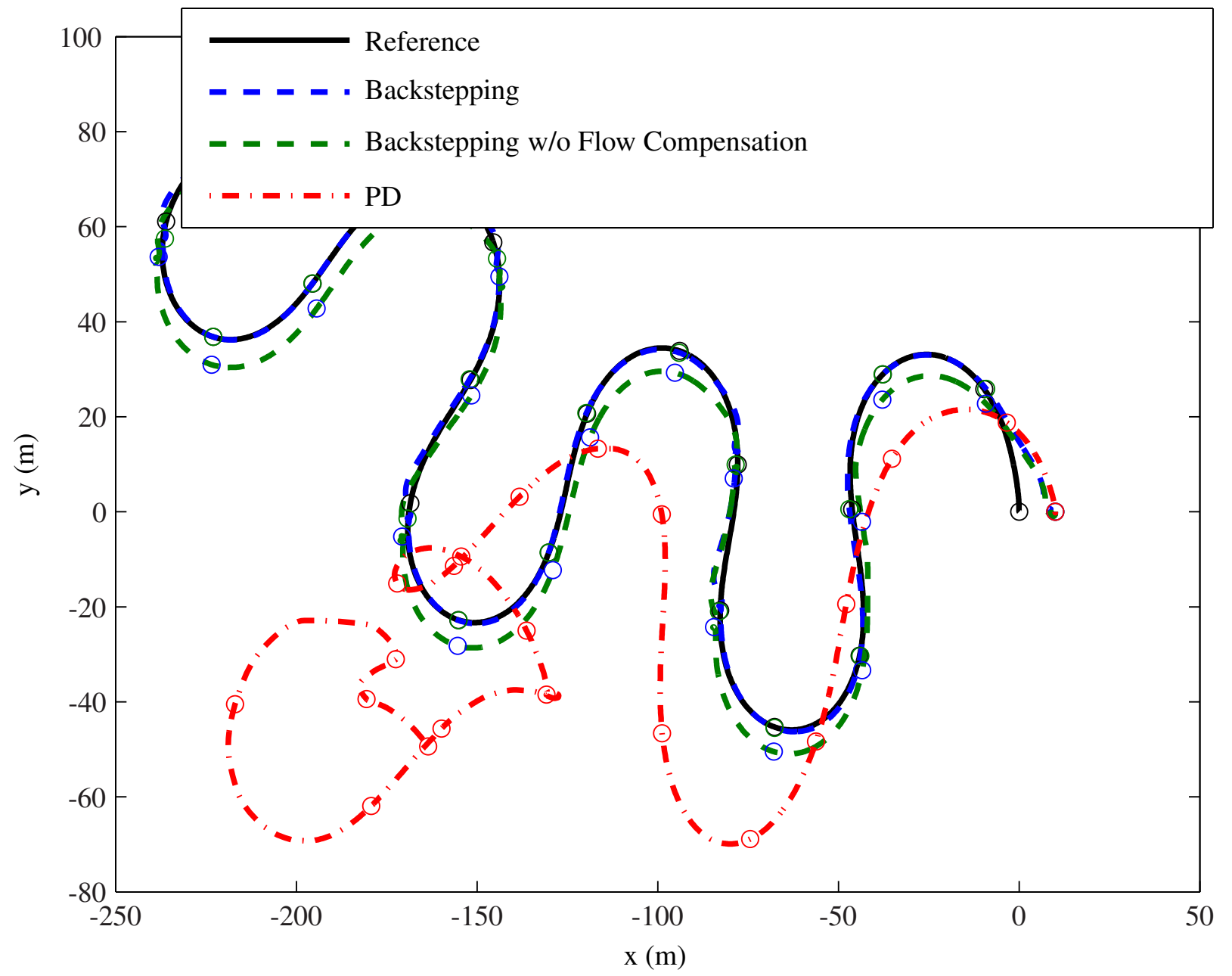

Figure 6.11: Trajectory tracking control for constant propeller speed and a sinusoid in thrust angle with the reference trajectory generated assuming no flow with the actual vessel in flow

performs very poorly. 


\section{CHAPTER}

\section{CONCLUSIONS}

By combining experimental parameter identification for simple motion models with a model-based trajectory tracking algorithm, a new field tested maneuvering method causes the planing Ribcraft USV to converge to aggressive reference trajectories. The simple motion models include a globally valid thruster model and a set of locally valid linear steering and speed models that together sufficiently capture planing vessel dynamics. A trajectory tracking control law is developed using the simple models, yielding global asymptotic stability about a trajectory for a planing vessel.

A parameter identification method is developed to identify all transient dynamics. First, thruster dynamics, which are valid through the entire forward motion regime, are identified by using a unique set of field experiments that accentuate only dynamics dependent on thrust. Second, linear equations are fit to steady state steering behavior for a range of forward speeds, encompassing the displacement, semi-displacement, and planing regions. Exciting the vessel dynamics by providing closed-loop commands in heading and 
speed gives rich enough data sets to identify transient forward motion.

The Ribcraft USV and other planing vessels are streamlined for forward motion. Performance in sternward motion is not as good. Specifically designed closed-loop turn rate field experiments separately excite the stable left and right turn dynamics as well as the unstable zero turn rate equilibrium. These complex dynamics are modeled with turn rate scheduled linear steering models for each region, which are identified using the previously stated identification procedure in conjunction with the turn rate field experiments. Thrust and speed parameter identification mirrors the methods used for forward identification, but only a single thrust parameter and single set of speed parameters are found due to prohibitive speed constraints.

A PD cascade trajectory tracking controller and a backstepping trajectory tracking controller are developed to force the Ribcraft USV to track feasible trajectories generated using the identified set of steering and speed models. The standard PD cascade trajectory tracking controller, which does not intrinsically account for vessel dynamics, is implemented as a baseline that the backstepping control law is compared against. The PD controller first uses position error to augment commanded reference speed and heading, which are then tracked using simple lower level controllers.

The backstepping trajectory tracking controller, like the PD controller, causes the Ribcraft USV to converge to a time-varying reference state history. First, the problem is formulated to account for a single set of steering and speed parameters. Switching between additional parameter sets is accomplished by understanding discrepancies in parameters between the reference and actual vessel and by preventing potential discontinuities in the Lyapunov function. Field experiments show superior trajectory tracking of the backstepping control law over the PD cascade control law for steady forward speed and aggressive variable forward speed reference trajectories. The backstepping trajectory tracking control law for forward motion assumes that actual and estimated vessel parameters match once all errors have been attenuated, which is not true for sternward motion.

Discrepancies between reference and actual sternward model parameters persist for all time since a single stable model is used to generate a sternward reference trajectory while two stable models and one unstable model describe the actual Ribcraft USV sternward steering motion. The backstepping control law remains unchanged between forward and sternward motion cases; however, in sternward motion the heading error re- 
mains bounded but does not converge to zero. This boundedness is acceptable since all other error terms are attenuated. Field experiments demonstrate that the backstepping control law overcomes bistable dynamics and actuator limitations to track to a reference trajectory.

The trajectory tracking control laws intrinsically account for fluid flow if the flow is constant, the reference trajectory is generated in the flow, and flow relative velocity is measured. Both the reference and actual vehicle states are simply convected along the flow. However, by augmenting reference trajectories generated without the knowledge of the flow condition, lost stability is reclaimed. Additionally, the bias terms in the reference steering and speed models must be adjusted to rectify the disconnect between the original and augmenting reference trajectory. With discrepancies again appearing in terms between the reference and actual models the heading error remains bounded but does not converge to zero. Due to the absence of flow measuring devices and appropriate environments with fluid flow, simulations instead of field experiments confirm the control law's stability. 


\section{BIBLIOGRAPHY}

[Abkowitz, 1964] Abkowitz, M. A. (1964). Lectures on ship hydrodynamics - steering and maneuverability. Technical report, Hydro- and Aerodynamic's Laboratory, Lyngby, Denmark.

[Aircardi et al., 2001] Aircardi, M., Casalino, G., Indiveri, G., Aguiar, A., Encarnacao, P., and Pascoal, A. (2001). A planar path following controller for underactuated marine vehicles. In 9th IEEE Mediterranean Conference on Control and Automation, Dubrovnik, Croatia.

[Almeida et al., 2009] Almeida, C., Franco, T., Ferreira, H., Martins, A., Santos, R., Almeida, J., Carvalho, J., and Silva, E. (2009). Radar based collision detection developments on USV ROAZ II. In OCEANS 2009 - EUROPE, pages 1-6, Bremen, Germany.

[Alves et al., 2006] Alves, J., Oliveira, P., Oliveira, R., Pascoal, A., Rufino, M., Sebastião, L., and Silvestre, C. (2006). Vehicle and mission control of the DELFIM autonomous surface craft. In In Proc. 14th Mediterranean Conference on Control and Automation, pages 1-6, Sardinia, Italy.

[Amerongen and Cappelle, 1981] Amerongen, J. V. and Cappelle, J. C. V. (1981). Mathematical modelling for rudder roll stabilization. In 6th International Ship Control Systems Symposium, Ottawa, Canada. 
[Armaoglu et al., 2006] Armaoglu, E., Ayaz, Z., Vassalos, D., and T, K. (2006). On manoeuvrability of semi-displacement craft in astern seas. In In Proceedings of the 9th International Conference on Stability and of Ships and Ocean Vehicles, Rio de Janeiro, Brazil.

[Åström and Källström, 1976] Åström, K. J. and Källström, C. G. (1976). Identification of ship steering dynamics. Automatica, 12:9-22.

[Ayaz et al., 2006] Ayaz, Z., Vassalos, D., and Spyrou, K. J. (2006). Manoeuvring behaviour of ships in extreme astern seas. Ocean Engineering, 33:2381-2434.

[Bayard, 1994a] Bayard, D. S. (1994a). An algorithm for state-space frequency domain identification without windowing distortions. IEEE Transactions on Automatic Control, 39(9):1880-1885.

[Bayard, 1994b] Bayard, D. S. (1994b). High-order multivariable transfer function curve fitting: Algorithms, sparse matrix methods and experimental results. Automatica, 30:1439-1444.

[Beltrami, 1998] Beltrami, E. (1998). Mathematics for Dynamic Modeling. Academic Press, New York, NY, second edition.

[Bibuli et al., 2008] Bibuli, M., Bruzzone, G., Caccia, M., Indiveri, G., and Zizzari, A. (2008). Line following guidance control: Application to the Charlie unmanned surface vehicle. In IEEE/RSJ International Conference on Intelligent Robots and Systems, 2008. IROS 2008., pages 3641-3646.

[Bibuli et al., 2009] Bibuli, M., Bruzzone, G., Caccia, M., and Lapierre, L. (2009). Path-following algorithms and experiments for an unmanned surface vehicle. Journal of Field Robotics, 26(8):669-688.

[Bibuli et al., 2007] Bibuli, M., Caccia, M., and Lapierre, L. (2007). Path-following algorithms and experiments for an autonomous surface vehicle. In In Proceedings of IFAC Conference on Control Applications in Marine Systems.

[Blanke, 1981] Blanke, M. (1981). Ship Propulsion Losses Related to Automated Steering and Prime Mover Control. PhD thesis, The Technical University of Denmark, Lyngby, Denmark.

[Breivik, 2010] Breivik, M. (2010). Topics in Guided Motion Control of Marine Vehicles. PhD thesis, Norwegian University of Science and Technology.

[Breivik and Fossen, 2004] Breivik, M. and Fossen, T. (2004). Path following for marine surface vessels. In OCEANS '04. MTTS/IEEE TECHNO-OCEAN '04, volume 4, pages 2282-2289. 
[Breivik et al., 2008] Breivik, M., Hovstein, V. E., and Fossen, T. I. (2008). Straight-line target tracking for unmanned surface vehicles. Modeling, Identification, and Control, 29(4):131-149.

[Brinati, 1973] Brinati, H. L. (1973). System identification applied to maneuvering trials. Master's thesis, Massachusetts Institute of Technology.

[Bruzzone et al., 2011] Bruzzone, G., Bruzzone, G., Bibuli, M., and Caccia, M. (2011). Autonomous mine hunting mission for the Charlie USV. In OCEANS 2011, pages 1-6, Santander, Spain.

[Caccia et al., 2008a] Caccia, M., Bibuli, M., Bono, R., Bruzzone, G., Bruzzone, G., and Spirandelli, E. (2008a). Unmanned marine vehicles at CNR-ISSIA. In In Proceedings of IFAC Conference on Control Applications in Marine Systems, pages 3070-3075, Seoul, Korea.

[Caccia et al., 2009] Caccia, M., Bibuli, M., Bono, R., Bruzzone, G., and Spirandelli, E. (2009). Aluminum hull usv for coastal water and seafloor monitoring. In OCEANS 2009 - EUROPE, pages 1-5.

[Caccia et al., 2007] Caccia, M., Bibuli, M., R., B., Bruzzone, G., and E., S. (2007). Unmanned surface vehicle for coastal and protected waters applications: the Charlie project. Marine Technology Society Journal, 41(2):62-71.

[Caccia et al., 2006] Caccia, M., Bruzzone, G., and Bono, R. (2006). Modelling and identification of the Charlie2005 ASC. In 14th Mediterranean Conference on Control and Automation, 2006, pages 1-6.

[Caccia et al., 2008b] Caccia, M., Bruzzone, G., and Bono, R. (2008b). A practical approach to modeling and identification of small autonomous surface craft. Oceanic Engineering, IEEE Journal of, 33(2):133145.

[Chen and Billings, 1992] Chen, S. and Billings, S. A. (1992). Neural networks for nonlinear dynamic modelling and identification. International Journal of Control, 56(2):319-346.

[Chen et al., 1990] Chen, S., Billings, S. A., and Grant, P. M. (1990). Nonlinear system identification using neural networks. International Journal of Control, 51(6):1191-1214.

[Christensen and Blanke, 1986] Christensen, A. and Blanke, M. (1986). A linearized state-space model in steering and roll of a high-speed container ship. Technical Report 86-D-574, Technical University of Denmark, Denmark.

[Curcio et al., 2005] Curcio, J., Leonard, J., and Patrikalakis, A. (2005). SCOUT - a low cost autonomous 
surface platform for research in cooperative autonomy. In Proceedings of MTS/IEEE OCEANS, pages 725-729, Washington, D.C.

[Do et al., 2002a] Do, K., Jiang, Z., and Pan, J. (2002a). Underactuated ship global tracking under relaxed conditions. IEEE Transactions on Automatic Control, 47(9):1529-1536.

[Do et al., 2002b] Do, K. D., Jiang, Z. P., and Pan, J. (2002b). Universal controllers for stabilization and tracking of underactuated ships. Systems and Control Letters, 47:299-317.

[DuVal, 1981] DuVal, R. W. (1981). The use of frequency method in rotorcraft system identification. In AIAA/SETP/SFTE/SAE/ITEA/IEEE 1st Flight Testing Conference, Las Vegas, Nevada.

[Faltinsen, 2005] Faltinsen, O. M. (2005). Hydrodynamics of High-Speed Marine Vehicles. Cambridge University Press.

[Fierro and Lewis, 1995] Fierro, R. and Lewis, F. L. (1995). Control of a nonholonomic mobile robot: Backstepping kinematics into dynamics. In Proceedings of the $34 \mathrm{rd}$ Conference on Decision and Control, New Orleans, LA.

[Fossen, 1991] Fossen, T. I. (1991). Nonlinear Modelling and Control of Underwater Vehicles. PhD thesis, The Norwegian Institute of Technology, Trondheim, Norway.

[Fossen, 1995] Fossen, T. I. (1995). Guidance and Control of Ocean Vehicles. John Wiley and Sons.

[Fossen et al., 1996] Fossen, T. I., Sagatun, S. I., and Sørensen, A. J. (1996). Identification of dynamically positioned ships. Modeling, Identification, and Control, 17(2):153-165.

[Gadre et al., 2012a] Gadre, A. S., Du, S., and Stilwell, D. J. (2012a). A topological map based approach to long range operation of an unmanned surface vehicle. In American Control Conference (ACC), 2012.

[Gadre et al., 2009] Gadre, A. S., Kragelund, S., Masek, T., Stilwell, D., Woolsey, C., and Horner, D. (2009). Subsurface and surface sensing for autonomous navigation in a riverine environment. In In Proceedings to AUVSI's Unmanned Systems, Washington, D. C.

[Gadre et al., 2012b] Gadre, A. S., Sonnenburg, C., Du., S., Stilwell, D. J., and Woolsey, C. A. (2012b). Guidance and control of an unmanned surface vehicle exhibiting sternward motion. In in Proceedings of MTS/IEEE Oceans, Hampton Roads, VA.

[Godhavn, 1996] Godhavn, J.-M. (1996). Nonlinear tracking of underactuated surface vessels. In Proceed- 
ings of the 35th Conference on Decision and Control, pages 975-980, Kobe, Japan.

[Grewal and Glover, 1976] Grewal, M. S. and Glover, K. (1976). Identifiability of linear and nonlinear dynamical systems. IEEE Transactions on Automatic Control, 21(6):833-837.

[Gu and Khargonekar, 1992] Gu, G. and Khargonekar, P. (1992). Linear and nonlinear algorithms for identification in $\mathcal{H}_{\infty}$ with error bounds. Automatic Control, IEEE Transactions on, 37(7):953-963.

[Gupta, 1978] Gupta, W. J. N. K. (1978). Instrumental variables algorithm for modal parameter identification in flutter testing. AIAA Journal, 16(8):800-806.

[Hamel and Jategaonkar, 1996] Hamel, P. G. and Jategaonkar, R. V. (1996). Evolution of flight vehicle system identification. Journal of Aircraft, 33(1):9-28.

[Hayes, 1971] Hayes, M. N. (1971). Parametric Identification of Nonlienar Stochastic Systems Applied to Ocean Vehicle Dynamics. PhD thesis, Massachusetts Institute of Technology.

[Helmicki et al., 1991] Helmicki, A., Jacobson, C., and Nett, C. (1991). Control oriented system identification: a worst-case/deterministic approach in $\mathcal{H}_{\infty}$. IEEE Transactions on Automatic Control, 36(10):1163-1176.

[Ikeda et al., 2000] Ikeda, Y., Katayama, T., and Okumura, H. (2000). Characteristics of hydrodynamic derivatives in maneuvering equations for super high-speed planing hulls. In In Proceedings of the 10th International OFFSHORE AND POLAR ENGINEERING CONFERENCE, volume 4, pages 434-444.

[Jacobson and Tadmor, 1993] Jacobson, C. A. and Tadmor, G. (1993). A note on $\mathcal{H}_{\infty}$ system identification with probabilistic apriori information. In American Control Conference, San Francisco, CA.

[Jategaonkar, 2006] Jategaonkar, R. V. (2006). Flight Vehicle System Identification: A Time Domain Methodology. American Institute of Aeronautics and Astronautics, Inc.

[Javanmardi et al., 2008] Javanmardi, M., Seif, M., Jahanbakhsh, E., and Sayyaadi, H. (2008). Trimaran manoeuvring simulation based on three-dimensional viscous free surface flow solver. In In Proceedings of the 6th International Conference on High-Performance Marine Vehicles, Naples, Italy.

[Jiang, 2002] Jiang, Z.-P. (2002). Global tracking control of underactuated ships by Lyapunov's direct method. Automatica, 38:301-309.

[Jiang et al., 2001] Jiang, Z.-P., Lefeber, E., and Nijmeijer, H. (2001). Saturated stabilization and tracking 
of a nonholonomic mobile robot. Systems and Control Letters, 42(5):327-332.

[Jiang and Nijmeijer, 1997] Jiang, Z.-P. and Nijmeijer, H. (1997). Tracking control of mobile robots: A case study in backstepping. Automatica, 33(7):1393-1399.

[Juang, 1994] Juang, J.-N. (1994). Applied System Identification. Prentice Hall, Upper Saddle River, N. J.

[Källström and Åström, 1981] Källström, C. G. and Åström, K. J. (1981). Experience of system identification applied to ship steering. Automatica, 17(1):187-198.

[Kanayama et al., 1990] Kanayama, Y., Kimura, Y., Miyazaki, F., and Noguchi, T. (1990). A stable tracking control method for an autonomous mobile robot. In Robotics and Automation, 1990. Proceedings., 1990 IEEE International Conference on, volume 1, pages 384-389.

[Katayama et al., 2001] Katayama, T., Teshima, A., and Ikeda, Y. (2001). A study on transverse porpoising of a planing craft in drifting motion. 1st report. measurements of motions and mechanism of occurrence. Journal of the Kansai Society of Naval Architects, (236):181-190.

[Katayama et al., 2002] Katayama, T., Teshima, A., and Ikeda, Y. (2002). A study on transverse porpoising of a planing craft in drifting motion. 2nd report. simulation method of transverse porpoising. Journal of the Kansai Society of Naval Architects, 237:149-154.

[Khalil, 2002] Khalil, H. (2002). Nonlinear Systems. Prentice Hall.

[Kiefer and Wolfowitz, 1952] Kiefer, J. and Wolfowitz, J. (1952). Stochastic estimation of the maximum of a regression function. The Annals of Mathematical Statistics, 23(3):462-466.

[Klein, 1978] Klein, V. (1978). Aircraft parameter estimation in frequency domain. AIAA Journal, (1344).

[Klein, 1989] Klein, V. (1989). Estimation of aircraft aerodynamic parameters from flight data. Progress in Aerospace Sciences, 26:1-77.

[Kosmatopoulos et al., 1995] Kosmatopoulos, E., Polycarpou, M., Christodoulou, M., and Ioannou, P. (1995). High-order neural network structures for identification of dynamical systems. Neural Networks, IEEE Transactions on, 6(2):422-431.

[Larson et al., 2006] Larson, J., Bruch, M., and Ebken, J. (2006). Autonomous navigation and obstacle avoidance for unmanned surface vehicles. In In Proceedings of SPIE Unmanned Systems Technology, 8, pages $17-18$, Orlando, FL. 
[Larson et al., 2007] Larson, J., Bruch, M., Halterman, R., Rogers, J., and Webster, R. (2007). Advances in autonomous obstacle avoidance for unmanned surface vehicles. In In Proceedings to AUVSI's Unmanned Systems, Washington, D. C.

[Lefeber et al., 2003] Lefeber, E., Pettersen, K., and Nijmeijer, H. (2003). Tracking control of an underactuated ship. IEEE Transactions on Control Systems Technology, 11(1):52-61.

[Levin and Narendra, 1993] Levin, A. and Narendra, K. (1993). Control of nonlinear dynamical systems using neural networks: controllability and stabilization. Neural Networks, IEEE Transactions on, 4(2):192206.

[Levin and Narendra, 1996] Levin, A. and Narendra, K. (1996). Control of nonlinear dynamical systems using neural networks. II. observability, identification, and control. Neural Networks, IEEE Transactions on, 7(1):30-42.

[Liberzon, 2003] Liberzon, D. (2003). Switching in Systems and Control. Birkhäuser.

[Lin and Wu, 1982] Lin, P. L. and Wu, Y. C. (1982). Identification of multi-input multi-output linear systems from frequency response data. Journal of Dynamic Systems, Measurement, and Control, 104(1):5864.

[Ljung, 1999] Ljung, L. (1999). System Identification: Theory for the User. Prentice Hall, 2 edition.

[Manley et al., 2000] Manley, J. E., Marsh, A., Cornforth, W., and Wiseman (2000). Evolution of the autonomous surface craft autocat. In IEEE Oceans, Providence, RI.

[Martins et al., 2007a] Martins, A., Almeida, J., Ferreira, H., Silva, H., Dias, N., Dias, A., Almeida, C., and Silva, E. (2007a). Autonomous surface vehicle docking manoeuvre with visual information. In IEEE International Conference on Robotics and Automation, pages 4994-4999.

[Martins et al., 2007b] Martins, A., Ferreira, H., Almeida, C., Silva, H., Almeida, J. M., and Silva, E. (2007b). ROAZ and ROAZ II autonomous surface vehicle design and implementation. In Proceedings of the International Lifesaving Congress, La Coruna, Spain.

[McKelvey, 1997] McKelvey, T. (1997). Frequency domain identification with instrumental variable based algorithm. In Proc. 16th Biennal Conference on Mechanical Vibaration and Noise, Sacramento, California. 
[McKelvey et al., 1996] McKelvey, T., Akçay, H., and Ljung, L. (1996). Subspace-based identification of infinite-dimensional multivariable systems from frequency-response data. Automatica, 32(6):885-902.

[Micaelli and Samson, 1993] Micaelli, A. and Samson, C. (1993). Trajectory tracking for unicycle-type and two-steering-wheels mobile robots. Technical Report 2097, INRIA, Sophia-Antipolis.

[Mišković et al., 2011] Mišković, N., Vukić, Z., Bibuli, M., Bruzzone, G., and Caccia, M. (2011). Fast in-field identification of unmanned marine vehicles. Journal of Field Robotics, 28(1):101-120.

[Morelli, 2000a] Morelli, E. A. (2000a). Identification of low order equivalent system models from flight test data. Technical report, NASA Langley Technical Report Server.

[Morelli, 2000b] Morelli, E. A. (2000b). Real-time parameter estimation in the frequency domain. Journal of Guidance Control and Dynamics, 23(5):812-818.

[Morelli and Klein, 2006] Morelli, E. A. and Klein, V. (2006). Aircraft System Identification: Theory and Practice. AIAA.

[Muske et al., 2008] Muske, K. R., Ashrafinon, H., Haas, G., McCloskey, R., and Flynn, T. (2008). Identification of a control oriented nonlinear dynamic USV model. In American Control Conference, Seattle, WA.

[Naeem et al., 2006a] Naeem, W., Xu, T., Chudley, J., and Sutton, R. (2006a). Design of an unmanned surface vehicle for environmental monitoring. In Proceedings of World Maritime Technology Conference, London, UK.

[Naeem et al., 2006b] Naeem, W., Xu, T., Sutton, R., and Chudley, J. (2006b). Design of an unmanned catamaran with pollutant tracking and surveying capabilities. In UKACC Control, 2006. Mini Symposia, pages 99-113.

[Naeem et al., 2008] Naeem, W., Xu, T., Sutton, R., and Tiano, A. (2008). The design of a navigation, guidance, and control system for an unmanned surface vehicle for environmental monitoring. Proceedings of the Institution of Mechanical Engineers, Part M: Journal of Engineering for the Maritime Environment, 222(2):67-79.

[Naeem W and GN, 2007] Naeem W, Sutton R, S. S. and GN, R. (2007). Experimental results on the development of a pollutant tracking unmanned surface vehicle. In Proceedings of IFAC Control Applications 
in Marine Systems Conference, Brac, Croatia.

[Narendra and Parthasarathy, 1990] Narendra, K. and Parthasarathy, K. (1990). Identification and control of dynamical systems using neural networks. Neural Networks, IEEE Transactions on, 1(1):4-27.

[Nguyen, 2008] Nguyen, H. D. (2008). Recursive identification of ship manoeuvring dynamics and hydrodynamics. In Mercer, G. N. and Roberts, A. J., editors, Proceedings of the 8th Biennial Engineering Mathematics and Applications Conference, EMAC-2007, volume 49 of ANZIAM J., pages 717-732.

[Nomoto et al., 1957] Nomoto, K., Taguchi, T., Honda, K., and Hirano, S. (1957). On the steering qualities of ships. Technical report, International Shipbuilding Progress, Vol. 4.

[Norrbin, 1970] Norrbin, N. H. (1970). Theory and observation on the use of a mathematical model for ship maneuvering in deep and confined waters. In 8th Symposium on Naval Hydrodynamics, Pasadena, California.

[Ødegård, 2009] Ødegård, V. (2009). Nonlinear identification of ship autopilot models. Master's thesis, Norwegian University of Science and Technology.

[Panteley et al., 1998] Panteley, E., Lefeber, A., Loria, A., and Nijmeijer, H. (1998). Exponential tracking control of a mobile car using a cascaded approach. In Proceedings of the IFAC Workshop on Motion Control, pages 221-226.

[Partington, 1992] Partington, J. R. (1992). Robust identification in $\mathcal{H}_{\infty}$. Journal of Mathematical Analysis and Applications, 166(2):428-441.

[Petrich, 2009] Petrich, J. (2009). Improved Guidance, Navigation, and Control for Autonomous Underwater Vehicles: Theory and Experiment. PhD thesis, Virginia Polytechnic Institute and State University, Blacksburg, Virginia.

[Petrich and Stilwell, 2011] Petrich, J. and Stilwell, D. J. (2011). Robust control for an autonomous underwater vehicle that suppresses pitch and yaw coupling. Ocean Engineering, 38:197-204.

[Pettersen and Nijmeijer, 1999] Pettersen, K. and Nijmeijer, H. (1999). Global practical stabilization and tracking for an underactuated ship-a combined averaging and backstepping approach. Modeling Identification and Control, 20(4):189-200.

[Petterson and Nijmeijer, 2001] Petterson, K. Y. and Nijmeijer, H. (2001). Underactuated ship tracking 
control; theory and experiments. International Journal of Control, 74(14):1435-1446.

[Rajesh and Bhattacharyya, 2008] Rajesh, G. and Bhattacharyya, S. (2008). System identification for nonlinear maneuvering of large tankers using artificial neural network. Applied Ocean Research, 30(4):256263.

[Rao and Unbehauen, 2006] Rao, G. and Unbehauen, H. (2006). Identification of continuous-time systems. Control Theory and Applications, IEE Proceedings -, 153(2):185-220.

[Robbins and Monro, 1951] Robbins, H. and Monro, S. (1951). A stochastic approximation method. The Annals of Mathematical Statistics, 22(3):400-407.

[Sakrison, 1967] Sakrison, D. J. (1967). The use of stochastic approximation to solve the system identification problem. IEEE Transactions on Automatic Control, 12(5).

[Sanathanan and Koerner, 1963] Sanathanan, C. and Koerner, J. (1963). Transfer function synthesis as a ratio of two complex polynomials. IEEE Transactions on Automatic Control, 8(1):56-58.

[Savitzky and Golay, 1964] Savitzky, A. and Golay, M. J. E. (1964). Smoothing and differentiation of data by simplified least squares procedures. Analytical Chemistry, 36(8):1627-1639.

[Selvam et al., 2005] Selvam, R. P., Bhattacharyya, S. K., and Haddara, M. (2005). A frequency domain system identification method for linear ship maneuvering. International Shipbuilding Progress, 52(1):527.

[Soetanto et al., 2003] Soetanto, D., Lapierre, L., and Pascoal, A. (2003). Adaptive, non-singular pathfollowing control of dynamic wheeled robots. In Decision and Control, 2003. Proceedings. 42nd IEEE Conference on, volume 2, pages 1765-1770.

[Son and Nomoto, 1981] Son, K. H. and Nomoto, K. (1981). On the coupled motion of steering and rolling of a high speed container ship. Journal of the Society of Naval Architecture, 150:232-244.

[Sonnenburg et al., 2010] Sonnenburg, C., Gadre, A., Horner, D., Kragelund, S., Stilwell, D. J., and Woolsey, C. A. (2010). Control-oriented planar motion modeling of unmanned surface vehicles. In OCEANS 2010, Seattle, WA.

[Sonnenburg and Woolsey, 2012] Sonnenburg, C. and Woolsey, C. A. (2012). An experimental comparison of two usv trajectory tracking control laws. In in Proceedings of MTS/IEEE Oceans, Hampton Roads, 
VA.

[Sonnenburg and Woolsey, 2013] Sonnenburg, C. and Woolsey, C. A. (2013). Modeling, identification, and control of an unmanned surface vehicle. Journal of Field Robotics. To appear.

[Sordalen and Canudas de Wit, 1993] Sordalen, O. and Canudas de Wit, C. (1993). Exponential control law for a mobile robot: extension to path following. Robotics and Automation, IEEE Transactions on, $9(6): 837-842$.

[Sri-Jayantha and Stengel, 1988] Sri-Jayantha, M. and Stengel, R. F. (1988). Determination of nonlinear aerodynamic coefficients using the estimation-before-modeling method. Journal of Aircraft, 25(9):796804.

[S.Sutulo and Soares, 2005] S.Sutulo and Soares, C. G. (2005). Simulation of a fast catamaran's manoeuvring motion based on a 6dof regression model. In Proceedings of International Conference on Fast Sea Transportation, St. Petersburg, Russia.

[Stalford, 1981] Stalford, H. L. (1981). High-alpha aerodynamic model identification of t-2c aircraft using the ebm method. Journal of Aircraft, 18(10):801-809.

[Stengel, 1986] Stengel, R. F. (1986). Optimal Control and Estimation. Dover Publications, Mineola, NY.

[Stilwell et al., 2011] Stilwell, D. J., Gadre, A. S., and Kurdila, A. J. (2011). A receding horizon approach to generating dynamically feasible plans for vehicles that operate over large areas. In Intelligent Robots and Systems (IROS), 2011 IEEE/RSJ International Conference on, pages 1140-1145.

[Stoica and Soderstrom, 1983] Stoica, P. and Soderstrom, T. (1983). Optimal instrumental-variable methods for identification of multivariable linear systems. Automatica, 19(4):425-429.

[Theodore et al., 2004] Theodore, C. R., Tischler, M. B., and Colbourne, J. D. (2004). Rapid frequencydomain modeling method for unmanned aerial vehicle flight control applications. Journal of Aircraft, 41(4):735-743.

[Thomasson and Woolsey, 2012] Thomasson, P. G. and Woolsey, C. A. (2012). Vehicle motion in currents. Journal of Oceanic Engineering.

[Thomsen et al., 2007] Thomsen, J. E., Guillory, V. G., and Benes, T. A. (2007). The Navy unmanned surface vehicle (USV) master plan. 
[T.Katayama et al., 2000] T.Katayama, Y.Ikeda, and H.Okumura (2000). A study on unstable motions of a planing craft in maneuvering-large amplitude motion due to periodic maneuvering motion. Journal of the Society of Naval Architects of Japan, 188:155-162.

[Toussaint et al., 2000] Toussaint, G. J., Basar, T., and Bullo, F. (2000). Tracking for nonlinear underactuated surface vessels with generalized forces. In IEEE Conference on Control Applications, Anchorage, Alaska.

[Wang and Zhang, 2001] Wang, Q.-G. and Zhang, Y. (2001). A novel FFT-based robust multivariable process identification method. Industrial Engineering Chemistry Research, 40(11):2485-2494.

[Wong and Polak, 1967] Wong, K. Y. and Polak, E. (1967). Identification of linear discrete time systems using the instrumental variable method. IEEE Transaction on Automatic Control, 12(6):707-718.

[Woolsey, 2011] Woolsey, C. A. (2011). Vehicle dynamics in currents. Technical Report VaCAS-2011-01, Virginia Center for Autonomous Systems, Blacksburg, VA.

[Xu et al., 2009a] Xu, B., Kurdila, A., and Stilwell, D. (2009a). A hybrid receding horizon control method for path planning in uncertain environments. In Intelligent Robots and Systems, 2009. IROS 2009. IEEE/RSJ International Conference on, pages 4887-4892.

[Xu et al., 2009b] Xu, B., Stilwell, D., and Kurdila, A. (2009b). Efficient computation of level sets for path planning. In Intelligent Robots and Systems, 2009. IROS 2009. IEEE/RSJ International Conference on, pages 4414-4419.

[Xu et al., 2010] Xu, B., Stilwell, D., and Kurdila, A. (2010). A receding horizon controller for motion planning in the presence of moving obstacles. In Robotics and Automation (ICRA), 2010 IEEE International Conference on, pages 974-980.

[Xu et al., 2012] Xu, B., Stilwell, D., and Kurdila, A. (2012). Fast path re-planning based on fast marching and level sets. Journal of Intelligent \& Robotic Systems, pages 1-15.

[Yoon and Rhee, 2003] Yoon, H. K. and Rhee, K. P. (2003). Identification of hydrodynamic coefficients in ship maneuvering equations of motion by estimation-before-modeling technique. Ocean Engineering, 30:2379-2404.

[Yoon et al., 2004] Yoon, H. K., Son, N. S., and Lee, C. M. (2004). Estimation of roll related hydrodynamic 
coefficients through the free running model tests. In OCEANS '04. MTTS/IEEE TECHNO-OCEAN '04, volume 2, pages 1086-1092.

[Young, 1970] Young, P. C. (1970). An instrumental variable method for real-time identification of a noisy process. Automatica, 6:271-287.

[Yu et al., 2008a] Yu, Z., Bao, X., and Nonami, K. (2008a). Course keeping control of an autonomous boat using low cost sensors. Journal of System Design and Dynamics, 2(1):389-400.

[Yu et al., 2008b] Yu, Z., Bao, X., and Nonami, K. (2008b). Course keeping control of an autonomous boat using low cost sensors. Journal of System Design and Dynamics, 2(1):389-400.

[Zimmermann and Mingori, 1996] Zimmermann, K. F. and Mingori, D. L. (1996). Identification of state space models using least squares or instrumental. In AIAA Guidance, Navigation, and Control Conference, San Diego, California. 


\title{
APPENDIX
}

\author{
A
}

\section{IDENTIFIABILITY CALCULATIONS}

An identifiability test, described in Grewal et. al [Grewal and Glover, 1976], is performed on all linear dynamic models shown in this dissertation. This test provides assurance that the identified parameters of each model uniquely define any given trajectory. Steady state behavior is constrained to mirror the identification procedure, though biasing terms are omitted to conform to the test's linearity restriction. It is trivial to note that the biasing terms do not affect the identifiability of these systems. 


\section{A.1 Nomoto's Model and Linearized Speed Model}

The Gramian for Nomoto's model and the linearized speed model is

$$
\boldsymbol{G}(\theta)=\left(\begin{array}{c}
\boldsymbol{D}(\theta) \\
\boldsymbol{C}(\theta) \boldsymbol{B}(\theta) \\
\boldsymbol{C}(\theta) \boldsymbol{A}(\theta) \boldsymbol{B}(\theta) \\
\vdots \\
\boldsymbol{C}(\theta) \boldsymbol{A}^{2 n-1}(\theta) \boldsymbol{B}(\theta)
\end{array}\right)=\left(\begin{array}{c}
\left(\begin{array}{ll}
1 & 0 \\
0 & 1
\end{array}\right)\left(\begin{array}{c}
0 \\
-k_{r} \theta_{1}
\end{array}\right) \\
\left(\begin{array}{ll}
1 & 0 \\
0 & 1
\end{array}\right)\left(\begin{array}{ll}
0 & 1 \\
0 & \theta_{1}
\end{array}\right)\left(\begin{array}{c}
0 \\
-k_{r} \theta_{1}
\end{array}\right) \\
\left(\begin{array}{ll}
1 & 0 \\
0 & 1
\end{array}\right)\left(\begin{array}{ll}
0 & 1 \\
0 & \theta_{1}
\end{array}\right)\left(\begin{array}{c}
0 \\
0 \\
-k_{r} \theta_{1}
\end{array}\right) \\
\left(\begin{array}{ll}
1 & 0 \\
0 & 1
\end{array}\right)\left(\begin{array}{ll}
0 & 1 \\
0 & \theta_{1}
\end{array}\right)^{3}\left(\begin{array}{c}
0 \\
0 \\
-k_{r} \theta_{1}
\end{array}\right)
\end{array}\right)=\left(\begin{array}{c}
k_{r} \theta_{1} \\
-k_{r} \theta_{1} \\
-k_{r} \theta_{1}^{2} \\
-k_{r} \theta_{1}^{2} \\
-k_{r} \theta_{1}^{3} \\
-k_{r} \theta_{1}^{3} \\
-k_{r} \theta_{1}^{4}
\end{array}\right)
$$

The Jacobian of the Gramian is

$$
\frac{\partial \boldsymbol{G}(\theta)}{\partial \theta}=\left(\begin{array}{c}
0 \\
-k_{r} \\
-k_{r} \\
-k_{r} \theta_{1} \\
-k_{r} \theta_{1} \\
-3 k_{r} \theta_{1}^{2} \\
-3 k_{r} \theta_{1}^{2} \\
-4 k_{r} \theta_{1}^{3}
\end{array}\right)
$$

which has rank 1, so Nomoto's model is identifiable. 


\section{A.2 Nomoto's Model with Sideslip}

The Gramian for Nomoto's model with sideslip is

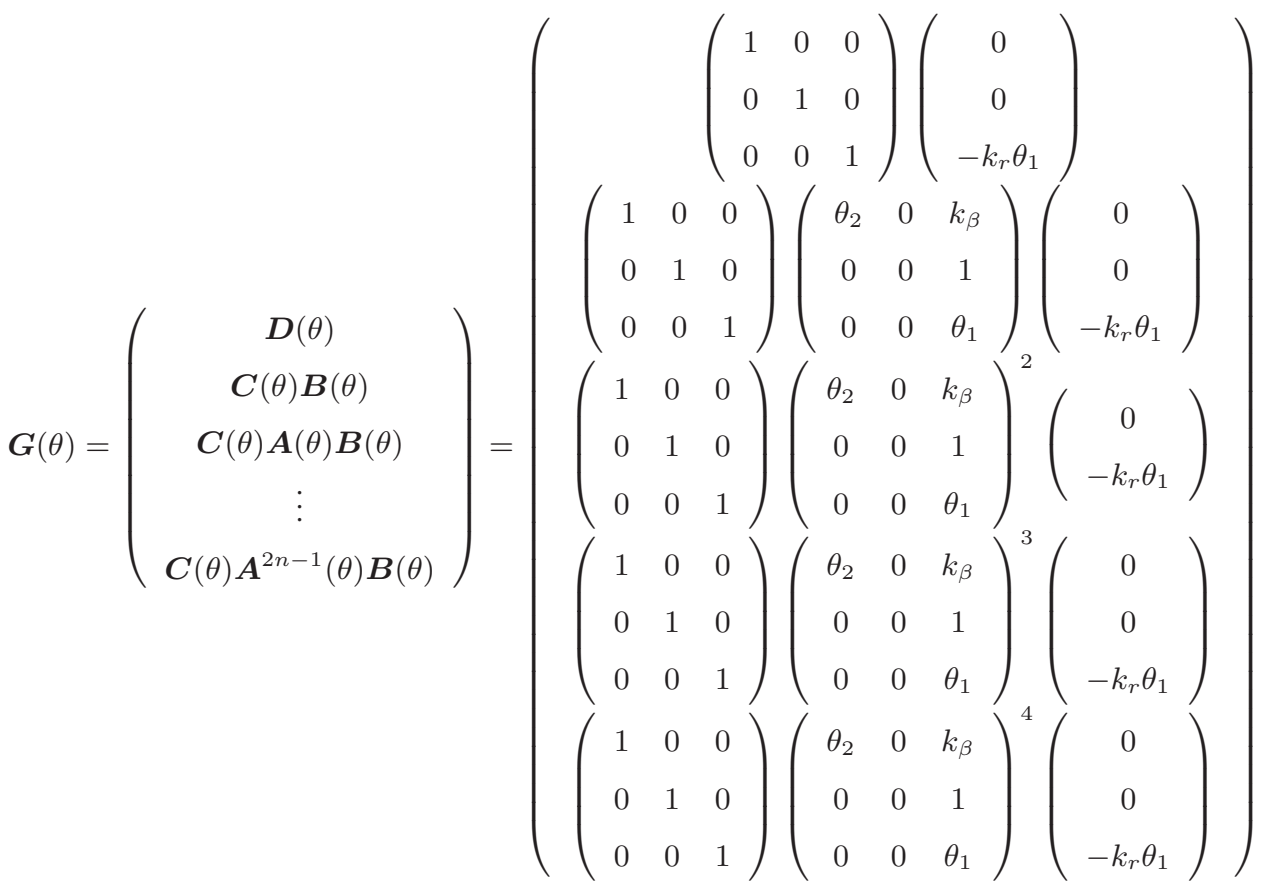

$$
\begin{aligned}
& \left(\begin{array}{c}
0 \\
0 \\
-k_{r} \theta_{1} \\
-k_{r} k_{\beta} \theta_{1} \theta_{2} \\
-k_{r} \theta_{1} \\
-k_{r} \theta_{1}^{2} \\
-k_{r} k_{\beta} \theta_{1} \theta_{2}\left(\theta_{1}+\theta_{2}\right) \\
-k_{r} \theta_{1}^{2} \\
-k_{r} \theta_{1}^{3} \\
-k_{r} k_{\beta} \theta_{1} \theta_{2}\left(\theta_{1}^{2}+\theta_{1} \theta_{2}+\theta_{2}^{2}\right) \\
-k_{r} \theta_{1}^{3} \\
-k_{r} \theta_{1}^{4} \\
\left.+\theta_{2} \theta_{1}^{2}+\theta_{2}^{2} \theta_{1}+\theta_{2}^{3}\right) \\
-k_{r} \theta_{1}^{4} \\
-k_{r} \theta_{5}
\end{array}\right)
\end{aligned}
$$


The Jacobian of the Gramian is

$$
\frac{\partial \boldsymbol{G}(\theta)}{\partial \theta}=\left(\begin{array}{cc}
0 & 0 \\
0 & 0 \\
-k_{r} & -k_{r} k_{\beta} \theta_{1} \\
-k_{r} k_{\beta} \theta_{2} & 0 \\
-k_{r} & 0 \\
-2 k_{r} \theta_{1} & -k_{r} k_{\beta} \theta_{1}\left(\theta_{1}+2 \theta_{2}\right) \\
-k_{r} k_{\beta} \theta_{2}\left(2 \theta_{1}+\theta_{2}\right) & 0 \\
-2 k_{r} \theta_{1} & 0 \\
-3 k_{r} \theta_{1}^{2} & -k_{r} k_{\beta} \theta_{1}\left(\theta_{1}^{2}+2 \theta_{2} \theta_{1}+3 \theta_{2}^{2}\right) \\
-k_{r} k_{\beta} \theta_{2}\left(3 \theta_{1}^{2}+2 \theta_{2} \theta_{1}+\theta_{2}^{2}\right) & 0 \\
-3 k_{r} \theta_{1}^{2} & 0 \\
-4 k_{r} \theta_{1}^{3} & -k_{r} k_{\beta} \theta_{1}\left(\theta_{1}^{3}+2 \theta_{2} \theta_{1}^{2}+3 \theta_{2}^{2} \theta_{1}+4 \theta_{2}^{3}\right) \\
\left.-4 \theta_{2} \theta_{1}^{2}+2 \theta_{2}^{2} \theta_{1}+\theta_{2}^{3}\right) & 0 \\
-5 k_{r} \theta_{1}^{4} & 0
\end{array}\right)
$$

which has rank 2, so Nomoto's model with sideslip is identifiable. 


\section{A.3 Planar Steering Model}

The Gramian for the planar steering model is

$$
\begin{aligned}
& \boldsymbol{G}(\theta)=\left(\begin{array}{c}
\boldsymbol{D}(\theta) \\
\boldsymbol{C}(\theta) \boldsymbol{B}(\theta) \\
\boldsymbol{C}(\theta) \boldsymbol{A}(\theta) \boldsymbol{B}(\theta) \\
\vdots \\
\boldsymbol{C}(\theta) \boldsymbol{A}^{2 n-1}(\theta) \boldsymbol{B}(\theta)
\end{array}\right) \\
& =\left(\begin{array}{c}
\left(\begin{array}{ll}
1 & 0 \\
0 & 1
\end{array}\right)\left(\begin{array}{c}
0 \\
-k_{r} \theta_{1}
\end{array}\right) \\
\left(\begin{array}{ll}
1 & 0 \\
0 & 1
\end{array}\right)\left(\begin{array}{ll}
0 & 1 \\
0 & \theta_{1}
\end{array}\right)\left(\begin{array}{c}
0 \\
-k_{r} \theta_{1}
\end{array}\right) \\
\left(\begin{array}{ll}
1 & 0 \\
0 & 1
\end{array}\right)\left(\begin{array}{ll}
0 & 1 \\
0 & \theta_{1}
\end{array}\right)\left(\begin{array}{c}
0 \\
-k_{r} \theta_{1}
\end{array}\right) \\
\left(\begin{array}{ll}
1 & 0 \\
0 & 1
\end{array}\right)\left(\begin{array}{ll}
0 & 1 \\
0 & \theta_{1}
\end{array}\right)
\end{array}\right) \\
& \left(\begin{array}{r}
-k_{v} \theta_{1} \\
-k_{r} \theta_{2}
\end{array}\right. \\
& =\left(\begin{array}{c}
-k_{v}\left(\theta_{1}^{2}+\theta_{2} \theta_{3}\right)-k_{r}\left(\theta_{1}+\theta_{4}\right) \\
-k_{v}\left(\theta_{1}+\theta_{4}\right) \theta_{3}-k_{r}\left(\theta_{4}^{2}+\theta_{2} \theta_{3}\right) \\
\left(\theta_{1}^{2}+\theta_{2} \theta_{3}\right)\left(-\left(\theta_{2} k_{r}+\theta_{1} k_{v}\right)\right)-\theta_{2}\left(\theta_{1}+\theta_{4}\right)\left(\theta_{4} k_{r}+\theta_{3} k_{v}\right)
\end{array}\right. \\
& \theta_{3}\left(\theta_{1}+\theta_{4}\right)\left(-\left(\theta_{2} k_{r}+\theta_{1} k_{v}\right)\right)-\left(\theta_{4}^{2}+\theta_{2} \theta_{3}\right)\left(\theta_{4} k_{r}+\theta_{3} k_{v}\right) \\
& \left(\theta_{1}^{3}+2 \theta_{2} \theta_{3} \theta_{1}+\theta_{2} \theta_{3} \theta_{4}\right)\left(-\left(\theta_{2} k_{r}+\theta_{1} k_{v}\right)\right)-\theta_{2}\left(\theta_{1}^{2}+\theta_{4} \theta_{1}+\theta_{4}^{2}+\theta_{2} \theta_{3}\right)\left(\theta_{4} k_{r}+\theta_{3} k_{v}\right) \\
& \left.\theta_{3}\left(\theta_{1}^{2}+\theta_{4} \theta_{1}+\theta_{4}^{2}+\theta_{2} \theta_{3}\right)\left(-\left(\theta_{2} k_{r}+\theta_{1} k_{v}\right)\right)-\left(\theta_{4}^{3}+2 \theta_{2} \theta_{3} \theta_{4}+\theta_{1} \theta_{2} \theta_{3}\right)\left(\theta_{4} k_{r}+\theta_{3} k_{v}\right)\right)
\end{aligned}
$$


The Jacobian of the Gramian has rank 4, so the linear planar steering model is identifiable. Specifically,

$$
\frac{\partial \boldsymbol{G}(\theta)}{\partial \theta}=\left(\begin{array}{c}
-k_{v} \\
0 \\
-2 k_{v} \theta_{1}-k_{r} \theta_{2} \\
-k_{v} \theta_{3} \\
-k_{v}\left(3 \theta_{1}^{2}+2 \theta_{2} \theta_{3}\right)-k_{r} \theta_{2}\left(2 \theta_{1}+\theta_{4}\right) \\
-\theta_{3}\left(k_{r} \theta_{2}+k_{v}\left(2 \theta_{1}+\theta_{4}\right)\right) \\
-k_{v} \theta_{3}\left(\theta_{1}^{2}+2 \theta_{4} \theta_{1}+3 \theta_{4}^{2}+2 \theta_{2} \theta_{3}\right)-2 k_{r}\left(2 \theta_{4}^{3}+3 \theta_{2} \theta_{3} \theta_{4}+\theta_{1} \theta_{2} \theta_{3}\right)
\end{array}\right)
$$

